\title{
In-situ Bruchversuche an \\ amorph/kristallinen Multilagen im \\ Transmissionselektronenmikroskop
}

\section{Dissertation}

zur Erlangung des mathematisch-naturwissenschaftlichen Doktorgrades

\author{
„Doctor rerum naturalium“ \\ der Georg-August-Universität Göttingen \\ im Promotionsprogramm ProPhys \\ der Georg-August University School of Science (GAUSS)
}

vorgelegt von

Andreas Kelling

geboren in

Korbach

Göttingen, 2015 


\section{Betreuungsausschuss}

Prof. C. A. Volkert

Institut für Materialphysik

Georg-August-Universität Göttingen

Prof. C. Ropers

IV. Physikalisches Institut - Halbleiterphysik

Georg-August-Universität Göttingen

\section{Mitglieder der Prüfungskommission}

Referentin:

Prof. C. A. Volkert

Institut für Materialphysik

Georg-August-Universität Göttingen

Korreferent:

Prof. M. Seibt

IV. Physikalisches Institut - Halbleiterphysik

Georg-August-Universität Göttingen

Weitere Mitglieder der Prüfungskommission:

Prof. H.-U. Krebs

Institut für Materialphysik

Georg-August-Universität Göttingen

Prof. C. Ropers

IV. Physikalisches Institut - Halbleiterphysik

Georg-August-Universität Göttingen

Prof. H. Hofsäss

II. Physikalisches Institut - Atom- und Kernphysik

Georg-August-Universität Göttingen

Prof. Vasily Moshnyaga

I. Physikalisches Institut

Georg-August-Universität Göttingen

Tag der mündlichen Prüfung: 17.11.2015 


\section{Inhaltsverzeichnis}

$\begin{array}{lll}1 & \text { Einleitung } & 1\end{array}$

2 Stand der Forschung und Grundlagen 5

2.1 Bruchmechanik . . . . . . . . . . . . . . . . . . . 5

2.1 .1 Bruchmechanik auf der Nanoskala . . . . . . . . . . . . . . . 10

2.2 Verformungsmechanismen in amorph/kristallinen Multilagenstrukturen

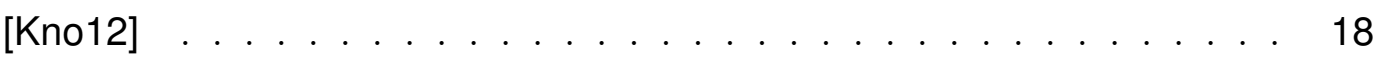

2.3 Stimulationen der Grenzflächen … . . . . . . . . . . . 23

2.3 .1 Adhäsionseffekte . . . . . . . . . . . . . . . 23

2.3 .2 Einfluss plastischer Energie auf Sprödbrüche . . . . . . . . . 27

3 Experimentelle Methoden 29

3.1 Verwendete Geräte . . . . . . . . . . . . . . . . . . . . . . . . . . . . . . 29

3.1 .1 lonenstrahlmikroskop . . . . . . . . . . . . . . . . . . . . 29

3.1 .2 Transmissionselektronenmikroskop. . . . . . . . . . . . . . . 30

3.1 .3 TEM-STM-Probenhalter. . . . . . . . . . . . . . . . . . . . . . 35

3.2 Proben . . . . . . . . . . . . . . . . . . . . . . . . . . 36

3.3 In-situ Bruchversuch $\ldots \ldots \ldots \ldots$

$3.3 .1 \quad$ Präparation der Geometrien $\ldots \ldots \ldots$

3.3 .2 Realisierung im TEM . . . . . . . . . . . . . . . . . . . . 39

3.4 Auswertung der Bruchversuche. . . . . . . . . . . . . . . . . . . . 42

3.4 .1 Energiefreisetzungsrate . . . . . . . . . . . . . . . . . 42

3.4 .2 Plastische Zone . . . . . . . . . . . . . . . . . . . . . . 45

3.5 FEM-Simulationen . . . . . . . . . . . . . . . . . . . 46

3.5 .1 COMSOL Multiphysics $\ldots \ldots \ldots$

4 Experimentelle Ergebnisse $\quad 49$

4.1 Charakterisierung der Multilagen . . . . . . . . . . . . . . . . . . . . 49

$4.1 .1 \quad$ EELS-Analyse der Grenzflächen . . . . . . . . . . . . . . . 53

4.2 FEM-Simulationen der Bruchgeometrie $\ldots \ldots \ldots \ldots$ 
4.3 Bruchversuche an Einzelschichten . . . . . . . . . . . . . . . . . 62

4.3 .1 Titan-Schicht . . . . . . . . . . . . . . . . . . . . . 62

4.3 .2 Zirkonoxid-Schicht . . . . . . . . . . . . . . . . . . . . . . . 67

4.4 Bruchversuche entlang der Grenzflächen $\ldots \ldots \ldots$

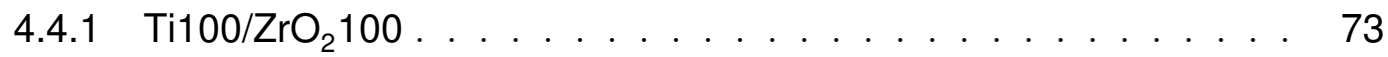

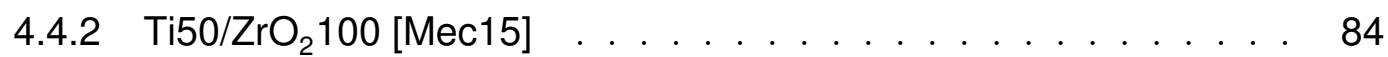

$4.4 .3 \mathrm{Ti}^{1} 0 / \mathrm{ZrO}_{2} 100 \ldots \ldots \ldots$

4.4 .4 Plastische Zone . . . . . . . . . . . . . . . . . . . . . . . . . . 97

4.4 .5 Energiefreisetzungsrate . . . . . . . . . . . . . . . . . 98 98

4.5 Bruchversuche senkrecht zu den Grenzflächen . . . . . . . . . . . . 100

$4.5 .1 \mathrm{Ti1}^{2} 00 / \mathrm{ZrO}_{2} 100 \ldots \ldots \ldots \ldots$

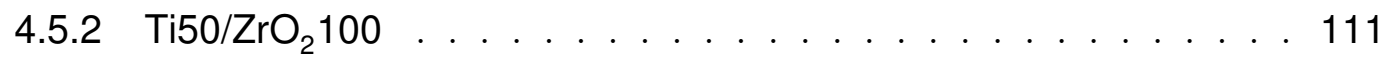

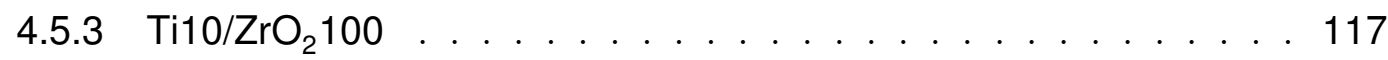

4.5 .4 Plastische Zone . . . . . . . . . . . . . . . . . . . . 125

4.5 .5 Energiefreisetzungsrate . . . . . . . . . . . . . . . . . 125

4.6 Einfluss einer Gasatmosphäre auf das Bruchverhalten . . . . . . . . . . 128

4.6 .1 Wasserstoff-Atmosphäre . . . . . . . . . . . . . . . 128

4.6 .2 Wasser-Atmosphäre . . . . . . . . . . . . . . . . . 133

4.6 .3 EELS Analyse in der Gasatmosphäre $\ldots \ldots$

$\begin{array}{lll}5 \text { Diskussion } & 137\end{array}$

5.1 Einzelschichten . . . . . . . . . . . . . . . . . . . . 138

5.1 .1 Titan-Einzelschichten . . . . . . . . . . . . . . . . . 138

5.1 .2 Zirkonoxid-Einzelschichten . . . . . . . . . . . . . . . 141

5.2 Bruchversuche der Multilagen $\ldots \ldots \ldots$

5.2 .1 Entlang der Grenzflächen . . . . . . . . . . . . . . . . . . . 142

5.2 .2 Senkrecht zu den Grenzflächen . . . . . . . . . . . . . 147

5.2 .3 Plastische Zone . . . . . . . . . . . . . . . . . . . . . . . . 150

$5.2 .4 \quad$ Bruchzähigkeiten und Energiefreisetzungsraten . . . . . . . . 153

5.3 Bruchverhalten in einer Gasatmosphäre . . . . . . . . . . . . . 158

6 Zusammenfassung 161

\begin{tabular}{|l|l|}
\hline Anhang A Bruchversuch im SEM & 165
\end{tabular}

\begin{tabular}{ll}
\hline Anhang B Zugversuche & 167
\end{tabular}

\begin{tabular}{|ll}
\hline Anhang C Kristallisation im FIB & 171
\end{tabular} 
Literaturverzeichnis

\begin{tabular}{ll}
\hline Abbildungsverzeichnis & 189
\end{tabular}

\begin{tabular}{ll}
\hline Abkürzungsverzeichnis & 193
\end{tabular} 


\section{Einleitung}

Das Versagen von Materialien oder Werkstücken ist ein Problem, mit dem sich Forscher und Ingenieure vom Beginn der Entwicklung neuer Materialien bis hin zu ihrer Anwendung beschäftigen müssen. Mit der zunehmenden Komplexität neuer Technologien steigen die Anforderungen an neue Materialien ununterbrochen und damit auch an deren Charakterisierungen. Besonders das Verständnis des Versagens der Materialien, das in den seltensten Fällen gewünscht wird, ist für die Weiterentwicklung der Technologien von essentieller Wichtigkeit.

Die Erkenntnisse auf dem Gebiet der Bruchmechanik haben bereits einen großen Beitrag zum Verständnis und auch zur Kontrolle des Bruchverhaltens verschiedenster Materialien und Geometrien geleistet. Jedoch werden durch die voranschreitende Miniaturisierung der Bauteile in nahezu allen Anwendungsgebieten und das damit veränderte Bruchverhalten der Materialien neue Modelle und Charakterisierungsmethoden notwendig, die im aktuellen Fokus der Forschung stehen (zum Beispiel Kum10,Jay15, Völ15]). Besonders für Nanokompositmaterialien, die aufgrund ihrer längenskalenabhängigen Eigenschaften und den vielen Grenzflächen interessant für neue Anwendungen sind, fehlt ein vollständiges, grundlegendes Verständnis der bruchmechanischen Konzepte und Parameter der Rissbildung und des Risswachstums. Die Forschung auf dem Gebiet der Bruchmechanik hat bereits hervorgebracht, dass für kleine Probendimensionen ein grundlegend verschiedenes Bruchverhalten beobachtet werden kann, als es die Erkenntnisse der konventionellen Bruchmechanik erwarten lassen Kum03, Wan03, You04, You05, Che05a, You06, Mos06a, Ovi07, Zha11. Dabei gibt es verschiedene Ansätze, um das veränderte Bruchverhalten auf der Nanoskala zu erklären. Für Silicium wird zum Beispiel angenommen, dass die Versetzungen in der Nähe einer Rissspitze dichter gepackt werden, wodurch eine höhere Bruchfestigkeit erklärt werden kann Bea11. Ein Mechanismus, der auch für verschiedenste Materialien anwendbar ist, fehlt jedoch bisher. Eine solche mechanistische Beschreibung des Bruchverhaltens in kleinen Probendimensionen wäre von großem Nutzen für die Entwicklung 
neuer, speziell zugeschnittener Materialien mit einem kontrollierbaren Bruchverhalten. Es ist bekannt, dass für duktile Materialien eine Verkleinerung der Probendimension zu einem Verlust der Bruchzähigkeit führt, wobei hingegen in spröden Materialien eine Verhärtung beobachtet wird Now07. Mit kleineren Probendimensionen steigt im Allgemeinen die Festigkeit in duktilen Materialien (zum Beispiel [Mor97,Uch04,Vol06, Uch09, Yu12]). Dieser Anstieg wirkt sich auf das Bruchverhalten aus, sodass mit höheren Festigkeiten höhere Bruchzähigkeiten zu erwarten sind und dementsprechend der Verringerung durch die Miniaturisierung entgegengesetzt wird [Kum03, Mey06, Koc07, Ovi07].

Aufgrund der Beobachtung einer längenskaligen Bruchzähigkeit und die damit verknüpfte Frage nach der Übertragbarkeit der tabellarisierten Werte Gra13] auf die Nanoskala ist es notwendig, die nanoskaligen Materialien auf der Längenskala zu untersuchen, in der sie letztlich auch zur Anwendung kommen (zum Beispiel Kan05, Kum11, Sch12a, Jay15, Vö115]). Als vielversprechende Methode haben sich hier die in-situ Experimente in einem Elektronenmikroskop herausgestellt [DM05, Kum10, Iqb12, Liu13]. Da in den kleineren Proben die Größe der plastischen Zone im Bereich der tatsächlichen Probendimensionen liegt, können für diese Bruchversuche nicht unbedingt die Bedingungen eines ebenen Dehnungszustandes oder die ASTM Standards AST13 erfüllt werden. Es ist daher notwendig, den Einfluss der plastischen Verformung auf das Rissverhalten zu berücksichtigen und neue Modelle zu entwickeln.

Weiterhin kann die Ausbildung eines Risses und damit das Versagen eines Materials unterhalb einer kritischen, charakteristischen Größe komplett unterdrückt werden How12 und die Materialien daher eine Festigkeit im Bereich der theoretischen Festigkeit eines perfekten Kristalls erreichen Gao03. Gerade in biologischen Materialien wie Knochen, Zähnen, Holz oder auch Schneckenhäusern KS96, Kam00] hat die Analyse gezeigt, dass sie oft bezüglich der mechanischen Eigenschaften und des Bruchverhaltens optimiert sind. Eine lamellare Struktur der Materialien und die Kombination verschiedenster Materialien resultieren oft in einem Mehrwert bezüglich der Eigenschaften des Komposits im Vergleich zu den einzelnen Komponenten Den05].

Die besonderen Eigenschaften funktionaler Kompositmaterialien haben diese in den Fokus der Forschung und der Industrie gebracht, da durch die geeignete Wahl der Materialien gezielt Komposite mit optimalen Eigenschaften hergestellt werden können. Solche, gezielt einstellbare Eigenschaften (chemisch, optisch, elektrisch usw.) sind für die verschiedensten Anwendungsgebiete von besonderem Interesse [Cho99, Par00, Voe01, Ver12.

In den meisten Fällen ist ein umfassendes Verständnis der besonderen Eigenschaften in den Kompositmaterialien jedoch nicht vorhanden. Auch im Bezug auf die mechanischen Eigenschaften fehlt noch dieses grundlegende Verständnis, sodass gerade diese die aktuelle Forschung prägen [Mis07, Liu12, Kno13]. Eine Kompositstruktur aus amorphen 
und kristallinen Komponenten zeigt eine Besonderheit hinsichtlich der mechanischen Eigenschaften des Komposits. So ist es möglich, dass zum Beispiel die Festigkeit der amorphen Komponente und die Duktilität der kristallinen Komponente in der Kompositstruktur erhalten bleiben. Dem hingegen können ungeeignete Eigenschaften für die Anwendung (zum Beispiel die geringe Duktilität der amorphen Komponenten) nahezu komplett unterdrückt werden. Unter Umständen ist sogar eine erhöhte Duktilität oder Festigkeit gegenüber den Einzelkomponenten möglich [Liu12,Wan07]. Im Allgemeinen werden die verbesserten mechanischen Eigenschaften über einen längenskalenabhängigen Verformungsmechanismus erklärt. In kristallinen Metallschichten steigt die Festigkeit mit kleinerer charakteristischer Größe [Mor97, Uch04,Vol06, Kra10] und weiterhin verspröden die Metallschichten, da die Versetzungsaktivität in kleinen Proben limitiert ist Mey06. In amorphen Materialien wird in kleinen Dimensionen die Unterdrückung der Scherbandbildung bei einer charakteristischen Größe von etwa $10 \mathrm{~nm}$ bis $100 \mathrm{~nm}$ beobachtet [Wan07].

Nicht nur die mechanischen Eigenschaften eines Komposits können das Bruchverhalten entscheidend beeinflussen, sondern auch die Einflüsse durch die Umgebung, in der die Komposite oder auch einkomponentige Werkstücke zum Einsatz kommen. Die zwei wichtigsten Effekte sind hier die Spannungsrisskorrosion und die Versprödung der Materialien in einer reaktiven Umgebung. Gerade in der Medizintechnik ist der Einfluss der Umgebung auf die Lebensdauer eines Implantates enorm wichtig, sodass ein tiefes Verständnis, wie eine reaktive Umgebung die Eigenschaften der Materialien und deren Bruchverhalten beeinflusst, unabdingbar ist.

Mit dem Verständnis dieser Effekte kann zudem eine Methodik entwickelt werden, mit der es möglich wird, Kompositmaterialien so durch eine Umgebung zu beeinflussen, dass der Risspfad gezielt gewählt werden kann. Die kontrollierte Auswahl des Risspfades ermöglicht somit ein effizientes Recycling von Kompositmaterialien. Die Kontrolle des Risspfades könnte hier über die gezielte Versprödung eines der Materialien oder die Schwächung einer der Grenzflächen erfolgen. Da sich der Riss im Allgemeinen entlang der schwächsten Richtung ausbreitet, wäre die Beeinflussung der Grenzflächen durch die Segregation eines geeigneten Materials eine vielversprechende Idee Komposite effizient zu recyclen. Weiterhin ist es vorstellbar Materialsysteme zu entwickeln, in denen die Adhäsion zwischen den Materialien gesteuert werden kann und so die Adhäsion von außen beeinflusst wird, sodass eine Trennung der Materialien an ihrer Grenzfläche möglich ist. Die Natur liefert hier ein exzellentes Beispiel anhand der Füße von Geckos, wie Adhäsion kontrolliert werden kann.

Im Rahmen dieser Dissertation wird ein in-situ Bruchversuch in Anlehnung an makroskopische Experimente entwickelt, der es erlaubt, auf der Nanometerskala kontrollierbare Bruchversuche in einem Transmissionselektronenmikroskop durchzuführen. Als Materialsystem wird eine amorph/kristalline Multilagenstruktur bestehend aus poly- 
kristallinen Titan-Schichten und Zirkonoxidschichten gewählt. Die Bruchversuche werden in zwei Geometrien realisiert, so kann das Risswachstum parallel und senkrecht zu den Grenzflächen untersucht werden. Durch die systematische Analyse des Bruchverhaltens in Abhängigkeit der Schichtdicke der kristallinen Komponente können zudem Einblicke in den Einfluss der Längenskala auf das Bruchverhalten gewonnen werden. Durch die Möglichkeit eine reaktive Gasatmosphäre in das Mikroskop einzubringen, werden zusätzlich Bruchversuche unter dem Einfluss einer Wasserstoff- und Wasserdampfatmosphäre durchgeführt. Die Veränderungen im Bruchverhalten der Multilagenstrukturen können auf geänderte, mechanische Eigenschaften der einzelnen Komponenten zurückgeführt werden, sodass damit eine mögliche Strategie zur Kontrolle des Risspfades demonstriert werden kann.

Die Dissertation gliedert sich in sechs Kapitel. Nachfolgend werden die physikalischen Grundlagen und der aktuelle Stand der Forschung auf dem Gebiet der Bruchmechanik mit dem Fokus der Bruchversuche auf der Nanoskala dargestellt. In Kapitel 3 werden die experimentellen Methoden beschrieben. Hier wird der entwickelte Bruchversuch und dessen Präparation und Auswertung ausführlich geschildert. In Kapitel 4 werden nacheinander die experimentellen Beobachtungen der Bruchversuche an den Einzelschichten, in der Geometrie entlang der Grenzflächen, in der Geometrie senkrecht zu den Grenzflächen und in einer Gasatmosphäre beschrieben. Des Weiteren werden die Ergebnisse der umfassenden Charakterisierung der Multilagen dargestellt. Die gewonnenen Erkenntnisse der Bruchversuche werden in Kapitel 5 im Vergleich mit der Literatur eingehend diskutiert. Es werden das Bruchverhalten in Abhängigkeit der Schichtdicke der kristallinen Schicht und das veränderte Bruchverhalten auf der Nanoskala erörtert. Der Einfluss einer Gasatmosphäre auf das Bruchverhalten wird abschließend zur Diskussion gestellt. Im sechsten Kapitel schließt diese Dissertation mit der Zusammenfassung der wesentlichen Erkenntnisse der in-situ Bruchversuche und einem Ausblick für weitergehende Experimente. 


\section{Stand der Forschung und Grundlagen}

Im folgenden Kapitel werden die wichtigsten theoretischen Grundlagen der Bruchmechanik dargestellt, die notwendig sind, um die experimentellen Ergebnisse einordnen und diskutieren zu können. Die grundlegenden Konzepte und Begrifflichkeiten der klassischen Bruchmechanik werden eingeführt.

Durch die voranschreitende Miniaturisierung in fast allen Bereichen der Forschung und der Entwicklung ergeben sich neue Herausforderungen bei der Realisierung von Bruchversuchen auf kleinen Längenskalen. Das möglicherweise veränderte Bruchverhalten bei kleinen Probendimensionen und die längenskalenabhängige Bruchzähigkeit, die als charakterisierende Materialeigenschaft angesehen wird, stehen im aktuellen Fokus der Forschung, deren Stand ebenfalls aufgezeigt wird.

\subsection{Bruchmechanik}

Das Versagen von Werkstücken in verschiedensten Anwendungen ist in den meisten Fällen unerwünscht, daher ist das Bruchverhalten und dessen Verständnis bei der Konzipierung und Realisierung neuer Werkstücke unerlässlich. Unabhängig davon, warum ein Material in seiner Anwendung versagt, kann dieses weitreichende Konsequenzen haben. Die Kontrolle über das Bruchverhalten kann daher als ultimatives Ziel der Forschung auf dem Gebiet der Bruchmechanik angesehen werden.

Es kann zwischen drei Brucharten unterschieden werden (vgl. Abbildung 2.1), wobei der Bruchmodus I für die Entwicklung neuer Materialien oder neuer Bauteile aufgrund des geringsten Widerstands gegenüber der Rissbildung und des Risswachstums am wichtigsten ist. Typischerweise führen Bruchversuche in Mode II- oder Mode IIIBruchgeometrie nicht zu einem Versagen der Probe. 


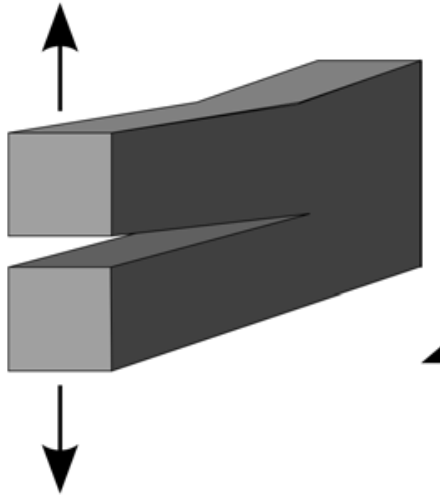

Mode I

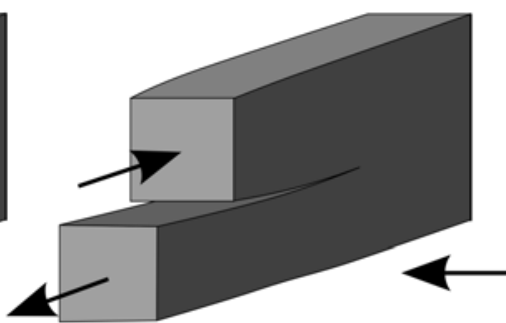

Mode II

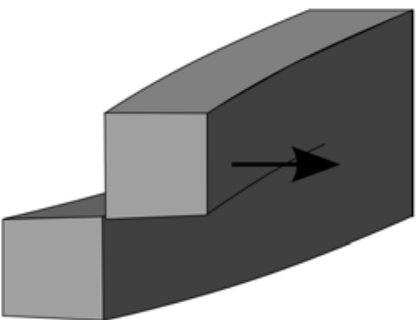

Mode III

Abbildung 2.1: Bruchmoden

Im Verlauf der Forschung im Bereich der Bruchmechanik haben sich zwei grundlegende Konzepte entwickelt, die es ermöglichen das Bruchverhalten zu beschreiben.

Griffith's Energieansatz besagt, dass ein Riss sich nur dann weiter ausbreitet, wenn die Energie, die zur Rissausbreitung zur Verfügung steht, größer ist als der Widerstand des Materials gegenüber dem Risswachstum Gri20]. In der Widerstandsfähigkeit des Materials sind alle dissipativen Prozesse während des Risswachstums enthalten. Die Weiterentwicklung dieses Ansatzes durch Irwin [Irw48 führt die Größe der Energiefreisetzungsrate $G$ ein. Diese beschreibt die Energie, die während des Risswachstums dissipiert wird bezogen auf die Oberfläche, die durch den Riss neu gebildet wird. Im Fall des Versagens eines Materials ist $G=G_{c}$ wobei $G_{c}$ die kritische Energiefreisetzungsrate ist, die somit als Maß für die Widerstandsfähigkeit bzw. Bruchzähigkeit des Materials fungiert. Im Vergleich zu der Festigkeit von Materialien hat $G_{c}$ eine ähnliche Bedeutung wie die Fließspannung $\sigma_{y s}$ eines Materials und $G$ kann mit der aufgebrachten Spannung $\sigma$ als Triebkraft der Verformung verglichen werden.

Der zweite Ansatz zur Beschreibung des Bruchverhaltens eines Materials beruht auf der Berechnung der Spannungskomponenten in allen Raumrichtungen an einem Punkt in der Nähe einer Rissspitze. Bei dieser Berechnung zeigt sich, dass alle Spannungskomponenten proportional jeweils zu einem einzelnen Faktor sind. Dieser Faktor wird als Spannungsintensitätsfaktor $K$ bezeichnet. Ist dieser Faktor $K$ bekannt, so ist damit auch der Spannungszustand in der Nähe der Rissspitze in einem linear-elastischen Material gegeben. Für bestimmte Werte von Dehnungen und Spannungen versagt das Material, sodass mit einem kritischen Spannungsintensitätsfaktor $K_{c}$ (auch als kritische Bruchzähigkeit bekannt) die Widerstandsfähigkeit des Materials beschrieben werden kann. Ein Versagen des Materials wird also erwartet, wenn $K=K_{c}$ gilt.

Der Spannungsintensitätsfaktor lässt sich wie folgt berechnen

$$
K=Y \sigma \sqrt{\pi a}
$$


wobei $Y$ eine dimensionslose Konstante ist, die den Einfluss der Geometrie sowie die Art der Verformung charakterisiert, $\sigma$ ist die aufgebrachte Spannung und $a$ die Risslänge. $Y=f(a / W)$ ist für viele Standardgeometrien bekannt und kann anhand der relevanten Dimensionen der Probe $W$ sowie der Risslänge $a$ berechnet werden [And95]. Der kritische Spannungsintensitätsfaktor $K_{c}$ ist für einen Mode I Riss typischerweise kleiner als die Faktoren der anderen beiden Moden $\left(K_{\text {IIc }}, K_{I I I c}>K_{I c}\right)$ And95.

Die Oberflächenenergie $\gamma$ der neuen Oberfläche, die durch einen Riss entstanden ist, kann aus der Bruchzähigkeit bzw. der kritischen Energiefreisetzungsrate berechnet werden. Für ein ideal sprödes Material gilt And69

$$
G_{c}=\frac{K_{C}^{2}}{E}=2 \gamma
$$

wobei $E$ der Elastizitätsmodul ist. Für duktile Materialien, in denen plastische Verformung stattfindet, bildet sich an der Spitze einer Kerbe eine plastische Zone, die mit steigender Belastung größer wird bis ein Riss gebildet wird. Innerhalb der plastischen Zone kann Energie dissipiert werden, sodass sich die Gleichung (2.2) zu

$$
G_{c}=2 \gamma+G_{p}
$$

verändert. Der dissipative Anteil $G_{p}$ enthält jede mögliche dissipative Kraft, wobei die plastische Verformung im Fall der duktilen Materialien den größten Anteil daran hat. Experimente wie auch Simulationen bestätigen, dass gerade bei Multilagenstrukturen mit Schichtdicken im Bereich von einigen Hundert Nanometern der dissipative Anteil $G_{p}$ der Energiefreisetzungsrate dominiert Dau98, Lan00. So kann eine starke Variation der makroskopisch gemessenen Bruchzähigkeit in Abhängigkeit der Schichtdicken der Multilagen beobachtet werden (vgl. Abschnitt 2.1.1).

Die plastische Zone in der Nähe der Spitze der Kerbe bzw. eines Risses beschreibt eine Zone, in der inelastische Verformung des Materials stattfindet. Die Größe dieser plastischen Zone $r_{p}$ kann über einen Ansatz eines Kräftegleichgewichts berechnet werden. Es gilt And69, Mei12

$$
r_{p} \approx \frac{1}{\pi}\left(\frac{K_{I Q}}{\sigma_{y s}}\right)^{2}
$$

wobei $\sigma_{y s}$ die Fließspannung des Materials ist.

Die Berechnung der Größe der plastischen Zone nach Gleichung (2.4) zeigt zum Beispiel nur bis zu Spannungen, die etwa der Hälfte der Fließspannung entsprechen, eine gute Übereinstimmung. Daraus folgt, dass für große plastische Zonen im Vergleich zu den Abmessungen der Proben die Anwendung der Konzepte der linear-elastischen Bruchmechanik nicht hinreichend genau sind. In den definierten Standards AST13] ist 
eindeutig für verschiedene Testgeometrien festgehalten, wie die Größe der plastischen Zone und die Dimensionen der Proben im Verhältnis zu einander stehen müssen, um aussagekräftige Ergebnisse zu erhalten.

Unter der Annahme eines linear-elastischen Materials können sowohl die Energiefreisetzungsrate $G$ als auch der Spannungsintensitätsfaktor $K$ zu der Beschreibung des Bruchverhaltens genutzt werden. Sie können wie folgt umgerechnet werden,

$$
G=\frac{K^{2}}{E^{\prime}}
$$

mit

$$
\begin{aligned}
& E^{\prime}=E \quad \text { für einen ebenen Spannungszustand } \\
& E^{\prime}=\frac{E}{1-\nu^{2}} \quad \text { für einen ebenen Dehnungszustand }
\end{aligned}
$$

wobei $\nu$ die Querkontraktionszahl des Materials ist.

Bereits in den ersten Untersuchungen des Bruchverhaltens von spröden Materialien zeigte sich eindeutig, dass es eine Diskrepanz zwischen den gemessenen Spannungen gibt, die nötig sind eine Probe zum Versagen zu bringen, und den theoretischen Spannungen, die notwendig sind, um atomare Bindungen aufzubrechen. Griffith entwickelte eine Theorie, die besagt, dass an mikroskopischen Schwachstellen des Materials die Spannungen lokal höher sind im Vergleich zur global aufgebrachten Spannung Ing13, Gri20, Irw48, And95. Neben den im Material enthaltenen Schwachstellen können auch maschinell hergestellte Kerben als absichtlich erzeugte Schwachstellen in einem Material dienen, an denen eine Spannungskonzentration während der Belastung aufgebaut wird.

Entscheidend für die Höhe der Spannung an der Spitze einer Kerbe ist deren Krümmungsradius [Kru04]. Es konnte in der Literatur gezeigt werden, dass die Spannungskonzentration an der Spitze einer Kerbe mit kleinem Krümmungsradius Werte annehmen kann, die bis zu zehn mal größer sind als die Fließspannung des Materials [Wei97]. Daher ist die lokale Spannungsverteilung an der Kerbe unter Umständen sehr verschieden zu der makroskopischen Fließspannung des Materials und kann somit das Bruchverhalten entscheidend beeinflussen. An Kerben mit größeren Krümmungsradien herrschen geringere Spannungen, sodass mehr extern aufgebrachte Spannung notwendig ist, um einen Riss zu bilden. Daraus resultiert die Möglichkeit, dass durch die höheren externen Spannungen ein spontanes Versagen wahrscheinlicher ist, da diese Spannungen höher als die für ein stabiles Risswachstum notwendigen Jay14.

Erstreckt sich die inelastische Verformung des Materials über einen größeren Bereich um die Rissspitze, sodass die linear-elastische Bruchmechanik nicht mehr anzuwenden ist, muss die Beschreibung des Bruchverhaltens über andere Konzepte erfolgen, die es ermöglichen das plastische Verhalten der Materialien zu erfassen. Die Beschreibung 


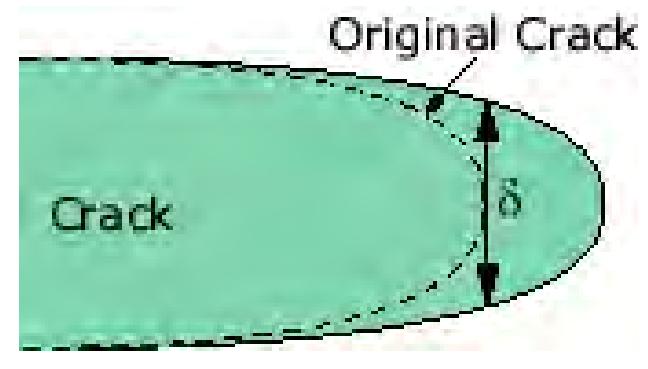

(a)

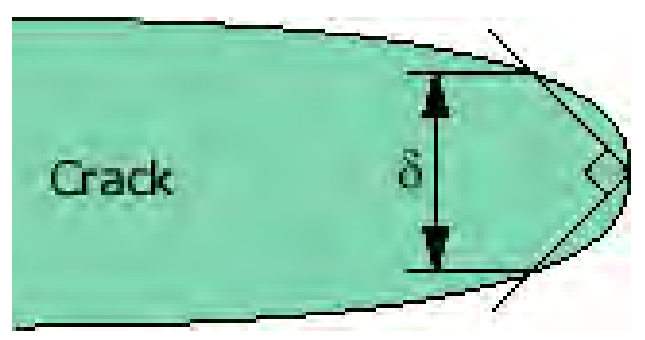

(b)

Abbildung 2.2: Definitionen CTOD aus http://www.efunda.com/formulae/solid_mechanics/ fracture_mechanics/fm_epfm_CTOD.cfm

erfolgt über Rissspitzenaufweitung (engl. crack tip opening displacement, CTOD) und das J-Konturintegral Ric68. Letzteres ist eine allgemeinere Version der Energiefreisetzungsrate, die im Falle eines linear-elastischen Materials identisch ist $(J=G)$ und die Energiefreisetzungsrate in einem nichtlinearen elastischen Körper mit einem Riss beschreibt.

Die Rissspitzenaufweitung kann auf zwei verschiedene Arten definiert werden (vgl. Abbildung 2.2, wobei die Definitionen äquivalent sind, wenn sich die Rissspitze zu einem Halbkreis abstumpft. Im Rahmen der Dissertation und in der Literatur wird bevorzugt die Definition aus Abbildung 2.2a genutzt. Sie bestimmt die CTOD im Verlauf des Risswachstums an der jeweiligen Position der Rissspitze.

Die Beschreibung des Bruchverhaltens über die CTOD beruht auf den experimentellen Beobachtungen von Wells Wel61, dass sich die Spitze einer Kerbe bzw. eines Risses bewegt, bevor es zum Versagen der Probe kommt. Durch die plastische Verformung in der Nähe der Rissspitze wird diese abgerundet. Die Abrundung ist proportional zur Festigkeit des Materials.

Die CTOD $\delta$ ist mit dem Spannungsintensitätsfaktor oder der Energiefreisetzungsrate direkt verknüpft, wenn die plastische Zone klein ist gegenüber den Abmessungen der Probe (small-scale yielding). Es gilt

$$
\begin{aligned}
\delta & =\frac{K_{I}^{2}}{m \sigma_{y s} E}=\frac{J_{I}}{m \sigma_{y s}} \\
\Leftrightarrow J_{I} & =\delta m \sigma_{y s}
\end{aligned}
$$

Der dimensionslose Parameter $m$ in den Gleichungen 2.6 und 2.7) berücksichtigt den kontinuumsmechanischen Zustand der Probe. So ist $m=1$, wenn ein ebener Spannungszustand (engl. plane stress) vorliegt, wohingegen $m=2$ für einen ebenen Dehnungszustand anzunehmen ist. 


\subsubsection{Bruchmechanik auf der Nanoskala}

Im nachfolgenden Abschnitt werden die grundlegenden Mechanismen und Konzepte beschrieben, die das Bruchverhalten bei der Verringerung der Längenskala entscheidend beeinflussen können. Diese Mechanismen dienen der abschließenden Diskussion und Einordnung der gewonnenen experimentellen Ergebnisse in Abschnitt 5

\section{Griffith's Kriterium}

Unabhängig von veränderten mikrostrukturellen oder mechanischen Eigenschaften nanoskaliger Materialien ist aufgrund der frühen Beobachtungen von Griffith ein längenskalenabhängiges Bruchverhalten zu erwarten. Das Kriterium für eine Rissausbreitung kann auf Basis der Änderung der Gesamtenergie des Systems bestimmt werden. Für eine unendlich große Platte (der Dicke $B$ ), die elastisch verformt (Spannung $\sigma$ ) wird und einen Riss der Länge $2 a$ enthält, verändert sich die Gesamtenergie wie folgt

$$
\Delta E \propto \frac{1}{2} \frac{\sigma^{2} a^{2} B}{E}-a B \gamma
$$

wobei der erste Term die Verringerung der potentiellen Energie und der zweite den Anstieg in der Oberflächenenergie aufgrund des Risses beschreibt. Anhand dieser Energiebalance kann die kritische Spannung zum Versagen des Materials berechnet werden. Diese Spannung skaliert mit der Länge $a\left(\sigma_{c} \propto 1 / \sqrt{a}\right)$.

\section{Unempfindlichkeit gegenüber Schwachstellen}

Die Proportionalität des kritischen Spannungsintensitätsfaktors mit der Spannung und der Risslänge aus Gleichung (2.1) zeigt einen weiteren Größeneffekt des Bruchverhaltens auf. Umso kleiner der Riss oder allgemeiner eine im Material enthaltene Schwachstelle ist (kleineres $a$ ), desto höher ist die Spannung, die notwendig ist, um den materialspezifischen kritischen Spannungsintensitätsfaktor $K_{c}$ zu erreichen. Daraus folgt, dass unterhalb einer kritischen Größe der Schwachstelle die notwendige Spannung zur Rissausbreitung die theoretische Festigkeit des Materials erreicht und daher das Material unempfindlich gegenüber enthaltenen Schwachstellen ist. Eine Schwachstelle, die größer als die kritische Größe ist, würde hingegen zum Versagen des Materials führen. Experimentell konnte nachgewiesen werden, dass in Aluminiumproben der Riss, der zum Versagen der Probe führt, nicht an der Spitze der Kerbe/Schwachstelle gebildet wird, sondern an zufälligen Positionen in der Probe. Diese Arbeit Kum09] unterstützt damit weitere Beobachtungen in der Literatur, die besagen, dass das Bruchverhalten von zum Beispiel biologischen Materialien (Knochen oder Zähne) unabhängig von den in ihnen enthaltenen Fehlstellen bzw. Hohlräumen ist [Gao03, Ji10]. 


\section{Längenskalenabhängige Festigkeit und Plastizität}

Die Verwendung nanokristalliner Materialien für verschiedenste Anwendungen ist bereits weit verbreitet, da sie aufgrund ihrer einzigartigen Eigenschaften („,smaller is stronger" Vol06, Deh06, Kra10, Gre11] oder des Hall-Petch-Verhaltens [Cho89, Car07]) neue Möglichkeiten eröffnen. Der Trend der ansteigenden Fließspannung mit kleinerer Probengröße wird auch für hexagonal dicht gepackte Materialien, wie es auch Titan ist, beobachtet YYu12]. Auch im Bereich der Bruchmechanik werden die nanoskaligen Materialien interessanter. In der Literatur wird beschrieben, dass sich die kritische Bruchzähigkeit mit kleinerer charakteristischer Größe der Materialien (Korngröße, Schichtdicke oder Probendimensionen) und der mechanischen Eigenschaften (Plastizität und Duktilität) verändert. Die längenskalenabhängige Bruchzähigkeit wird nachfolgend in Abschnitt 2.1.1 behandelt.

Die klassische Trennung in zwei verschiedene Bruchverhalten (duktil und spröde wie in Abbildung 2.3) ist für nanokristalline Materialien nicht mehr komplett aufrechtzuerhalten.

Experimentelle Arbeiten zeigen ein duktiles Bruchverhalten über die Bildung und das Wachstum von Hohlräumen in Materialien mit Korngrößen im Bereich zwischen $20 \mathrm{~nm}$ bis $100 \mathrm{~nm}$ Kum03, You04, You05, Che05a, You06, Ovi07, Kum11.

Nanokristallines Nickel mit einer vergleichbaren Korngröße von etwa $20 \mathrm{~nm}$ zeigt hingegen in Ermüdungsversuchen einen spröden Korngrenzenbruch Mos06a über die Bildung und das Wachstum von nanoskaligen Rissen an Tripelpunkten der Körner.

Entscheidend für den herrschenden Rissmechanismus in nanokristallinen Materialien ist der große Anteil von Korngrenzen im Verhältnis zum restlichen umgebenden Material. Zum einen dienen Korngrenzen als bevorzugte Nukleationspunkte von nanoskaligen Rissen, da die Bindung der Atome in den Korngrenzen schwächer ist als in den Körnern selbst. Zum anderen wird die Bildung von Hohlräumen und deren Wachstum an den Korngrenzen vereinfacht, da die Diffusion entlang der Korngrenzen im Vergleich zur Diffusion im Material deutlich erhöht ist.

In Abbildung 2.3 sind beide Rissmechanismen schematisch dargestellt. Unter dem Einfluss einer äußeren Spannung werden an den Korngrenzen nanoskalige Risse gebildet. Entscheidend für den Rissmechanismus ist, ob diese Risse zu Hohlräumen wachsen können (vgl. Abbildung 2.3a) oder als Spannungskonzentrationen zur Bildung weiterer Risse in der Umgebung (vgl. Abbildung 2.3b) dienen können. Welche der beiden Möglichkeiten eintritt, hängt von der Plastizität der jeweiligen Probe ab. Ist ein plastisches Fließen der Probe möglich, werden die Risse in Hohlräume umgewandelt (siehe Abbildung 2.3a). Die Hohlräume wachsen unter dem Einfluss der aufgebrachten Spannung soweit, dass sie miteinander in Kontakt kommen und immer größere Hohlräume bilden. Es kommt dadurch zu Einschnürungen und die Probe versagt über die Bildung und das 


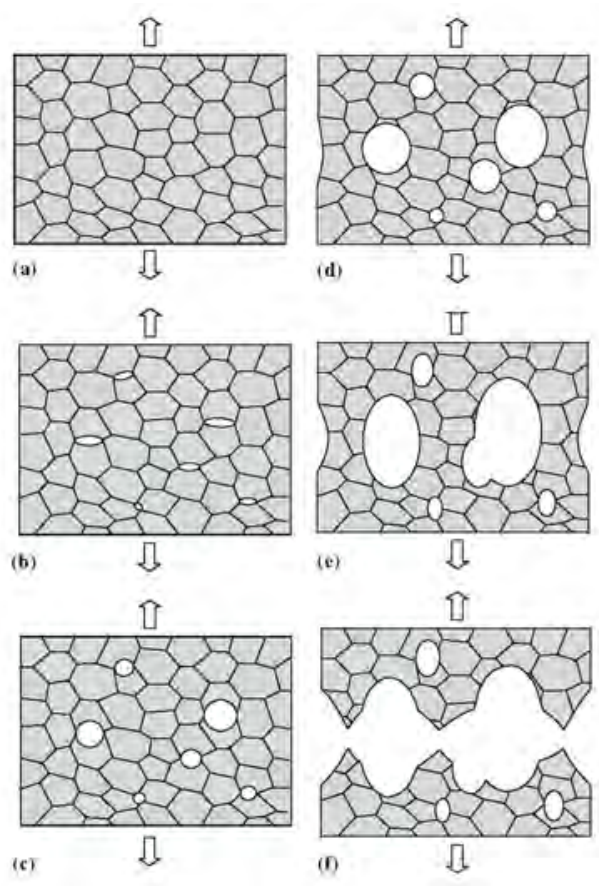

(a) duktil
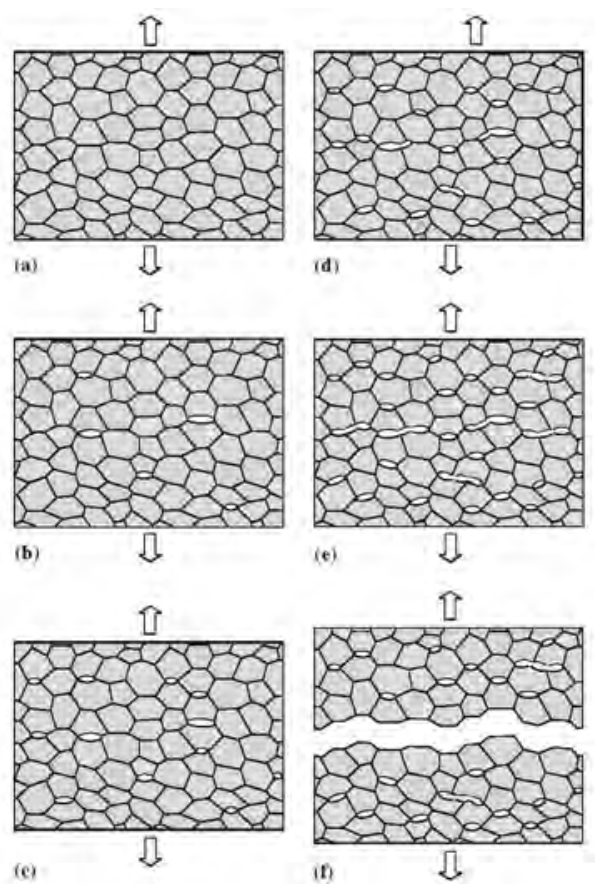

(b) spröde

Abbildung 2.3: Bruchmechanismen in nanokristallinen Materialien [Ovi07]

Zusammenwachsen von Hohlräumen, was einem duktilen Rissmechanismus entspricht. Kommt es jedoch zu keinem nennenswerten plastischen Fließen in der Probe, können in der Umgebung des Risses an den Korngrenzen mit steigender, aufgebrachter Spannung neue Risse gebildet werden und die Probe versagt katastrophal, was einem spröden Rissmechanismus gleichzusetzen ist (siehe Abbildung 2.3b.

Sowohl der spröde als auch der duktile Rissmechanismus stehen somit in direkter Konkurrenz und die Frage, ob nur der eine oder der andere oder beide Mechanismen gleichzeitig zu beobachten sind, hängt entscheidend von der Plastizität und auch von der Fließspannung, die die Größe der plastischen Zone bestimmt (vgl. Gleichung (2.4)), ab. Da die Fließspannung einem längenskaligen Verhalten unterliegt (zum Beispiel [Kum03, Vol06, Ovi07]), ist zu erwarten, dass die Größe der plastischen Zone und damit das Bruchverhalten ebenfalls mit einer charakteristischen Größe skalieren. Experimentell konnte gezeigt werden, dass es einen Übergang von duktilem zu sprödem Rissverhalten gibt, wenn die Korngröße bei nanokristallinen Nickel-Proben und Nickellegierungen von etwa $40 \mathrm{~nm}$ auf etwa $10 \mathrm{~nm}$ verringert wird [Li04,Li05]. Dieser Effekt ist bisher jedoch noch nicht vollständig nachzuweisen und dient lediglich als Anhaltspunkt für die Annahme, dass Materialien mit kleineren Körnern eine Tendenz zu eher sprödem Bruchverhalten aufweisen könnten.

Neben der Plastizität kann sich auch die Duktilität auf der Nanoskala verändern. Experimentell konnte gezeigt werden Zha11, dass mit geringer Schichtdicke die Duktilität eines auf größeren Skalen duktilen Materials in einer Multilagenstruktur aufgrund der 
längenskalenabhängigen Festigkeiten (smaller is stronger) und der Versetzungsaktivität verloren geht. Die verringerte Versetzungsaktivität steht in Verbindung mit der Wechselwirkung der bereits im Material enthaltenen Versetzungen mit den Grenzflächen. Diese werden an der Grenzfläche festgehalten und stauen sich gegen diese auf, sodass mehr Spannung notwendig ist, um neue Versetzungen zu nukleieren und das Material weiter plastisch zu verformen [Mis03, Mis07, Zhu08]. Für nanokristalline Materialien mit Korngrößen kleiner als etwa $30 \mathrm{~nm}$ wird beobachtet, dass eine Ansammlung von Versetzungen immer schwieriger wird und so immer weniger plastische Verformung erreicht werden kann Hsi94, Zhu04, Bud04, Kum10]. Eine fehlende Duktilität auf der Nanoskala verringert die Möglichkeit Verformungsenergie abzubauen und damit auch die Widerstandsfähigkeit des Materials.

\section{Abrundung der Rissspitze}

Eine geringe Plastizität auf der Nanoskala hat einen direkten Einfluss auf das Bruchverhalten. Aufgrund der fehlenden plastischen Verformung an der Rissspitze kann diese nicht vergleichbar stark abgerundet werden wie in einem Material, in dem ein plastisches Fließen möglich ist. Durch das Abrunden der Rissspitze wird Energie dissipiert, sodass das Material widerstandsfähiger gegenüber der Rissbildung wird.

\section{Rissspitzenabschirmung}

Weiterhin wird durch die veränderten plastischen Eigenschaften eines nanoskaligen Materials der Mechanismus der Rissspitzenabschirmung beeinflusst. Versetzungen, die von der Rissspitze aus nukleiert werden und sich von dieser entfernen, verringern durch ihr Spannungsfeld und die rückwirkenden Spannungen der Versetzungsquelle (hier die Rissspitze) den lokalen Spannungsintensitätsfaktor. Versetzungen in der Nähe der Rissspitze schirmen diese somit von dem sie umgebenden Spannungsfeld ab. Werden die Versetzungen aufgrund der Erhöhung der aufgebrachten Spannung von der Rissspitze weg bewegt, verringert sich die abschirmende Wirkung der Versetzungen und weitere Versetzungen können an der Rissspitze nukleiert werden.

\section{Einschränkung der plastischen Zone}

Bei Bruchversuchen auf der Nanoskala ist die Größe der plastischen Zone entscheidend für die Wahl der Abmessungen der Probe, um die Randbedingungen eines ebenen Dehnungszustandes zu erfüllen, damit quantitativ belastbare Daten gewonnen werden können. Die Größe der plastischen Zone ist auf der Nanoskala eingeschränkt, wenn sie die charakteristischen Längen, wie zum Beispiel die Schichtdicke, in einer Multilagenstruktur überschreitet. Daraus folgt, dass das Volumen, das plastisch verformt werden 
und somit zur Dissipation der Verformungsenergie beitragen kann, wesentlich kleiner und das Material weniger widerstandsfähig ist im Vergleich zu massiven Proben.

Ist die Schichtdicke einer duktilen Komponente vor der Rissspitze kleiner als die plastische Zone, die sich aufgrund der Belastung aufbauen würde, muss die plastische Zone an die Gegebenheiten (Form und Größe) angepasst werden. Da eine hohe Bruchzähigkeit im Wesentlichen durch eine plastische Verformung erreicht wird (vgl. Gleichung (2.3)), beeinflusst diese Einschränkung der plastischen Zone die Bruchzähigkeit Was96, Zha11.

Für Silicium-Einkristalle konnte in der Literatur gezeigt werden, dass bei einer geeigneten Wahl der Bruchgeometrie sowie der Größe der Proben relativ zur plastischen Zone ( $\leq 250 \mathrm{~nm}$ ), quantitativ vertrauenswürdige Werte der kritischen Bruchzähigkeit gemessen werden können [DM05, Deh06, Kra10, Jay15].

In-situ TEM-Dehnungsversuche an einkristallinen NiAl-Legierungen zeigen ein Bruchverhalten in Abhängigkeit von der Belastungsrichtung der Proben relativ zu den kristallographischen Richtungen Bai99]. So kann zum einen die Nukleation von Versetzungen an der Rissspitze und deren Bewegung durch den Kristall beobachtet werden, zum anderen wird ein sprödes Bruchverhalten ohne Versetzungsaktivität oder plastische Verformung während der Rissbildung beobachtet. Wenn das Rissverhalten mit einer Versetzungsaktivität einher geht, wird weiterhin festgestellt, dass sich die Rissausbreitungsrichtung in Bereichen hoher Versetzungsdichte verändert. Außerdem verlangsamt sich das Risswachstum in den Bereichen hoher Versetzungsdichte.

Das Bruchverhalten nanoskaliger Keramiken wird abschließend in diesem Abschnitt beschrieben Ovi15. Aufgrund der typischen NichtGleichgewichtsherstellungsbedingungen der Keramiken enthalten diese viele Hohlräume oder andere Fehlstellen, die sich bevorzugt an den Korngrenzen sammeln. Im Allgemeinen versagen Keramiken, wenn sie bei Raumtemperatur beansprucht werden, spröde. Das spröde Bruchverhalten begründet sich maßgeblich durch das Fehlen einer möglichen Versetzungsaktivität bei Raumtemperatur. Wie auch bereits für die nanokristallinen Metalle spielen die Korngrenzen eine entscheidende Rolle während des Bruchs der Keramiken. So können auch in Keramiken Korngrenzen als bevorzugte Nukleationspunkte von Rissen dienen. Korngrenzen sind besonders geeignet, um dort einen Riss zu bilden und entlang der Korngrenzen (intergranular) wachsen zu lassen, im Gegensatz zu einem Riss innerhalb eines Korns (intragranular). Da die Korngrenzen jedoch nur kurz und gekrümmt sind, muss auch der Riss gekrümmt entlang der Korngrenzen laufen, was einen intergranularen Bruchmechanismus erschwert.

$\mathrm{Ob}$ sich in einem nanokristallinen, keramischen Material ein Riss entlang der Korngrenzen oder durch die Körner hindurch ausbildet, entscheidet sich anhand materialspezifischer Eigenschaften und auch der Geometrie der Beanspruchung. 
Experimentell können sowohl intergranulares Bruchverhalten als auch die Rissbildung in Körnern (intragranular) beobachtet werden And09.

Durch die Verringerung der Korngröße ist auch in Keramiken ein Anstieg in der Festigkeit der Materialien in Abhängigkeit der Korngröße zu beobachten. Es werden Festigkeiten gemessen, die bis zu fünfmal höher sind im Vergleich zu grobkörnigen Keramiken (vgl. zum Beispiel [Kun04, Muk07]). Obwohl Keramiken weiterhin bei Raumtemperatur spröde versagen, ist eine verbesserte Bruchzähigkeit der nanokristallinen Keramiken zu beobachten. Die verbesserte Bruchzähigkeit steht in Verbindung mit einem Mechanismus, bei dem die lokalen Spannungen in der Nähe der Rissspitze durch lokale plastische Verformungen abgebaut werden können Szl05.

\section{Längenskalenabhängige Bruchzähigkeit}

Die Abhängigkeit der Bruchzähigkeit von der Längenskala wird maßgeblich durch die Veränderungen in dem Spannungszustand bestimmt, wenn die Probendimensionen verkleinert werden. Anhand experimenteller Modelle And69 kann eine kritische Größe $t_{1}$ beschrieben werden, unterhalb der nur die Bruchzähigkeit im ebenen Spannungszustand $K_{I C}^{\text {plane-stress }}$ gemessen werden kann. Überschreiten die Probendimensionen die kritische Größe $t_{2}$ können $K_{I C}$ gemessen werden, die konform zu den ASTM-Standards und damit vergleichbar mit anderen Daten in der Literatur (Ashby-Diagramme) sind (vgl. Abbildung 2.4).

Der Übergang von der Bruchzähigkeit im ebenen Dehnungszustand hin zum ebenen Spannungszustand bei kleineren Probendimensionen kann nicht genau beschrieben werden. In der Literatur wird von einer Näherung berichtet, die das Verhältnis der beiden Bruchzähigkeiten in Verbindung mit materialspezifischen Eigenschaften bringt Bro73. Das Verhältnis berechnet sich wie in Gleichung (2.9) angegeben über

$$
\frac{K_{I C}^{\text {plane-stress }}}{K_{I C}}=\sqrt{1+\frac{\varepsilon_{f} E}{12 \sigma_{y s}}}
$$

wobei $\varepsilon_{f}$ die Bruchdehnung und $\sigma_{y s}$ die Fließspannung des Materials sind.

Für NiAl-Einkristalle kann mit Gleichung 2.9 eine Abweichung von weniger als $10 \%$ der gemessenen Bruchzähigkeiten in kleinen Probenvolumina im Vergleich zu massiven Proben berechnet werden [qb12], sodass eine Veränderung im Spannungszustand durch die Miniaturisierung der Proben nur einen kleineren Einfluss hat. Diese Erwartung kann mit den gezeigten experimentellen Daten Iqb12 unterstützt werden. Für verschiedene kristallographische Orientierungen können die makroskopischen Bruchzähigkeiten gut reproduziert werden. Ein Größeneffekt der Bruchzähigkeit wird hier nicht beobachtet. Aufgrund der gewählten Probendimensionen in Iqb12 befinden sich die Proben in einem Übergangsbereich zwischen einem ebenen Dehnungszustand 


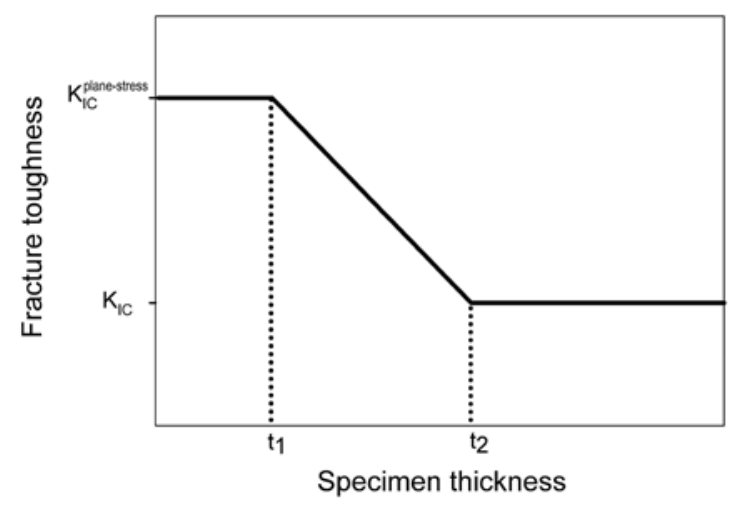

Abbildung 2.4: Anderson Modell aus [Mer11]

und einem ebenen Spannungszustand, sodass noch kein ausgeprägter Größeneffekt zu erwarten ist.

Auch in Ausbeulexperimenten (bulge tests) an amorphen Siliciumnitrid-Dünnschichten kann keine Veränderung der kritischen Bruchzähigkeit in Abhängigkeit der Schichtdicke gefunden werden Mer11]. Die Probendimensionen der Siliciumnitridschichten (20 nm bis $108 \mathrm{~nm})$ befinden sich in einem Übergangsbereich zwischen ebenen Dehnungszustand und ebenen Spannungszustand. Die erhaltenen Bruchzähigkeiten sind in dem angegebenen Schichtdickenbereich konstant und stimmen gut mit Literaturdaten von massiven Proben überein. Der Unterschied zwischen den Bruchzähigkeiten nach Gleichung (2.9) beträgt für das untersuchte Siliciumnitrid nur $2 \%$, sodass aufgrund der theoretischen Voraussagen kein Größeneffekt zu erwarten ist, was durch die Experimente bestätigt wird. Weiterhin schließen die Autoren in [Mer11] aus ihren Daten, dass für sehr spröde Materialien, die ein geringes Verhältnis von Elastizitätsmodul und Fließspannung aufweisen, im Allgemeinen zu erwarten ist, dass diese Materialien keinen Größeneffekt zeigen.

Anhand einer theoretischen Berechnung der Bruchzähigkeiten verschiedener Probengeometrien kann gezeigt werden, dass die Bruchzähigkeiten von Nanostrukturen invers proportional zur Quadratwurzel der charakteristischen Probendimension ist [Bea11]. Im Fall von spröden Materialien gilt so neben „smaller is stronger" auch „smaller is tougher".

Ausgehend von dem Konzept der Arbeit, die aufgebracht werden muss, um das Volumen um die Rissfläche herum zu verformen, kann für die verschiedenen Probengeometrien (dünne Schicht, Nanospheren und Säulen) die Abhängigkeit der Bruchzähigkeit von der Probendimension theoretisch berechnet werden (siehe Bea11). Experimentell kann gezeigt werden, dass diese Abhängigkeiten für dünne Kupfer-Schichten [Vol02] und auch für Nanospheren und Säulen [Moo07, Ger09, Öst09, Öst11, Bea11, How12] zutreffen.

Unter der Annahme, dass Versetzungen in der Nähe der Rissspitze diese abschirmen, 


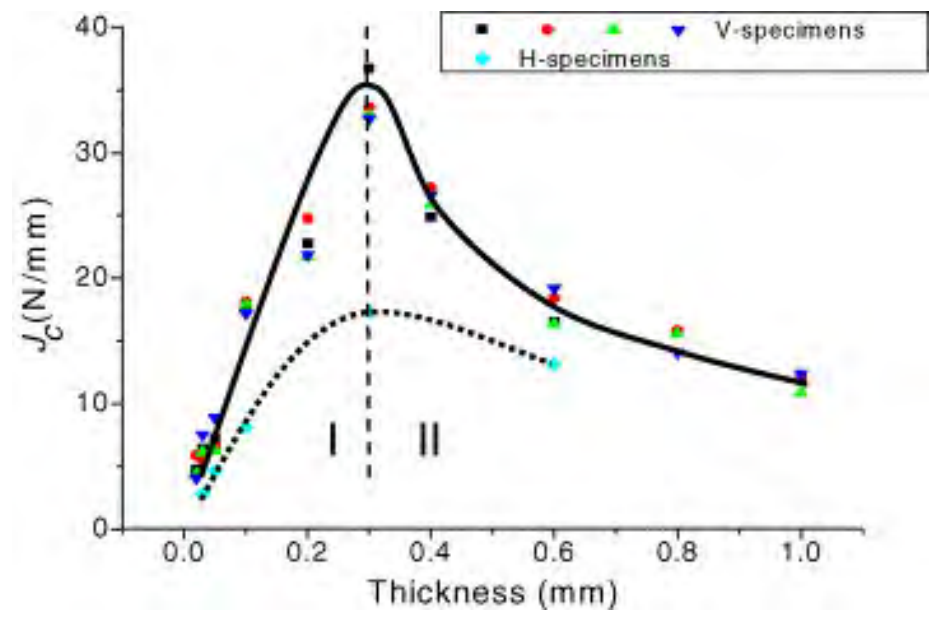

Abbildung 2.5: Gemessene kritische Energiefreisetzungsrate Kupferfolien mit Dicken zwischen $0,02 \mathrm{~mm}$ bis $1 \mathrm{~mm}$ aus [Kan05]

kann gezeigt werden, dass die Abhängigkeit von der kritischen Dimension konsistent ist mit einem versetzungsbasierten Versagensmechanismus. Dieser Mechanismus kann aufgrund fehlender experimenteller und auch theoretischer Daten nicht völlig bewiesen werden, jedoch bleibt rein experimentell festzustellen, dass kleine Proben nicht nur höhere Fließspannungen sondern auch erhöhte Bruchzähigkeiten zeigen („smaller is tougher") Bea11.

Die kritische Energiefreisetzungsrate bei der Rissbildung dünner Kupferfolien mit Schichtdicken zwischen $0,02 \mathrm{~mm}$ bis $1 \mathrm{~mm}$ zeigt ein klar schichtdickenabhängiges Verhalten wie es in Abbildung 2.5 dargestellt ist. In den Proben mit der geringsten Schichtdicke ermöglichen vorhandene Defekte ein Abscheren der Probe, bevor es zu einem signifikanten Gleiten von Versetzungen kommen kann, da die plastische Verformbarkeit bei diesen Schichtdicken gering ist. Der Versagensmechanismus ist somit eher spröde. Die Defekte oder Inhomogenitäten in der Mikrostruktur des Kupfers können bei geringen Schichtdicken hohe Spannungskonzentrationen in dem Material erzeugen, so dass die Widerstandsfähigkeit gegenüber Rissbildung und -wachstum stark geschwächt werden kann [Wan03]. Der abweichende Verlauf der Bruchzähigkeit bei kleinen Probendimensionen im Vergleich zu Abbildung 2.4 erklärt sich durch die fehlende Berücksichtigung der mikrostrukturellen Einflüsse in dem theoretischen Modell [And69].

Mit steigender Schichtdicke (I in Abbildung 2.5) erhöht sich die Verformbarkeit des Materials und die Defekte im Material spielen eine untergeordnete Rolle, so dass es $\mathrm{zu}$ einem duktilen Versagensmechanismus über die Bildung von Hohlräumen kommt und die Energiefreisetzungsrate bzw. die Bruchzähigkeit ansteigt. Im zweiten Bereich bei Schichtdicken größer als 0,3 mm sinkt die Bruchzähigkeit wieder, da zum einen die Verformbarkeit des Materials weiter ansteigt und zum anderen wird durch den Verlust 
der Randbedingungen (durch die geringen Schichtdicken) ein triaxialer Spannungszustand erzeugt, durch den Hohlräume einfacher gebildet werden können Kan05.

Untersuchungen an $500 \mathrm{~nm}$-dünnen Platin-Schichten (kollumnare Körner mit $20 \mathrm{~nm}$ Durchmesser) liefern eine um etwa eine Größenordnung kleinere Bruchzähigkeit (etwa $4 \mathrm{MPa} \mathrm{m}^{1 / 2}$ ) als Schichten mit Schichtdicken von mehreren Mikrometern [Mei12]. Die Analyse des Rissverhaltens liefert einen transgranularen Rissmechanismus (keine Versprödung der Korngrenzen durch Fremdatome) in Verbindung mit versetzungsbasierter Plastizität. Die kleinere Bruchzähigkeit in den dünnen Schichten ergibt sich durch den Übergang von einem ebenen Dehnungszustand hin zu einem ebenen Spannungszustand wie es auch in den Kupferfilmen in Wan03 beobachtet wird.

Freistehende Aluminium-Schichten mit einer Schichtdicke von etwa $100 \mathrm{~nm}$ bis $125 \mathrm{~nm}$ und einer mittleren Korngröße von $50 \mathrm{~nm}$ zeigen in in-situ Bruchversuchen in einem TEM kritische Bruchzähigkeiten im Bereich von $0,7 \mathrm{MPam}^{1 / 2}$ bis $1,1 \mathrm{MPam}^{1 / 2}$, die damit deutlich geringer sind als die Literaturwerte für Aluminium Kum09]. Die wesentlich geringere Bruchzähigkeit erklärt sich zum einen wiederum durch die Probendimensionen und dem damit verbundenen ebenen Spannungszustand und zum anderen durch die Mikrostruktur der Schichten. Da die Korngröße der Al-Schichten (mittlere Korngröße etwa $50 \mathrm{~nm}$ ) geringer ist als der minimale theoretische Abstand zwischen zwei Versetzungen von etwa $60 \mathrm{~nm}$ wird wenig Versetzungsaktivität beobachtet, die zu der geringen Bruchzähigkeit beiträgt.

In $\mathrm{Al}_{2} \mathrm{O}_{3} / \mathrm{Al} / \mathrm{Al}_{2} \mathrm{O}_{3}$-Schichtsystemen mit einer Metallschichtdicke zwischen $5 \mu \mathrm{m}$ bis $100 \mu \mathrm{m}$ wird ein Anstieg der Bruchzähigkeit mit der steigenden Schichtdicke gefunden Kru04. Der Anstieg steht in Verbindung mit der größeren Abrundung der Rissspitzen sowie einer ausgeprägteren plastischen Verformung in der Nähe der Rissspitze in dickeren Metallschichtdicken. Diese und weitere Beispiele aus der Literatur Dal89, Rei91, Gra92, McN96] zeigen, dass die Bruchzähigkeit in vielen Fällen nicht als materialspezifische Konstante angesehen werden kann und die Auswahl der Materialien für eine Anwendung anhand der Bruchzähigkeiten, die in massiven Proben gemessen werden, kritisch betrachtet werden muss.

\subsection{Verformungsmechanismen in amorph/kristallinen Multilagenstrukturen [Kno12]}

Zu Beginn der Diskussion des Bruchverhaltens der Multilagen werden die in der Literatur berichteten Verformungsmechanismen und die mechanischen Eigenschaften von Kompositmaterialien diskutiert. Da die $\mathrm{Ti} / \mathrm{ZrO}_{2}$-Multilagen, die in dieser Arbeit untersucht werden, bereits eingehend mechanisch charakterisiert worden sind Kno12], wird an dieser Stelle lediglich eine zusammenfassende Darstellung der Mechanismen gege- 
(a) freistehende Metallschicht

$$
\text { Metall }
$$

(b) Metallschicht auf Substrat

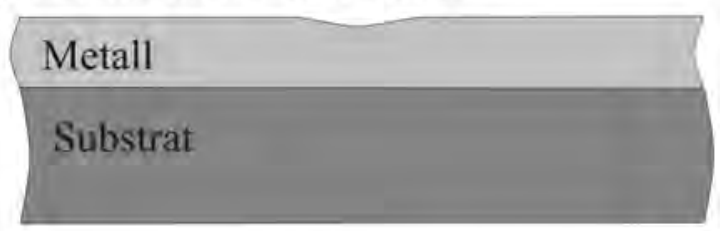

(c) Metallschicht auf Substrat, Delamination

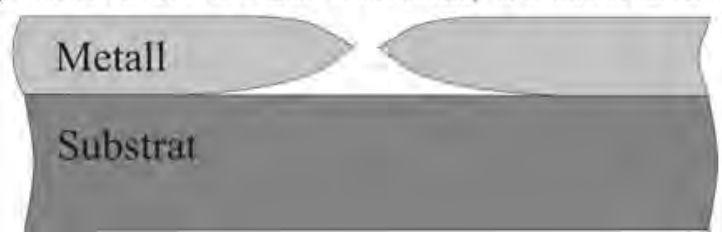

Abbildung 2.6: Verformungsmechanismen einer Metallschicht unter verschiedenen Randbedingungen aus [Kno12]

ben. Für detailliertere Beschreibungen sei an die entsprechende Literatur in $\overline{K n o 12}$ verwiesen.

Die $\mathrm{Ti} / \mathrm{ZrO}_{2}$-Multilagenproben zeigen sowohl in Mikrokompressionsversuchen als auch in konventioneller Berkovich-Nanoindentation eine gemeinsame Verformung beider Materialien mit einer hohen Duktilität. Es wurden plastische Dehnungen von bis zu mehr als $80 \%$ erreicht. Diese ausgeprägte Duktilität steht in Verbindung mit den Randbedingungen, die durch die Geometrie der Multilagen erzeugt werden. In Abbildung 2.6 sind drei Szenarien der Verformung einer dünnen Metallschicht gezeigt.

Während eine freistehende Schicht, bevor sie versagt, nur bis zu geringen Dehnungen belastet werden kann, ermöglichen die Randbedingungen durch ein unterliegendes Substrat oder die benachbarten Schichten in einer Multilagengeometrie wesentlich höhere Dehnungen.

Für den Fall eines Substrats bzw. benachbarter Schicht muss zwischen einer starken Grenzfläche, an der keine Delamination der Schicht auftritt (b) und einer schwachen Grenzfläche (c) unterschieden werden. Damit sind zusätzlich zu den Randbedingungen, die durch Substrat oder Multilagengeometrie erzeugt werden, auch die Grenzflächeneigenschaften wichtig für den Verformungsmechanismus einer dünnen Metallschicht.

Tritt keine Delamination an der Grenzfläche auf, können während der Verformung Versetzungen in der Metallschicht gespeichert werden und somit zu einer Verformungsverfestigung beitragen. Weiterhin können Spannungskonzentrationen in der Metallschicht abgebaut werden, indem Kräfte in das Substrat abgeleitet werden. Da so die Spannungen homogen in der Schicht verteilt werden, wird die Dehnungslokalisierung bzw. 
Einschnürung wie im Fall der freistehenden Schichten unterbunden. In einer solchen Konfiguration mit starken Grenzflächen können hohe plastische Dehnungen beobachtet werden [Lu07].

Bei nachgiebigen Substraten wird der Effekt der Delokalisierung negativ beeinflusst, sodass die Versetzungen in der Metallschicht an der Grenzfläche die nachfolgenden Versetzungen nicht aufhalten können, was wiederum zu einer lokalen Spannungskonzentration und dem Versagen der Metallschicht führen kann.

Eine Begründung für die Delokalisierung der Dehnungen kann auch in der Mikrostruktur der dünnen Metallschichten gefunden werden. Durch die geringen Schicht- und damit verbunden auch Korngrößen kann die Nukleation von neuen Versetzungen auf einem Gleitsystem unterdrückt werden, wenn auf diesem Gleitsystem bereits Versetzungen vorhanden sind. Die rückwirkenden Spannungen von den im Material gespeicherten Versetzungen sind dann so groß, dass die Nukleation der Versetzungen aufgrund der plastischen Verformung auf einem parallel orientierten Gleitsystem stattfinden muss. Somit werden die Versetzungen homogen über die Schicht verteilt und eine Spannungskonzentration unterdrückt (zum Beispiel [Nix98, Mis03]).

Um die Delamination an der Grenzfläche beispielsweise in einem amorph/kristallinen Multilagensystem zu verhindern, müssen neben den Grenzflächeneigenschaften auch die plastischen Verformungen der beiden Materialien zueinander passen. Ein Beispiel aus der Literatur zeigt, dass an einer Grenzfläche zwischen einem amorphen und einem kristallinen Material genau dort Versetzungen in dem kristallinen Material auftauchen, wo in der amorphen Schicht eine Schertransformationszone (engl. shear transformation zone, STZ) gefunden wird [Wan07].

Eine STZ beschreibt einen Bereich lokaler Neuordnung der Atome, bei der das freie Volumen erhöht wird Spa77, Arg79]. Diese STZ gelten in Verbindung mit den Scherbändern als Träger der plastischen Verformung in amorphen Werkstoffen. Versetzungen und STZ wechselwirken miteinander in der Art, dass Versetzungen STZ triggern können und umgekehrt [Wan07]. Diese gegenseitige Wechselwirkung ermöglicht sowohl die Duktilität der Multilagen-Proben als auch die unterdrückte Delamination der Materialien an den Grenzflächen.

Die Scherbandbildung, die als Versagensmechanismus einer amorphen Schicht angenommen wird, kann bei geringen Schichtdicken der amorphen Komponente unterdrückt werden. Eine Abschätzung der Inkubationslänge eines Scherbandes, welches zur Rissbildung führen kann, liegt bei etwa $100 \mathrm{~nm}$ [Shi06]. Diese kritische Größe für die Scherbandbildung zeigt auf, dass es in dünnen amorphen Schichten, wie sie in den Multilagen vorliegen, dazu kommen kann, dass die Scherbandbildung unterdrückt wird im Vergleich zu massiven Proben.

In der Geometrie des Bruchversuchs ergibt sich jedoch die Möglichkeit, dass die Scher- 


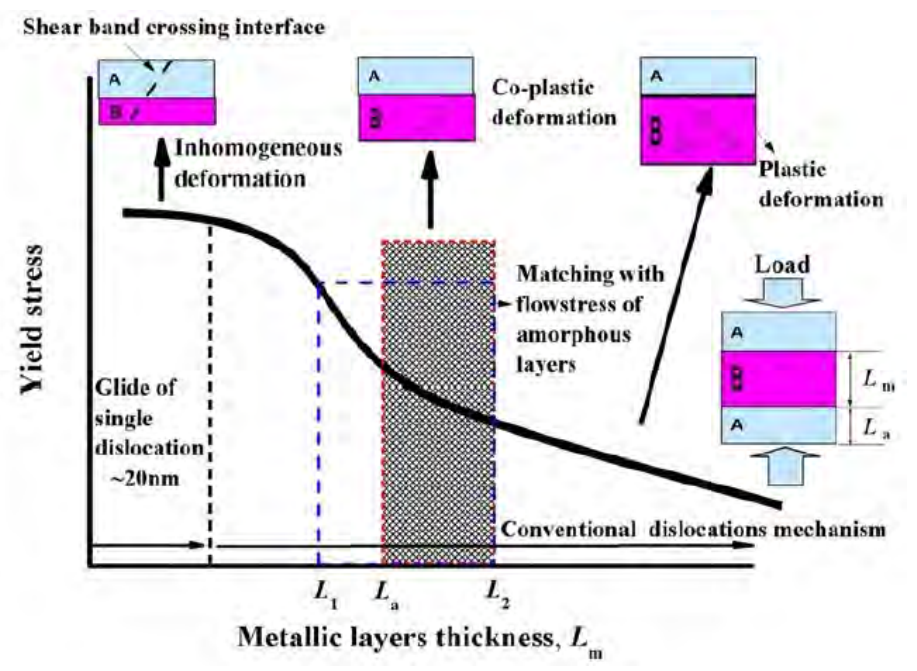

Abbildung 2.7: Verformungsmechanismus kristallin/amorpher Komposite aus [Liu12]

bandbildung trotz der geringen Schichtdicke auftritt. Da es in den TEM-Lamellen freie Oberflächen gibt, die keinen Randbedingungen unterliegen, können diese die Bildung eines Scherbandes ermöglichen. Das Auftreten dieses Effektes konnte anhand von Molekulardynamik-Simulationen nachgewiesen werden [Wan07, Arm11. Auch die Beobachtung der Indentations- und Mikrokompressionsversuche bestätigen diesen Effekt Kno12]. So können am Rand der Säulen, die freie Oberflächen besitzen, Scherbänder beobachtet werden. Entgegengesetzt dazu zeigen die verformten Schichten bei der Indentation oder die Schichten im Inneren der Säule keine Scherbänder, da es keine freien Oberflächen gibt.

Ein Beispiel von $\mathrm{Cu} / \mathrm{CuZr-Schichten} \mathrm{aus} \mathrm{der} \mathrm{Literatur} \mathrm{zeigt} \mathrm{eindeutig} \mathrm{wie} \mathrm{die} \mathrm{Län-}$ genskala - hier die Cu-Schichtdicke - die Verformung der Multilagenstruktur beeinflusst Liu12]. Durch die Variation der Kupferschichtdicke (zwischen $10 \mathrm{~nm}$ und $500 \mathrm{~nm}$ ) konnte gezeigt werden, dass die Verformung der Säulen abhängig von der Kupferschichtdicke ist. In Abbildung 2.7 ist schematisch gezeigt, wie sich der Verformungsmechanismus mit sinkender Kupferschichtdicke von der bevorzugten Verformung der Kupferschicht über die gemeinsame Verformung beider Schichten hin zu einer Verformung dominiert durch Scherverformung in den amorphen Schichten verändert. Gemeinsame Verformung beider Schichten ist dann zu beobachten, wenn beide Schichten gleich dick oder die Cu-Schichten dicker als die CuZr-Schichten sind.

Über ein Modell der Bruchmechanik und die Annahme, dass die Spannungsfelder in der Nähe einer Rissspitze vergleichbar zu dem Spannungsfeld eines Scherbandes ist, kann die plastische Zone $r_{p}$ in Abhängigkeit der Schichtdicke $t_{m}$ der duktilen Komponente bestimmt werden. Es gilt

$$
\begin{gathered}
K_{c}=\sigma_{y s} \sqrt{2 \pi t_{m}} \\
r_{p} \approx 3 \pi \frac{K_{c}^{2}}{\sigma_{y s}^{2}} \approx \frac{2}{3} t_{m}
\end{gathered}
$$




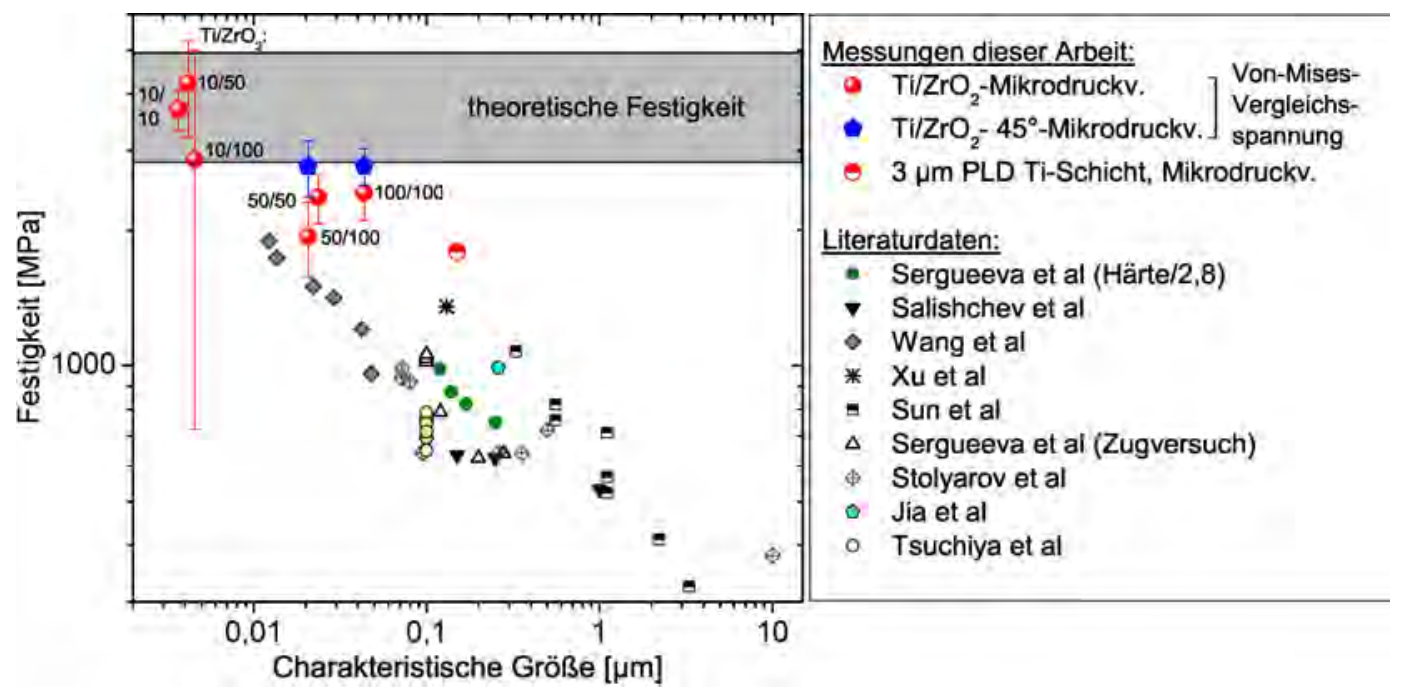

Abbildung 2.8: Vergleich der Festigkeiten der Ti-Schichten mit der Literatur aus Kno12]. Die genauen Literaturverweise sind aus [Kno12] zu entnehmen. Die Festigkeit der $\mathrm{ZrO}_{2}$-Einzelschicht beträgt 6,8 GPa.

Daraus folgt, dass die plastische Zone etwa der Schichtdicke des kristallinen Materials entspricht und ein Scherband nur dann von der kristallinen Schicht in seiner Ausbreitung gestoppt werden kann, wenn die amorphe Schichtdicke geringer ist als die der kristallinen Komponente.

Für die untersuchten Multilagen bedeutet diese Analyse, dass es in keiner der Proben zu erwarten ist, dass die Ti-Schicht ein Scherband in den $\mathrm{ZrO}_{2}$-Schichten aufhalten kann. Diese Erwartung bestätigt sich in den experimentellen Beobachtungen der Bruchversuche sowohl entlang als auch senkrecht zu den Grenzflächen. In der Geometrie entlang der Grenzflächen werden die $\mathrm{ZrO}_{2}$-Schichten stets mit der Ausbildung eines Scherbandes komplett gekreuzt und der Riss wechselt in die benachbarte Ti-Schicht. Die Risse, die senkrecht auf die Grenzflächen zulaufen, überqueren alle Grenzflächen und damit auch alle amorphen oder kristallinen Schichten ohne abgelenkt oder aufgehalten zu werden.

In Abbildung 2.8 sind die aus den gemessenen Daten extrahierten Festigkeiten der TiSchichten in den $\mathrm{Ti} / \mathrm{ZrO}_{2}$-Multilagen gegen die charakteristische Größe im Vergleich mit Literaturdaten gezeigt.

Die Auftragung verdeutlicht, dass mit geringerer charakteristischer Größe der TiSchichten die Festigkeit stetig größer wird. Außerdem ist zu erkennen, dass sowohl die Proben mit den geringsten Ti-Schichtdicken als auch die $45^{\circ}$-Säulen Festigkeiten nahe der theoretischen Festigkeit (DFT-Berechnungen [Oga04]) erreichen.

Die hohen Festigkeiten können entsprechend des in [Kno12] entwickelten Modells durch eine hohe Versetzungsdichte in den Ti-Schichten erklärt werden. Aufgrund der Mikrostruktur der Ti-Schichten treffen viele der an den Korngrenzen in der Schicht nukleierten Versetzungen auf die Grenzfläche zu einer benachbarten amorphen Schicht, die als effektives Hindernis für die Versetzungsbewegung angenommen werden kann. Nur 
wenn die Grenzfläche ein Hindernis für die Versetzungsbewegung ist, kann eine hohe Versetzungsdichte erreicht werden, woraus folgt, dass nicht nur die Ti-Schichtdicke, sondern auch die Grenzflächen entscheidend zu der hohen Versetzungsdichte und damit der hohen Festigkeit beitragen. Eine Untersuchung dünner Aluminiumschichten auf sowohl kristallinen als auch amorphen Substraten bestätigt, dass kristallin/amorphe Grenzflächen ihren rein kristallinen Gegenstücken in Bezug auf die Festigkeit der Grenzflächen überlegen sind Deh02].

\subsection{Stimulationen der Grenzflächen}

In den nachstehenden Abschnitten werden basierend auf Beispielen aus der Literatur verschiedenste Ideen und Taktiken diskutiert, um Grenzflächen und im speziellen die Eigenschaften dieser Grenzflächen zu verändern oder um sie mit externen Anregungen zu stimulieren. Ziel dieser Stimulationen ist es, die Eigenschaften der Grenzflächen so zu beeinflussen, dass das Recycling der Materialien vereinfacht wird, sodass die Materialien effizient und zuverlässig an ihrer gemeinsamen Grenzfläche getrennt werden können. Es werden zum einen Anregungen, die die adhäsiven Eigenschaften der Grenzflächen und der sie bildenden Materialien beeinflussen, und zum anderen Stimulationen des plastischen Verhaltens der Grenzflächen und Materialien behandelt. Weitere Details der hier erwähnten Konzepte und andere Ansätze sind Pio14 zu entnehmen.

\subsubsection{Adhäsionseffekte}

Das adhäsive Zusammenkleben von Materialien an einer Grenzfläche wird durch molekulare Wechselwirkungen an der Kontaktstelle der beiden Materialien hervorgerufen. Es gibt verschiedenste Mechanismen der Adhäsion. So können diffusive, elektrostatische oder dispersive Mechanismen Haftung erzeugen, aber auch mechanische oder chemische Mechanismen sind bekannt. Im Fall der mechanischen Adhäsion werden Poren und Löcher durch das adhäsive Material gefüllt und die Materialien miteinander verbunden. Chemische Adhäsion entsteht durch das Ausbilden von ionischen, kovalenten Bindungen sowie von Wasserstoffbrückenbindungen der Oberflächenatome der Materialien an der Grenzfläche. Die dispersiven Mechanismen beruhen auf der Wechselwirkung der negativen und positiven Ladungen der beteiligten Moleküle, sodass durch die sich ausbildenden Van-der-Waals-Kräfte Adhäsion gewährleistet wird. Zur Ausbildung der diffusiven Adhäsion müssen an der Grenzfläche Atome beider Materialien miteinander gemischt werden. In elektrisch leitenden Materialien können Elektronen verschoben werden, sodass sich eine unterschiedliche elektrische Ladung an der Grenzfläche aufbaut und diese die Verbindung ermöglicht. 


\section{Segregation}

Unter Segregation in Materialien versteht man die Anlagerung bzw. Anreicherung eines bestimmten Bestandteils des Materials im Fall von Legierungen oder die eines anderen Stoffes an internen oder externen Grenzflächen, welche die Oberflächen der Probe darstellen. Zu den internen Grenzflächen werden Korngrenzen, Phasengrenzen, Versetzungen oder Stapelfehler gezählt. An allen diesen Grenzflächen können sich verschiedenste Substanzen anlagern. Diese Segregation ändert die Eigenschaften der Grenzfläche und damit unter Umständen auch das Verhalten der gesamten Probe. So kann es durch Segregation zum Beispiel zum Korngrenzenbruch kommen. Korngrenzen können weiterhin korrodieren oder durch die Anlagerung von Wasserstoff verspröden und unter Belastung versagen [Sea79].

Eingelagerter Wasserstoff in Metalldünnschichten kann hohe Dehnungen erzeugen, die dazu führen können, dass die Metallschicht vom Substrat abgelöst bzw. delaminiert wird Pun03]. Die dort gezeigte Art der Delaminationsexperimente ist universell nutzbar, um die Adhäsionsenergie zu messen. Die Anwendung ist nicht auf Wasserstoff und Metalle (zum Beispiel Niob [Pun04]) beschränkt. Delamination tritt in allen Fällen auf, in denen die erzeugte kompressive Spannung in der Schicht nicht relaxieren kann und die kritische Spannung zur Delamination erreicht wird.

Neben Wasserstoff als lösliche Substanz gibt es weiterhin Experimente in der Literatur mit Schwefel, der sich an Grenzflächen in Nickelaluminiumlegierungen anlagert [Hou05]. Die Segregation ist abhängig vom Aluminiumanteil in der Legierung, da bei höherem Aluminiumanteil weniger oder kein Schwefel angereichert wird. Die Grenzflächenbruchspannung verringert sich mit steigendem Schwefelanteil in der Grenzfläche bzw. Legierung. Schwefel beeinflusst die Adhäsion an der Grenzfläche in zwei Arten. Zum einen wird durch Schwefel die Grenzfläche geschwächt und zum anderen wird die Ausbildung von Poren an der Grenzfläche durch Schwefel in den Nickelaluminiumlegierungen verstärkt. Schwefel erzeugt durch seine Anwesenheit in der Grenzfläche eine Dehnung in dieser Grenzfläche, die zum Versagen führen kann. In Konkurrenz mit dieser Spannung stehen die neugebildeten chemischen Verbindungen durch die Schwefelatome über die Grenzfläche, die das Versagen verhindern Zha03. Auch Metalle wie Kupfer, Platin oder Molybdän können an Metall-Metall-Grenzflächen angereichert werden und die Energie dieser Grenzfläche herabsetzen, wobei zu beachten ist, dass eine Verringerung der Grenzflächenenergie nicht immer mit einer Verringerung der Adhäsion dieser Grenzfläche einhergeht Ger83].

Grenzflächen zwischen zwei unterschiedlichen Materialien, wobei ein Material ein Metall ist, sind häufig sehr stark, sodass der Bruch oft in einem der beteiligten Materialien erfolgt [Eva99]. Segregate oder Verunreinigungen haben, wie bereits aufgezeigt, oft eine versprödende (zum Beispiel Wasserstoffversprödung) und/oder schwächende 
Auswirkung auf die Grenzfläche. So versprödet Kohlenstoff zum Beispiel die Grenzfläche zwischen Saphir und Gold, Schwefel die zwischen Saphir und Nickel, und Silber die zwischen Saphir und Niob. Auch Feuchtigkeit kann Grenzflächen verspröden. Die Grenzflächen Saphir/Gold und Saphir/Nickel neigen unter dem äußeren Einfluss von Feuchtigkeit zur Spannungskorrosion und können reißen oder versagen [Eva99]. Bei der elektrokatalytischen Wasserspaltung entsteht Sauerstoff, der die Grenzfläche zwischen Katalysator und Wasser korrodiert und daher nachträglich in ihren Eigenschaften verändern kann Raa12.

Segregation von Schwefel, Kohlenstoff oder Phosphor an Defekten wie Fehlpassungsversetzungen, Korngrenzen oder Hohlräumen an Metall/Oxid-Grenzflächen führt zur Herabsetzung der Oberflächenenergie des Metalls. Die Segregate stabilisieren daher in einem zweiten Schritt die genannten Defekte und können letztlich die Delamination begünstigen Gra95. Metall/Oxid-Adhäsion ist beispielsweise für die Herstellung von Glühbirnen oder Metall/Oxid-Halbleitern wichtig.

\section{Reaktionen und Phasenübergänge}

Reaktionen der Materialien mit der umgebenden Luftfeuchtigkeit kann einen entscheidenden Einfluss auf das Verhalten des Materials unter Spannung haben. Beispielsweise wird der kritische Spannungsintensitätsfaktor $\left(K_{c}\right)$ für eine Kupfer/SiliciumnitridGrenzfläche um fast $20 \%$ herabgesetzt, wenn die Experimente in Umgebungsluft durchgeführt werden Hir06]. Die höhere Luftfeuchtigkeit beschleunigt das Risswachstum an der Grenzfläche.

Die Bindungslängen an der Grenzfläche zwischen Titannitrid und Vanadium(III)-nitrid können einen Einfluss auf die Adhäsion dieser Grenzfläche haben Yin12]. Die chemische Bindungslänge variiert mit dem Einbau von verschiedensten Materialien. Chrom, Molybdän oder Yttrium setzen beispielsweise die adhäsiven Eigenschaften der Grenzfläche herab. Durch ihre Reaktion an der Grenzfläche werden die chemischen Bindungslängen vergrößert. Den entgegengesetzten Einfluss hat zum Beispiel Scandium, welches die Bindungen schrumpfen lässt und somit die Adhäsion an der Grenzfläche verstärkt.

\section{Veränderungen der Kontaktflächen}

Viele verschiedene Forschergruppen versuchen die einzigartige Eigenschaft von Geckos, sich an Oberflächen jeder Art mit ihren Füßen anzuheften und wieder abzulösen, zu verstehen und nachzuahmen. Nachstehend werden einige Beispiele aufgeführt und erläutert. Eine kontrollierbare Adhäsion, die beliebig in ihrem Zustand verändert werden kann, sodass sie entweder zwei Materialien miteinander verbindet oder nicht, wäre in vielen Bereichen von großem Interesse. Bezogen auf die Idee des Recyclings von Kompositmaterialien wäre eine kontrollierbare Adhäsion eine interessante Variante, um diese 
nach Ende ihrer Lebensdauer zu recyclen. Könnte man die Adhäsion zwischen den beiden Materialien überwinden oder abschalten, wäre das Trennen der Materialien möglich.

An einem Polymerstempel, der in Kontakt mit einem weiteren Material durch die mechanische Verformung kollabiert und dabei seine Kontaktfläche vergrößert, kann ein Stück des Materials durch Adhäsion haften bleiben, wenn die aufgebrachte Dehnung schnell reduziert wird [Wu11, Kim10]. Der Stempel relaxiert und in einem zweiten Schritt wird das angeheftete Materialstück auf einem neuen Substrat abgelegt. Durch die Relaxation verkleinert sich die Kontaktfläche und die Adhäsion reicht nicht mehr aus, um das Materialstück am Stempel festzuhalten, sodass es transferiert werden kann, vergleichbar mit einem Transferdruckprozess. Des Weiteren gibt es druckempfindliche Haftklebstoffe, die ihre Hafteigenschaften durch geringen Druck aufbauen und in besonderen Fällen auch wiederholt verwendet werden können. Temperaturänderungen können zum Verlust der adhäsiven Eigenschaften führen.

Unter Bestrahlung mit Licht können einige Polymere ihre Form temporär verändern. Erst die Verwendung einer bestimmten Wellenlänge des Lichts lässt das Polymer wieder in seine Ausgangsform zurückkommen, vergleichbar zu Formgedächtnismaterialien [Jia06]. Die Polymere können durch das Licht verbogen werden, wachsen und schrumpfen, sodass durch eine solche Änderung entweder die Orientierung oder die Größe der Polymere variiert wird. Diese Veränderungen können die Adhäsionseffekte vergrößern oder verkleinern. Auch Temperaturschwankungen können Orientierungsoder Größenänderungen dieser Formgedächtnispolymere hervorrufen [Red07]. Säulen, bestehend aus diesen Polymeren, werden bei erhöhter Temperatur durch eine extern aufgebrachte Scherspannung verbogen, sodass diese Säulen nicht mehr aufrecht stehen. Eine Temperatur unterhalb der Übergangstemperatur des Polymers lässt die Säulen dann im verkippten Zustand erstarren. In diesem Zustand ist die Kontaktfläche wesentlich kleiner als im Zustand der aufrecht stehenden Säulen, was bedeutet, dass die Adhäsion praktisch ausgeschaltet wird. Durch eine Temperatur oberhalb der Übergangstemperatur kommen die Säulen zurück in den aufrecht stehenden Zustand und die Adhäsion ist wieder eingeschaltet.

Ein ähnlicher Ansatz beruht auf einem externen magnetischen Feld, das als Anregung zu einer Orientierungsänderung von paddelförmigen Nickelproben führt [Nor08]. Diese Paddel orientieren sich durch das Magnetfeld, sodass ihre adhäsive Seitenfläche weg von der Oberfläche einer Probe zeigt, die angeheftet werden soll. Durch die Rotation wird die Kontaktfläche für die Adhäsion verringert, sodass das adhäsive Anheften nicht möglich ist. Ohne Magnetfeld drehen sich die Paddel wieder zurück in ihre Ausgangsform. Diese Rotation ist reversibel, was es ermöglicht, beliebig oft zwischen adhäsivem und nicht adhäsivem Kontakt hin und her zu schalten.

Wie bereits aufgezeigt, hat die Größe der Kontaktfläche einen entscheidenden Einfluss 
auf die Stärke der Adhäsion. Ein dementsprechend schlüssiger Ansatz zur Erhöhung der Adhäsion is,t die Kontaktfläche in viele kleine, unabhängige Kontaktflächen zu unterteilen und somit eine effektiv größere Kontaktfläche zu erreichen Arz03. Diese Idee begründet sich in der Natur. Tiere mit größerem Körpergewicht brauchen kleinere bzw. feinere Haare an ihren Füßen und damit auch mehr adhäsive Kontaktflächen, um an Wänden oder unter Decken laufen zu können. Bei Käfern sind die adhäsiven Flächen mehrere Mikrometer groß, wobei bei den schon erwähnten Geckos die feinen Haare an ihren Füßen nur wenige hundert Nanometer groß sind. Kontaktflächen, in viele kleine Flächen zu unterteilen, erhöht Adhäsion. Eine Untersuchung an Säulen aus Polydimethylsiloxan ( $(\underline{\mathrm{PDMS}})$ mit verschiedenen Durchmessern und Abständen zeigt eindeutig, dass die Kraft zum Trennen des Kontakts steigt, wenn mehr Säulen in Kontakt sind Lee07].

\subsubsection{Einfluss plastischer Energie auf Sprödbrüche}

Im Folgenden werden Möglichkeiten, plastische Eigenschaften von Materialien zu beeinflussen, diskutiert. Als erste wird hier der Übergang eines Materials von duktilem zu sprödem Verhalten genannt. Dieser Übergang ergibt sich durch Temperaturveränderungen. So kann ein Material, das sich bei Raumtemperatur duktil verformt, bei niedrigeren Temperaturen spröde verformen. Für Temperaturen oberhalb der Raumtemperatur kann die Versetzungsaktivität während einer Verformung zunehmen. Diese Zunahme reicht bis zu einer bestimmten Temperatur, bei der sich Versetzungen so um eine sich ausbreitende Rissspitze anordnen, dass diese die extern angelegte Spannung nicht spürt, daher $K_{c}$ nicht erreicht wird und der Riss nicht weiter wächst. Diese Temperatur bestimmt dann die Übergangstemperatur von duktilem zu sprödem Verhalten. Grenzflächen zwischen duktilen und spröden Materialien zeigen im Fall von Gold und Saphir, dass der Riss sich durch Duktilität im Metall ausbreitet Tur96, Rei90, Rei91. Wird diese Duktilität im Metall durch einen Duktil-Spröd-Übergang unterdrückt, sollte sich der Risswachstumsmechanismus verändern, sodass der Riss möglicherweise entlang der Grenzfläche wächst. Im Fall von Gold/Saphir kann der Risswachstumsmechanismus durch Luftfeuchtigkeit geändert werden.

Weitere Untersuchungen zeigen, dass die Plastizität einen entscheidenden Einfluss auf die Spannungen in der Nähe der Rissspitze sowie die möglichen Wege des Risswachstums in der Nähe von Grenzflächen haben kann [Til05]. Der Widerstand zur Rissbildung einer Probe ist abhängig von der Energie, die in der Nähe der Rissspitze dissipiert wird Dau98. Die Größe des Bereichs, in dem Energie dissipiert wird, ist wiederum abhängig von der Plastizität im duktilen Material an der Grenzfläche. Anhand dieser Beispiele ist es denkbar, dass sich Grenzflächen zwischen Materialien, die sich in ihrem Verformungsverhalten unterscheiden, durch einen Duktil-Spröd-Übergang in geeigne- 
ter Weise beeinflussen lassen, sodass das Risswachstum hin zur Grenzfläche verschoben werden kann.

Die plastischen Eigenschaften, die wie gezeigt wichtig für die Rissausbreitung sind, können weiterhin beispielsweise durch die Behinderung der Versetzungsbewegung verändert werden. In amorphen Silicium, das auf Aluminium deponiert ist und gedehnt wird, können Risse stoppen oder diskontinuierlich wachsen [Hac91]. Dieses Verhalten steht in Verbindung mit der Mikrostruktur des kristallinen Aluminiumsubstrats. So werden Versetzungen an der Grenzfläche aufgestaut und die unterschiedlichen kristallographischen Orientierungen der Aluminiumkörner beeinflussen stark die Richtung der Rissausbreitung im Silicium. Wenn die notwendige Spannung zur Bewegung von Versetzungen in Metallen nicht hoch genug ist, dass sich die Versetzungen bewegen können, werden die Metalle mittels Rissbildung und -wachstum versagen, sodass sie eher sprödes Verhalten zeigen. Durch starke Dehnungen können ursprünglich amorphe Materialien kristallisieren, was folglich die elastischen und plastischen Eigenschaften ändert. Wie bereits erwähnt, kann durch die Unterteilung der Kontaktflächen die Adhäsion vergrößert werden. Ein ähnlicher Größeneffekt tritt für Komposite aus verschiedenen Stählen auf [no08]. Mit dünnerer Schichtdicke des spröden Stahls ergibt sich ein Übergang vom Sprödbruch hin zu einem duktilen Abscheren entlang der Grenzfläche. Die genannten verschiedenen Effekte, wie sich Eigenschaften von Materialien verändern können, eröffnen Möglichkeiten, wie eine Grenzfläche designt und wie gewünscht durch externe Stimuli beeinflusst werden kann. 


\section{Experimentelle Methoden}

Im folgenden Kapitel dieser Arbeit werden die verwendeten Geräte, Proben sowie die notwendigen Präparationsschritte und die eigentliche Bruchversuchsmethode vorgestellt und beschrieben. Von zentraler Bedeutung für diese Arbeit sind hier das Tranmissionselektronenmikroskop (engl. transmission electron microscope, TEM) und der TEM-STM-Probenhalter (scanning tunneling microscope, STM), deren Funktionsweise nachfolgend besprochen werden. Weiterhin wird die Entwicklung einer neuartigen und geeigneten Geometrie zur Beobachtung der Rissbildung und des Bruches in-situ innerhalb des TEM und abschließend die Präparation der Proben für die in-situ Bruchversuche dargestellt. Des Weiteren werden sowohl die Auswertung der Bruchversuche als auch unterstützende Simulationen mittels der Finite-Elemente-Methode (FEM) beschrieben.

\subsection{Verwendete Geräte}

\subsubsection{IonenstrahImikroskop}

Das in dieser Arbeit verwendete Ionenstrahlmikroskop (engl. focused ion beam, FIB) Nova 600 NanoLab von FEI ist ein sogenanntes Dual-Beam-Mikroskop, was bedeutet, dass neben dem Ionenstrahl (Gallium-Ionen) auch ein Elektronenstrahl zur Bildgebung zur Verfügung steht. Dadurch kann das FIB auch als konventionelles Rasterelektronenmikroskop (engl. scanning electron microscope, SEM) genutzt werden. Auf die grundlegende Funktionsweise eines Rasterelektronenmikroskops wird hier nicht weiter eingegangen, da das SEM in dieser Arbeit nur zur Kontrolle der Präparationsschritte genutzt und auf Haf07 für eine detaillierte Beschreibung eines SEM verwiesen wird. Zur Abbildung mit dem Ionenstrahlmikroskop werden, ähnlich wie im SEM, die inelastischen Wechselwirkungen der Ionen mit den Elektronen der Oberflächenatome 
und der dadurch erzeugten niederenergetischen Sekundärelektronen verwendet. Neben der Abbildung ist es möglich, die hochenergetischen Ionen zu nutzen, um Atome aus der Probe herauszulösen. Die Stromstärke des Ionenstrahls bestimmt die Anzahl der Ionen, die pro Zeiteinheit auf die Probenoberfläche auftreffen und damit auch das Volumen, welches aus der Probe herausgelöst wird. Die Größe des Volumens ist jedoch weiterhin abhängig, zum Beispiel von dem Probenmaterial und dessen Kristallstruktur und -orientierung. Definierte Muster (engl. pattern) werden vom Ionenstrahl zielgenau abgerastert und die Probe entsprechend bearbeitet. Mit der Hilfe eines Mikromanipulators (Omniprobe) und eines Gasinjektionssystems können so kleine Stücke einer massiven Probe heraus präpariert werden und auf spezielle Probenhalter für weitergehende TEM-Untersuchungen transferiert werden.

In dem hier verwendeten Gerät sind Elektronen- und Ionenstrahl um einen Winkel von $52^{\circ}$ zueinander verkippt. Befindet sich die Probe in der euzentrischen Höhe, so kann unabhängig vom Kippwinkel mit beiden Strahlen die identische Stelle auf der Probe beobachtet werden, was das Arbeiten immens erleichtert. Die Beschleunigungsspannung der Ionen liegt bei $30 \mathrm{kV}$, die der Elektronen bei $5 \mathrm{kV}$. Die Ionenstrahlströme können zwischen minimal $1 \mathrm{pA}$ bis maximal $20 \mathrm{nA}$ gewählt werden. Das FIB wird in dieser Arbeit zur Präparation der Proben für die Bruchversuche (vgl. Abschnitt 3.3.1) und für Querschnitts-TEM-Lamellen genutzt.

\subsubsection{Transmissionselektronenmikroskop}

In der Transmissionselektronenmikroskopie werden die Proben im Gegensatz zur Rasterelektronenmikroskopie mit hochenergetischen Elektronen durchstrahlt. Aufgrund der starken Wechselwirkungen zwischen Elektronenstrahl und Probenatomen darf die Dicke der Probe nicht größer als einige $100 \mathrm{~nm}$ sein, wobei für spezielle Analysemethoden und Probenmaterialien (schwere Elemente) die Dicke der Probe sogar geringer als $100 \mathrm{~nm}$ sein sollte, um qualitativ hochwertige Aufnahmen zu erhalten. Bezüglich des Funktionsprinzips unterscheiden sich ein TEM und ein Lichtmikroskop nicht grundlegend. Mehrere hintereinander geschaltete Linsen bilden das Objekt bzw. die Probe mit der gewünschten Vergrößerung auf dem Schirm bzw. einem CCD-Sensor ab. Die Verwendung hochenergetischer Elektronen, deren Quellen geheizte Kathoden (Wolfram- bzw. Lanthanhexaboridkathoden) oder Feldemissionskathoden sind, ermöglichen eine geringere Wellenlänge im Vergleich zu Licht und dadurch ein höheres Auflösungsvermögen. Die Elektronen werden mit Spannungen von mehreren $100 \mathrm{kV}$ beschleunigt und durch magnetische Linsen als Analogon zu den Glaslinsen im Lichtmikroskop fokussiert. Diese magnetischen Linsen besitzen im Vergleich zu Glaslinsen jedoch größere Aberrationen, welche das Auflösungsvermögen des Mikroskops negativ beeinflussen. Die Wechselwirkungen mit der Probe sind hauptsächlich von elastischer 
Natur. Aufgrund des Welle-Teilchen Dualismus können Elektronen sowohl als Teilchen als auch als Wellen interpretiert werden, sodass mit der Bragg-Bedingung bzw. den Laue-Gleichungen die Interferenz der Elektronen am Kristallgitter berechnet werden kann. Die kohärent elastisch am Kristallgitter der Probe gestreuten Elektronen können für die Untersuchung der Kristallografie des Objekts genutzt und Beugungsbilder aufgenommen werden. Neben diesem Betriebsmodus, in dem die Beugungsinformationen abgebildet werden, können im Abbildungsmodus des Mikroskops Hell- und Dunkelfeldaufnahmen (engl. bright field - BF, dark field micrograph DF) aufgenommen werden. Zusätzlich ermöglicht die Hochauflösende Transmissionselektronenmikroskopie (engl. high resolution transmission electron microscopy, HRTEM) die Analyse der kristallographischen Struktur mit Sub-Ångström-Auflösung. Weitere Details zur Funktionsweise eines TEM sind in der Literatur zu finden Haf11, Wil09.

Für die transmissionselektronenmikroskopischen Untersuchungen dieser Dissertation werden ein Philips CM30 sowie ein CM12 und ein FEI Titan 80 - 300 ETEM G2 verwendet. Die Beschleunigungsspannungen dieser Mikroskope liegt zwischen $120 \mathrm{kV}$ und $300 \mathrm{kV}$. Das atmosphärische Transmissionselektronenmikroskop (engl. environmental transmission electron microscope, ETEM) und dessen Funktionsweise sowie die speziellen Einsatzmöglichkeiten werden im folgenden Abschnitt dargestellt.

\section{ETEM}

Bei dem in dieser Arbeit verwendeten ETEM handelt es sich um ein modernes, hochauflösendes FEI Titan Mikroskop, welches zusätzlich die Möglichkeit bietet, in der direkten Umgebung der Probe verschiedene Gasatmosphären zu realisieren. Als Gase sind zum Beispiel Wasserstoff, Sauerstoff, Stickstoff, Kohlenmonoxid oder Wasserdampf mit verschiedenen Drücken wählbar. Der maximal erlaubte Druck ist für Stickstoff $p_{\mathrm{N}_{2}}=20$ mbar. Des Weiteren können die Gase auch miteinander gemischt und in den Probenraum des ETEM eingelassen werden.

Ein differentielles Vakuumsystem mit verschiedenen Pumpstufen und Pumpaperturen ermöglicht das Einlassen von Gasen in das ETEM. Auch wenn im Bereich der Probe ein Gasdruck herrscht, muss gewährleistet sein, dass im Bereich der Feldemissionselektronenquelle weiterhin Hochvakuumbedingungen vorzufinden sind. In Abbildung 3.1 ist ein Querschnitt durch ein relativ baugleiches ETEM gezeigt. Es sind die verschiedenen Turbomolekularpumpen (TMP), die das differentielle Vakuum in der Säule erzeugen, dargestellt. Das Gas im Probenraum kann mit Hilfe eines Restgasanalysators (RGA) untersucht und der Probenraum mittels eines Plasmareinigers gereinigt werden. Im Vergleich zu Probenhaltern, die einen Gasfluss direkt an der Probe ermöglichen, bietet das ETEM durch das differentielle Vakuumsystem den entscheidenden Vorteil, dass verschiedenste Probenhalter für die Untersuchungen unter Gasatmosphären genutzt 


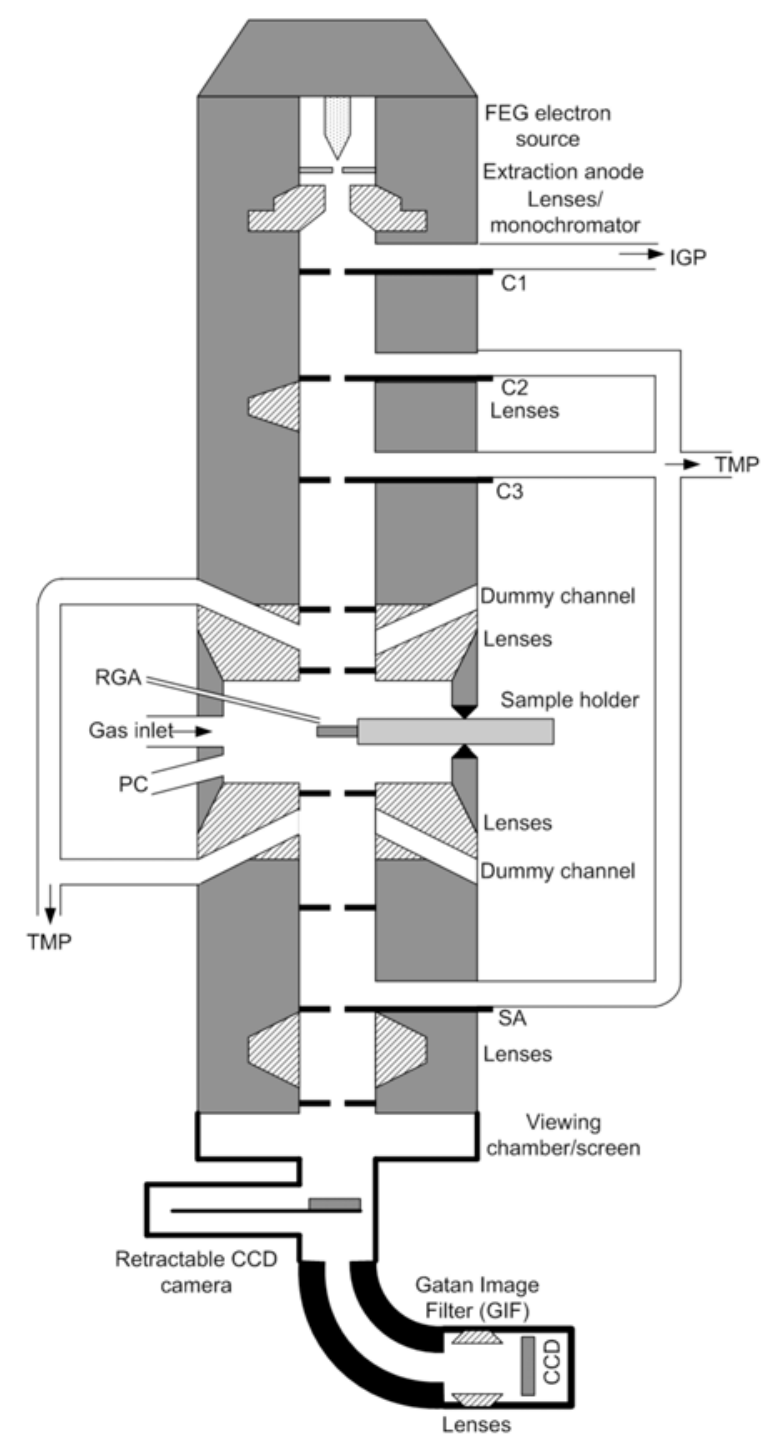

Abbildung 3.1: Schematische Querschnittszeichnung eines relativ baugleichen ETEM (Standort: Center of Electron Nanoscopy, Technical University of Denmark) [Han10]

werden können [Wag12, Han10].

Des Weiteren verfügt das verwendete Mikroskop am Institut für Materialphysik der Georg-August-Universität Göttingen über einen Monochromator und einen Korrektor der sphärischen Aberrationen im Abbildungssystem. Der Monochromator reduziert die Schwankungen der Elektronenenergien auf Werte geringer als $0,21 \mathrm{eV}$, was für diese Arbeit nicht weiter genutzt wird. Der Bildkorrektor hingegen ermöglicht eine Verbesserung der Auflösung des Mikroskops. Das Informationslimit beträgt für das verwendete Mikroskop im ETEM-Modus bei 0,5 mbar Stickstoff 0,12 nm und ohne Gasatmosphäre $\leq 0,10 \mathrm{~nm}$ [FEI13]. 


\section{Analytische Methoden}

Neben der konventionellen Mikroskopie kann die Probe mit einem sehr stark fokussierten Elektronenstrahl abgerastert werden. In der Rastertransmissionselektronenmikroskopie (engl. scanning transmission electron microscopy, STEM) werden für hochaufösende Aufnahmen Strahldurchmesser kleiner als $\leq 0,1 \mathrm{~nm}$ benötigt. Der Durchmesser des Strahls ist limitiert durch die Aberrationen des optischen Systems, die umso wichtiger sind, je weiter der Strahl von der optischen Achse entfernt ist. Weiterhin muss trotz des geringen Strahldurchmessers ein hinreichend großer Strahlstrom erreicht werden. Dieser ist abhängig von der Anzahl der kohärenten Elektronen, die in der Elektronenquelle erzeugt werden.

Vergleichbar zur konventionellen Mikroskopie können Hell- oder auch Dunkelfeldbilder durch die geeignete Auswahl der transmittierten Elektronen erzeugt werden. Die ringförmigen Dunkelfelddetektoren für den STEM-Betrieb sind konzentrisch um die optische Achse des Mikroskops angeordnet und werden als annulare Dunkelfelddetektoren bezeichnet. Die unter höheren Winkeln gestreuten Elektronen enthalten die chemische Information der Elemente, an denen sie abgelenkt werden. Die Intensität der gestreuten Elektronen ist proportional zum Quadrat der Ordnungszahl (Z), sodass Abbildungen unter Verwendung dieser Elektronen auch Z-Kontrastaufnahmen (engl. high-angle annular dark field, HAADF) genannt werden. Durch die Möglichkeit, den Strahl lokal an designierte Positionen oder entlang bestimmter Muster zu bewegen, können im STEM-Betrieb mit zusätzlichen Detektoren quantitative Daten gesammelt werden. In dieser Arbeit wird ausschließlich die Elektronenenergieverlustspektroskopie (engl. electron energy loss spectroscopy, EELS) als quantitative Charakterisierungsmethode verwendet.

EELS-Spektren enthalten Informationen über die chemischen Elemente einer Probe, zusätzlich können die chemischen Bindungen und auch die elektronische Struktur auf der atomaren Skala analysiert werden. Wird eine Probe mit Elektronen einer definierten kinetischen Energie bestrahlt, so können die Elektronen sowohl elastisch als auch inelastisch in der Probe gestreut werden. Bei der inelastischen Streuung verlieren die einfallenden Elektronen einen Teil ihrer Energie und werden von ihrer ursprünglichen Ausbreitungsrichtung abgelenkt. Der Energieverlust wird mit einem Spektrometer gemessen und ermöglicht Rückschlüsse auf die Ursachen des Verlusts. Die Energie, die durch die Ionisation der Atome der Probe verloren geht, kann dazu genutzt werden, um charakteristische Ionisationskanten der untersuchten Materialien zu bestimmen. Im Spektrometer werden die inelastisch gestreuten Elektronen mit einem magnetischen Prisma durch die Lorentzkraft unterschiedlich stark abgelenkt. Elektronen, die wenig Energie verloren und damit noch den Großteil ihrer kinetischen Energie vor dem Durchgang der Probe haben, werden weniger stark abgelenkt, als Elektronen mit höherem 
Energieverlust. Diese abgelenkten Elektronen werden mit elektromagnetischen Linsen als Spektren abgebildet. Durch die Verwendung von mechanischen Schlitzen innerhalb des Spektrometers können spezifische Bereiche des EELS-Spektrums ausgewählt und damit Bilder erzeugt werden, die die Verteilung eines speziellen Elements ortsaufgelöst widerspiegelt. Diese Abbildungsmethode wird energiegefilterte Transmissionselektronenmikroskopie (engl. energy filtered transmission electron microscopy, EFTEM) genannt. Weiterhin können EELS-Spektren zur Bestimmung der Dicke $t$ der Probe entlang der Durchstrahlrichtung der Elektronen genutzt werden. Die Dicke wird aus den Verhältnissen der Integrale über das komplette Spektrum $I_{t}$ und über das NullVerlust-Maximum (engl. zero-loss peak, ZLP) $I_{0}$ bestimmt. Der Proportionalitätsfaktor zwischen diesem Verhältnis und der Dicke ist die freie Weglänge der Elektronen $\lambda$ im Material, die berechnet werden kann Mal88.

$$
t=\lambda \ln \frac{I_{t}}{I_{0}} \quad \text { mit } \quad \lambda=\frac{106 F\left(E_{0} / E_{m}\right)}{\ln 2 \beta E_{0} / E_{m}}
$$

Der mittlerer Energieverlust $E_{m}=7,6 Z^{0,36}$ berechnet sich aus der mittleren Ordnungszahl der Probe, insofern sie aus verschiedenen Elementen besteht.

\section{EELS-Analyse der Grenzflächen}

Im STEM-Modus des TEM wird der Elektronenstrahl senkrecht zu einer Grenzfläche entlang einer Linie über die Grenzfläche geführt. In einem räumlich definierten Abstand entlang der Linie werden Spektren aufgenommen. Die Analyse dieser Spektren liefert die Konzentrationsprofile der jeweiligen Atomsorten entlang der Bewegung des Elektronenstrahls über die Grenzflächen hinweg. Die räumliche Auflösung aller aufgenommenen EELSłLinienaufnahmen ist $0,5 \AA$. Es werden für alle Proben die charakteristischen Energieverluste von $\mathrm{Ti}, \mathrm{O}$ und $\mathrm{Zr}$ aufgenommen und ausgewertet. Im Fall von Ti und Zr werden jeweils die Übergänge der L-Schalen und für O die K-Übergänge analysiert.

Alle Spektren sind im sogenannten Dual-EELS-Mode aufgenommen, was bedeutet, dass zu den Spektren mit den materialspezifischen Informationen bei höheren Energieverlusten auch noch jeweils das Null-Verlust-Maximum (engl. zero-loss peak, ZLP) aufgezeichnet wird. Anhand des ZLP kann eine mögliche Verschiebung der Spektren auf der Energieskala beobachtet und entsprechend korrigiert werden. Zur Korrektur dieser Verschiebung wird eine Gaußsche Verteilungsfunktion an das ZLP angepasst und anhand der Lage der Gaußschen Verteilungsfunktion relativ zum Ursprung der Energieskala werden alle Spektren um den entsprechenden Energiewert verschoben.

Durch die Verwendung eines Plasmareinigers und einer mit flüssigen Stickstoff gefüllten Kühlfalle am TEM kann eine Kontamination der Probe während der Aufnahme der Spektren nahezu ausgeschlossen werden. Typischerweise ist zu beobachten, dass 


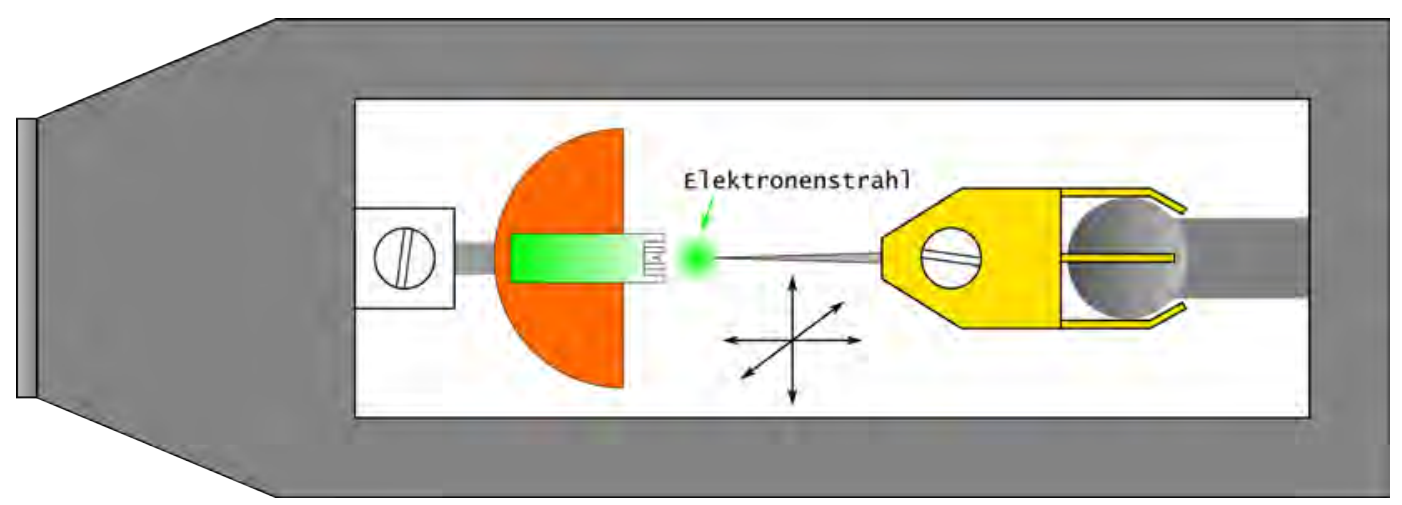

Abbildung 3.2: Skizze des STM-TEM-Probenhalters mit dem Hut inklusive der Spitze (rechts) und gegenüberliegend die Probe fixiert mit Hilfe eines dünnen Kupferdrahtes

durch die Wechselwirkung mit dem stark fokussierten Elektronenstrahl im STEMModus Kohlenstoff auf der Probenoberfläche abgelagert wird, welcher die Qualität der EELS-Daten negativ beeinflusst.

\subsubsection{TEM-STM-Probenhalter}

In dieser Arbeit wird der TEM-STM-Probenhalter der Firma nanofactory ${ }^{\mathrm{TM}}$ Instruments verwendet, wobei die STM-Funktion nicht weiter genutzt wird. Grundsätzlich kann über die Tunnelspitze eine Spannung zwischen Spitze und Probe angelegt werden und der entsprechend erzeugte Tunnelstrom wie in einem konventionellen STM gemessen werden. Der Halter ist als Einfachkipp-Halter ausgeführt, sodass nur die Rotationsachse des Goniometers des Mikroskops genutzt werden kann, um die Probe auszurichten. Für diese Arbeit wird ausschließlich die Möglichkeit der präzisen Positionierung der Spitze genutzt. In Abbildung 3.2 ist eine Skizze des vorderen Endes des Halters gezeigt. Auf der linken Seite wird die Probe mit einem dünnen Kupferdraht mit einem Durchmesser von ca. $200 \mu \mathrm{m}$ befestigt. Zur Befestigung wird der Draht mit einer Madenschraube mechanisch im Halter fixiert, dies dient gleichzeitig zur elektrischen Kontaktierung. Am Kupferdraht ist ein speziell hergestelltes halbkreisförmiges Kupferblech angeklebt. Die weitere Probenpräparation wird in Abschnitt 3.3.1 beschrieben. Gegenüberliegend befindet sich ein Piezo-Aktuator, an dessen Ende eine kleine Kugel montiert ist. Über diese Kugel wird ein Hut mit der Rastertunnelspitze geschoben. Der Piezo-Aktuator verfügt über einen groben und einen feinen Verfahrmodus. Im Grobmodus wird der Piezo-Aktuator lediglich geschüttelt und der Hut gleitet über die Oberfläche der Kugel in die gewünschte Bewegungsrichtung. Um dieses Abgleiten zu ermöglichen, ist die Kugel mit einer speziellen Schicht überzogen, die zusätzlich auch die elektrische Leitfähigkeit realisiert. Im Feinmodus wird der Piezo-Aktuator kontinuierlich bewegt in alle drei Dimensionen einzeln gesteuert. Die maximale Bewegung ist im Grobmodus in allen Dimensionen $2 \mathrm{~mm}$. Im Feinmodus kann die Spitze entlang der 


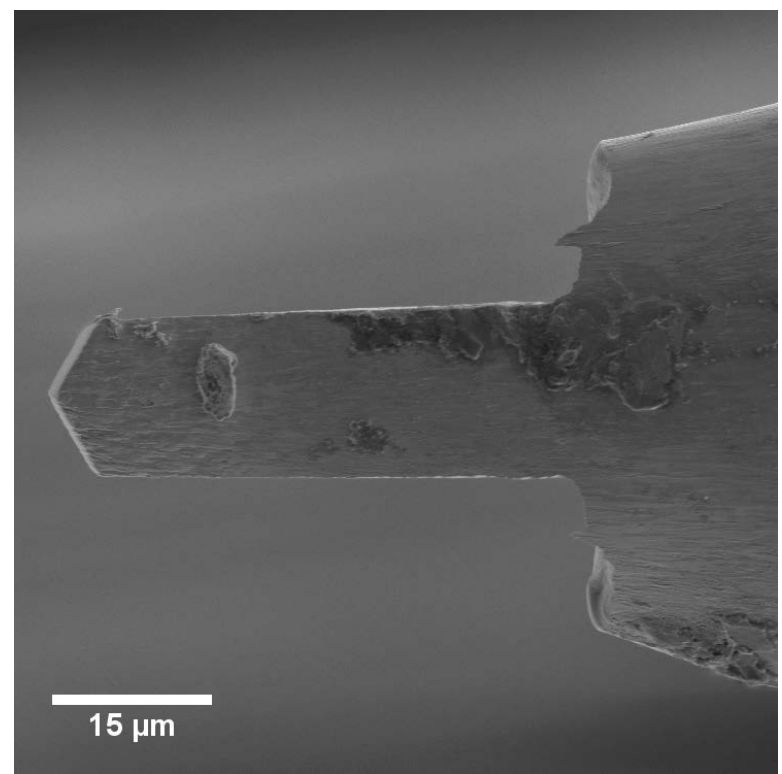

Abbildung 3.3: SEM Aufnahme der keilförmigen Spitze, wie sie in den in-situ Bruchversuchen genutzt wird

Rotationsachse des Halters ca. $2 \mu \mathrm{m}$ auf die Probe zu bewegt werden. Die Auflösung der Bewegung in dieser Richtung ist $0,025 \AA$ und in den beiden anderen Richtungen jeweils $0,2 \AA$.

Als Spitze wird in dieser Arbeit ein Wolframdraht genutzt, der mit dem FIB in eine Keilform mit einem Öffnungswinkel von $120^{\circ}$ gebracht wird, wie in Abbildung 3.3 zu sehen ist. Diese Spitze wird auf der beweglichen Seite des Halters eingebaut. Die genauere Beschreibung des Bruchversuchs erfolgt in Abschnitt 3.3.2. Durch die hier gewählte Anordnung von Spitze und Probe wird die hohe Flexibilität des Halters gut genutzt, Probengeometrie und auch die Spitze können so einfach und effizient unabhängig voneinander variiert werden.

\subsection{Proben}

Das in dieser Arbeit verwendete Materialsystem besteht aus einer kristallinen und einer amorphen Komponente, wobei als kristallines Material polykristallines Titan (Ti) und als amorphe Komponente Zirkoniumdioxid $\left(\mathrm{ZrO}_{2}\right)$ gewählt wurde. Die Proben wurden als Schichtpakete mit unterschiedlichen Einzelschichtdicken mit Hilfe der gepulsten Laserdeposition (engl. pulsed laser deposition, PLD) hergestellt. Die Schichtpakete sind von Dr. Tobias Liese in der Arbeitsgruppe von Prof. Dr. Hans-Ulrich Krebs im Rahmen seiner Diplom- sowie Doktorarbeit [Lie07, Lie11, Lie12] am Institut für Materialphysik an der Georg-August-Universität Göttingen hergestellt worden.

Bei der Schichtherstellung mittels PLD wird eine Targetoberfläche mit einem intensiven 


\begin{tabular}{lccc}
$t_{\mathrm{ZrO}_{2}} \backslash t_{\mathrm{Ti}}$ & $10 \mathrm{~nm}$ & $50 \mathrm{~nm}$ & $100 \mathrm{~nm}$ \\
\hline \hline $10 \mathrm{~nm}$ & $\mathrm{C}$ & & \\
$50 \mathrm{~nm}$ & $\mathrm{D}$ & $\mathrm{A}$ & \\
$100 \mathrm{~nm}$ & $\mathrm{E}$ & $\mathrm{F}$ & $\mathrm{G}$
\end{tabular}

Tabelle 3.1: Übersicht der hergestellten $\mathrm{Ti} / \mathrm{ZrO}_{2}$-Proben mit unterschiedlichen Schichtdicken

Laserstrahl beleuchtet. Die Abtragung der Atome aus dem Target erfolgt weitestgehend stöchiometrisch. Die herausgelösten Atome werden stark beschleunigt und können auf einem entsprechend ausgerichteten Substrat als Schicht deponiert werden.

Als Targetmaterialien wurden polykristallines $\mathrm{Ti}$ mit einer Reinheit von 99,99\% und polykristallines $\mathrm{ZrO}_{2}$ (kubisch teilstabilisiert mit $3 \mathrm{~mol} \% \mathrm{Y}_{2} \mathrm{O}_{3}$ ) verwendet. Der Basisdruck in der Vakuumkammer betrug während des Beschichtungsvorgangs ca. $10^{-7}$ mbar. Alle Proben wurden auf Stücken von (111)-orientierten $\mathrm{SiO}_{x}$-Wafern abgeschieden. In Tabelle 3.1 ist eine Übersicht über die hergestellten Proben mit den jeweiligen Schichtdicken gezeigt. Zusätzlich zu den Schichtpaketen wurden Einzellagen der beiden Komponenten in den Multilagen hergestellt. Für Ti und $\mathrm{ZrO}_{2}$ wurden dünne Filme mit einer Schichtdicke von jeweils ca. $3 \mu \mathrm{m}$ präpariert. Für alle weiteren Details des Herstellungsprozesses sei auf [Lie07, Lie12] verwiesen.

Im Folgenden werden die verschiedenen Proben anhand der Schichtdicken der Einzelkomponenten unterschieden und benannt. Es wird die nachstehenden Nomenklatur verwendet. Die Bezeichnung Ti100/ZrO 100 bezieht sich auf die Probe "Gïn Tabelle 3.1 mit jeweils $100 \mathrm{~nm}$ Einzelschichtdicke beider Materialien. Entsprechend werden alle anderen Proben durch die Angabe der Schichtdicke nach dem jeweiligen Material charakterisiert.

\subsection{In-situ Bruchversuch}

Das Ziel des in dieser Arbeit neu entwickelten Bruchversuchs ist, die Rissbildung und -propagation im TEM beobachten und analysieren zu können. Durch die Möglichkeit, den sich bildenden Riss zu beobachten, kann die plastische Zone während der Rissbildung und -bewegung direkt ausgemessen werden, was in den standardisierten ex-situ Experimenten meist nicht möglich ist. Die Realisierung dieses Versuchs innerhalb eines TEM liefert zusätzliche Informationen für die mikroskopischen Mechanismen des Bruchverhaltens nanoskaliger Probensysteme. In den folgenden Abschnitten werden sowohl die notwendigen Präparationsschritte der beiden verwendeten Geometrien als auch der eigentliche Bruchversuch im TEM beschrieben. 


\subsubsection{Präparation der Geometrien}

Nachdem die Multilagen gemäß Abschnitt 3.2 hergestellt wurden, wird abschließend auf alle Proben eine Silicium-Deckschicht deponiert. Diese Deckschicht ist für die Geometrie des Bruchversuchs notwendig (siehe Abs. 3.3.2). Die Schicht wird mittels Ionenstrahlsputtern ausgehend von einem polykristallinen Silicium-Target hergestellt (Depositionstemperatur: $23^{\circ} \mathrm{C}$ bis $150^{\circ} \mathrm{C}$, Leistung: $20 \mathrm{~mW}$, Sputtergas Xe bei $1 \times 10^{-4}$ mbar, Kammerbasisdruck: $7 \times 10^{-4}$ mbar). Die Schichtdicken liegen zwischen $1,5 \mu \mathrm{m}$ und $3 \mu \mathrm{m}$.

Die Abbildungen 3.4 und 3.6 zeigen die verschiedenen Präparationsschritte der Proben für die beiden realisierten Bruchversuchsgeometrien.

\section{Parallele Ausrichtung}

Nachfolgend werden die einzelnen Schritte für die Geometrie der Rissausbildung entlang der Grenzflächen genauer erläutert. Nach dem Aufbringen der Si-Deckschicht werden die Proben in dünne Streifen mit einer Dicke von ca. $100 \mu \mathrm{m}$ bis $200 \mu \mathrm{m}$ gesägt (vgl. Abbildung 3.4a). Es wird eine kommerzielle Wafersäge (Disco DAD-321) verwendet. Die Streifen werden anschließend in eine Keilform geschliffen und mechanisch poliert (Abbildung 3.4b). An dem dünneren Ende dieses Keils entsteht in den weiteren Schritten die Lamelle für die Bruchversuche.

Für die Präparation mit dem FIB und für den späteren Einbau in den TEM-STMProbenhalter werden die keilförmigen Probenstücke auf halbkreisförmige Kupferhalter aufgeklebt. Der dünnere Teil des Keils ragt über die gerade Kante des Halbkreises hinaus, sodass dort mit dem FIB weiter präpariert werden kann (vgl. Abbildung 3.4c). Essenziell wichtig ist die Ausrichtung der Multilagen relativ zur geraden Kante des Halbkreises. Die Multilagen sollten senkrecht zur Kante stehen, um die bestmögliche Ausrichtung von STM-Spitze und Multilagen im TEM zu erreichen. Eine senkrechte Ausrichtung der Multilagen vereinfacht außerdem die Präparation der Lamellen, die nachstehend beschrieben wird.

Mit dem FIB wird zuerst bei hohen Ionenstrahlströmen von $7 \mathrm{nA}$ bis $20 \mathrm{nA}$ entlang der y-Achse in Abbildung 3.5 die grobe Form der Lamelle geschnitten. Es wird ein Block mit der Breite (x) von etwa $10 \mu \mathrm{m}$, einer Höhe (y) von etwa $20 \mu \mathrm{m}$ und einer Dicke (z) von etwa $5 \mu \mathrm{m}$ hergestellt. Ausgehend von diesem Block wird mit verschiedenen Ionenstrahlströmen die endgültige Geometrie (vgl. Abbildung 3.7a) geschnitten, sodass ein elektronentransparenter Bereich von etwa $2 \mu \mathrm{m}$ bis $3 \mu \mathrm{m}$ entsteht. Abschließend wird noch die initiale Kerbe hergestellt. Dazu wird die Lamelle parallel mit der z-Achse (vgl. Abbildung 3.5 zum Ionenstrahl ausgerichtet und mit dem geringsten zur Verfügung stehenden Ionenstrahl von $10 \mathrm{pA}$ eine Linie in -y-Richtung geschnitten. So können Krümmungsradien an der Spitze der Kerbe von wenigen $10 \mathrm{~nm}$ erreicht 


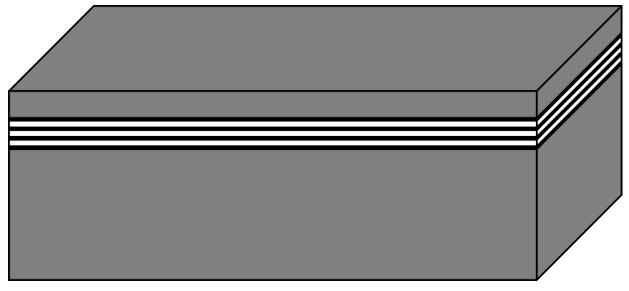

(a)

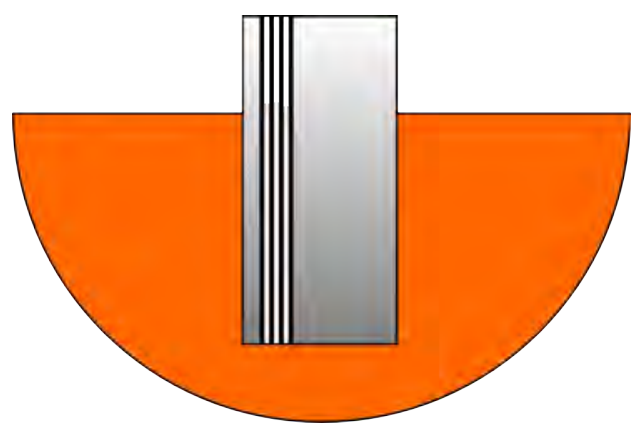

(c)

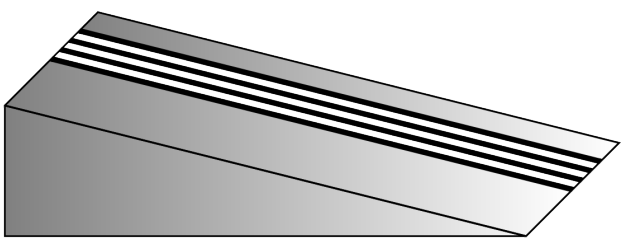

(b)

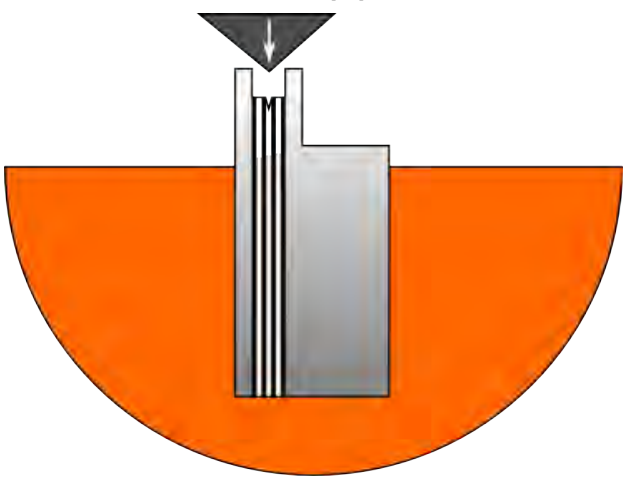

(d)

Abbildung 3.4: Skizzen zur Probenpräparation für Risse entlang der Grenzfläche:(a) mit der Wafersäge geschnittene dünne Streifen; (b)] nach dem mechanischen Schleifen als keilförmiger Stab; (c) aufgeklebt auf den Kupferhalter für den Einbau in den TEM-STM-Halter; (d) mit dem FIB aus der keilförmigen Probe präparierte Lamelle für die Bruchversuche

werden. Diese Krümmungsradien liegen damit in der gleichen Größenordnung wie die Einzelschichtdicken der verschiedenen Materialien in den Multilagen.

\section{Senkrechte Ausrichtung}

Die Präparationsschritte der Lamellen zur Rissausbildung senkrecht zur Grenzfläche der Multilagen sind schematisch in Abbildung 3.6 dargestellt. Die mit der Wafersäge geschnittenen Streifen werden entsprechend Abbildung 3.6b auf halbkreisförmige Kupferhalter aufgeklebt. Mit Hilfe des FIB können die Lamellen mit einem ähnlichen Prozess wie bereits beschrieben präpariert werden. Hier ist jedoch darauf zu achten, dass neben der eigentlichen Lamelle ausreichend viel Material entfernt wird, damit sich während des Bruchversuchs die Säulen frei nach außen verbiegen können (vgl. Abbildung 3.6c).

\subsubsection{Realisierung im TEM}

In den Abbildungen 3.7 und 3.8 sind jeweils Skizzen der Geometrie vor dem Kontakt zwischen Lamelle und Spitze (Abbildungen 3.7a und 3.8a) und während der Rissbewegung (Abbildungen 3.7b und 3.8b gezeigt. Diese Skizzen zeigen die hohe Flexibilität der gewählten Geometrie mit dem TEM-STM-Probenhalter. Ohne Schwierigkei- 


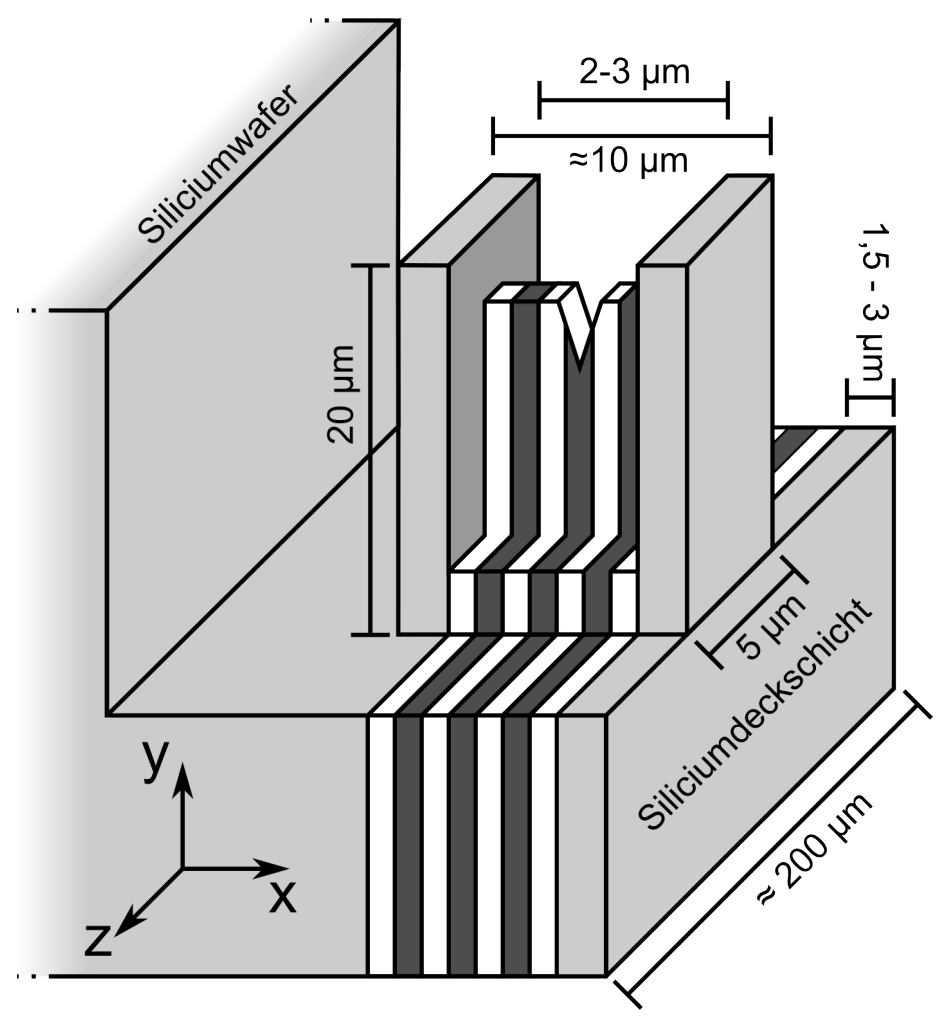

Abbildung 3.5: Skizze zur Probenpräparation im FIB

ten kann die Probenausrichtung um 90 relativ zur Annäherungsrichtung der Spitze gedreht werden, sodass sowohl die Rissbildung und -fortbewegung entlang der Grenzflächen zwischen $\mathrm{Ti}$ und $\mathrm{ZrO}_{2}$ als auch senkrecht zu diesen realisiert werden.

Die Geometrie, wie sie zum Beispiel in Abbildung 3.7a zu sehen ist, ermöglicht die Bildung eines Risses an der maschinell hergestellten Kerbe (vgl. Abschnitt 3.3.1) und dessen kontrollierte Bewegung durch die gesamte Probe. Die beiden Säulen am Rand der Multilage (grau dargestellt) werden durch die keilförmige Spitze kontinuierlich weiter nach außen gebogen. Diese Verbiegung ist zum Großteil elastisch, da nach dem Zurückziehen der STM-Spitze sich die Säulen ebenfalls zurück in die Anfangsposition biegen. Lediglich an den direkten Kontaktpunkten zwischen Spitze und Säulen kann eine geringfügige plastische Verformung der Säulen beobachtet werden. Die Säulen dienen somit als Biegebalken, deren untere Enden fixiert sind. Durch diese Verbiegung wird eine Zugspannung auf die Multilagen zwischen den Säulen ausgeübt. Durch die Spannung öffnet sich die vordefinierte Kerbe und an der Spitze dieser Kerbe bildet sich ein Riss. Um diese Spannung homogen zu erzeugen, werden beide Säulen möglichst gleich groß hergestellt. Sobald die STM-Spitze nicht weiter zwischen die beiden Säulen getrieben wird, stoppt die Rissbewegung. Entsprechend kann durch erneute Bewegung der STM-Spitze der Riss zu einer Bewegung stimuliert werden.

Für die Geometrie der Rissbewegung senkrecht zu den Grenzflächen zwischen Ti und $\mathrm{ZrO}_{2}$ gelten die oben angestellten Überlegungen, sodass auch in dieser Geometrie die 


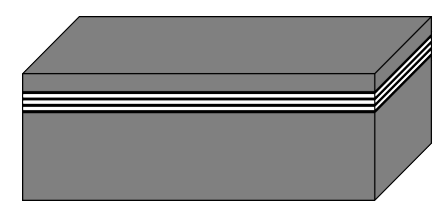

(a)

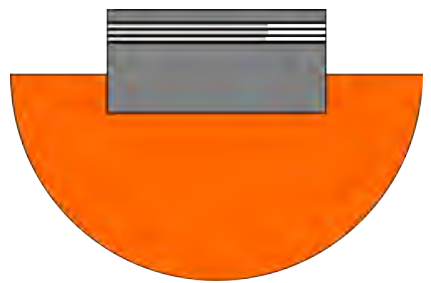

(b)

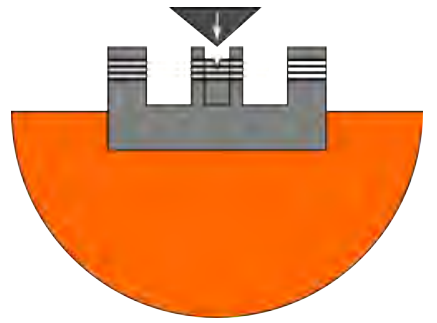

(c)

Abbildung 3.6: Skizzen zur Probenpräparation für Risse senkrecht zu der Grenzfläche:(a) mit der Wafersäge geschnittene dünne Streifen; (b) entsprechend der Orientierung der Multilagen aufgeklebt auf Kupferhalter; (c) mit dem FIB aus der keilförmigen Probe präparierte Lamelle für die Bruchversuche

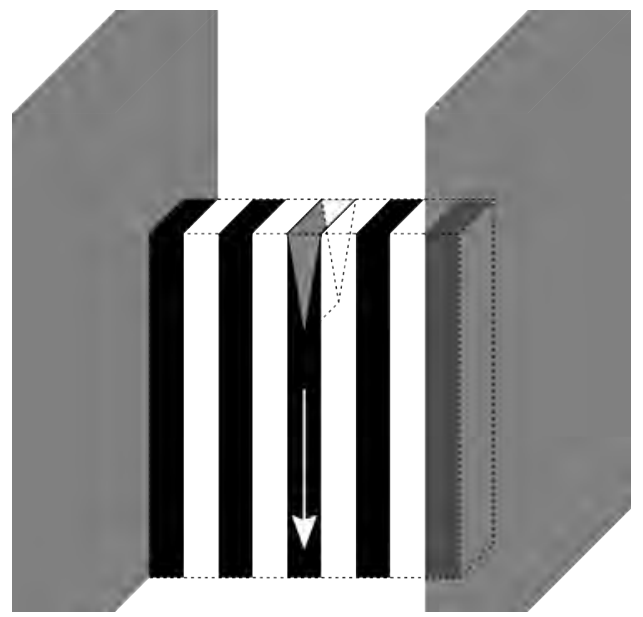

(a)

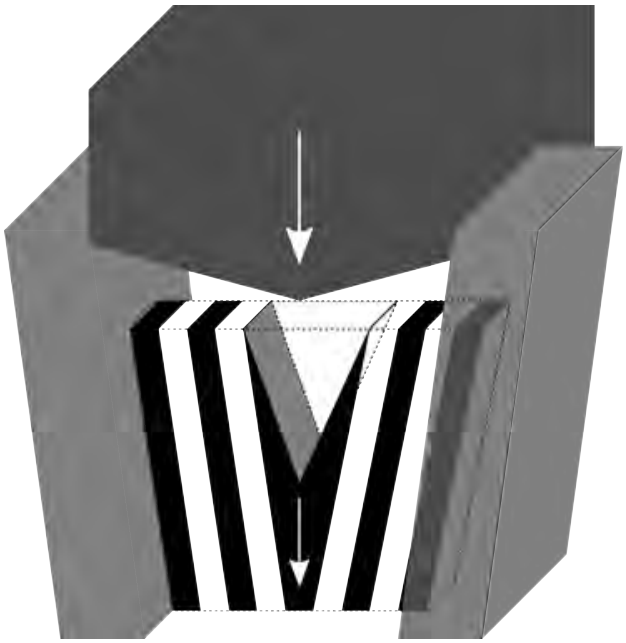

(b)

Abbildung 3.7: Skizzen zum Bruchversuch entlang der Grenzfläche: (a) Geometrie vor dem Bruchversuch;(b) Lamelle im Kontakt mit der Spitze

Rissbildung und -bewegung von der vordefinierten Kerbe aus analysiert werden kann. Die sehr kleinen Bewegungsschritte des TEM-STM-Probenhalters ermöglichen in Verbindung mit der gewählten Geometrie eine kontrollierte und geringe Rissgeschwindigkeit, sodass die Prozesse der Rissbildung und -bewegung direkt beobachtet werden können. Zusätzlich verfügt der TEM-STM-Probenhalter über eine hinreichend hohe Stabilität nach der Bewegung der STM-Spitze, die es erlaubt, die neu entstandenen Oberflächen entlang der Bewegungsrichtung des Risses sowohl mit analytischen Methoden als auch mit hochauflösender Mikroskopie zu charakterisieren. 


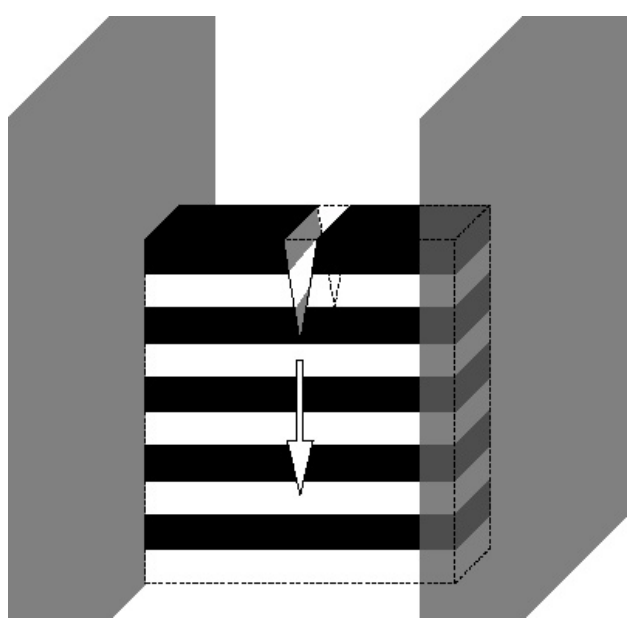

(a)

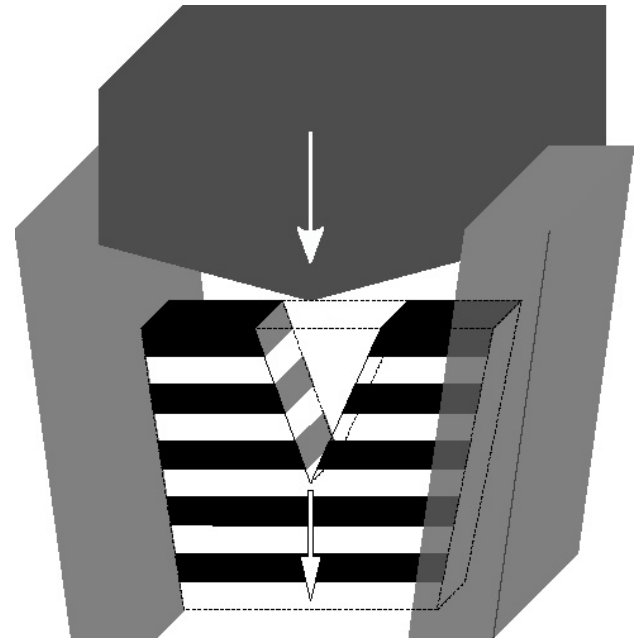

(b)

Abbildung 3.8: Skizzen zum Bruchversuch senkrecht zu den Grenzflächen:(a) Geometrie vor dem Bruchversuch;(b) Lamelle im Kontakt mit der Spitze; Die Multilagen sind nur auf der rechten Säule aus Gründen der Übersichtlichkeit dargestellt.

\subsection{Auswertung der Bruchversuche}

\subsubsection{Energiefreisetzungsrate}

Basierend auf der Annahme der elastisch-plastischen Bruchmechanik werden die Bruchversuche ausgewertet. Aus den aufgenommenen Bildserien während der Rissbildung und -bewegung können quantitative Ergebnisse zusätzlich zur rein qualitativen Beschreibung des Rissprozesses gewonnen werden. Zum einen wird die Größe der plastischen Zone abgeschätzt. Dieses geschieht anhand der auftretenden Kontrastwechsel in den TEM-Aufnahmen während der Verformung. Diese Kontrastwechsel spiegeln plastische Verformung wider, sodass sie als Maß für die Größe oder den Radius der plastischen Zonen genutzt werden können.

Zum anderen kann die Rissspitzenaufweitung kontinuierlich für die jeweilige Bildserie ausgemessen und analysiert werden. Die Rissspitzenaufweitung ermöglicht entsprechend Gleichung (2.7) die Berechnung der Energiefreisetzungsrate J. Die Ausmessung der Rissspitzenaufweitung erfolgt mittels eines MATLAB-Skripts, dessen Funktionsweise nachfolgend schematisch erklärt wird. Zu Beginn werden alle Bilder einer Bildserie um eine Verschiebung des Bildes durch den Bruchversuch korrigiert, sodass die Spitze der ursprünglichen Kerbe an der Position der Kerbe im ersten Bild der Serie ausgerichtet ist (vgl. Abbildung 3.9a). Die Position der Spitze der Kerbe wird im ersten Bild manuell festgelegt. Mit Hilfe der skaleninvarianten Merkmalstransformation (engl. scale-invariant feature transform, SIFT) werden markante Merkmale der Bilder erkannt [Low04. Über den Vergleich dieser Merkmale in verschiedenen Bildern der 


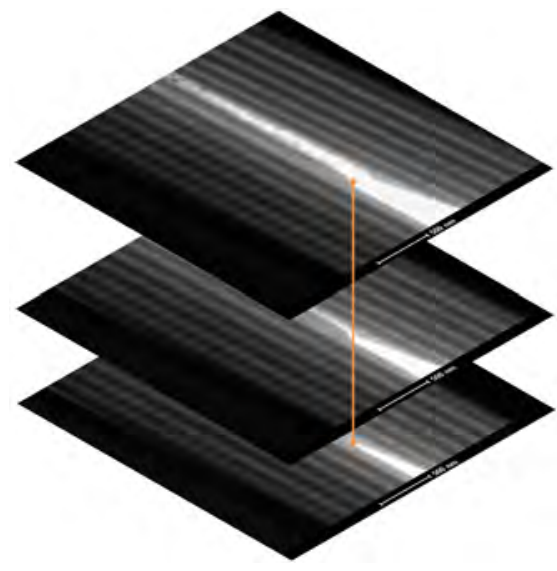

(a)

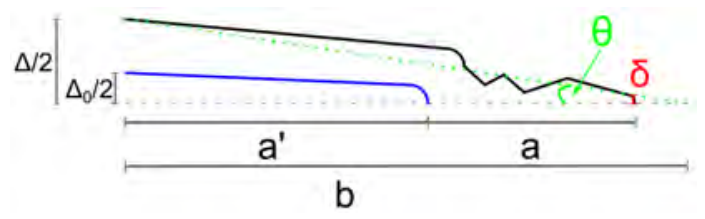

(b)

Abbildung 3.9: Skizze zur Bestimmung der Rissspitzenaufweitung: (a) schematisch dargestellt die Ausrichtung der Einzelbilder entlang der eingezeichneten Linie, damit die Spitze der Kerbe in allen Bildern an der gleichen Position im Bild ist; (b) die aus den Bildern bestimmten Größen zur Berechnung der Rissspitzenaufweitung, nur eine Hälfte des Risses gezeigt

Bildserie können lineare Verschiebungen der Merkmale berechnet werden. Diese Berechnung erfolgt mit einer Erweiterung des Bildbearbeitungsprogramms ImageJ [Sch12b]. Im MATLAB-Skript werden die Verschiebungen auf die Bilder angewendet, sodass in der korrigierten Bildserie die Kerbe ausgerichtet ist. Die Bilder werden dabei nicht verzerrt oder zugeschnitten, es geht keine Information verloren.

An den korrigierten Bildserien kann die Rissspitzenaufweitung $\delta$ (rot in Abb. 3.9b) ausgemessen werden. In jedem Einzelbild einer Serie werden die verschiedenen Längen und der Öffnungswinkel wie in Abbildung $3.9 \mathrm{~b}$ dargestellt bestimmt. $\Delta_{0}$ ist hier die Breite der Kerbe (blaue Linie) vor dem Bruchversuch. Die schwarze Linie in Abbildung $3.9 \mathrm{~b}$ stellt die sich öffnende Kerbe incl. Risswachstum dar. Durch die Verformung weitet sich die ursprüngliche Kerbe und der Riss wird gebildet bzw. wächst weiter. Die Öffnungsweite ist jeweils $\Delta$. Aus der Differenz der Öffnungsweite $\Delta$ in den fortlaufenden Bildern und der anfänglichen Öffnungsweite $\Delta_{0}$ lässt sich die tatsächliche Öffnung der Kerbe bzw. des Risses berechnen. Über die Abstände von dem Bestimmungsort von $\Delta$ sowie $\Delta_{0}$ zur Spitze der Kerbe $a^{\prime}$ und zum Schnittpunkt der Flanken $b$ kann die Rissspitzenaufweitung $\delta$ entsprechend Gleichung (3.2) berechnet werden.

$$
\delta=\frac{\left(b-a^{\prime}-a\right)\left(\Delta-\Delta_{0}\right)}{b}
$$

Über Gleichung 2.7) wird somit die Energiefreisetzungsrate für die in-situ Bruchversuche anhand der aufgenommenen Bildserien bestimmt. Da die Lamellen alle extrem dünn sind, ist die Annahme eines ebenen Spannungszustandes, $m=1$ gerechtfertigt. Die Fließspannung $\sigma_{y s}$ der verschiedenen Proben ist durch die mechanische Charakterisierung in [Kno12] bekannt und wird entsprechend Tabelle 3.2 verwendet. 


\begin{tabular}{lccc} 
& \multicolumn{2}{c}{ Komposit } & $\mathrm{Ti}$ \\
$\mathrm{Ti} / \mathrm{ZrO}_{2}$-Probe & $\sigma_{y s}[\mathrm{GPa}]$ & $E[\mathrm{GPa}]$ & $\sigma_{y s}[\mathrm{GPa}]$ \\
\hline \hline $100 / 100$ & 4,7 & 144,5 & 2,4 \\
$50 / 100$ & 5,4 & 142,8 & 1,9 \\
$10 / 100$ & 6,5 & 164,3 & 2,865 \\
\hline $\mathrm{Ti}$ & 1,8 & 150,8 & \\
$\mathrm{ZrO}_{2}$ & 6,8 & 188,3 &
\end{tabular}

Tabelle 3.2: Fließspannung und Elastizitätsmodul für $\mathrm{Ti}^{2} / \mathrm{ZrO}_{2}-$ Proben. Die aufgelisteten Werte stammen sowohl aus Mikrodruckversuchen als auch Berkovich-Indentation der entsprechenden Multilagen. Die Fließspannungen des Titans sind anhand einer Mischungsregel aus den Kompositdaten extrahiert worden. Die Einzelkomponentendaten sind jeweils an ca. $3 \mu \mathrm{m}$ dicken Schichten gemessen worden. Alle Einzelheiten sind [Kno12] zu entnehmen.

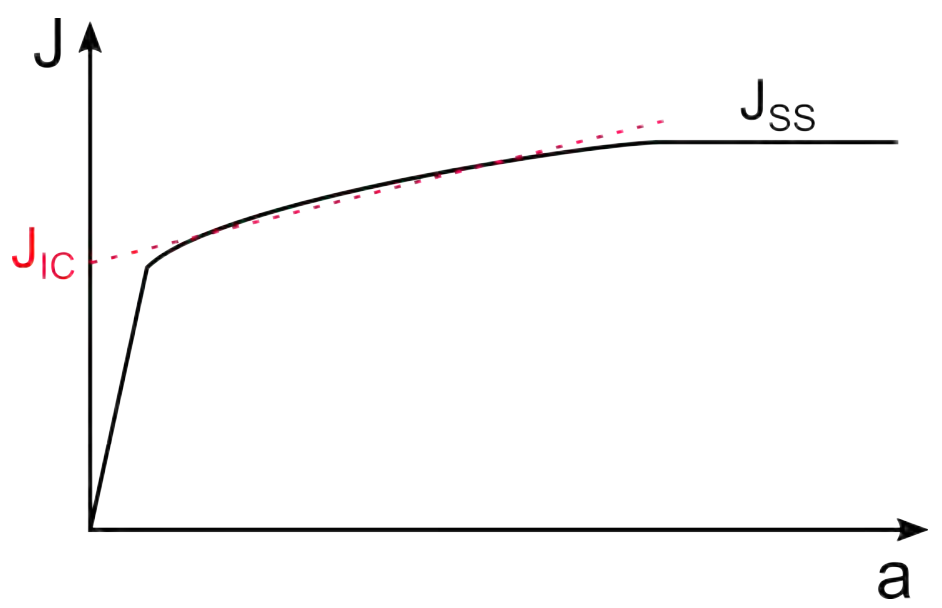

Abbildung 3.10: Schematische Energiefreisetzungsrate vs. Risslänge-Kurve und linearer Fit zur Bestimmung der Energiefreisetzungsrate bei der Rissbildung, $J_{S S}$ entspricht der Energiefreisetzungsrate für Gleichgewichtsrisswachstum

Aus der Auftragung der Energiefreisetzungsrate $J_{I}$ gegen die Risslänge a (vgl. Abbildung 3.10) wird der kritische Wert $J_{I Q}$ bestimmt. In dieser Arbeit wird der kritische Wert für $J$ bei der Rissbildung anhand des Ordinatenabschnitts einer linearen Anpassung an die experimentell gemessenen Daten bestimmt. Über die folgende Gleichung (3.3) werden aus $J_{I Q}$ mit dem Elastizitätsmodul $E$ (Tabelle 3.2) kritische Bruchzähigkeiten $K_{I Q}$ bei der Rissbildung errechnet.

$$
K_{I Q}=\sqrt{J_{I Q} E}
$$

Die gewählte Geometrie der Bruchversuche ist bereits auf größeren Längenskalen in ähnlicher Weise genutzt worden, um Bruchzähigkeiten zu messen. Eine theoretische Analyse der Bruchzähigkeiten im Vergleich zu ASTM-standardisierten Bruchgeometrien hat gezeigt, dass die gewählte Bruchgeometrie Bruchzähigkeit akkurat messen 


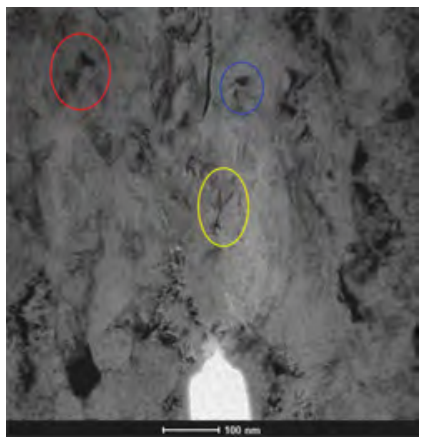

(a) 1

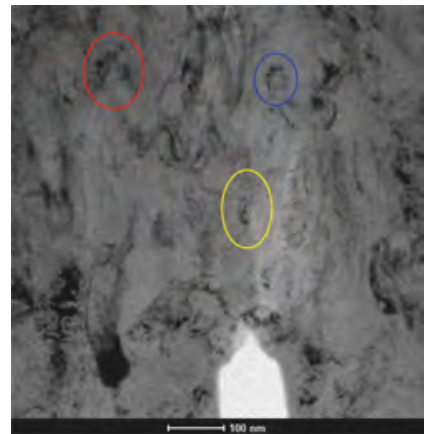

(b) 300

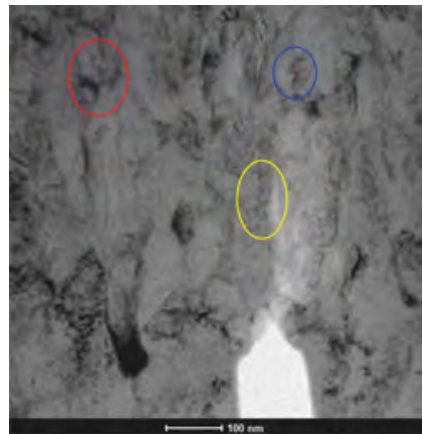

(c) 500

Abbildung 3.11: Bestimmung der Größe der plastischen Zone anhand verschiedener Einzelbilder einer Bildserie, hier exemplarisch drei Bilder einer Bildserie eines Bruchversuchs einer Ti-Einzelschicht

kann Gui96. In in-situ Biegebalkenversuchen in einem SEM konnte nachgewiesen werden, dass die Auswertung der Bruchversuche über die Energiefreisetzungsrate sowie die CTOD funktioniert und sinnvolle kritische Bruchzähigkeiten für NiAl gemessen werden konnten [Ast14].

\subsubsection{Plastische Zone}

In den Abbildungen 3.11a bis 3.11c sind drei Einzelbilder eines Verformungsschrittes des Bruchversuchs einer Ti-Einzelschicht gezeigt (vgl. Abschnitt 4.3.1). An den drei markierten Stellen in den gezeigten Abbildungen können im Verlauf der Bildserie mehrere Kontrastwechsel beobachtet werden. Solche Kontrastwechsel werden im Allgemeinen mit der Aktivität von Versetzungen interpretiert. Es kann jedoch nicht ausgeschlossen werden, dass die Kontrastveränderungen durch die Rotation von einzelnen Körnern entstehen, wenn die Orientierungen der Körner während der Verformung zufällig die Bragg-Bedingung erfüllen oder nicht. Eine solche Rotation der Körner kann zum Beispiel in freistehenden Gold-Schichten (Schichtdicken zwischen $100 \mathrm{~nm}$ bis $300 \mathrm{~nm}$ ) beobachtet werden [Mer14].

Zusätzlich zu der Information, dass innerhalb der Proben Versetzungsaktivität vorhanden ist, kann über die Abstände der Kontrastwechsel von der Spitze der Kerbe oder der Rissspitze die Größe der plastischen Zone in der Ausbreitungsrichtung des Risses abgeschätzt werden. Die Abschätzung erfolgt für jede geeignete Bildserie separat. Es werden weiterhin mehrere Kontrastwechsel analysiert, wie in Abbildung 3.11a bis 3.11c dargestellt. Die im Verlauf dieser Arbeit angegebenen plastischen Zonen verstehen sich als Mittelwert mehrerer ausgewerteter Kontrastwechsel.

Durch die gewählte Vergrößerung im TEM ist der tatsächliche Bildausschnitt festgelegt, sodass alle angegebenen plastischen Zonen als untere Grenze der Größe der plastischen Zone interpretiert werden müssen. Ob außerhalb des Bildausschnitts Versetzungsakti- 


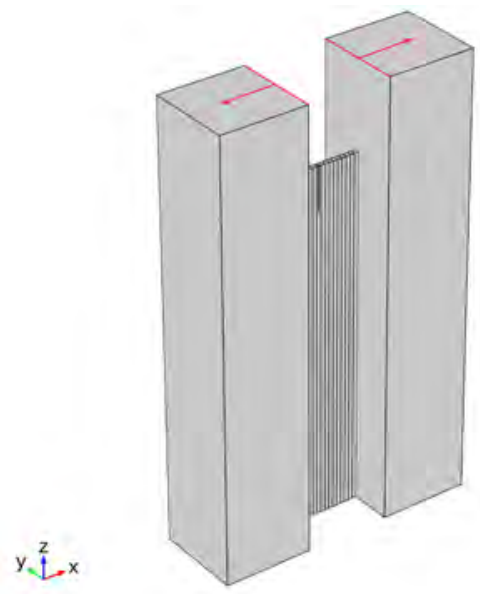

(a)

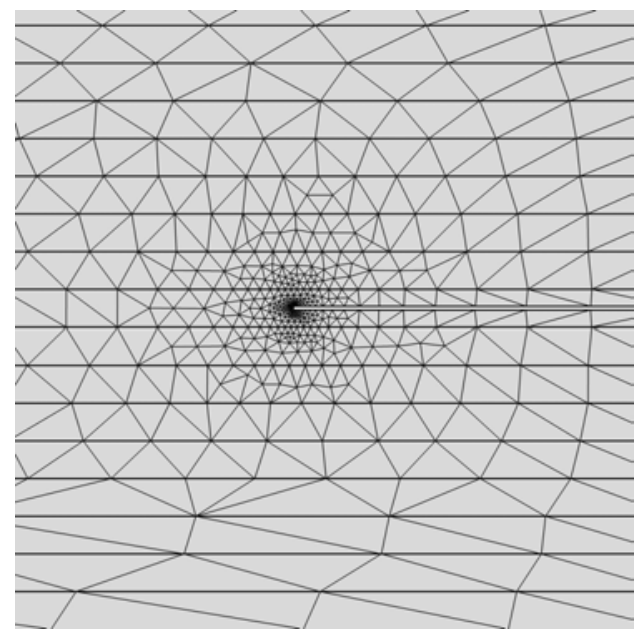

(b)

Abbildung 3.12: COMSOL Simulationen: (a) Skizze der Geometrie, wie sie in COMSOL realisiert wird, Dimensionen siehe Text; (b) Detailaufnahme der Vernetzung in der Nähe der Spitze der Kerbe

vität stattfindet, kann nicht festgestellt werden, sodass die Größe der plastischen Zone nur eine Abschätzung ist.

\subsection{FEM-Simulationen}

\subsubsection{COMSOL Multiphysics}

Die verschiedenen Geometrien der Bruchversuche werden bezüglich der Verteilung der Spannungen in den Lamellen mit der Software COMSOL Multiphysics (Version 4.2a; COMSOL Multiphysics GmbH, Göttingen, Deutschland) simuliert. Die gestellten Probleme werden mit Hilfe von partiellen Differentialgleichungen beschrieben, die mit der Finite-Elemente-Methode (FEM) gelöst werden. Das numerische Lösungsverfahren beinhaltet hier zuerst die Unterteilung des Objekts in eine endliche Anzahl von Elementen, für welche die Lösung des ursprünglich gestellten Problems anhand gegebener Randbedingungen approximiert wird. In allen durchgeführten Simulationen werden die Geometrien ausschließlich elastisch verformt.

Abbildung 3.12 zeigt die anfängliche Geometrie der Lamelle der Bruchversuche. Die tatsächlichen Dimensionen der Lamellen sind weitestgehend übernommen worden. Die Si-Säulen haben eine Abmessung von $2 \mu \mathrm{m} \times 2 \mu \mathrm{m} \times 10 \mu \mathrm{m}$ und die einzelnen Schichten $0,1 \mu \mathrm{m} \times 0,1 \mu \mathrm{m} \times 8 \mu \mathrm{m}$. Die Kerbe ist $1 \mu \mathrm{m}$ lang und $10 \mathrm{~nm}$ breit mit einer symmetrisch abgerundeten Spitze.

Die Randbedingungen sind wie folgt gewählt. Die gesamte Lamelle ist am unteren Ende mit festen Randbedingungen in der xy-Ebene fixiert. Das Verbiegen der Si-Säulen durch die keilförmige STM-Spitze wird durch vorgegebene Verschiebungen 
der rot markierten Kanten der Säulen simuliert (vgl. Abbildung 3.12a). Alle anderen Bewegungen sind frei möglich.

Die Unterteilung der Lamelle erfolgt mit tetragonalen Elementen variabler Größe, sodass die kritische Stelle an der Spitze der Kerbe hinreichend präzise unterteilt werden kann. In Abbildung 3.12b ist die gewählte Vernetzung an der Kerbe dargestellt.

Die verschiedenen Materialien der Bruchversuche werden durch die Angabe der gemessenen Elastizitätsmoduln in die Simulationen eingebunden (vgl. Tabelle 3.2). Der Elastizitätsmodul für Si wird als $170 \mathrm{GPa}$ angenommen. 


\section{Experimentelle Ergebnisse}

In den folgenden Abschnitten werden die gewonnenen experimentellen Daten dargestellt. Der Anfangszustand der verschiedenen Proben wird mit hochauflösender Transmissionselektronenmikroskopie (engl. high resolution transmission electron microscopy, HRTEM), Elektronenenergieverlustspektroskopie (engl. electron energy loss spectroscopy, EELS) sowie energiegefilterter Transmissionselektronenmikroskopie (engl. energy filtered transmission electron microscopy, EFTEM) für alle verschiedenen Schichtdicken mit besonderem Augenmerk auf die Grenzflächen charakterisiert. Des Weiteren werden einige Kontrollexperimente an Einzelschichten der beiden Komponenten und Bruchversuche ohne initiale Kerbe zur Rissbildung beschrieben. Anschließend werden die experimentellen Ergebnisse der in-situ Bruchversuche sowohl entlang als auch senkrecht zu den Grenzflächen der Multilagen dargestellt und analysiert. Der Einfluss einer Gasatmosphäre auf das Bruchverhalten der Multilagen wird abschließend bearbeitet.

\subsection{Charakterisierung der Multilagen}

Alle verwendeten Schichtdickenkombinationen der Multilagen werden vor den eigentlichen Bruchversuchen mittels verschiedener bildgebender sowie spektroskopischer Methoden eingehend charakterisiert. Diese Charakterisierung erfolgt an speziell präparierten Lamellen, nicht an den späteren Proben für die Bruchversuche. In Abbildung 4.1 sind Übersichtsaufnahmen der drei verschiedenen verwendeten Proben gezeigt. Die $\mathrm{ZrO}_{2}$-Schichtdicke ist in allen Proben konstant mit $d_{\mathrm{ZrO}_{2}} \approx 75 \mathrm{~nm}$. Die Diskrepanz zwischen geplanter Schichtdicke von $100 \mathrm{~nm}$ und der tatsächlich gemessenen Dicke ergibt sich aus der Tatsache, dass die hier genutzten Proben vom Rand eines $1 \mathrm{~cm} \mathrm{x}$ $2 \mathrm{~cm}$ großen Silicium-Substrates entnommen werden. Der Schichtdickengradient, bedingt durch die gepulste Laserdeposition (engl. pulsed laser deposition, PLD) und gekoppelt an die Größe des Substrates, führt dazu, dass alle gemessenen Schichtdicken 


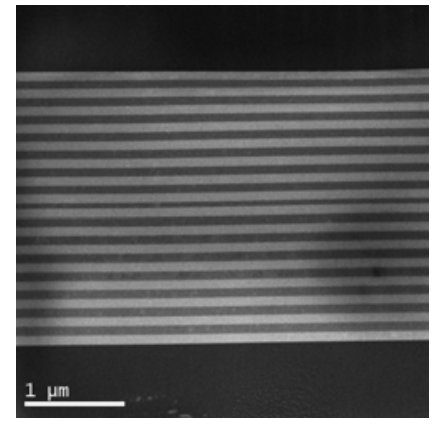

(a) HAADF Ti100/ZrO 2100

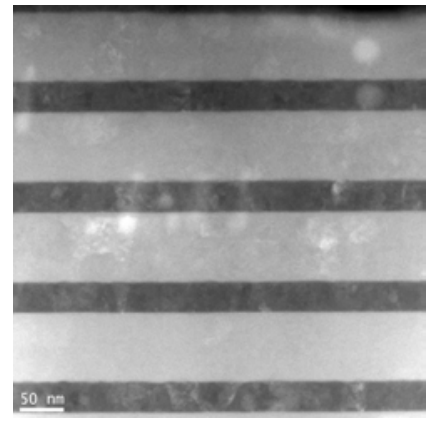

(b) ADF Ti50/ZrO 100

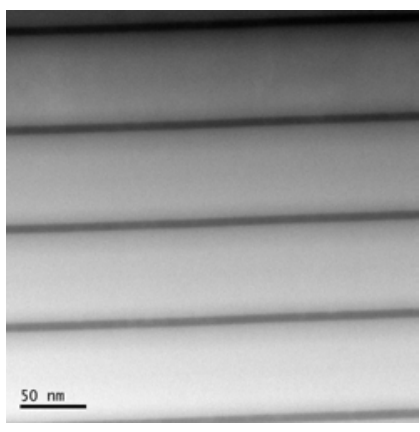

(c) HAADF Ti10/ZrO 2100

Abbildung 4.1: STEM-Dunkelfeldübersichtsaufnahmen der verwendeten Proben bei verschiedenen Vergrößerungen. Die Ti-Schichten sind dunkel, das Substrat ist unten

signifikant geringer sind als die geplanten Dicken bei der Herstellung. Die Nomenklatur bezieht sich weitergehend auf die geplanten Schichtdicken. In Tabelle 4.1 sind die gemessenen Schichtdicken der drei Proben dargestellt. Im Zentrum des Substrates werden die Schichtdicken sehr gut erzielt (vgl. Tabelle 4.2 in [Kno12]).

In den Übersichtsaufnahmen zeigt sich gut, dass die Proben auf verschiedenen Längenskalen sehr gewachsen sind. Alle Grenzflächen sind glatt mit sehr geringen Rauigkeiten, die anhand von hochaufgelösten Aufnahmen abgeschätzt werden können (vgl. zum Beispiel Abbildung 4.4). Im Fall der Ti100/ZrO 2 100-Probe ist eindeutig eine dünnere Ti-Schicht in der Mitte der Multilage zu erkennen. Die Abweichung in der Schichtdicke dieser einzelnen Schicht lässt sich nicht mehr nachvollziehen. Da jedoch alle anderen Schichten gleich dick für die jeweilige Multilage sind, ist von einer einmaligen Abweichung während des Herstellungsprozesses auszugehen.

In der höher vergrößerten Aufnahme der Ti50/ZrO 2 100-Probe (Abbildung 4.1b) sind Kontrastveränderungen in beiden Schichten zu erkennen. Im Fall der Ti-Schichten sind diese mit Orientierungskontrasten der verschiedenen Körner zu benennen. Röntgenund TEM Untersuchungen an diesem Schichtsystem haben gezeigt, dass die Korngröße in der Schichtebene jeweils ca. die Hälfte der Schichtdicke beträgt Kno12, Lie07, Lie12. Die hier gezeigten Dunkelfeldaufnahmen (engl. dark field micrograph, DF) in Abbildungen 4.2 und 4.3 bestätigen, dass die Korngröße jeweils etwa der halben Schichtdicke entspricht. Senkrecht zu den Grenzflächen wächst das Ti weitestgehend kollumnar auf (vgl. TEM-Untersuchungen in Kno12). Das kollumnare Wachstum kann durch die exemplarischen Dunkelfeldaufnahmen in Abbildung 4.2 eindeutig nachgewiesen werden. Lediglich bei den dicken Ti-Schichten mit $100 \mathrm{~nm}$ Schichtdicke zeigt sich ein verändertes Wachstum. Hier werden zu Beginn der Deposition kleine Körner direkt an der Grenzfläche zur vorherigen Schicht gebildet, auf denen mit steigender Schichtdicke die Körner kollumnar aufwachsen wie auch bei den dünneren Schichten. Bevorzugt an den Grenzflächen zur vorangegangenen $\mathrm{ZrO}_{2}$-Schicht können hier in Dunkelfeldaufnahmen Körner nachgewiesen werden, die nicht kollumnar über die gesamte Schichtdicke ge- 


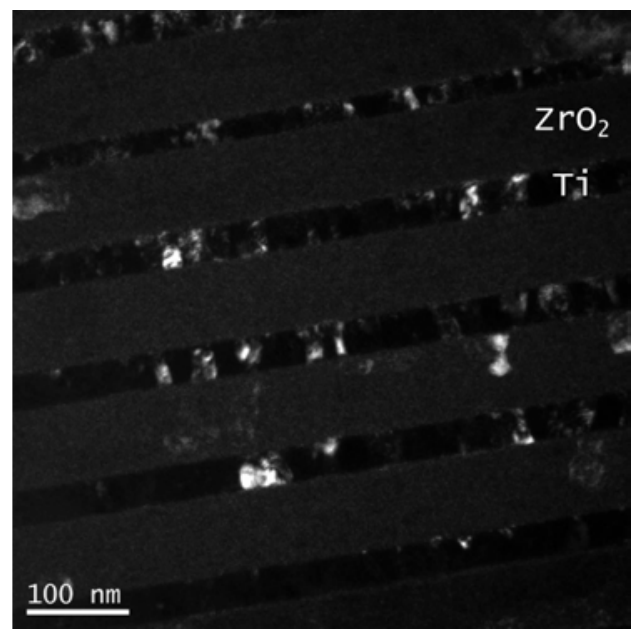

(a) $\mathrm{Ti} 50 / \mathrm{ZrO}_{2} 100$

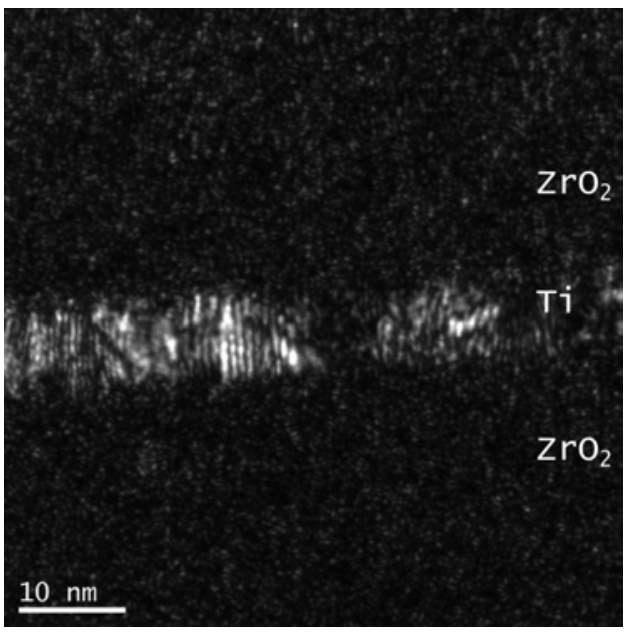

(b) $\mathrm{Ti} 10 / \mathrm{ZrO}_{2} 100$

Abbildung 4.2: Dunkelfeldaufnahmen der Proben $\mathrm{Ti} 50 / \mathrm{ZrO}_{2} 100$ und $\mathrm{Ti} 10 / \mathrm{ZrO}_{2} 100$, Substrat jeweils oben

\begin{tabular}{lccc}
$\begin{array}{l}\mathrm{Ti} / \mathrm{ZrO}_{2} \\
\text { Probe }\end{array}$ & $\begin{array}{c}t_{\mathrm{Ti}} \\
{[\mathrm{nm}]}\end{array}$ & $\begin{array}{c}t_{\mathrm{ZrO}_{2}} \\
{[\mathrm{~nm}]}\end{array}$ & $\begin{array}{c}\text { \# der } \\
\text { Doppelschichten }\end{array}$ \\
\hline \hline $10 / 100$ & 7 & 70 & 27 \\
$50 / 100$ & 35 & 80 & 20 \\
$100 / 100$ & 78 & 86 & 15
\end{tabular}

Tabelle 4.1: Gemessene Einzelschichtdicken $t_{\mathrm{Ti}}$ und $t_{\mathrm{ZrO}_{2}}$ und Anzahl der Doppelschichten für die untersuchten $\mathrm{Ti}_{2} / \mathrm{ZrO}_{2}$-Proben

wachsen sind (vgl. Abbildung 4.3b). Jedoch gibt es auch kollumnares Wachstum, wie bereits bei den dünneren Ti-Schichten.

Des Weiteren ist das Wachstum des Ti in der Schichtebene parallel zu den Grenzflächen abhängig von der Schichtdicke. Für Schichtdicken zwischen $50 \mathrm{~nm}$ bis $100 \mathrm{~nm}$ wird eine starke (1 $0 \overline{1} 0$ )-Textur ausgeprägt, wobei hingegen bei $10 \mathrm{~nm}$ Schichtdicke eine (0 000 2)-Textur beobachtet wird. Die veröffentlichte Literatur bezüglich des Wachstums von Ti-Schichten zeigt auch beide hier gefundenen Texturen. Magnetron-gesputterte

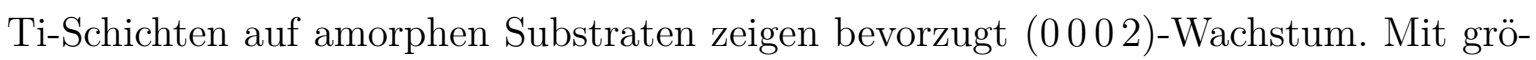
ßerer Energie der Ti-Partikel ist ein Wechsel zu $\langle 10 \overline{1} 0\rangle$-Wachstum beobachtet worden [Son07]. Die Partikelenergien in der PLD liegen im Bereich von ca. $100 \mathrm{keV}$ und sind damit deutlich größer im Vergleich zu Magnetronsputteranlagen, sodass ein bevorzugtes Wachstum in $\langle 10 \overline{1} 0\rangle$-Richtung für PLD-Ti-Schichten zu erwarten ist.

In Abbildung 4.4 sind hochaufösende TEM-Aufnahmen der beiden verschiedenen Grenzflächen einer Ti100/ZrO 2 100-Probe gezeigt. Es sind die Grenzflächen der jeweiligen Art abgebildet, die zuletzt hergestellt worden sind. Zum Beispiel im Fall der Ti100/ZrO 2 100-Probe die letzte der 15 Doppelschichten. Die Grenzflächen zwischen den amorphen und kristallinen Schichten sind eindeutig erkennbar. Die Grenzflächen- 


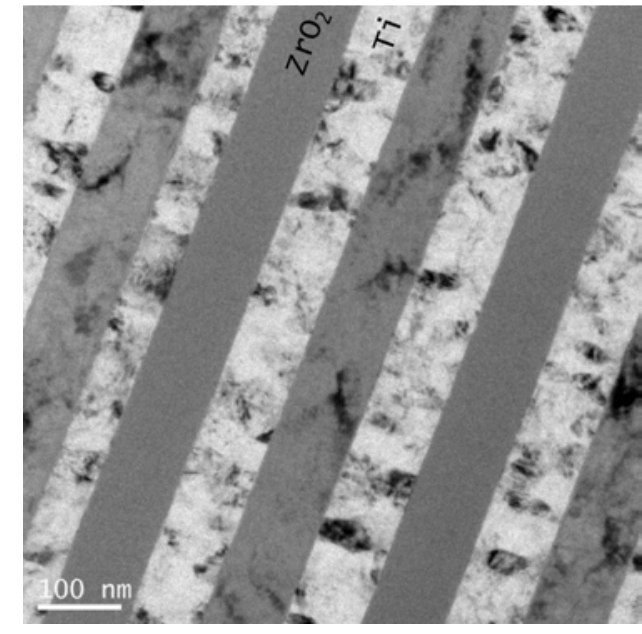

(a)

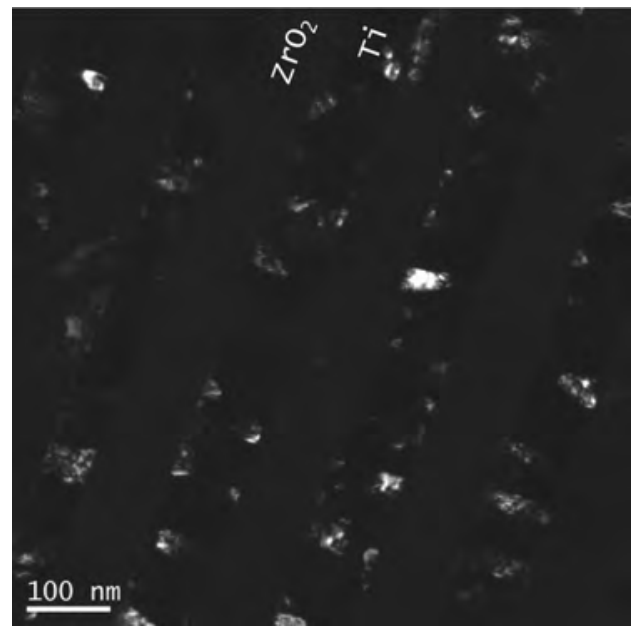

(b)

Abbildung 4.3: Hellfeld- und Dunkelfeldaufnahmen Probe Ti100/ $\mathrm{ZrO}_{2} 100$; Substrat ist links

rauigkeit in diesen Querschnittsaufnahmen beträgt weniger als $1 \mathrm{~nm}$, wobei die Grenzfläche im Falle der Deposition von Ti auf $\mathrm{ZrO}_{2}$ etwas glatter bzw. schärfer ist. Speziell im Fall der Ti50/ZrO 2 100-Probe (vgl. Abbildung 4.5a) ist die leichte Asymmetrie der Grenzflächen zu sehen. Die Erhebungen an der Grenzfläche zwischen $\mathrm{ZrO}_{2}$ und Ti lässt auf die einzelnen, kollumnar gewachsenen Körner im Ti zurück schließen. Durch die polykristalline Struktur des $\mathrm{Ti}$ ist diese $\mathrm{ZrO}_{2} /$ Ti-Grenzfläche weniger glatt im Vergleich zur $\mathrm{Ti} / \mathrm{ZrO}_{2}$-Grenzfläche. Diese Asymmetrie lässt sich in Verbindung mit den glättenden Eigenschaften des PLD-ZrO 2 bei der Schichtherstellung erklären Röd08.

Die Grenzflächen bei den Proben mit dünneren Ti-Schichten sind gut vergleichbar in den hochaufgelösten TEM-Aufnahmen (vgl. Abbildung 4.5). Die Grenzflächenrauigkeiten für die beiden dünneren Ti-Schichten liegen auch im Bereich von $1 \mathrm{~nm}$, wie schon bei den dickeren Schichten, sodass die Rauigkeit unabhängig von der Schichtdicke ist. Die gezeigten HRTEM-Aufnahmen verstehen sich als repräsentative Darstellung der Grenzflächengegebenheiten der verschiedenen Proben. Weiterhin zeigen andere Grenzflächen näher zum Substrat eine vergleichbare Rauigkeit auch über größere Längen als in den HRTEM-Aufnahmen gezeigt.

In Abbildung 4.4b sind in der amorphen $\mathrm{ZrO}_{2}$-Schicht kleine kristalline Bereiche (rote Pfeile) zu sehen. Diese Kristallisation des amorphen Materials ist eindeutig mit dem Beschuss durch Ga-Ionen im FIB in Verbindung zu bringen, sodass an verschiedenen Stellen in den amorphen Schichten kristalline Strukturen gefunden werden können. In den bereits beobachteten Kontrasten im $\mathrm{ZrO}_{2}$ in Abbildung 4.1b zeigt das $\mathrm{ZrO}_{2}$ ebenfalls kristalline Strukturen. Daraus folgt, dass in Bereichen veränderten Kontrastes im $\mathrm{ZrO}_{2}$ mit ionenstrahlinduzierter Kristallisation $\mathrm{zu}$ rechnen ist. Aus der Literatur ist dieser Effekt bereits in amorphen $\mathrm{ZrO}_{2}$-Schichten beobachtet worden [Hei11a, Lia09]. Eine weitere Analyse der Kristallisation der amorphen Schichten durch den Ionenstrahl 


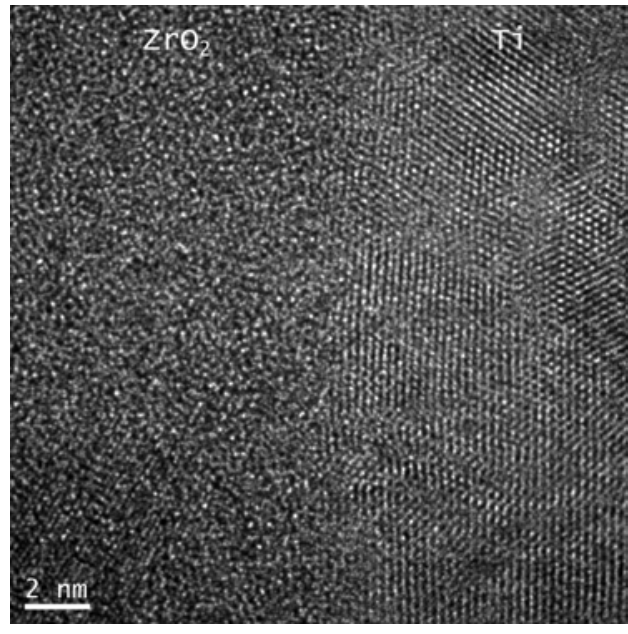

(a) HRTEM $\mathrm{ZrO}_{2}$ auf Ti

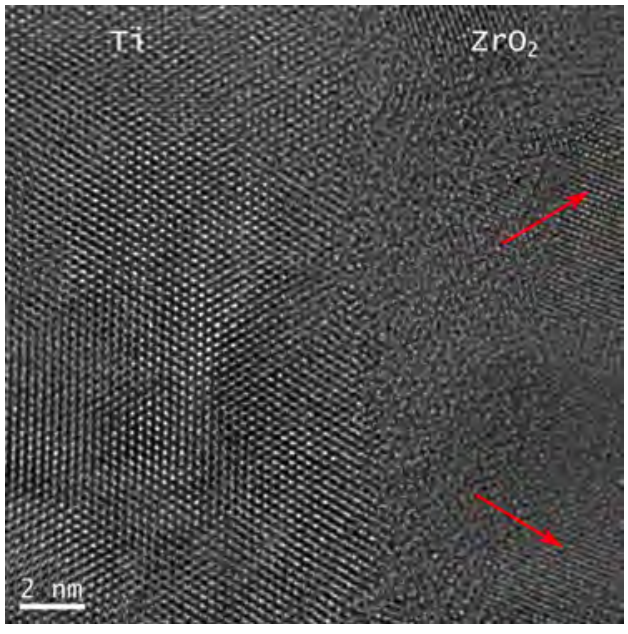

(b) HRTEM Ti auf $\mathrm{ZrO}_{2}$

Abbildung 4.4: HRTEM-Aufnahmen Probe Ti100/ZrO ${ }_{2} 100$, Substrat liegt jeweils rechts: (a) Grenzfläche zwischen der abschließenden $\mathrm{ZrO}_{2}$-Deckschicht und der zuletzt deponierten Ti-Schicht; (b) Grenzfläche zwischen der zuletzt deponierten Ti-Schicht und der vorletzten $\mathrm{ZrO}_{2}$-Schicht, kristalline Bereiche in den $\mathrm{ZrO}_{2}$-Schichten sind markiert

im FIB ist im Anhang C dieser Arbeit zu finden.

\subsubsection{EELS-Analyse der Grenzflächen}

Zusätzlich zu der HRTEM-Analyse werden die Grenzflächen der verwendeten Proben mit räumlich hochaufgelösten EELSłAufnahmen charakterisiert (vgl. Abschnitt 3.1.2). Aus der EELS-Analyse kann die chemische Zusammensetzung in der Nähe der Grenzflächen extrahiert werden, sodass zusätzlich zur Rauigkeit aus den HRTEM-Aufnahmen auch die Grenzfläche bezüglich Interdiffusion oder Vermischung der Materialkomponenten untersucht werden kann. Die aus der Gesamtheit der Spektren erhaltenen Konzentrationsprofile können entsprechend der Fick'schen Gesetze der Diffusion ausgewertet werden.

Unter der Annahme zweier halbunendlicher Materialien, die bei $x_{0}$ in Kontakt sind, gilt für das Konzentrationsprofil

$$
c(x, t)=\frac{c_{2}-c_{1}}{2}\left(1+\operatorname{erf}\left(\frac{x-x_{0}}{2 \sqrt{D t_{D}}}\right)\right)
$$

wobei $c_{i}$ die Konzentration des Materials $i, t_{D}$ die Diffusionszeit und $D$ der konzentrationsunabhängige Diffusionskoeffizient ist [Got07].

Die Unschärfe der Grenzfläche $\sigma=\sqrt{2 D t_{D}}$ ergibt sich aus der Standardabweichung der Gaußschen Fehlerfunktion. Die Konzentrationsprofile anhand der EELS-Daten werden entsprechend Gleichung (4.1) angepasst und die Unschärfe $\sigma$ der Grenzfläche bestimmt. In Abbildung 4.6 sind für die drei verwendeten Proben die Konzentrationsprofile und 


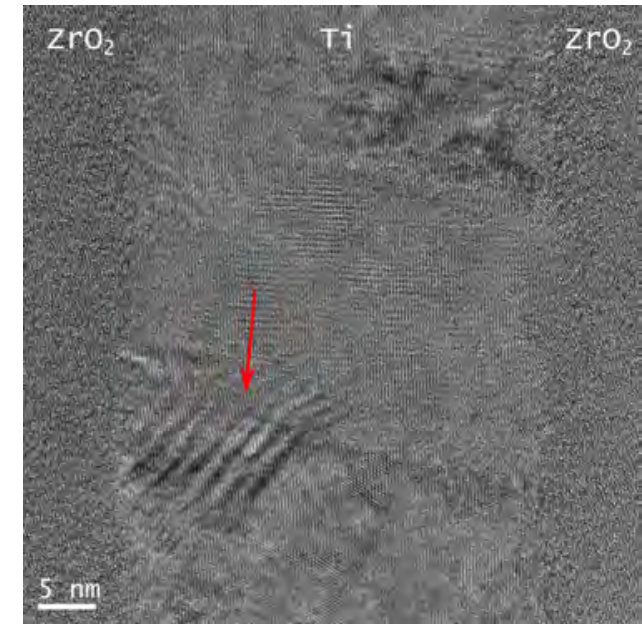

(a) HRTEM Ti50/ZrO 2100

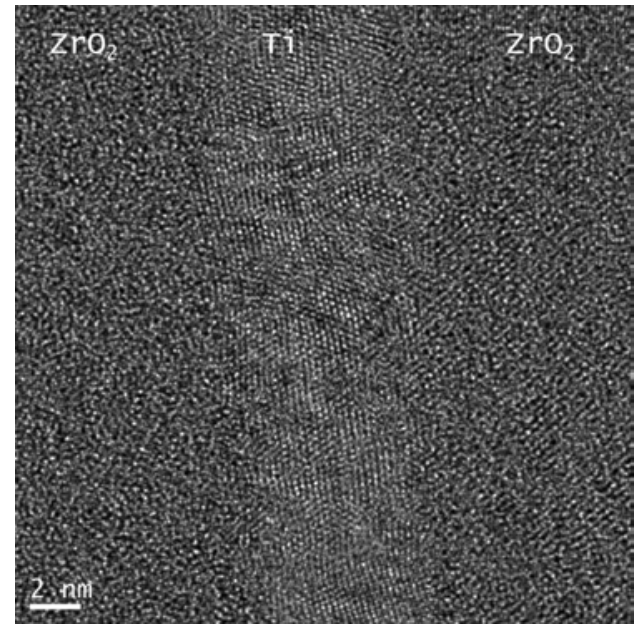

(b) HRTEM Ti10/ZrO 2100

Abbildung 4.5: $\mathrm{HRTEM}$-Aufnahmen Probe $\mathrm{Ti} 50 / \mathrm{ZrO}_{2} 100$ und $\mathrm{Ti10} / \mathrm{ZrO}_{2} 100$, Substrat liegt jeweils links, in $4.5 a$ ist zusätzlich ein Moiré-Muster (markiert mit Pfeil) zu erkennen, welches durch die Beugung des Elektronenstrahls an mindestens zwei übereinander (in der Bildebene) liegenden Körnern, die unterschiedlich zum Elektronenstrahl orientiert sind, erzeugt wird (vgl. [Wil09]).

die entsprechende Anpassung nach Gleichung (4.1) über jeweils eine Grenzfläche dargestellt. Anhand der aufgenommenen Profile über die verschiedenen Grenzflächen einer Probe $\left(\mathrm{ZrO}_{2} /\right.$ Ti- oder $\mathrm{Ti} / \mathrm{ZrO}_{2}$-Grenzfläche) ist keine Asymmetrie bezüglich der Unschärfe der Grenzflächen zu erkennen. In Tabelle 4.2 sind alle aus den Anpassungen der Konzentrationsprofile errechneten Unschärfen der Grenzflächen gezeigt. Im Vergleich der Daten zeigt sich, dass mit wachsender Ti-Schichtdicke die Unschärfe zunimmt. Anhand der erhaltenen Daten ist eindeutig zu erkennen, dass die Unschärfe im Vergleich der Proben Ti50/ZrO 100 und Ti100/ZrO 100 nahezu konstant bleibt, wohingegen eine starke Zunahme der Unschärfe im Vergleich der Proben Ti10/ZrO 100 und $\mathrm{Ti} 50 / \mathrm{ZrO}_{2} 100 \mathrm{zu}$ verzeichnen ist. Dieser Trend zeigt sich unabhängig vom Typ der Grenzfläche als auch des analysierten Materials. Für die Ti10/ZrO 100 -Probe sind die berechneten Unschärfen sehr gering und liegen im Bereich von zwei bis drei TiGitterabständen $\left(a_{c}=0,468 \mathrm{~nm}\right.$ bzw. $a_{a}=0,295 \mathrm{~nm}$ [Lüt00]). Im Vergleich mit dem Gitterparameter $a_{\mathrm{ZrO}_{2}}=0,512 \mathrm{~nm}$ [Ing86] von kristallinem, $3 \mathrm{~mol} \%$-teilstabilisiertem $\mathrm{ZrO}_{2}$ entspricht auch hier die Unschärfe ca. dem eineinhalb- bis zweifachen des Gitterparameters. Es ist jedoch anzumerken, dass die $\mathrm{ZrO}_{2}$-Schichten amorphe Strukturen aufweisen.

Die Analyse der Grenzflächen verschiedener Multilagen-Proben mit variierender Schichtdicke $d_{\mathrm{Ti}}$ im Bereich von $0,38 \mathrm{~nm}$ bis $1,67 \mathrm{~nm}$ und $d_{\mathrm{ZrO}_{2}}$ im Bereich von 2,51 nm bis 9,11 nm mittels Röntgenreflektometrie (engl. X-ray reflectometry, XRR) zeigt ebenfalls eine Abhängigkeit der Unschärfe von der Schichtdicke [Lie07]. Die Analysen der XRR-Messungen liefern wie die EELS-Daten eine ansteigende Unschär- 


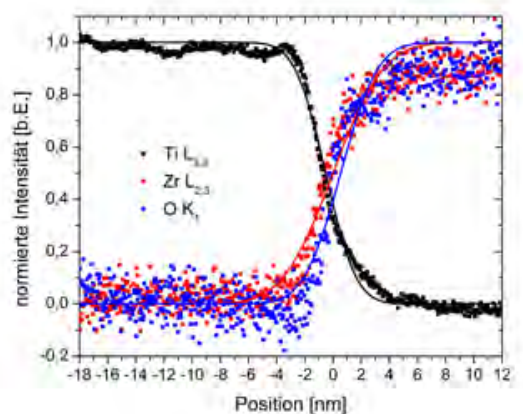

(a) $\mathrm{Ti} / \mathrm{ZrO}{ }_{2}$-Grenzfläche in $\mathrm{Ti} 100 / \mathrm{ZrO}_{2} 100$

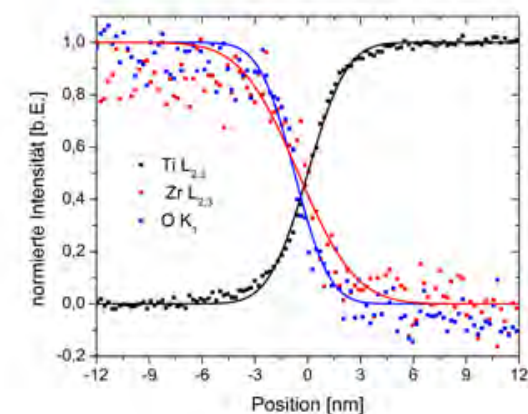

(b) $\mathrm{ZrO}_{2} / \mathrm{Ti}$-Grenzfläche in $\mathrm{Ti50} / \mathrm{ZrO}_{2} 100$

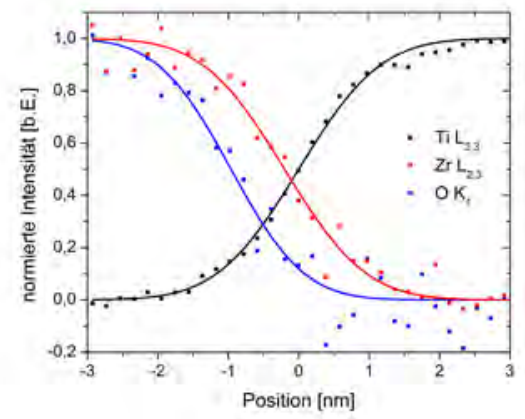

(c) $\mathrm{ZrO}_{2} / \mathrm{Ti}$-Grenzfläche in $\mathrm{Ti10} / \mathrm{ZrO}_{2} 100$

Abbildung 4.6: EELS-Konzentrationsprofile der einzelnen Proben mit den jeweiligen Anpassungen entsprechend Gleichung 4.1

fe beider Grenzflächen mit steigender Ti-Schichtdicke. Die XRR-Messungen ergeben Werte $\sigma_{\mathrm{ZrO}_{2}} / \mathrm{Ti}$ im Bereich von $0,40 \mathrm{~nm}$ bis $0,55 \mathrm{~nm}$ und $\sigma_{\mathrm{Ti} / \mathrm{ZrO}_{2}}$ im Bereich von $0,37 \mathrm{~nm}$ bis $0,51 \mathrm{~nm}$. Es ist jedoch anzumerken, dass die XRR-Daten sowohl die strukturelle Rauigkeit als auch die chemische Vermischung an der Grenzfläche enthalten. Für den Fall einer perfekt scharfen Grenzfläche müssten sich die an die Konzentrationsprofile angepassten Kurven jeweils bei $x=0$ und $y=0$ schneiden. Für die an die Zr-Daten angepassten Kurven ist die Abweichung für alle Proben gering und soll nicht weiter betrachtet werden. Die Sauerstoff-Anpassungen zeigen jedoch deutlichere Abweichungen von der „perfekten“ Grenzfläche, wie zum Beispiel in Abbildung 4.6c zu sehen. Hier ist die angepasste Kurve des Sauerstoffs deutlich zu negativeren x-Werten hin verschoben. Die Verschiebung bedeutet, dass grenzflächennah eine sauerstoffarme Zone in der $\mathrm{ZrO}_{2}$-Schicht vorliegt. Auch für die Konzentrationsprofile der Proben Ti100/ZrO 100 und Ti50/ZrO 100 zeigt sich diese Verschiebung des Sauerstoffprofils. Die hier gefundene schichtdickenabhängige Unschärfe der Grenzflächen wird im Rahmen dieser Arbeit nicht weitergehend analysiert oder diskutiert. Es werden lediglich einige Diskussionsideen für eine weitere Analyse dieses Effekts genannt. Da die Deposition bei Raumtemperatur durchgeführt wurde, ist ein thermisch angeregter diffusiver 


\begin{tabular}{|c|c|c|c|c|c|c|}
\hline \multirow[b]{2}{*}{$\begin{array}{l}\mathrm{Ti} / \mathrm{ZrO}_{2}- \\
\text { Probe }\end{array}$} & \multicolumn{3}{|c|}{$\mathrm{ZrO}_{2} /$ Ti-Grenzfläche } & \multicolumn{3}{|c|}{$\mathrm{Ti} / \mathrm{ZrO}_{2}$-Grenzfläche } \\
\hline & $\begin{array}{c}\sigma_{\mathrm{Ti}} \\
{[\mathrm{nm}]}\end{array}$ & $\begin{array}{c}\sigma_{\mathrm{ZrO}_{2}} \\
{[\mathrm{~nm}]}\end{array}$ & $\begin{array}{c}\sigma_{\mathrm{O}} \\
{[\mathrm{nm}]}\end{array}$ & $\begin{array}{c}\sigma_{\mathrm{ZrO}_{2}} \\
{[\mathrm{~nm}]}\end{array}$ & $\begin{array}{c}\sigma_{\mathrm{Ti}} \\
{[\mathrm{nm}]}\end{array}$ & $\begin{array}{c}\sigma_{\mathrm{O}} \\
{[\mathrm{nm}]}\end{array}$ \\
\hline $100 / 100$ & $2,39(16)$ & $3,39(43)$ & $4,71(45)$ & $3,45(36)$ & $2,44(24)$ & $3,07(42)$ \\
\hline $50 / 100$ & $1,99(14)$ & $2,01(32)$ & $3,40(83)$ & $2,99(11)$ & $2,36(14)$ & $2,67(44)$ \\
\hline $10 / 100$ & $0,93(2)$ & $1,21(8)$ & $0,73(5)$ & $1,28(11)$ & $0,78(1)$ & $0,67(6)$ \\
\hline
\end{tabular}

Tabelle 4.2: Aus EELS-Konzentrationsprofilen bestimmte Unschärfen der Grenzflächen, die angegebenen Werte verstehen sich jeweils als Mittelwert der verschiedenen Profile mit dem jeweiligen Standardfehler des Mittelwertes

Prozess als Ursache der Unschärfe der Grenzfläche unwahrscheinlich XX195.

Als wahrscheinlicher ist eine ballistische Diffusion anzusehen, bei der durch den Beschuss mit hochenergetischen Ionen eine Mischung von Atomen an einer Grenzfläche entstehen kann. Für verschiedene Metall/Keramik-Grenzflächen ist eine Mischung der Atome an der Grenzfläche unabhängig von der chemischen Bindung gefunden worden $\left(\mathrm{Ar}^{+}\right.$-Ionen mit $\left.150 \mathrm{keV}\right)$ Nag99. In der PLD werden ähnliche Partikelenergien erreicht, sodass eine ballistische Mischung an den Grenzflächen nicht ausgeschlossen werden kann.

Weiterhin kann eine Asymmetrie zwischen Sauerstoff und Zirkon an den Grenzflächen beobachtet werden. Diese Asymmetrie lässt auf eine sauerstoffdefizitäre Region in der Nähe der Grenzfläche innerhalb der Zirkon-Schicht schließen. Die Breite dieser defizitären Schicht liegt im Bereich von $1 \mathrm{~nm}$ bis $2 \mathrm{~nm}$. Abgesehen von diesem Defizit an der Grenzfläche sind die $\mathrm{ZrO}_{2}$-Schichten aufgrund des PLD-Herstellungsprozesses leicht sauerstoffdefizitär $(Z r / O \approx 1 / 1,8)$ Hei10, Lie12]. Die grenzflächennahe, sauerstoffdefizitäre Region in den $\mathrm{ZrO}_{2}$-Schichten erklärt sich wahrscheinlich durch eine Interreaktion des Sauerstoffes und des Titans, so kann bereits bei geringen Temperaturen der Sauerstoff mit dem Titan reagieren bzw. sich vermischen. Eine solche Reaktion würde zu einem Sauerstoff-Defizit in den $\mathrm{ZrO}_{2}$-Schichten führen. Die Standardbildungsenthalpie von Titanoxid ist geringer als die von Zirkonoxid [Cha98], sodass eine Reaktion zwischen Titan und Sauerstoff nicht ausgeschlossen werden kann.

\subsection{FEM-Simulationen der Bruchgeometrie}

Die Simulation der Spannungszustände der idealen Geometrie der Bruchversuche, sowie auch der abweichenden Geometrien, dient zur Analyse der Einflüsse einer nicht perfekten Geometrie oder Durchführung der Experimente. Durch eine asymmetrische Geometrie oder auch Belastung der einzelnen Si-Säulen kann es eine Vorzugsrichtung bei der Rissbildung oder der späteren Rissausbreitung geben. Durch den Vergleich der 


\begin{tabular}{ll} 
Simulation & Parameter \\
\hline \hline sym-100 & symmetrische Verformung 100 nm und Si-Säulen \\
sym-100-t & sym. Verformung und Si-Säulen, vertauschte Materialien \\
asym-100 & asymmetrische Verformung $(1: 3,1: 5,1: 1,5)$, sym. Si-Säulen \\
xy-100 & Verformung in x- und y-Richtung, sym. Si-Säulen \\
asym-100-p & asym. Si-Säulen, sym. Verformung 100 nm
\end{tabular}

Tabelle 4.3: Übersicht der durchgeführten Simulationen mit COMSOL

verschiedenen Simulationen zeigt sich, ob und wie ausgeprägt eine Vorzugsrichtung durch eine solche Asymmetrie hervorgerufen werden kann.

In Tabelle 4.3 ist eine Übersicht der verschiedenen Simulationen sowie deren im weiteren Verlauf genutzte Nomenklatur gegeben.

Um die Vergleichbarkeit der unterschiedlichen Simulationen untereinander zu gewährleisten, werden die Spannungszustände bei ähnlichen Öffnungsweiten der Kerbe analysiert. Die Öffnungsweite der Kerbe ist in allen Simulationen ca. $100 \mathrm{~nm}$. Die notwendigen Verformungen der Si-Säulen, um die Öffnungsweite der Kerbe zu erreichen, sind abhängig von den in den jeweiligen Simulationen gewählten Randbedingungen. In Abbildung 4.7 sind die notwendigen Verformungen der Säulen zu sehen, um eine Öffnung der Kerbe um $100 \mathrm{~nm}$ zu erreichen.

In den nachfolgenden Abbildungen werden die Von-Mises-Oberflächenspannungen farblich kodiert gezeigt. Die Simulationen werden entsprechend der in Tabelle 4.3 eingeführten Nomenklatur benannt.

Die Spitze der Kerbe liegt in allen nachfolgend dargestellten Simulationen in einer Ti-Schicht. Die Simulation mit vertauschten Materialien zeigt identische Spannungsverteilungen, sodass hier nur der Fall der Kerbe im Ti gezeigt wird.

In Abbildung 4.8 ist der Spannungszustand der symmetrischen Simulation gezeigt. Diese Geometrie sowie die Belastung der Säulen ist hier perfekt symmetrisch, sodass diese Simulation den Idealzustand der in-situ Bruchversuche darstellt. In Abbildung 4.8a ist eine dreidimensionale Übersicht der gesamten Lamelle gezeigt. Die Spannungsverteilung zeigt ein erwartetes Verhalten. An der Spitze der Kerbe ist die Spannung am höchsten und zeigt die typische Form der Spannungsverteilung eines Mode I Risses ähnlich der Form der Zahl 8 (vgl. z.B. And95]). Eine vergrößerte Abbildung im Bereich der Spitze der Kerbe ist in Abbildung 4.8b gezeigt. Hier zeigt sich die Symmetrie der Spannungsverteilung sehr deutlich. Zusätzlich zu der Beobachtung des erwarteten Spannungszustandes im Bereich der Kerbe ist weiterhin auffällig, dass es zu Spannungskonzentrationen an den Verbindungsstellen zwischen dem dünnen Bereich der Lamelle und den Si-Säulen kommt. Die Spannungen sind hier nicht so hoch wie an der Kerbe, jedoch überschreiten sie die Fließspannung des Titans $\left(\sigma_{y s, 100}=2,4 \mathrm{GPa}\right)$. Es ist daher 


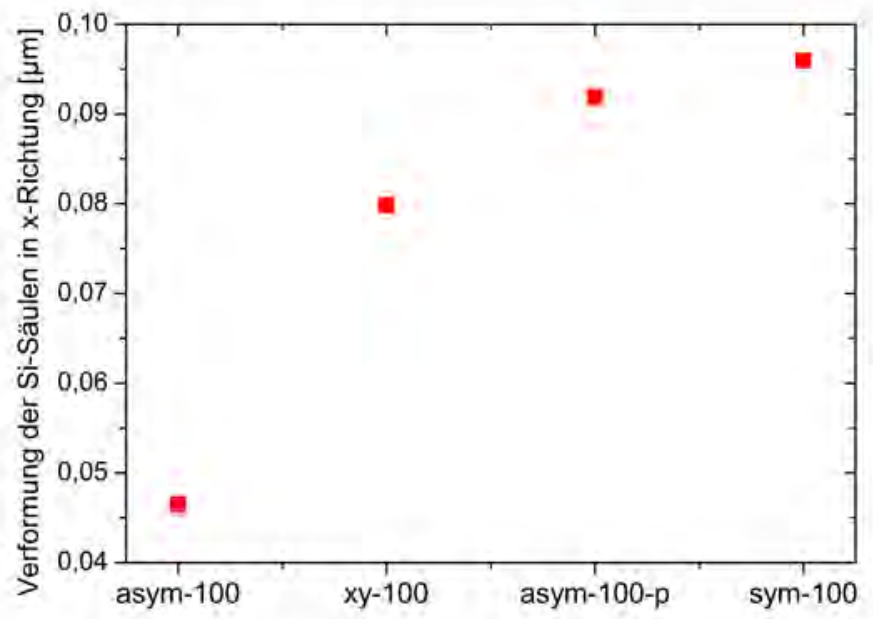

Abbildung 4.7: Die notwendigen Verformungen der Si-Säulen in den verschiedenen Simulationen um eine vergleichbare Öffnungsweite der Kerbe zu erhalten. Für Simulation asym-100 ist nur ein Verformungsweg aufgetragen. Bei der gewählten Asymmetrie der Verformung vom 1:3 beträgt die Verformung der anderen Säule $0,9 \mu \mathrm{m}$.

nicht ausgeschlossen, dass es während der Bruchversuche an den Verbindungsstellen zu plastischer Verformung und im ungünstigsten Fall auch zur Rissbildung kommen könnte.

In Abbildung 4.9 sind die drei Simulationen mit asymmetrischer Geometrie und Belastung dargestellt. Die Spannungsverteilung an der Kerbe (Abbildung 4.9b, größere Säule liegt unten) für die Simulation mit asymmetrischer Säulengröße zeigt keine signifikante Abweichung von dem symmetrischen Fall in Abbildung 4.8b. Der Spannungszustand unterscheidet sich lediglich an den Verbindungspunkten der Multilage zu der größeren Säule. Hier ist die Spannung auf der Höhe der Kerbe etwas höher als am gegenüberliegenden Verbindungspunkt zu der anderen Säule. Außerdem ist die Spannungskonzentration am oberen Ende (positive z-Richtung in Abb. 4.9b) an der größeren Säule weniger ausgeprägt im Vergleich zu der anderen Säule. Diese Abweichung vom Spannungszustand des symmetrischen Falls ist nicht signifikant, sodass leicht unterschiedliche Säulendimensionen die experimentellen Ergebnisse nicht merklich beeinflussen sollten.

Abbildung 4.9c zeigt den Spannungszustand an der Kerbe bei einer asymmetrischen Belastung der Säulen im Verhältnis 1:3. Die obere Säule ist dementsprechend dreimal mehr verformt im Vergleich zur unteren. Die Asymmetrie der Verformung, die in den Experimenten durch eine schlecht ausgerichtete STM-Spitze relativ zu den Si-Säulen erzeugt werden könnte, hat eine deutliche Veränderung des Spannungszustandes im Vergleich zu dem symmetrischen Fall zur Folge. Es ist eindeutig eine Rotation des gesamten Spannungszustandes in der xz-Ebene zu erkennen. Da die Farbskalen alle gleich 


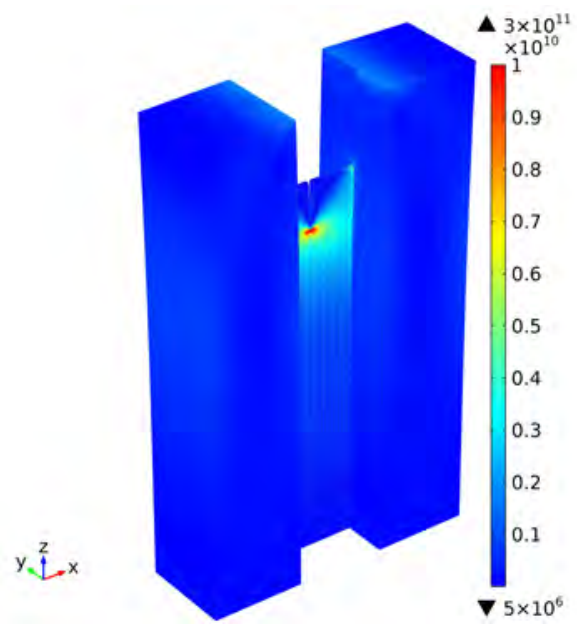

(a)

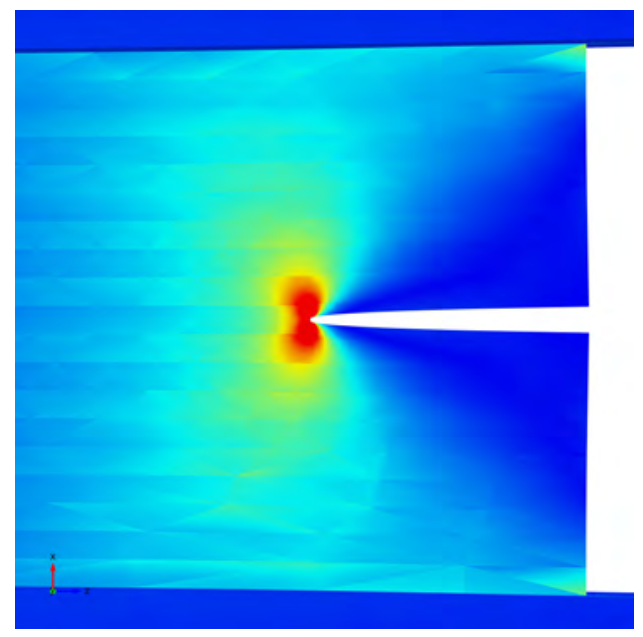

(b)

Abbildung 4.8: Spannungsverteilung der symmetrischen Simulation mit $100 \mathrm{~nm}$ Auslenkung der SiSäulen (sym-100). Farbskala ist identisch für beide Abbildungen, Spannungswerte in $\mathrm{Pa}$

gewählt sind, ist zu erkennen, dass sich das Spannungsfeld verkleinert hat im Vergleich zu Abbildung 4.8b. Auch die Spannungskonzentrationen an den Verbindungspunkten zur Säule sind weniger stark ausgeprägt. Im oberen Teil der Lamelle hingegen erstreckt sich das Spannungsfeld über eine größere Distanz und ist in positive z-Richtung gedreht. Der Anteil der Lamelle, in dem die Spannungen die Fließspannung des Titans erreichen, ist deutlich größer, sodass hier mit mehr Plastizität zu rechnen ist. Durch die Rotation des Spannungszustandes hin zur stärker verformten Seite erhöht sich die Wahrscheinlichkeit einer bevorzugten Rissbildung und -bewegung in Richtung stärkere Verformung. Zusätzlich erzeugt die Asymmetrie der Verformung eine wesentlich stärkere Spannungskonzentration am Verbindungspunkt zwischen Lamelle und der mehr verformten Säule, welche eine zweite mögliche Position zur Rissbildung darstellt. Simulationen mit variierendem Verhältnis der Belastung (1:1,5 und 1:5) ergeben keine neuen Ergebnisse.

Eine weitere Asymmetrie könnte durch eine verdrehte STM-Spitze relativ zu den Säulen entstehen. Diese Situation ist in der Simulation in Abbildung 4.9d dargestellt. Die obere Säule ist hier gleich stark in positive $\mathrm{x}$ - und $\mathrm{y}$-Richtung (aus der Bildebene heraus) verformt. Die untere Säule ist entsprechend in -x- und -y-Richtung verformt. Durch die Asymmetrie in der y-Richtung wird hier zusätzlich zur eigentlichen Zugspannung auf die Kerbe auch eine Torsion ausgeübt, sodass es kein reiner Mode I Bruchversuch mehr ist. Der Spannungszustand bleibt jedoch weitestgehend ähnlich zu dem symmetrischen Fall in Abbildung 4.8b. Die Spannungsverteilung ist hin zur oberen Säule verschoben und etwas verdreht. Die Rotation ist aber wesentlich geringer als in Abbildung 4.9c. Die Verschiebung zur oberen Säule ist hingegen vergleichbar zu Abbildung 4.8b. Auch die zweidimensionale Verformung erzeugt Spannungen im Bereich der Fließspannung 


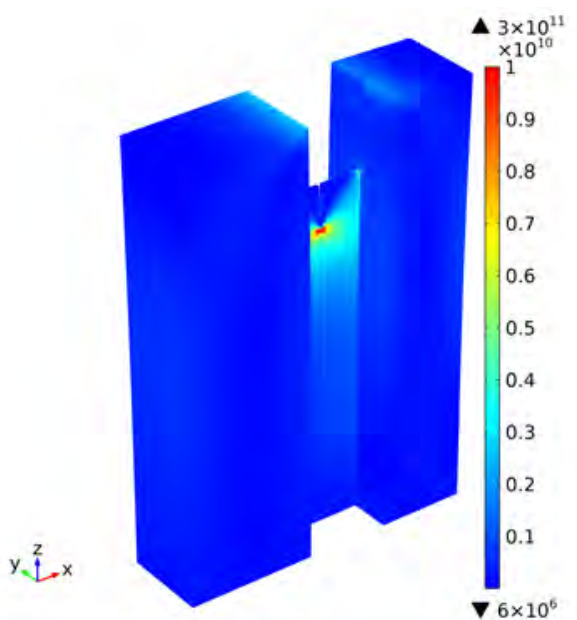

(a) asym-100-p

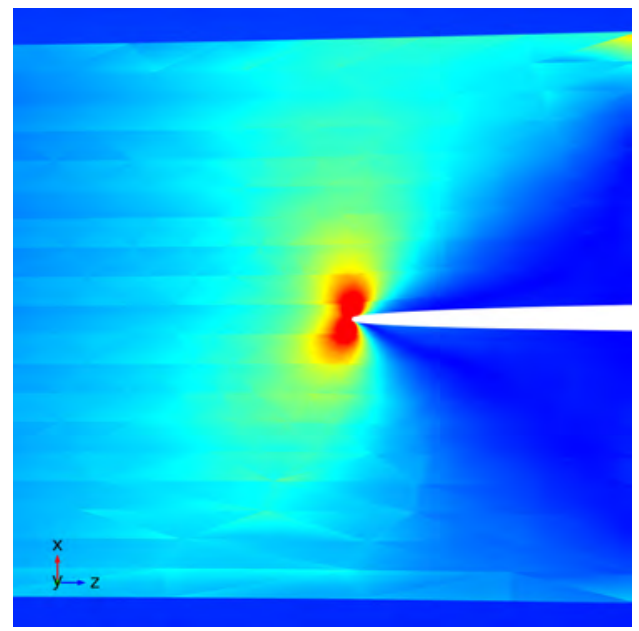

(c) asym-100

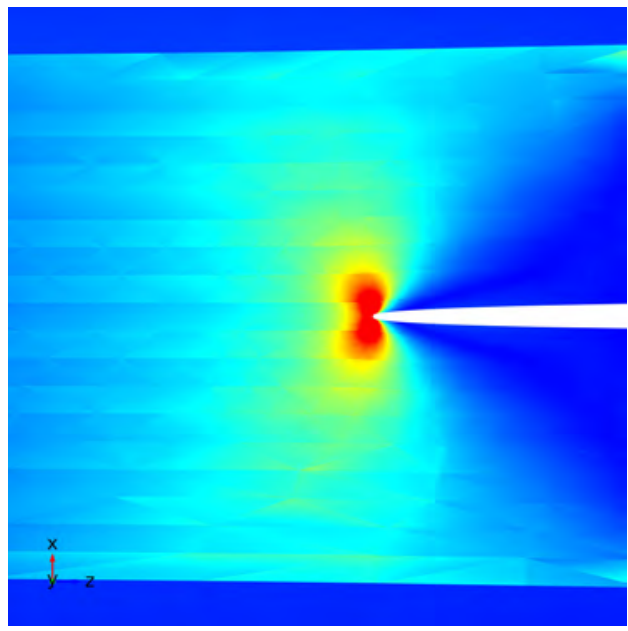

(b) asym-100-p

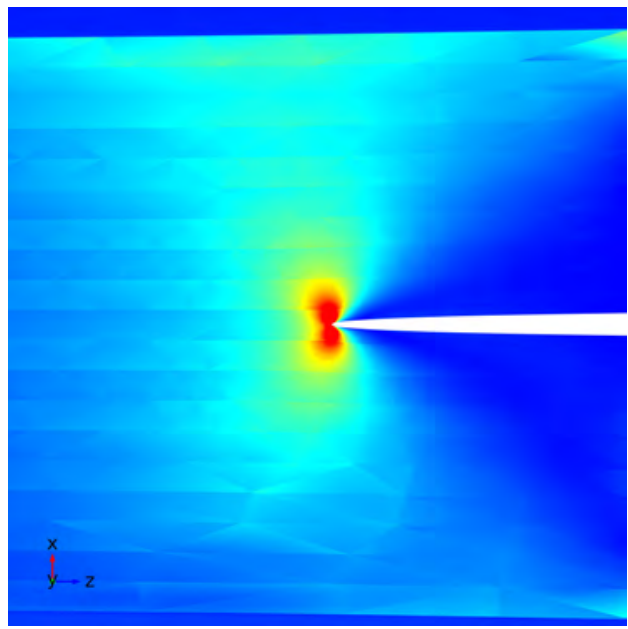

(d) $x y-100$

Abbildung 4.9: Spannungsverteilung der asymmetrischen Simulationen asym-100, -p und xy-100 (Benennung entsprechend Tabelle 4.3); Die Farbskala der Spannungen aus 4.9a gilt auch für die anderen hier gezeigten Spannungsverteilungen.

des Titans über den gesamten oberen Bereich der Lamelle. Die Spannungskonzentration am Verbindungspunkt zur Säule ist stärker ausgeprägt als im symmetrischen Fall. Da der Spannungszustand an der Spitze der Kerbe jedoch vergleichbar zu Abbildung $4.8 \mathrm{~b}$ ist, ist hier nicht mit einer Vorzugsrichtung bei der Rissbildung und -bewegung zu rechnen.

Zusammenfassend zeigen die durchgeführten FEM-Simulationen, dass es nur bei einer asymmetrischen Belastung der Si-Säulen zu einer Vorzugsrichtung bei der Rissbildung oder einem Riss am Rand der Lamelle an den Verbindungspunkten zu den Si-Säulen kommen könnte. Alle anderen simulierten Asymmetrien bewirken keine signifikanten Veränderungen der Spannungszustände an der Spitze der Kerbe. Die Effekte durch die Asymmetrie sind im Verhältnis zu möglichen Auswirkungen der Anisotropie der elastischen Konstanten und damit der Verformung und dem Rissverhalten als gering 
einzustufen. Die Farbskalen sind in allen Abbildungen 4.8 und 4.9) identisch gewählt, sodass daraus ersichtlich ist, dass sich die Höhe der erreichten Spannungen an der Spitze der Kerbe oder in der Lamelle nicht ändert. Dem hingegen liegt der Elastizitätsmodul des Titans aufgrund der Anisotropie der hexagonalen Kristallstruktur zwischen $100 \mathrm{GPa}$ bis $145 \mathrm{GPa}$ [üt00], sodass dieser Effekt wahrscheinlich als größer und entscheidender für das Rissverhalten anzusehen ist. 


\subsection{Bruchversuche an Einzelschichten}

Nachfolgend werden die in-situ Bruchversuche der jeweiligen Einzelkomponenten des Multilagensystems dargestellt. Diese Versuche dienen als Kontroll- und Vergleichsexperimente, um das Bruchverhalten der Materialien in den Multilagen besser diskutieren zu können. Im Fall der $\mathrm{ZrO}_{2}$-Einzelschicht wird zusätzlich ein Bruchversuch an einer kristallisierten Schicht dargestellt.

Die Bruchversuche werden anhand verschiedener Verformungsschritte beschrieben. Die Unterteilung ergibt sich allein aus der Tatsache, dass es nicht möglich ist, mehr als 500 Einzelbilder in einer Bildserie zusammenzufassen. Diese Limitierung gilt für alle in der Dissertation durchgeführten Bruchversuche.

\subsubsection{Titan-Schicht}

Die Ti-Einzelschicht ist ebenfalls mittels der PLD hergestellt worden. Die Schichtdicke beträgt ca. 2,5 $\mu \mathrm{m}$. Ein kollumnares Wachstum wie in den Multilagen kann nicht beobachtet werden. Vielmehr zeigt diese Ti-Einzelschicht polykristallines Wachstum. Die mittels Röntgenbeugung (engl. X-ray diffraction, XRD) bestimmte Korngröße dieser Schicht beträgt ca. $35 \mathrm{~nm}$. Die Bruchgeometrien werden, wie in Abschnitt 3.3.1 dargestellt, präpariert und in-situ im TEM getestet und analysiert. Nachfolgend werden für die Ti-Einzelschicht die Ergebnisse beider Orientierungen gezeigt.

\section{Parallel zur Wachstumsrichtung (in plane)}

In Abbildung 4.10 sind TEM-Aufnahmen der Ti-Schicht in der Geometrie der Rissausbreitung parallel zur Wachstumsrichtung dargestellt. Dieser Bruchversuch ist in sechs einzelne Verformungsschritte unterteilt. Abbildung 4.10a zeigt eine Hellfeldaufnahme (engl. bright field micrograph, BF) der Kerbe vor dem Bruchversuch. In Abbildungen $4.10 \mathrm{~b}$ und 4.10c sind zwei weitere Hellfeldaufnahmen während des Bruchversuchs (nach dem 2. und 5. Verformungsschritt) gezeigt.

$\mathrm{Zu}$ Beginn des Bruchversuchs ist während des ersten Verformungsschrittes lediglich eine Vergrößerung der Öffnungsweite der anfänglichen Kerbe zu erkennen. Die Bildung eines Risses an der Spitze der Kerbe kann nicht beobachtet werden. Während der ersten Belastung der Probe bewegen sich kontinuierlich Biegekonturen in der Lamelle, die eine Reaktion auf die Verformung darstellen. Sie dienen als eindeutiges Indiz, dass eine Verformung der Lamelle stattfindet. Hier muss jedoch festgehalten werden, dass diese Bewegung der Biegekonturen auch von einer Verbiegung der Lamelle in der Bildebene herrühren könnte.

Am Ende des zweiten Verformungsschrittes ist ein sehr kurzer Riss an der Spitze der 


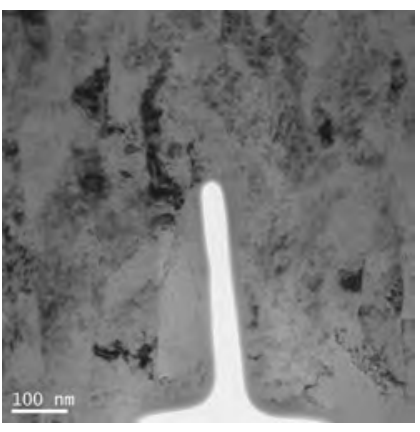

(a) BF vorher

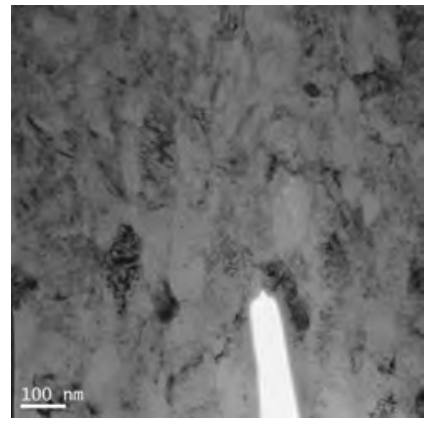

(b) Nach 2. Belastung

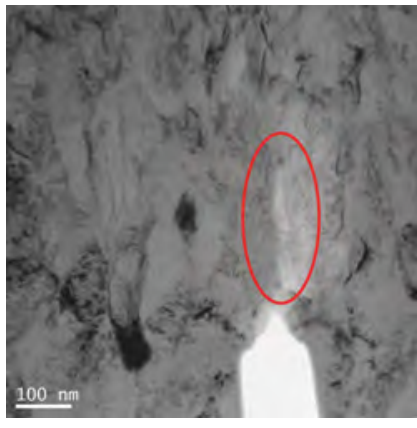

(c) Nach 5. Belastung

Abbildung 4.10: Bruchversuch einer Ti-Einzelschicht parallel zur Wachstumsrichtung, STM-Spitze wird von unten angenähert

Kerbe zu beobachten. Die Bildung dieses Risses geht mit der Beobachtung von schnellen Kontrastwechseln bzw. Versetzungsaktivität in der Nähe der Spitze der Kerbe einher. Während des dritten und des vierten Verformungsschrittes kann weiterhin Versetzungsaktivität im Bereich um die Spitze der Kerbe bzw. des kleinen gebildeten Risses beobachtet werden. Der Riss wächst in diesen beiden Schritten nur wenige Nanometer. Die Öffnungsweite steigt während der sukzessiven Verformung stetig an.

Im fünften Verformungsschritt wird eine deutlich verstärkte Versetzungsaktivität im Bereich direkt vor der Rissspitze beobachtet (Markierung in Abbildung 4.10c). Der Bereich verstärkter Aktivität erstreckt sich über ca. $250 \mathrm{~nm}$ bis $300 \mathrm{~nm}$ in Richtung der Rissausbreitung. Zusätzlich zu den schnellen Kontrastwechseln kann in diesem Bereich eine kontinuierlich ansteigende Helligkeit in den einzelnen TEM-Aufnahmen der entsprechenden Bildserie verzeichnet werden. Diese lässt darauf schließen, dass die Lamelle in dem Bereich dünner in Richtung des Elektronenstrahls wird. Die lokale Verringerung der Dicke der Lamelle setzt sich in dem letzten Verformungsschritt fort, bis es zwischen zwei einzelnen Bildern zum katastrophalen Versagen der Ti-Schicht kommt. Auch während der letzten Verformung ist weiterhin Versetzungsaktivität über den gesamten abgebildeten Bereich der Lamelle zu beobachten.

In Abbildung 4.11 ist eine Übersichtsaufnahme des gesamten Risses gezeigt. Anhand der Abbildung ist zu erkennen, dass sich der Riss relativ gerade durch die gesamte Ti-Schicht bewegt hat. In dem Bereich, in dem die Lamelle während des Bruchversuchs lokal dünner geworden ist, ist die Oberfläche des Risses etwas rauer im Vergleich zum Rest der Rissoberfläche. Die raue Oberfläche in diesem Bereich lässt in Verbindung mit der beobachteten Helligkeitsveränderung auf ein duktiles Bruchverhalten schließen, was für Metalle zu erwarten ist. Die weitere Oberfläche des Risses lässt eher auf einen Spaltbruch schließen, wie er üblicherweise in spröden Materialien gefunden wird. 


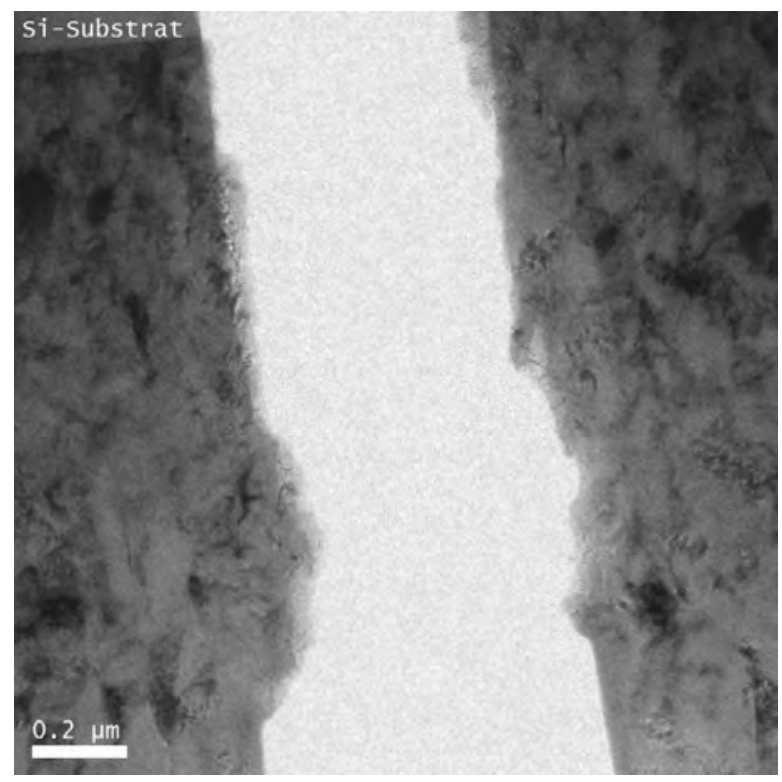

Abbildung 4.11: Bruchversuch Ti-Einzelschicht; BF-Übersichtsaufnahme des Risses nach dem katastrophalen Versagen der Ti-Schicht, STM-Spitze wird von unten angenähert

\section{Senkrecht zur Wachstumsrichtung (out of plane)}

Im Folgenden werden die Bruchversuche der Ti-Einzelschicht senkrecht zur Wachstumsrichtung gezeigt. Diese Versuche werden entsprechend der bereits geschilderten Methodik für Bruchversuche entlang der Grenzflächen (Abschnitt 3.3.1) durchgeführt. In Abbildung 4.12 sind zwei Hellfeldaufnahmen während des Bruchversuchs zu sehen. Der Bruchversuch ist in vier Verformungsschritte unterteilt, wobei im ersten Schritt lediglich der Kontakt zwischen den Si-Säulen und der STM-Spitze aufgebaut wird und sich die ersten Biegekonturen bewegen. In diesem Schritt findet noch kein Risswachstum statt.

Im zweiten Schritt hingegen ist eine Vergrößerung der Öffnungsweite der Kerbe sowie eine Abstumpfung der Spitze der Kerbe zu beobachten. Die Veränderungen der Kerbe gehen, wie schon in dem vorherigen Ti-Bruchversuch, mit der Aktivität von Versetzungen einher. Die Versetzungsaktivität ist im Bereich der Spitze der Kerbe (Markierung in Abbildung 4.12a) höher im Vergleich zu den Randbereichen.

Abbildung 4.12b zeigt die Konfiguration am Ende des dritten Verformungsschrittes. Im Vergleich zum Ende des zweiten Verformungsschrittes (Abb. 4.12a) ist ein deutliches Risswachstum ausgehend von der Spitze der Kerbe zu erkennen. Der Riss ist in diesem Schritt über eine Länge von ca. $100 \mathrm{~nm}$ gewachsen. Die genaue Position der Rissspitze ist jedoch nur schwer auszumachen. Zusätzlich ist wiederum ein lokal dünnerer Bereich vor der eigentlichen Rissspitze zu beobachten (Markierung Abb. 4.12b). In diesem Bereich ist das Material noch nicht gerissen, sondern noch intakt. Das Titan ist hier stark gedehnt und dadurch dünner als in den Randbereichen, sodass ein hellerer Kontrast zu 


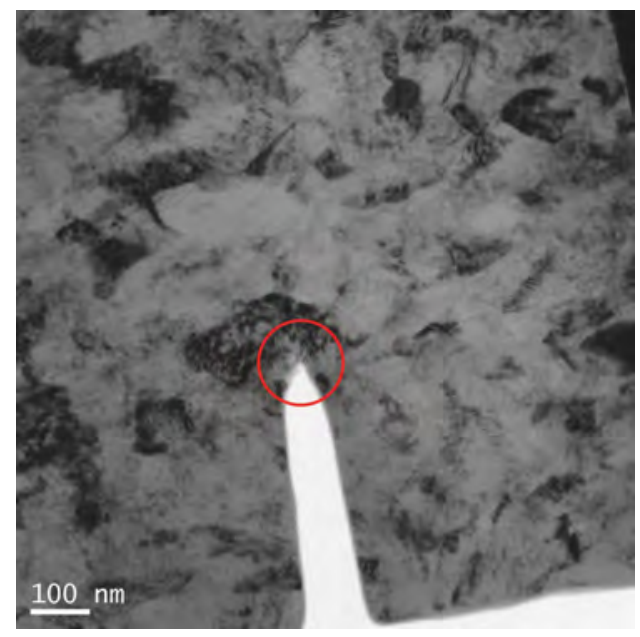

(a) Nach 2. Verformung

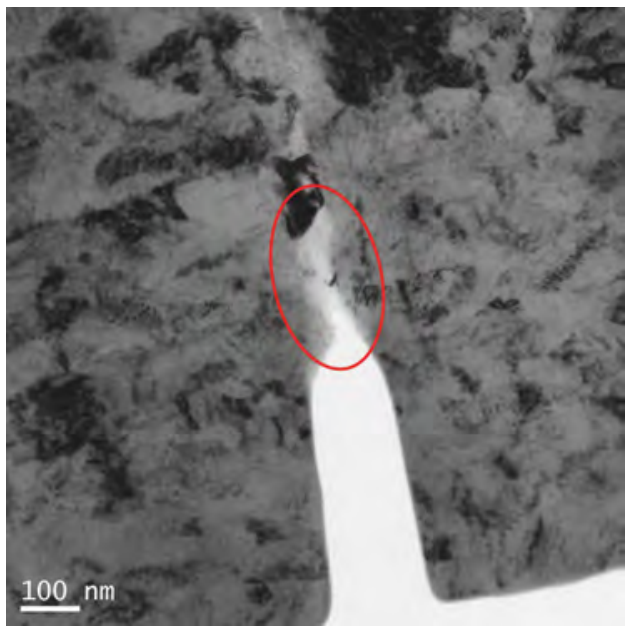

(b) Nach 3. Verformung

Abbildung 4.12: Bruchversuch einer Ti-Einzelschicht senkrecht zur Wachstumsrichtung

beobachten ist.

Während des letzten Verformungsschrittes (hier nicht gezeigt) wächst der Riss kontinuierlich weiter mit steigender Verformung und läuft daher aus dem Bildausschnitt heraus. Wie in allen vorherigen Schritten ist auch in diesem letzten Schritt eine massive Versetzungsaktivität zu beobachten, die in der Nähe des Risses deutlich stärker ist. Eine Übersicht über den gesamten Riss ist in Abbildung 4.13a gezeigt. Im Vergleich zum Rissverhalten parallel zur Wachstumsrichtung (siehe Abbildung 4.11) ist hier auffällig, dass es zu keinem katastrophalen Versagen der Ti-Schicht kommt und dass sich der Riss nicht so gerade ausgebreitet hat. Vielmehr wird der Riss mehrmals von einer geraden Ausbreitungsrichtung abgelenkt. Zusätzlich wird beobachtet, dass es zur Rissüberbrückung kommt.

In Abbildungen 4.13b und 4.13c sind eine Hell- und eine Dunkelfeldaufnahme in höheren Vergrößerungen gezeigt (quadratische Markierung in Abbildung 4.13a). Es ist eindeutig zu erkennen, dass es neben einigen Hohlräumen in der Ti-Schicht noch intakte Ti-Bereiche gibt, obwohl sich der Riss bereits weiter ausgebreitet hat. Die Rissüberbrückung ist an mindestens vier Positionen über die Lamelle verteilt zu beobachten. Anhand der Dunkelfeldaufnahme zeigt sich, dass die brückenbildenden Ti-Stücke erleuchtet und damit identisch zum Elektronenstrahl orientiert sind, sodass sie zu einem einzigen Korn gehören könnten. An den anderen Rissüberbrückungspositionen zeigt sich eine vergleichbare Konfiguration.

Der Riss bzw. der verformte Bereich erstreckt sich über eine Länge von insgesamt ca. $3,2 \mu \mathrm{m}$. Jedoch ist im eingekreisten Bereich (siehe Abbildung 4.13a) der letzte eindeutige Hohlraum mit zwei separierten Oberflächen zu erkennen. Mit dieser Stelle als Rissspitze ist die erreichte Risslänge ca. 1,9 $\mathrm{m}$. 


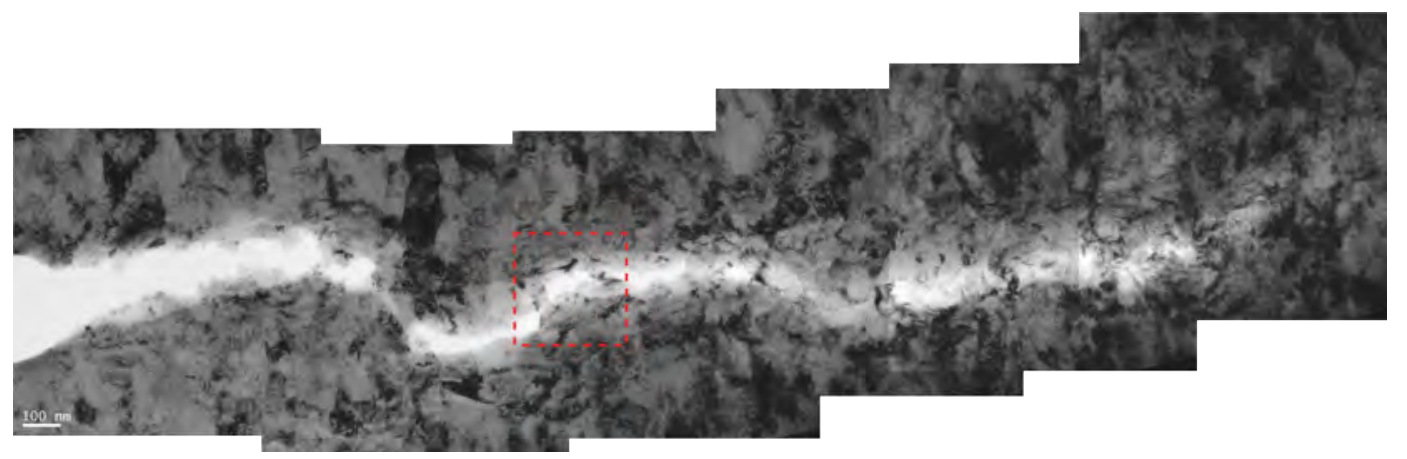

(a) Übersicht Riss

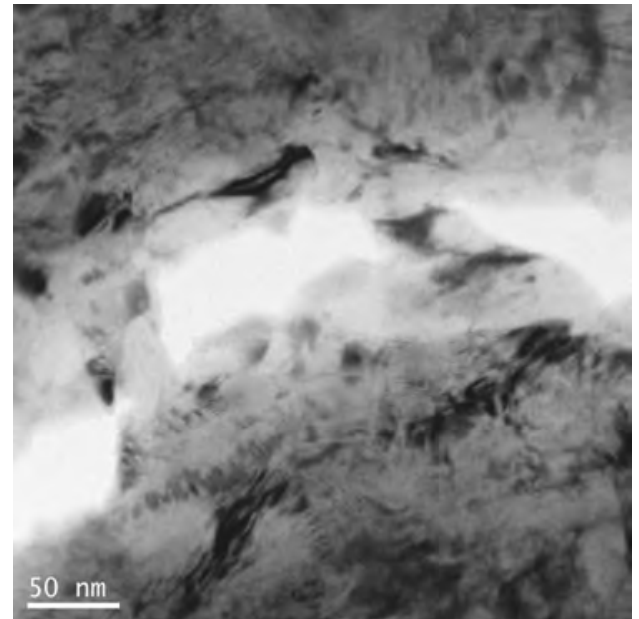

(b) BF Markierung

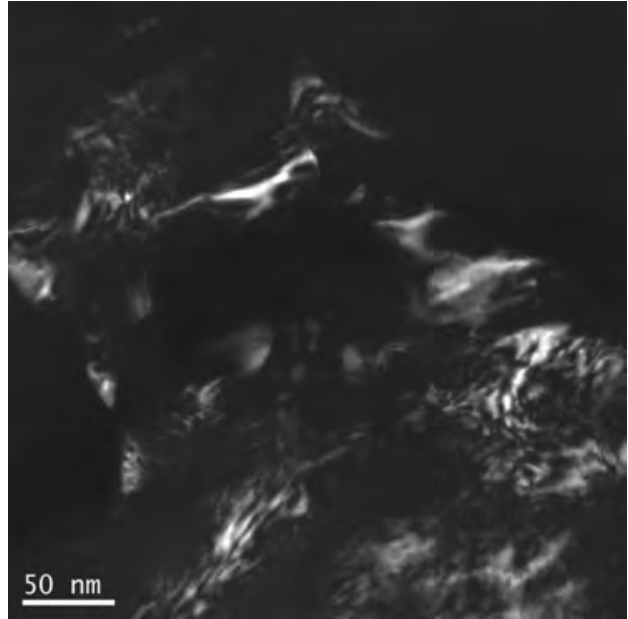

(c) DF Markierung

Abbildung 4.13: Bruchversuch einer Ti-Einzelschicht senkrecht zur Wachstumsrichtung

\begin{tabular}{lccc} 
Orientierung & $r_{p}[\mathrm{~nm}]$ & $J_{I Q}\left[\mathrm{~J} \mathrm{~m}^{-2}\right]$ & $K_{I Q}\left[\mathrm{MPa} \mathrm{m}^{1 / 2}\right]$ \\
\hline \hline parallel (in-plane) & $593(30)$ & $3,815(354)$ & $0,759(181)$ \\
senkrecht (out-of-plane) & $613(6)$ & $6,640(578)$ & $1,001(224)$
\end{tabular}

Tabelle 4.4: Plastische Zone, Energiefreisetzungsrate und Bruchzähigkeit Ti-Einzelschichten

\section{Plastische Zone und Energiefreisetzungsrate}

Die Größen der plastischen Zonen $r_{p}$ der beiden Ti-Einzelschichten in der Richtung der Rissausbreitung werden wie in Abschnitt 3.4 .2 anhand der verschiedenen Bildserien der einzelnen Verformungsschritte geschätzt. Die in Tabelle 4.4 angegebenen Werte verstehen sich als Mittelwerte über die plastischen Zonen verschiedener Bildserien.

Weiterhin sind die kritischen Werte der Energiefreisetzungsrate sowie der Bruchzähigkeit (nach Gleichung (3.3) ) bei der Rissbildung dargestellt. Exemplarisch sind in Abbildung 4.14 die Risswiderstandskurven für beide Ti-Einzelschichten gezeigt. 


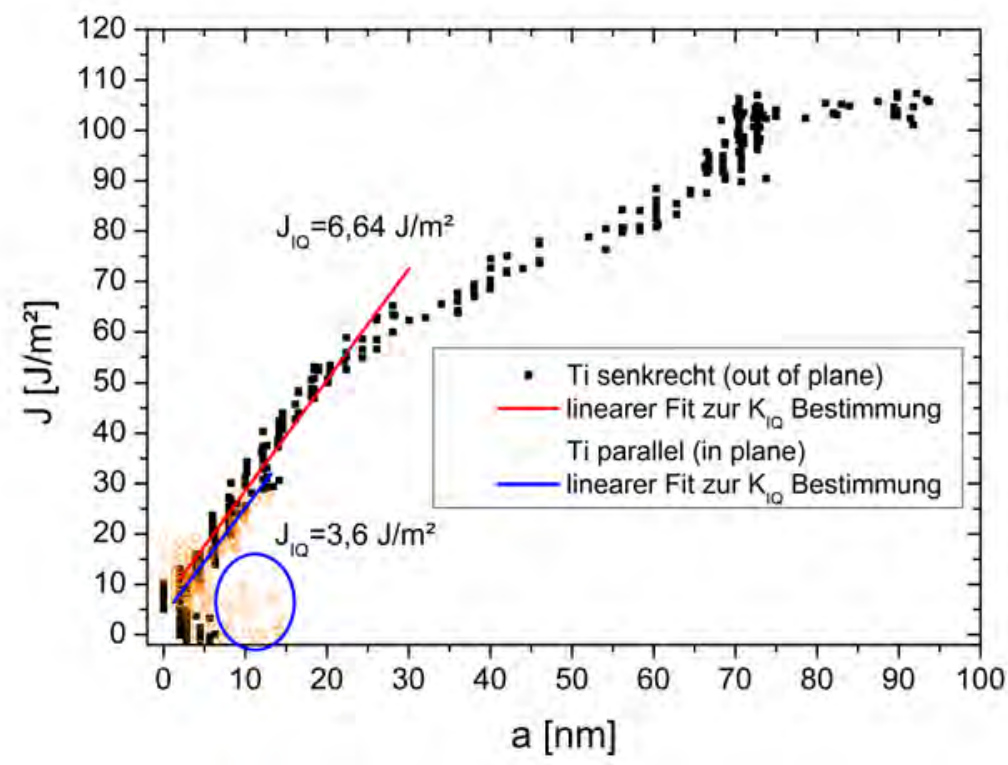

Abbildung 4.14: Energiefreisetzungsrate vs. Risslänge der Ti-Einzelschichten, blau markierte Punkte sind Artefakte der automatischen Auswertung und werden für die lineare Anpassung zur Bestimmung der kritischen Bruchzähigkeit vernachlässigt

\subsubsection{Zirkonoxid-Schicht}

Wie schon im Fall des Titans, ist auch eine Zirkonoxid Einzelschicht hergestellt worden, um die mechanischen Eigenschaften des amorphen $\mathrm{ZrO}_{2}$ untersuchen zu können. Diese Schicht ist ebenfalls ca. $3 \mu \mathrm{m}$ dick. Sie wird entsprechend mit einer SiDeckschicht versehen und auf ihr Bruchverhalten untersucht. Zusätzlich wird eine amorphe $\mathrm{ZrO}_{2}$-Einzelschicht ausgelagert, um die Kristallisation zu ermöglichen. Diese kristalline Schicht wird anschließend im Vergleich zur amorphen Schicht auch im Bruchtest untersucht.

\section{Amorphe Schicht}

In Abbildung 4.15a ist eine Hellfeldaufnahme vor dem Bruchversuch zu sehen. Anhand des Kontrastes der Hellfeldaufnahme im ausgedünnten Bereich der Lamelle ist zu erkennen, dass sich im Bereich der Kerbe und ca. $600 \mathrm{~nm}$ entfernt von der Spitze der Kerbe jeweils kristalline Bereiche gebildet haben (vgl. Anhang C). Abbildung 4.15b zeigt die Hellfeldaufnahme nach dem ersten von zwei Verformungsschritten. Es ist zu erkennen, dass wie erwartet von der Spitze der Kerbe aus ein Riss nukleiert wird. Der Riss bildet sich innerhalb von ca. $2 \mathrm{~s}$, was im Vergleich zu den bisher gezeigten TiBruchversuchen ein deutlich schnellerer Prozess ist. Der Riss ist ca. $40 \mathrm{~nm}$ lang. Die Helligkeit im Bereich vor der Spitze der Kerbe (Markierung in Abbildung 4.15b) ist 


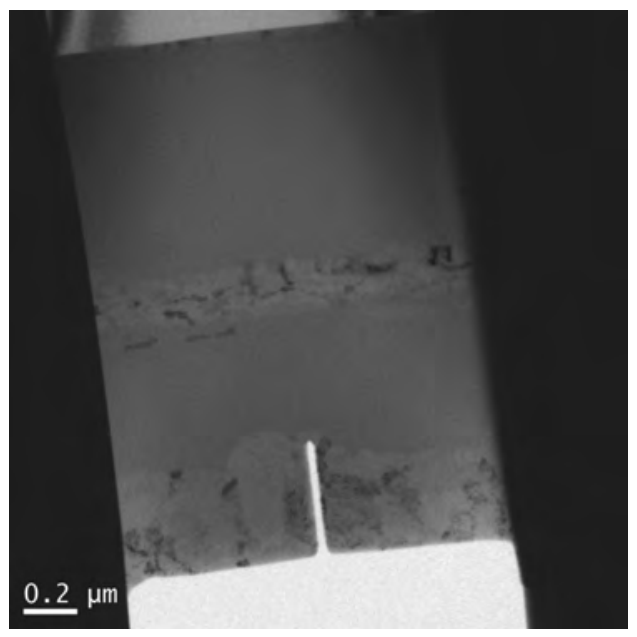

(a) BF vorher

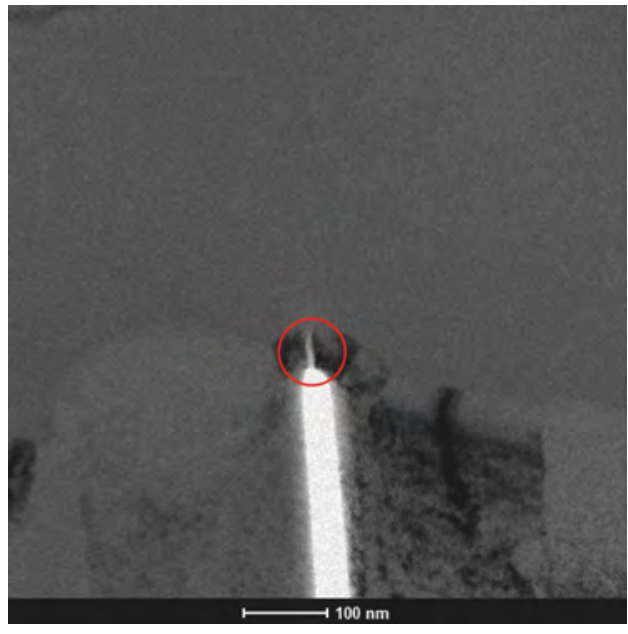

(b) Nach 1. Verformung

Abbildung 4.15: Bruchversuch einer amorphen $\mathrm{ZrO}_{2}$-Einzelschicht

geringer im Vergleich zum Bereich der Kerbe oder Bereichen, in denen kein Material durchstrahlt wird, aber auch höher im Vergleich zum umgebenden Material. Es ist daher vorstellbar, dass, wie für die Ti-Schichten, das Material hier lokal dünner und noch nicht komplett gerissen ist.

Während der Verformung können in den kristallinen Bereichen Biegekonturen beobachtet werden, die durch die mechanische Belastung kontinuierlich bewegt werden. Schnelle Kontrastwechsel, die mit Versetzungsaktivität in Verbindung stehen können, werden nicht beobachtet.

Während des zweiten Verformungsschrittes ist eindeutig zu erkennen, wie in dem helleren Bereich vor der Spitze der Kerbe ein Riss gebildet wird (Markierung in Abbildung 4.15b). Der Riss wächst im Verlauf der zweiten Verformung, bis es innerhalb weniger Einzelbilder zum katastrophalen Versagen der $\mathrm{ZrO}_{2}$-Schicht kommt. In Abbildung 4.16 a ist das letzte Einzelbild vor dem endgültigen Bruch zu sehen. Im Vergleich zur Konfiguration am Ende des ersten Verformungsschrittes (vgl. Abbildung 4.15b) fällt auf, dass der bereits verformte Bereich (Markierung in Abbildung 4.15b) gerissen ist. Ausgehend von der Spitze des Risses ist auch im zweiten Schritt ein Bereich helleren Kontrastes zu sehen (wie schon in Abbildung 4.15b). Entlang dieses helleren Bereichs (Markierung in Abbildung 4.16a versagt die Probe innerhalb von zwei Einzelbildern (ca. $0,2 \mathrm{~s}$ ) katastrophal.

In Abbildung 4.16c ist eine Übersichtsaufnahme des gesamten Risses zu sehen. Der Riss geht durch die gesamte $\mathrm{ZrO}_{2}$-Schicht und wird erst im Si-Substrat deutlich von der ursprünglich relativ geraden Ausbreitungsrichtung hin zu einer der Si-Säulen abgelenkt. Eine geringe Ablenkung ist im Bereich des kristallinen Bands im $\mathrm{ZrO}_{2} \mathrm{zu}$ erkennen. Die Oberfläche des Risses ist sehr glatt im Vergleich zu den Rissen im Ti. Erst in der Nähe zum Substrat ist die Rissoberfläche etwas rauer. Beim Durchgang durch den 


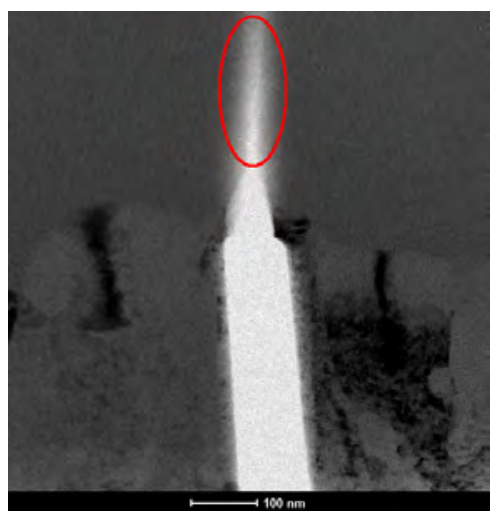

(a) Bild 429

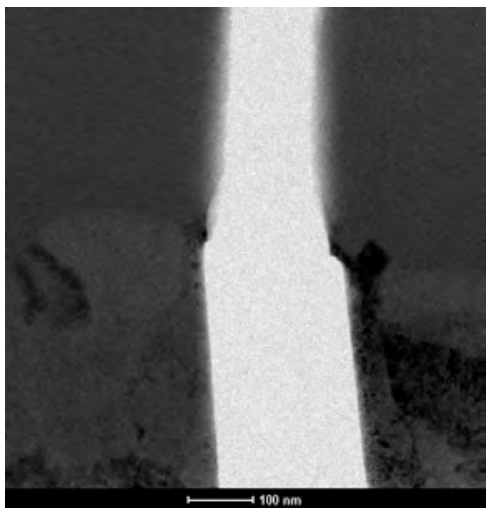

(b) Bild 431

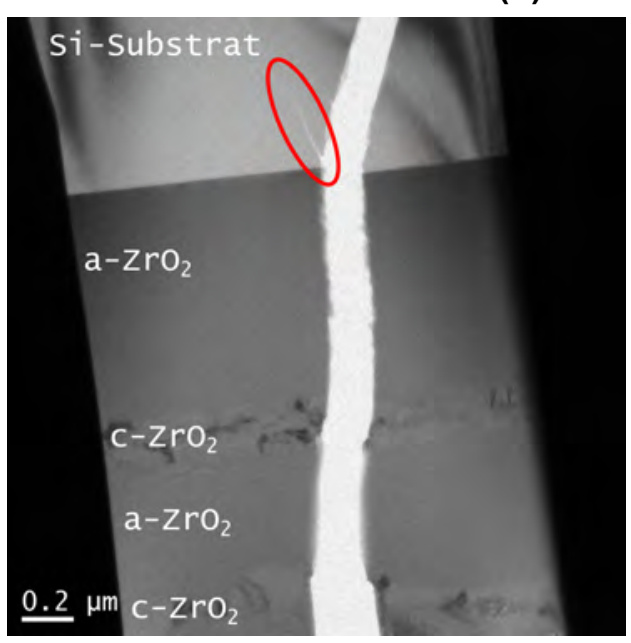

(c) Übersicht Riss

Abbildung 4.16: Bruchversuch einer amorphen $\mathrm{ZrO}_{2}$-Einzelschicht: (a) und (b) Zwei Einzelbilder des zweiten Verformungsschrittes, kurz vor und direkt nach dem katastrophalen Versagen der Schicht; (C) Übersichtsaufnahme des gesamten Risses am Ende des Bruchversuchs

kristallinen Bereich des $\mathrm{ZrO}_{2}$ in der Mitte der Lamelle ist keine Veränderung bezüglich der Rissoberfläche zu beobachten.

Zusätzlich ist im Bereich des Si-Substrates (Markierung in Abbildung 4.16c) ein zweiter Riss zu erkennen. Da jedoch dieser Bereich außerhalb des Bildausschnittes der Bildserien liegt, können keine weiteren Aussagen über den Ursprung dieses Risses oder seines Einflusses auf das Bruchverhalten getroffen werden.

\section{Kristalline Schicht}

Um eine kristalline $\mathrm{ZrO}_{2}$-Schicht untersuchen zu können, wird ein Teil der Probe im Ofen bei Umgebungsbedingungen ausgelagert. Die Probe wird für $20 \mathrm{~h}$ bei $600{ }^{\circ} \mathrm{C}$ gehalten. Die Aufheiz- und Abkühlphasen betragen jeweils $20 \mathrm{~h}$, um eine Delamination der Schicht vom unterliegenden Substrat aufgrund unterschiedlicher thermischer Ausdehnungskoeffizienten zu vermeiden. Nach der Auslagerung wird die Probe entsprechend 


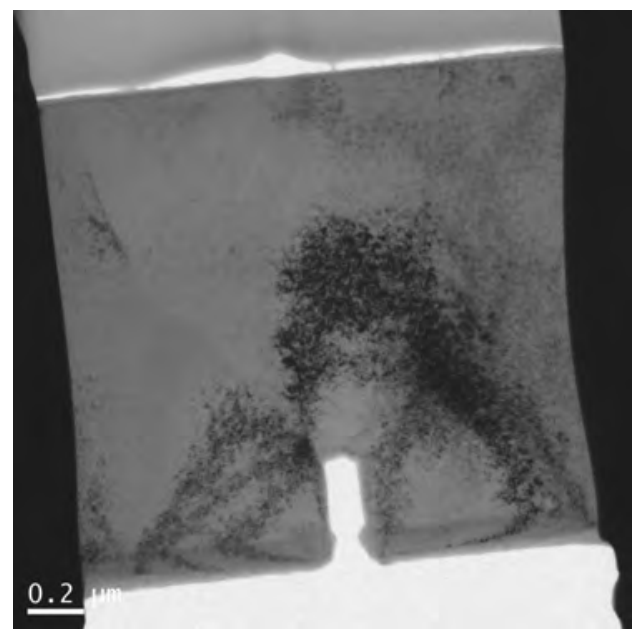

(a) BF vorher

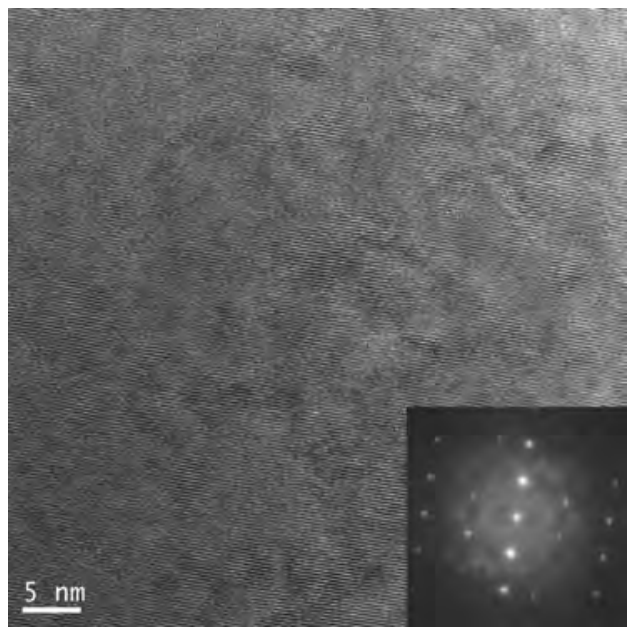

(b) HRTEM und FFT

Abbildung 4.17: Bruchversuch einer kristallinen $\mathrm{ZrO}_{2}$-Einzelschicht:(a) Hellfeldaufnahme vor dem Bruchversuch;(b) HRTEM-Aufnahme mit Fourier-Transformation

für die Bruchversuche präpariert.

In Abbildung 4.17a ist eine Hellfeldaufnahme einer ausgelagerten Lamelle zu sehen. Im Vergleich zur amorphen Schicht sind in der kristallinen Schicht deutlich Biegekonturen zu erkennen. Zusätzlich lässt die exemplarisch gezeigte HRTEM-Aufnahme und deren Fourier-Transformation in Abbildung 4.17b auf kristalline Strukturen schließen, sodass davon ausgegangen werden kann, dass es sich hier um eine vollständig kristalline Schicht handelt.

Der Bruchversuch ist in drei Verformungsschritte unterteilt. In den ersten beiden Schritten bewegen sich lediglich die Biegekonturen. Während des zweiten Schrittes wird durch die Verformung die Öffnungsweite der Kerbe etwas vergrößert, jedoch werden keine Kontrastwechsel wie in den Ti-Schichten oder eine Rissbildung beobachtet.

Im dritten Verformungsschritt bildet sich ein kleiner Riss an der Kerbe (siehe Markierung Abbildung 4.18a). Ausgehend von diesem Riss versagt die Schicht innerhalb der nächsten zwei Einzelbilder katastrophal. Während der Rissbildung bewegen sich, wie schon in den vorherigen Verformungsschritten, die Biegekonturen, jedoch ohne Anzeichen von Versetzungsaktivität. In Abbildung 4.18c ist eine Hellfeldübersichtsaufnahme des gesamten Risses gezeigt. Der Riss endet am Si-Substrat, welches im Gegensatz zum Bruchversuch der amorphen Schicht intakt bleibt.

\section{Energiefreisetzungsrate}

In Abbildung 4.19 sind die Risswiderstandskurven der amorphen und der kristallinen $\mathrm{ZrO}_{2}$-Schicht gezeigt. Für die amorphe Schicht (schwarze Symbole) konnte die Risswiderstandskurve in dem Verformungsschritt vor dem katastrophalen Versagen der 


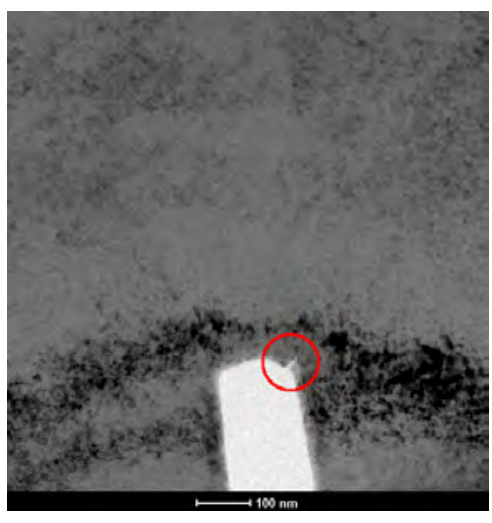

(a) Bild 173

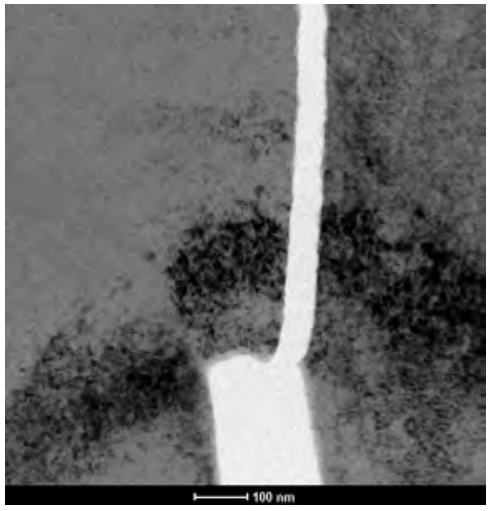

(b) Bild 175

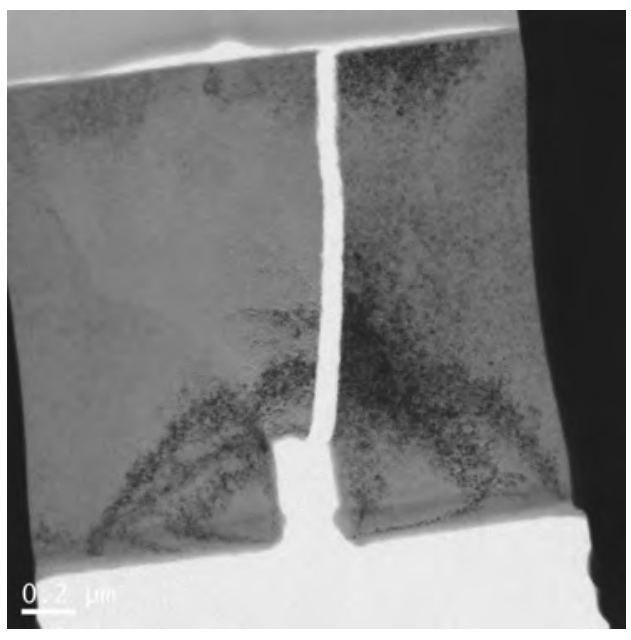

(c) Übersichtsaufnahme gesamter Riss

Abbildung 4.18: Bruchversuch einer kristallinen $\mathrm{ZrO}_{2}$-Einzelschicht: (a) und (b) Zwei Einzelbilder des zweiten Verformungsschrittes, kurz vor und direkt nach dem katastrophalen Versagen der Schicht; (C) Übersichtsaufnahme des gesamten Risses am Ende des Bruchversuchs

Schicht ausgewertet werden, da in diesem Schritt die Rissbildung stattfindet und hinreichendes Risswachstum vorhanden ist. Im Fall der kristallinen Schicht (blaue Kreise) finden die Rissbildung und das katastrophale Versagen der Schicht hingegen direkt nacheinander statt, sodass es hier zu keinem auswertbaren Risswachstum kommt. Die Risswiderstandskurve für den kristallinen Fall ist soweit wie möglich ausgewertet, muss aber als eine grobe Schätzung angesehen werden.

Anhand der kritischen Werte der Energiefreisetzungsrate $J_{I Q}$ wird die Bruchzähigkeit $K_{I Q}$ bei der Rissbildung berechnet (siehe Tabelle 4.5). Der Elastizitätsmodul wird für die amorphe und die kristalline Schicht mit $E_{\mathrm{ZrO}_{2}}=194,6 \mathrm{GPa}$ angenommen. 


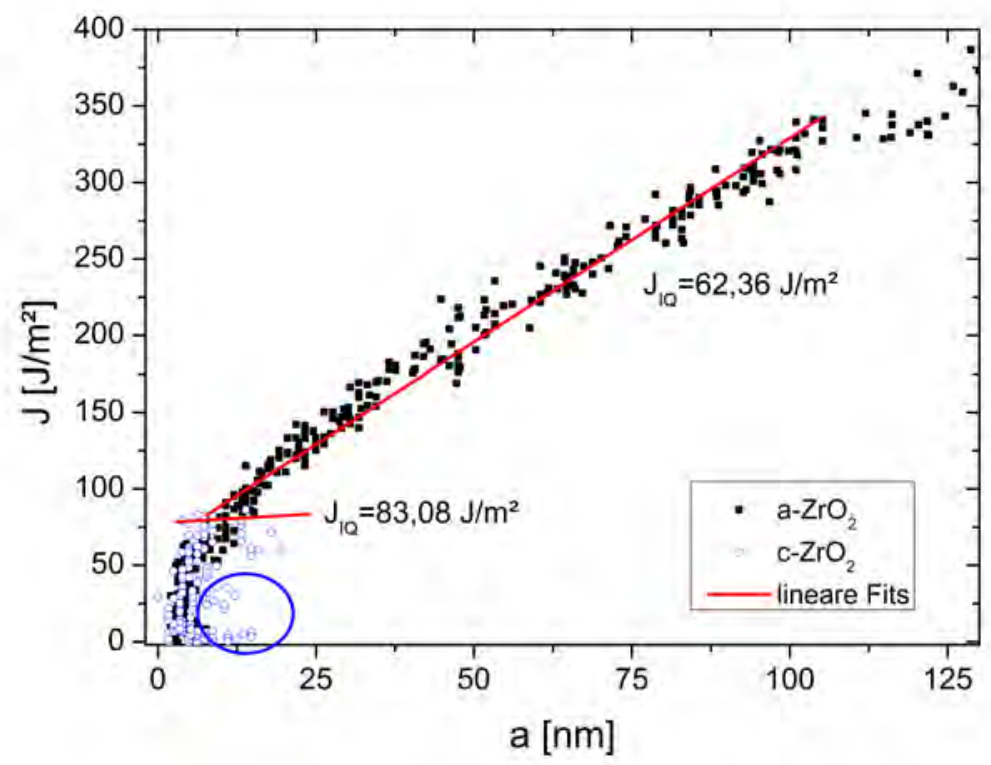

Abbildung 4.19: Energiefreisetzungsrate vs. Risslänge $\mathrm{ZrO}_{2}$-Einzelschichten; in schwarz amorphe Schicht, in blau kristalline Schicht, blau markierte Punkte sind Artefakte der automatischen Auswertung und werden für die lineare Anpassung zur Bestimmung der kritischen Bruchzähigkeit vernachlässigt

\begin{tabular}{lcc} 
& $J_{I Q}\left[\mathrm{~J} \mathrm{~m}^{-2}\right]$ & $K_{I Q}\left[\mathrm{MPa} \mathrm{m}^{1 / 2}\right]$ \\
\hline \hline amorph & $59,647(1257)$ & $3,364(169)$ \\
kristallin & $83,076(8615)$ & $4,021(945)$
\end{tabular}

Tabelle 4.5: Energiefreisetzungsrate und Bruchzähigkeit $\mathrm{ZrO}_{2}$-Einzelschichten 


\subsection{Bruchversuche entlang der Grenzflächen}

Im Folgenden werden die Bruchversuche der verschiedenen Multilagen-Proben in der Geometrie der Rissausbreitung entlang der Grenzflächen dargestellt. Zu jedem Schichtdickenverhältnis werden die Bruchversuche einzeln beschrieben und am Ende des jeweiligen Abschnittes eine Zusammenfassung der Ergebnisse angegeben. Es werden die Rissbildung sowie die Rissausbreitung analysiert. Nach der Darstellung der Ergebnisse der Bruchversuche der verschiedenen Ti-Schichtdicken werden am Ende dieses Abschnittes die Größen der plastischen Zonen sowie die kritischen Energiefreisetzungsraten in Abhängigkeit der Ti-Schichtdicke gezeigt.

Im Rahmen dieser Dissertation wurde eine zusätzliche Masterarbeit Mec15] realisiert, in der das Bruchverhalten der Multilagen entlang der Grenzflächen untersucht wurde. In dieser Masterarbeit wurden zusätzlich zu den Experimenten im Kontext der hier präsentierten Dissertation weitere Bruchversuche an den Ti100/ $\mathrm{ZrO}_{2} 100$ - und Ti10/ $\mathrm{ZrO}_{2}$ 100-Proben durchgeführt, um repräsentativere Daten zu erhalten. Außerdem wurden in der Masterarbeit die Ti50/ZrO 2 100-Proben entlang der Grenzflächen untersucht. An den entsprechenden Stellen wird auf die Ergebnisse der Masterarbeit verwiesen.

\subsection{1 $\mathrm{Ti100/Z \textrm {rO } _ { 2 } 1 0 0}$}

Ti100/ZrO 100 -Probe 1

In Abbildung 4.20a ist eine Übersichtsaufnahme des Bruchtests der Ti100/ZrO 2 100Probe 1 gezeigt. Die STM-Spitze wird von unten her an die Lamelle angenähert. In der Lamelle ist ein präparationsbedingter Ti-Tropfen zu sehen, welcher aufgrund der Lage oberhalb der Spitze der Kerbe den weiteren Bruchversuch nicht beeinflusst. Die Ti-Schichten sind jeweils die Schichten helleren Kontrastes und die $\mathrm{ZrO}_{2}$-Schichten entsprechend die dunkleren in einer Hellfeldabbildung. Die Spitze der Kerbe liegt innerhalb einer $\mathrm{ZrO}_{2}$-Schicht, was in höher vergrößerten Aufnahmen (hier nicht gezeigt) eindeutig festzustellen ist.

Der Bruchversuch wird in zwei Verformungsschritten durchgeführt. Die Abbildungen $4.20 \mathrm{~b}$ bis $4.20 \mathrm{e}$ zeigen verschiedene Momentaufnahmen während des ersten Verformungsschrittes. Die Belichtungszeit der Einzelaufnahmen der Bildserie beträgt 0,1s, sodass Abbildung 4.20b ca. 10 s nach Beginn des Bruchversuchs aufgenommen wurde. Nachdem der Kontakt zwischen den Si-Säulen und der STM-Spitze erreicht ist, ist eine Vergrößerung der Öffnungsweite der anfänglichen Kerbe zu beobachten. Aufgrund der gewählten Vergrößerungen können hier nur schwierig Kontrastveränderungen in den 100 nm-dicken Ti-Schichten erkannt werden, jedoch ist es möglich eine Größe der 


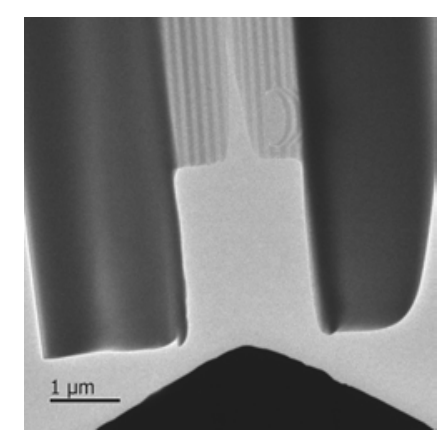

(a) Übersicht vorher

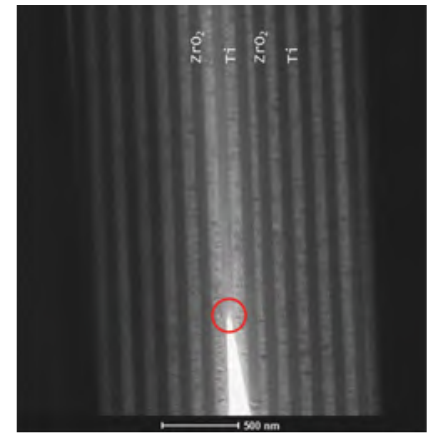

(b) BF Nr. 100

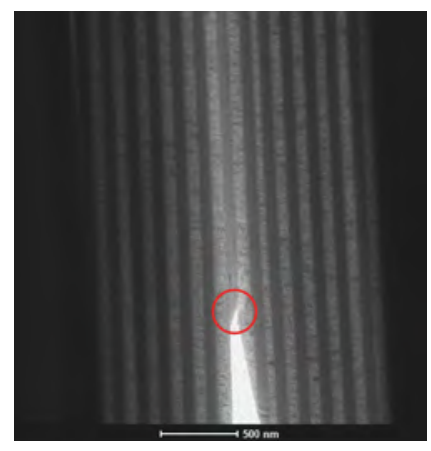

(c) BF Nr. 200

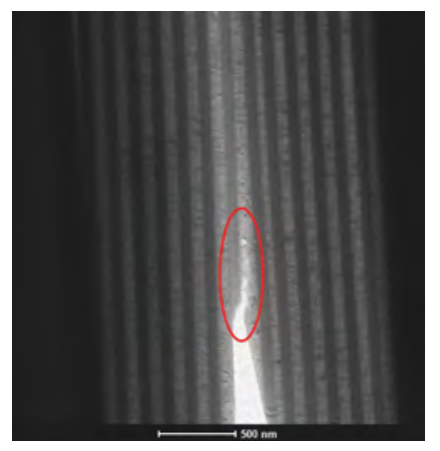

(d) BF Nr. 260

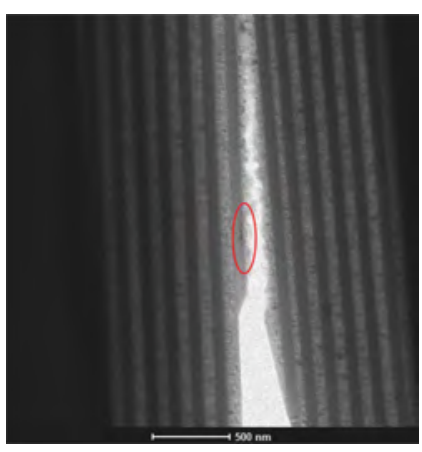

(e) BF Nr. 443

Abbildung 4.20: Bruchversuch Ti100/ZrO 100 entlang der Grenzflächen Probe 1 im ersten Verformungsschritt, Beschriftung der einzelnen Schichten in (b): Abbildungen (b) bis (e) zeigen verschiedene Momentaufnahmen während der Verformung

plastischen Zone zu bestimmen. In Abbildung 4.20b bildet sich der Riss innerhalb des $\mathrm{ZrO}_{2}$ ausgehend von der Spitze der Kerbe (siehe Markierung in Abbildung 4.20b). Mit fortschreitender Verformung bzw. Öffnung der Kerbe wächst der Riss weiter, wird jedoch direkt in Richtung der benachbarten Ti-Schicht auf der rechten Seite der Kerbe abgelenkt (Markierung in Abbildung 4.20c).

Abbildungen 4.20d und 4.20e zeigen das weitere Risswachstum innerhalb der Ti-Schicht im weiteren Verlauf des ersten Verformungsschrittes. Der Riss breitet sich, nachdem er die Ti-Schicht erreicht hat, in dieser über eine gesamte Distanz von ca. $a=1,4 \mu \mathrm{m}$ weiter aus. Während des Risswachstums innerhalb der Ti-Schicht befindet sich der Riss etwa in der Mitte der Ti-Schicht. Des Weiteren ist eine raue Rissoberfläche zu beobachten. Die Rauigkeit liegt hier im Bereich der Korngröße, die etwa der halben Schichtdicke entspricht.

Innerhalb der $\mathrm{ZrO}_{2}$-Schicht ist nur eine Biegekontur direkt in der Nähe der Spitze zu erkennen, die sich im Verlauf des ersten Verformungsschrittes bewegt und als Kontrast an der Grenzfläche zur gerissenen Ti-Schicht auch ohne weitere Verformung zu erkennen ist (Markierung in Abbildung 4.20e). Weitere Kontrastveränderungen, vergleichbar zu denen in den Ti-Schichten, können innerhalb der $\mathrm{ZrO}_{2}$-Schichten nicht festgestellt werden. 


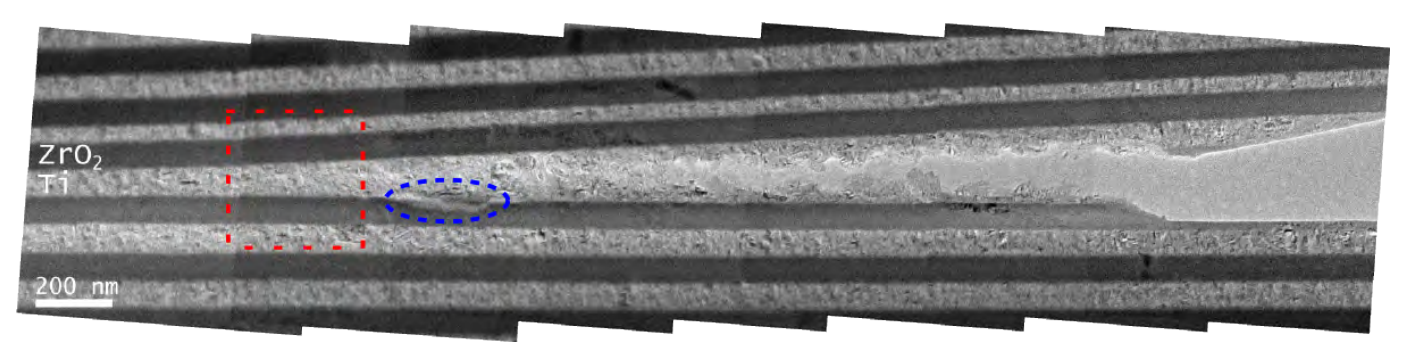

Abbildung 4.21: Übersicht Riss $\mathrm{Ti} 100 / \mathrm{ZrO}_{2} 100$ Probe 1 nach erstem Verformungsschritt

In Abbildung 4.21 ist eine zusammengesetzte Übersichtsaufnahme des Risses nach dem ersten Verformungsschritt gezeigt. Auch hier ist die raue Oberfläche des Risses in der Ti-Schicht eindeutig zu erkennen. Zusätzlich ist eine Fehlstelle in der $\mathrm{ZrO}_{2}$-Schicht, in der ursprünglich die Spitze der Kerbe lag, zu beobachten (blaue Markierung in Abbildung 4.21). In diesem Bereich ist die Ti-Schicht noch nicht gerissen, sondern nur stark gedehnt. Die Spitze des Risses liegt etwa am rechten Rand der blauen Markierung. Durch die Verformung wird im Bereich der blauen Markierung ein Teil der $\mathrm{ZrO}_{2}$-Schicht abgelöst. Diese Verformung steht nicht in Zusammenhang mit der FIBinduzierten Kristallisation der $\mathrm{ZrO}_{2}$-Schicht, da vor Versuchsbeginn in diesem Bereich keine Kontraste im $\mathrm{ZrO}_{2}$ beobachtet werden konnten, die einen Hinweis auf eine kristalline $\mathrm{ZrO}_{2}$-Schicht geben würden.

Für den zweiten Verformungsschritt wird eine höhere Vergrößerung gewählt und der Bereich der Probe vor der angenommenen Spitze des Risses genauer beobachtet. In Abbildung 4.21 ist der gewählte Bildausschnitt für den zweiten Schritt markiert (rote Markierung). In den Abbildungen 4.22a bis 4.22c sind Momentaufnahmen während des zweiten Verformungsschrittes gezeigt. Die rote Markierung in Abbildung 4.22a zeigt die bereits gerissene Ti-Schicht, in der der Riss auch im zweiten Verformungsschritt zunächst weiter wächst. Die $\mathrm{ZrO}_{2}$-Schicht links von der gerissenen Ti-Schicht ist ebenfalls schon geschädigt. Zusätzlich ist zu erkennen, dass sich in der nächsten Ti-Schicht links von dem Riss (blaue Markierung in Abbildung 4.22a) erste Hohlräume bilden. Im Verlauf der weiteren Verformung (siehe Abbildungen 4.22b und 4.22c) ist zu erkennen, dass der Riss die Ti-Schicht gewechselt hat. D.h. von der ursprünglich gerissenen Ti-Schicht (rote Markierung in Abbildung 4.22a) aus kreuzt der Riss die amorphe $\mathrm{ZrO}_{2}$-Schicht, wird in der links angrenzenden Ti-Schicht renukleiert und breitet sich dort aus. Dieser Übergang von Ti über $\mathrm{ZrO}_{2}$ wieder nach Ti wird im Folgenden als Scherband bezeichnet, da die Verformung des nanoskaligen $\mathrm{ZrO}_{2}$ mittels Scherbändern erfolgt Kno12. In Abbildung 4.22b ist anhand des hellen Kontrastes in der Ti-Schicht links des Risses deutlich zu erkennen, dass hier durch die Verformung die Ti-Schicht dünner wird und bereits stellenweise gerissen ist. Zu Beginn des zweiten Verformungsschrittes sind in den involvierten Ti-Schichten ausgeprägte Versetzungsaktivitäten zu erkennen. Es gibt im gesamten Bildausschnitt (ca. $400 \mathrm{~nm} \times 400 \mathrm{~nm}$ ) sehr viele Kontrastwechsel. Zu dem 


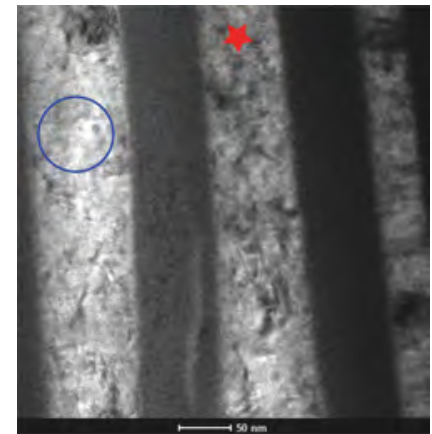

(a) BF Nr. 115

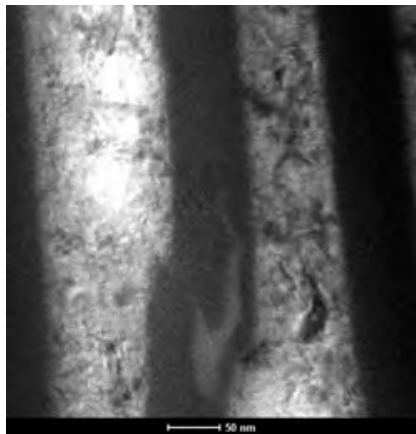

(b) BF Nr. 190

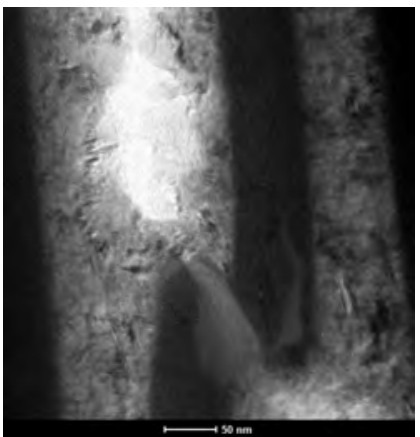

(c) BF Nr. 430

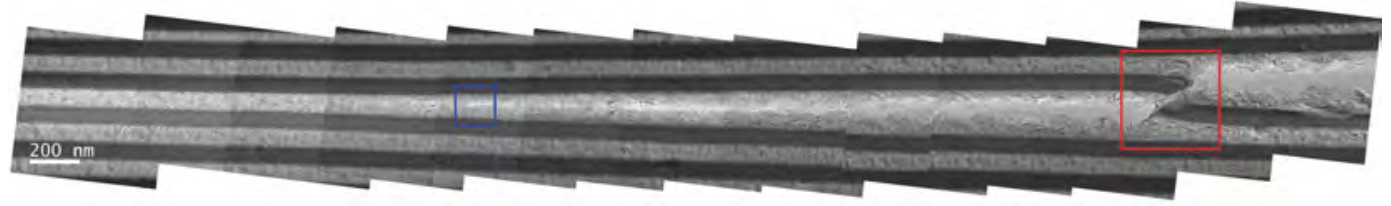

(d) BF-Übersicht Riss

Abbildung 4.22: Bruchversuch Ti100/ZrO 100 entlang der Grenzflächen Probe 1 zweiter Verformungsschritt: Abbildungen (a) bis (c) zeigen die Ausbildung des Scherbandes und den Wechsel der Rissausbreitung in die nächte Ti-Schicht

Zeitpunkt, an dem sich das Scherband in der $\mathrm{ZrO}_{2}$-Schicht bildet, verringert sich die Versetzungsaktivität in der ursprünglich gerissenen Ti-Schicht signifikant. Umso weiter der neu gebildete Riss aus dem Bildausschnitt heraus wächst, desto geringer wird auch die Versetzungsaktivität in der Ti-Schicht. Lediglich direkt an dem Scherband ist auch gegen Ende der Verformung Versetzungsaktivität zu beobachten.

Nachdem sich der Riss an den Hohlräumen gebildet hat, wächst er über eine Distanz von ca. 2,6 $\mathrm{m}$ in dieser Ti-Schicht ohne noch einmal die Ti-Schicht zu wechseln. In Abbildung $4.22 \mathrm{~d}$ ist eine zusammengesetzte Übersichtsaufnahme des Risses nach der zweiten Verformung zu sehen. Die Stelle des Scherbandes und die Rissspitze sind markiert. Das Rissverhalten nach dem Scherband ist vergleichbar mit dem Verhalten im ersten Verformungsschritt. Der Riss breitet sich etwa in der Mitte der Ti-Schicht aus und auch die Oberfläche des Risses ist ähnlich rau.

\section{Ti100/ZrO $100-$ Probe 2}

Als zweites Beispiel eines Bruchversuchs der Ti100/ $\mathrm{ZrO}_{2} 100$-Proben wird das Experiment mit der Lamelle in Abbildung 4.23a beschrieben. In den Abbildungen 4.23a und $4.23 \mathrm{~b}$ sind Hellfeldaufnahmen der gesamten Lamelle und in höherer Vergrößerung die Position der Kerbe gezeigt. Die Spitze der Kerbe liegt hier eindeutig in einer einzelnen Ti-Schicht.

Dieser Bruchversuch ist in sechs einzelne Verformungsschritte unterteilt. Im ersten Schritt bewegen, sich nachdem der Kontakt zwischen STM-Spitze und Lamelle aufge- 


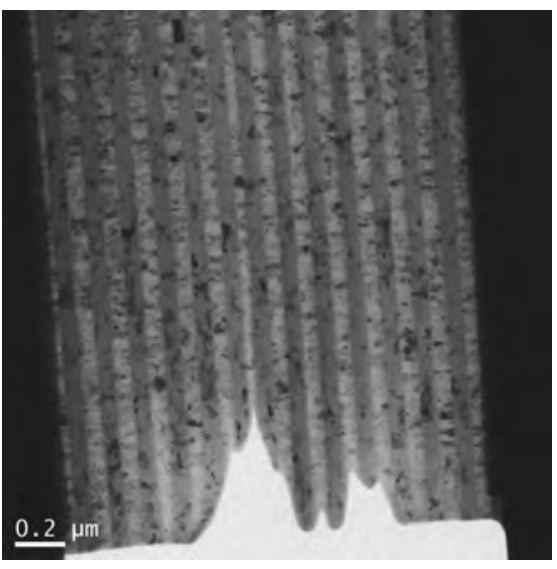

(a) BF vorher

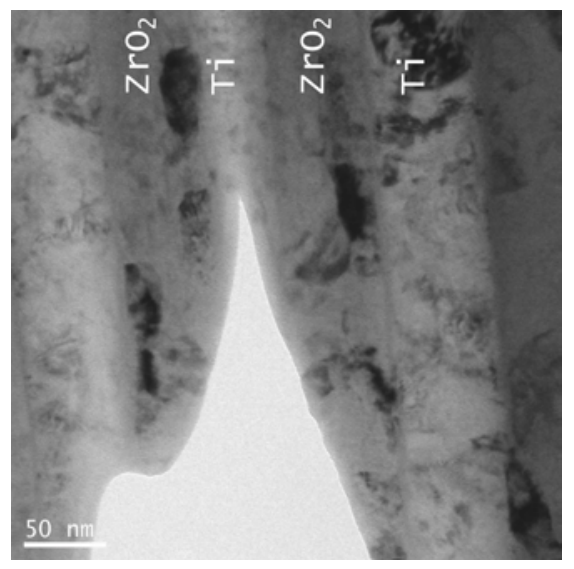

(b) BF-Kerbe

Abbildung 4.23: Bruchversuch Ti100/ZrO 100 entlang der Grenzflächen Probe 2

baut ist, Biegekonturen über die gesamte Lamelle und die Öffnungsweite der Kerbe wird vergrößert. Dem hingegen wird während des zweiten Verformungsschrittes der Riss an der Spitze der Kerbe gebildet. Der Riss wächst mit weiterer Verformung innerhalb der Ti-Schicht. Am Ende des zweiten Schrittes ist der Riss ca. $150 \mathrm{~nm}$ lang. Die Rissoberfläche sowie die mittige Lage innerhalb der Ti-Schicht sind vergleichbar mit den bisher gezeigten Beobachtungen.

Während des zweiten Verformungsschrittes bewegen sich weiterhin Biegekonturen sowohl in den Ti- als auch in den $\mathrm{ZrO}_{2}$-Schichten. Bevor der Riss gebildet wird, gibt es vereinzelt Versetzungsaktivität in der Nähe der Spitze der Kerbe. Mit der tatsächlichen Bildung des Risses wird die Aktivität erhöht und hält an, während der Riss wächst. In den letzten Einzelbildern dieser Bildsequenz sind einige Relaxationsprozesse in Form von einiger Versetzungsaktivität zu beobachten.

In den folgenden Bildserien (hier nicht gezeigt) ist mit einer höheren Vergrößerung das Verhalten in der Nähe der Rissspitze dokumentiert. In diesen Verformungssegmenten ist das Ausbreiten des Risses in der bereits gerissenen Ti-Schicht zu beobachten. Der Bruch der Ti-Schicht geht mit einer konzentrierten Versetzungsaktivität innerhalb dieser Ti-Schicht einher. In den anderen Ti-Schichten werden weiterhin Versetzungsaktivitäten verzeichnet, die aber deutlich weniger stark ausgeprägt sind im Vergleich zur Schicht mit dem Riss.

In Abbildung 4.24 ist eine zusammengesetzte Aufnahme des Risses am Ende des Versuchs zu sehen. Der Riss ist ausgehend von der Kerbe über eine Länge von ca. $6 \mu \mathrm{m}$ gewachsen. Über die gesamte Länge breitet sich der Riss innerhalb der einen Ti-Schicht aus, in der er auch gebildet wird. Scherbänder wie im vorherigen Experiment werden nicht beobachtet. 


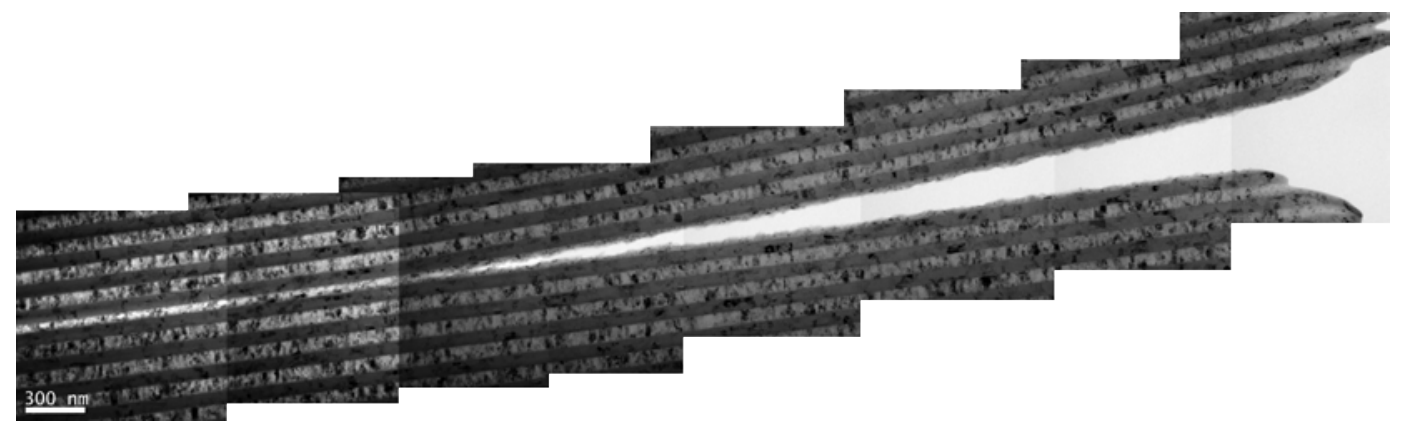

Abbildung 4.24: Übersicht finaler Riss Ti100/ZrO 100 Probe 2

\section{Ti100/ZrO 2 100-Probe 3 [Mec15]}

Der Bruchversuch einer weiteren Probe ist in sechs Verformungsschritte unterteilt. Die Position der Kerbe liegt sowohl in einer $\mathrm{ZrO}_{2}$ - als auch in einer Ti-Schicht, die tatsächliche Spitze der Kerbe jedoch eindeutig im $\mathrm{ZrO}_{2}$. Im ersten Schritt öffnet sich die Kerbe und Biegekonturen bewegen sich über die Lamelle.

Im zweiten Schritt öffnet sich die Kerbe weiter und an der Spitze der Kerbe bilden sich zwei Risse. Beide Risse werden in der $\mathrm{ZrO}_{2}$-Schicht gebildet, wobei der blau markierte Riss in Abbildung 4.25a zuerst beobachtet wird, aber nicht weiter wächst. Der zweite Riss, der auch im $\mathrm{ZrO}_{2}$ nukleiert wird, wächst wie der von Probe 1 innerhalb des dritten Verformungsschrittes direkt in die benachbarte Ti-Schicht. In Abbildung 4.25a ist die Konfiguration nach dem dritten Verformungsschritt zu sehen. Der Riss hat sich in der Mitte der Ti-Schicht ausgebreitet. Während des Risswachstums sind Versetzungsaktivitäten in den benachbarten Ti-Schichten zu erkennen, wobei tendenziell eine höhere Aktivität in der Ti-Schicht links von der gerissenen Schicht zu beobachten ist.

In dem rot markierten Bereich in Abbildung 4.25a ist zusätzlich anhand des helleren Kontrastes zu erkennen, dass die $\mathrm{ZrO}_{2}$-Schicht hier lokal dünner geworden ist. Der Pfeil markiert eine Position in der Ti-Schicht links von der gerissenen Schicht, an der sich ein erster Hohlraum in der noch intakten Ti-Schicht gebildet hat.

Im vierten Verformungsschritt wird im Bereich der roten Markierung die Verformung in höherer Vergrößerung beobachtet. Während der Verformung ist zu erkennen, wie sich ein Scherband in der $\mathrm{ZrO}_{2}$-Schicht bildet. In Abbildung 4.25b ist das letzte Einzelbild der Bildserie des vierten Schrittes gezeigt. Die Verformung ist von einer massiven Versetzungsaktivität in den beiden beteiligten Ti-Schichten begleitet. Auch in der anderen Ti-Schicht innerhalb des Bildausschnittes ist Aktivität zu beobachten, jedoch deutlich weniger. Das tatsächliche Kreuzen der $\mathrm{ZrO}_{2}$-Schicht konnte nicht live beobachtet werden. In Abbildung 4.25c ist das Scherband kurz nach dem Ende des vierten Schrittes zu sehen. Durch Relaxationsprozesse muss sich das Scherband endgültig so gebildet haben, sodass die $\mathrm{ZrO}_{2}$-Schicht gerissen ist.

In den folgenden zwei Verformungsschritten wächst der Riss, wie schon in anderen 


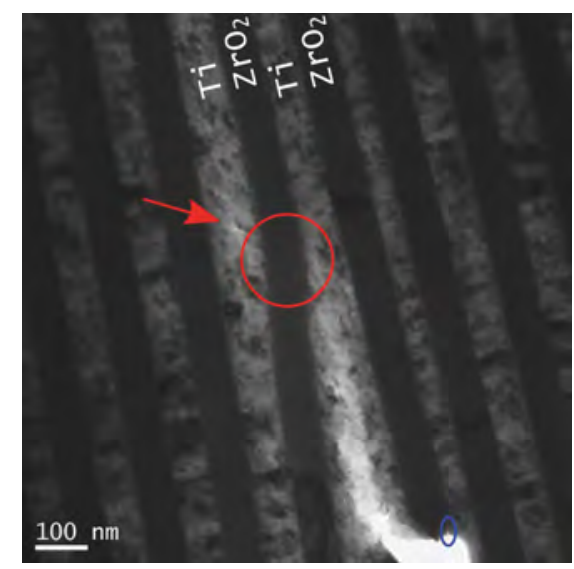

(a) nach 3. Schritt

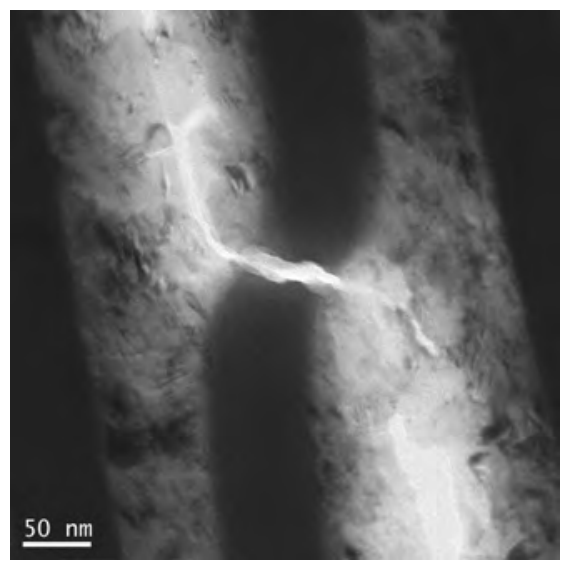

(c) Scherband Anfang 5. Schritt

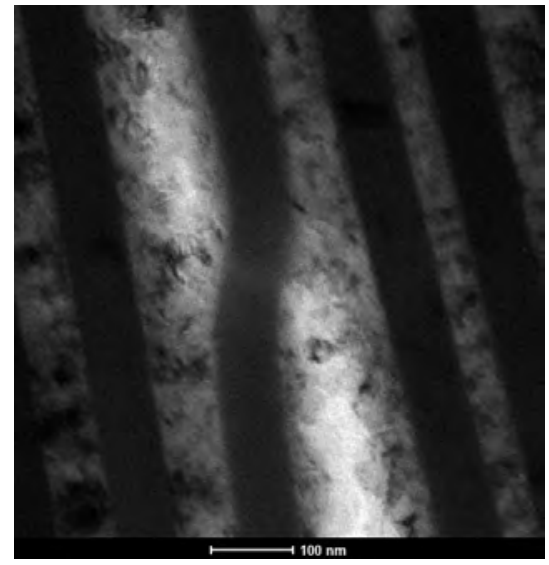

(b) Scherband Ende 4. Schritt

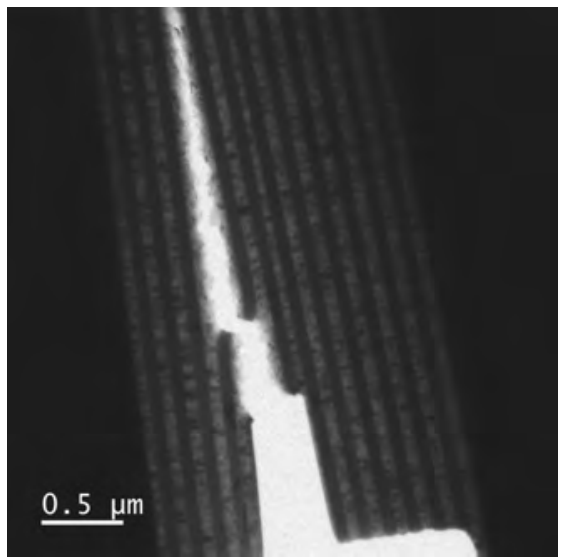

(d) BF-Übersicht Riss Ende

Abbildung 4.25: Bruchversuch Ti100/ZrO 100 entlang der Grenzflächen Probe 3

Bruchversuchen beobachtet, in der Ti-Schicht. Das Rissverhalten folgt dem schon bekannten Verhalten - ein Riss in der Mitte der Ti-Schicht und eine raue Rissoberfläche. Der gesamte Riss am Ende des Versuchs ist in Abbildung 4.25d gezeigt. Nach dem Scherband ist der Riss noch über mehr als $2 \mu \mathrm{m}$ im Ti gewachsen.

\section{Ti100/ZrO 100 -Probe 4}

In Abbildung 4.26 ist jeweils eine Hellfeldübersichtsaufnahme vor und nach dem Bruchversuch der Probe zu sehen. Die Spitze der Kerbe liegt hier ursprünglich in sowohl einer $\mathrm{ZrO}_{2}$ als auch einer Ti-Schicht. Der Bruchversuch ist in vier Verformungssegmente unterteilt. Im ersten Segment wird die Öffnungsweite der Kerbe größer und im zweiten Schritt bildet sich ein Riss an der Spitze der Kerbe, jedoch in der $\mathrm{ZrO}_{2}$-Schicht. Im dritten Verformungsschritt breitet sich der Riss von der $\mathrm{ZrO}_{2}$-Schicht hin zur Ti-Schicht links von der Kerbe aus. Zusätzlich ist jedoch in der vierten $\mathrm{ZrO}_{2}$-Schicht rechts von der Kerbe ein Riss zu beobachten. Im letzten Verformungsschritt wächst sowohl der Riss im Ti als auch im $\mathrm{ZrO}_{2}$. Innerhalb der Ti-Schicht ist eine Risslänge von ca. $200 \mathrm{~nm}$ 


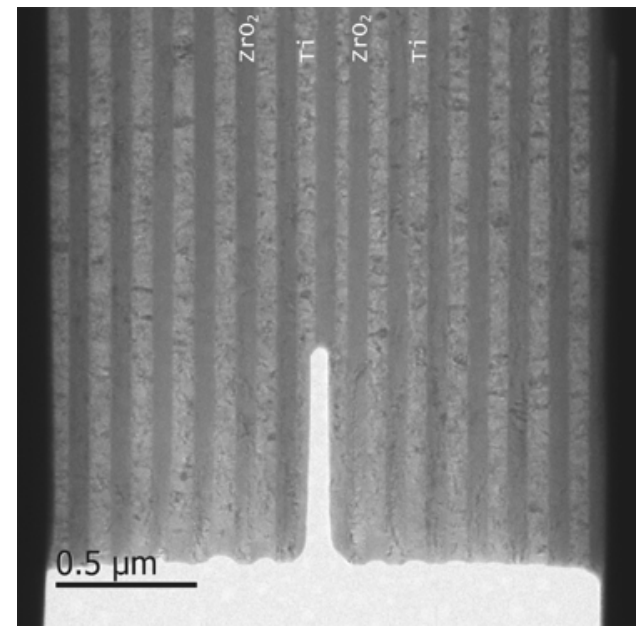

(a) BF vorher

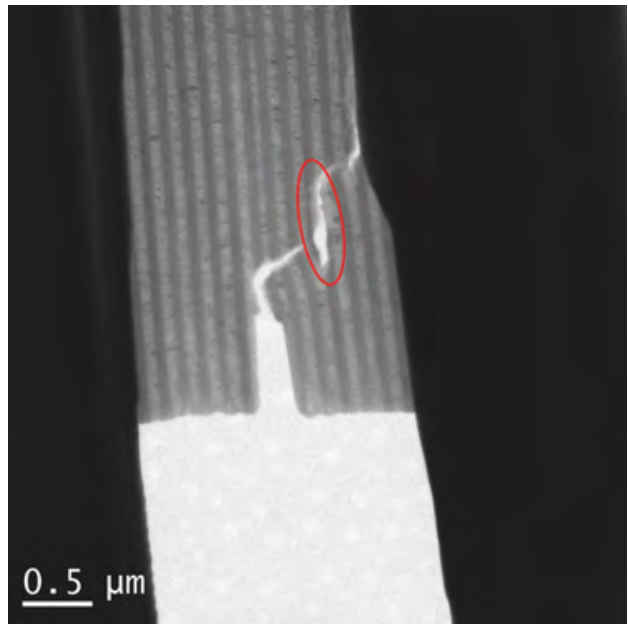

(b) BF nach dem Versuch

Abbildung 4.26: Bruchversuch Ti100/ZrO 100 entlang der Grenzflächen Probe 4

$\mathrm{zu}$ verzeichnen, bis der Ti-Riss nach rechts hin zum Riss im $\mathrm{ZrO}_{2}$ abgelenkt wird, wobei er mehrere $\mathrm{ZrO}_{2}$ - als auch Ti-Schichten kreuzt. Die finale Konfiguration dieses Bruchversuchs ist in Abbildung 4.26b zu sehen. Das Rissverhalten dieser Probe steht in Verbindung mit einer nicht hinreichend guten Ausrichtung der STM-Spitze relativ zu den Si-Säulen der Lamelle. Die Spitze ist verdreht und/oder verkippt justiert, sodass nicht gleichzeitig der Kontakt zu beiden Si-Säulen erreicht wird und im weiteren Verlauf eine asymmetrische Verformung anzunehmen ist (vgl. 4.2). Diese asymmetrische Verformung ist auch an den unterschiedlich stark verbogenen Si-Säulen zu erkennen.

Dieser Versuch ist aus oben genannten Gründen nicht vollständig vergleichbar mit den anderen hier gezeigten Bruchversuchen für dieses Schichtdickenverhältnis. Das Verhalten bei der Rissbildung und des anfänglichen Risswachstums kann jedoch weiter für die Diskussion des Bruchverhaltens verwendet werden.

\section{Ti100/ZrO 2100 -Probe 5}

Als letztes Beispiel einer Ti100/ZrO 100 -Probe wird hier ein Bruchversuch im SEM beschrieben. Dieses Experiment ist mit dem in-situ Aufbau des SEM (siehe Abschnitt A und Kap11]) durchgeführt worden, jedoch in diesem Fall ohne einen Kraftsensor. D.h. die Lamelle wird gegen eine gebrauchte Spitze eines Mikromanipulators, die auch eine Keilform wie die STM-Spitze hat, gedrückt. Dieser Bruchversuch ist nicht in verschiedene Verformungsschritte unterteilt.

Die Lamelle wird kontinuierlich gegen die Spitze gedrückt und es wird ein Riss gebildet. In Abbildung 4.27a ist die SEM-Aufnahme nach dem Bruchversuch zu sehen, jedoch bleibt nur zu erahnen, in welcher Schicht sich der Riss ausgebreitet hat. Die gerissene Lamelle wird nach dem Bruchversuch im SEM im TEM analysiert, jedoch 


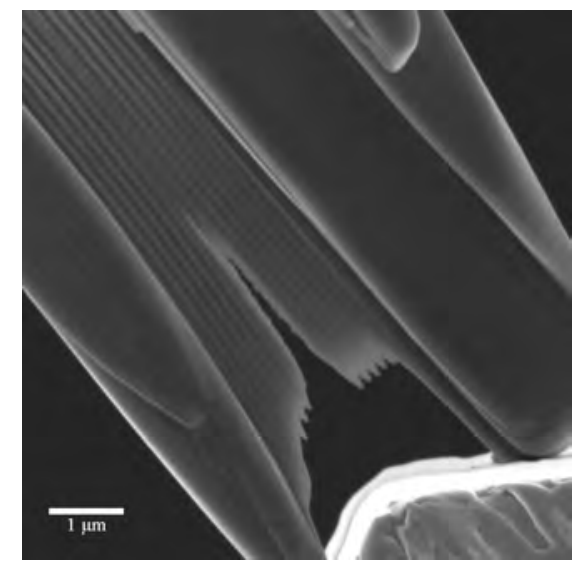

(a) SEM-Aufnahme nach Versuch

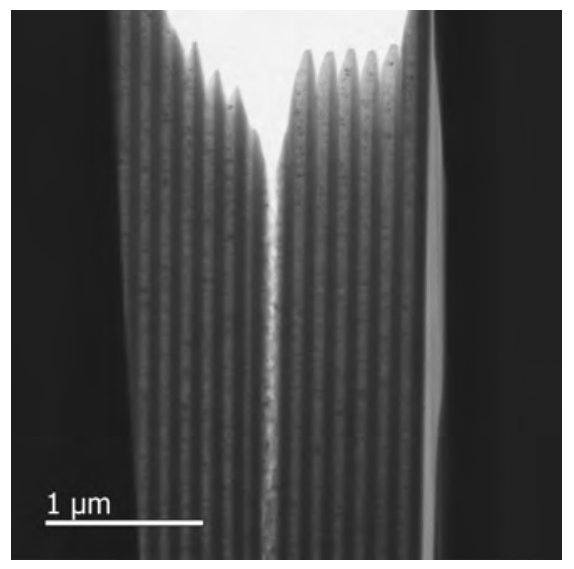

(b) BF-TEM ohne STM-Spitze

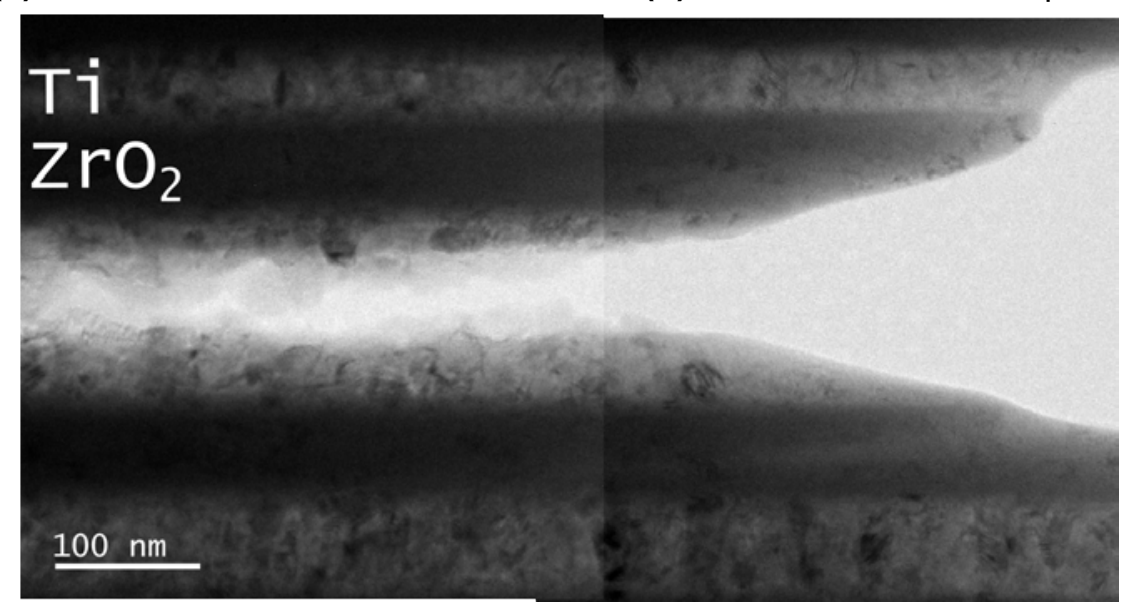

(c) BF des Risses im TEM

Abbildung 4.27: Bruchversuch Ti100/ZrO 100 entlang der Grenzflächen im SEM Probe 5

ohne die STM-Spitze wie in den in-situ TEM-Bruchversuchen, sodass der Riss teilweise geschlossen ist.

In Abbildung 4.27b ist eine Hellfeldübersichtsaufnahme des Risses und in Abbildung $4.27 \mathrm{c}$ eine zusammengesetzte Aufnahme in höherer Vergrößerung zu sehen. Anhand dieser Aufnahmen ist zu erkennen, dass die Spitze der Kerbe zu Beginn des Bruchversuchs in einer Ti-Schicht liegt. Ausgehend von der Kerbe bildet sich so der Riss in der Ti-Schicht und breitet sich in dieser über mehr als $2 \mu \mathrm{m}$ aus. Die zusammengesetzte TEM-Aufnahme der ersten $300 \mathrm{~nm}$ des Risses nach der Kerbe bestätigt die Beobachtungen der in-situ TEM-Bruchversuche. Die Risse für die $100 \mathrm{~nm}$ dicken Ti-Schichten liegen innerhalb der Ti-Schichten. Weiter entfernt von der Kerbe können keine TEMAufnahmen ausgewertet werden, da die Rissoberflächen hier überlappen und keine konkreten Erkenntnisse zulassen. Dieser Versuch kann ebenfalls nicht bezüglich der Größe der plastischen Zone oder der kritischen Energiefreisetzungsrate analysiert werden. 


\section{Zusammenfassung $\mathrm{Ti1} 100 / \mathrm{ZrO}_{2} 100$}

Zusammengefasst zeigen alle Bruchversuche der Ti100/ZrO 2100 -Probenserie ein vergleichbares Verhalten. In allen Experimenten kann die Rissbildung an der Spitze der Kerbe beobachtet werden, wobei es keinen Unterschied macht, ob die Kerbe in einer $\mathrm{ZrO}_{2}$ - oder einer Ti-Schicht endet. Nach der Rissbildung kann dieser kontrolliert unter der fortlaufenden Verformung wachsen. Hier ist zu unterscheiden, ob der Riss im $\mathrm{ZrO}_{2}$ oder Ti gebildet wird. Einmal in einer Ti-Schicht gebildet, bleibt er für größere Risslängen auch in der Ti-Schicht. Wenn der Riss im $\mathrm{ZrO}_{2}$ gebildet wird (vgl. Probe 1), bewegt er sich direkt zur benachbarten Ti-Schicht, um dann in dieser Ti-Schicht weiter zu wachsen. In zwei der fünf Versuche konnte das Kreuzen einer $\mathrm{ZrO}_{2}$-Schicht im Verlauf des Risswachstums beobachtet werden. Dieses Kreuzen wird mit einer Scherbandbildung in der $\mathrm{ZrO}_{2}$-Schicht in Verbindung gebracht und steht nicht im Zusammenhang mit einer Kristallisation der $\mathrm{ZrO}_{2}$-Schicht im Bereich des Scherbandes. 


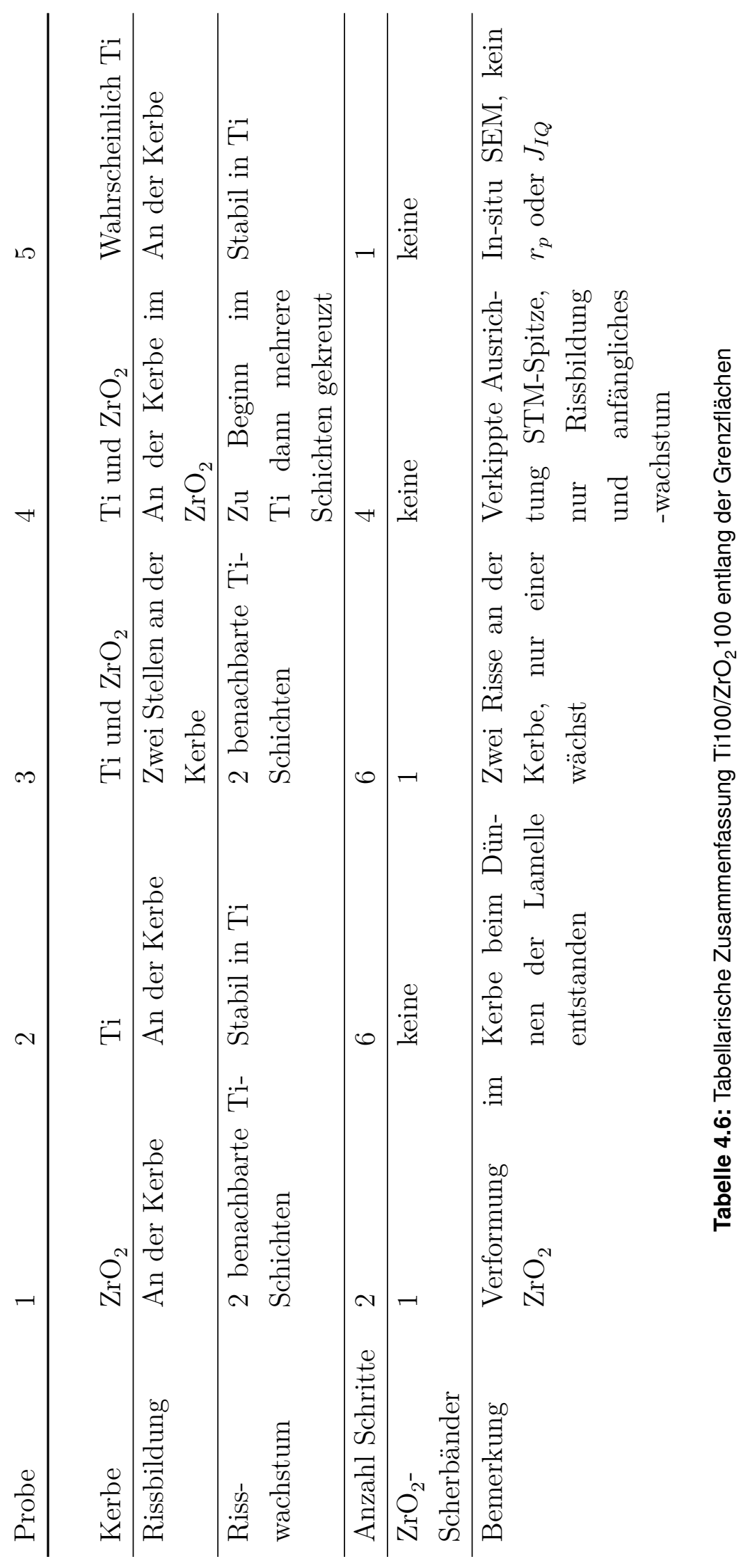




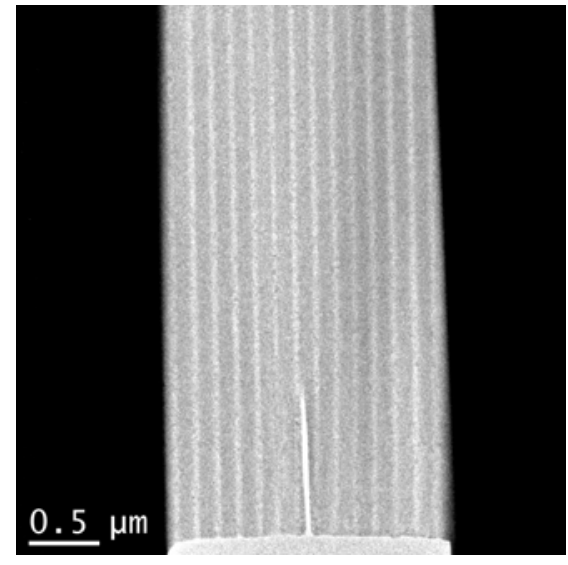

(a) TEM-Aufnahme vorher

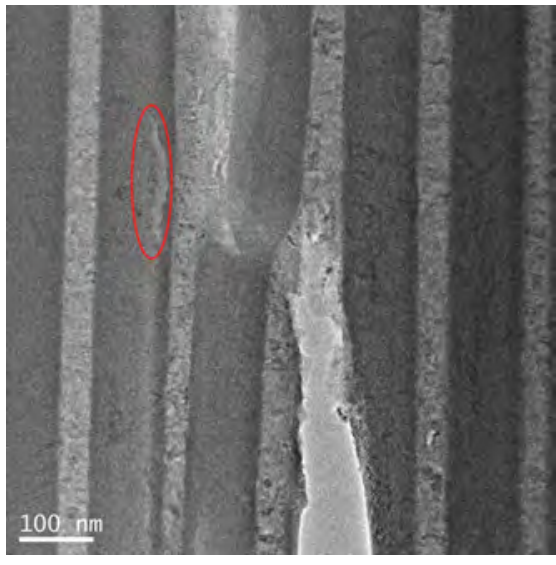

(b) nach 2. Verformung

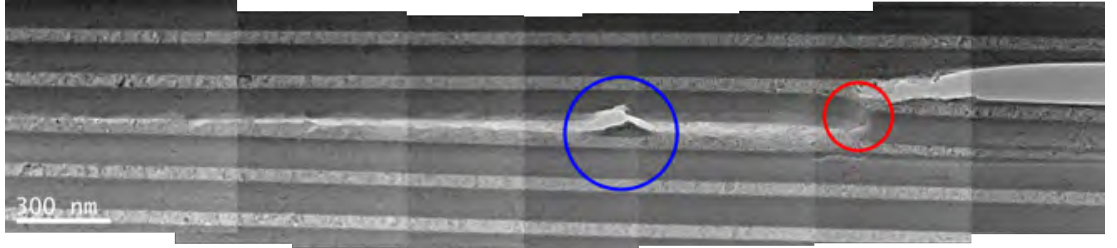

(c) Übersicht Riss im TEM nach zweiter Verformung zweiter Bruchversuch

Abbildung 4.28: Bruchversuch Ti50/ZrO $\mathrm{Z}_{2} 100$ entlang der Grenzflächen Probe 5

\subsection{2 $\mathrm{Ti50} / \mathrm{ZrO}_{2} 100[\mathrm{Mec} 15]$}

Dieses Schichtdickenverhältnis ist in der Masterarbeit [Mec15], die im Rahmen dieser Dissertation betreut wurde, untersucht worden. An dieser Stelle wird ein Bruchversuch (Probe 5 in [Mec15]) exemplarisch, ausführlich dargestellt und ausgewertet. Im Rahmen der Masterarbeit wurden sechs Bruchversuche durchgeführt und ausgewertet. Die Ergebnisse aller Bruchversuche an den Ti50/ $\mathrm{ZrO}_{2} 100$-Proben werden im Anschluss an die Darstellung eines Bruchversuchs in Tabellenform zusammengefasst und im weiteren Verlauf der Dissertation zur Analyse und Interpretation des Bruchverhaltens verwendet.

\section{Ti50/ZrO $100-$ Probe 5}

In Abbildung 4.28a ist eine Übersichtsaufnahme einer Ti50/ $\mathrm{ZrO}_{2} 100$-Probe $\mathrm{zu}$ sehen. Die Spitze der Kerbe liegt in diesem Fall in einer $\mathrm{ZrO}_{2}$-Schicht. Dieser Bruchversuch ist in vier Verformungsschritte unterteilt. Im zweiten Schritt wird der Riss ausgehend von der Spitze der Kerbe in der $\mathrm{ZrO}_{2}$-Schicht gebildet. Der Riss orientiert sich im Verlauf des zweiten Schrittes in Richtung der am nächsten liegenden Ti-Schicht auf der linken Seite der Kerbe. Der verformte Bereich erstreckt sich über ca. $150 \mathrm{~nm}$. Dieser Schritt wird für die Auswertung der Energiefreisetzungsrate genutzt.

In dem folgenden dritten Schritt, der bei höherer Vergrößerung aufgenommen wird, ist 
kein signifikantes Risswachstum zu beobachten. Jedoch kann die Aktivität von Versetzungen bis ca. $280 \mathrm{~nm}$ vor der Rissspitze beobachtet werden. Am Ende dieses Verformungsschrittes muss die STM-Spitze den Kontakt mit den Si-Säulen verloren haben, da im vierten Verformungsschritt keine weitere Verformung erreicht werden kann.

Mit einer besser ausgerichteten STM-Spitze wird ein zweiter Bruchversuch durchgeführt. Diese Verformungsschritte werden nicht quantitativ ausgewertet, da sie nicht vergleichbar zu den anderen Bruchversuchen sind. Der Rissverlauf kann jedoch weiter verwendet werden. Die Rissspitze des zuvor gebildeten Risses fungiert als neue potentielle Stelle der Rissbildung bzw. des Risswachstums. In diesem zweiten Bruchversuch werden wieder drei Verformungsschritte realisiert. Im ersten Schritt öffnet sich die Kerbe und sowohl im $\mathrm{ZrO}_{2}$ als auch im Ti werden Biegekonturen beobachtet. Der Riss wächst weiter in Richtung der benachbarten Ti-Schicht.

Im zweiten Verformungssegment des zweiten Bruchversuchs bildet sich ein Scherband (rote Markierung in Abbildung 4.28c) in der $\mathrm{ZrO}_{2}$-Schicht links neben der $\mathrm{ZrO}_{2}$-Schicht, in der ursprünglich die Kerbe liegt. Auch die nächste $\mathrm{ZrO}_{2}$-Schicht (Markierung in Abbildung 4.28b wird verformt. Außerhalb des Bildausschnittes ist während des zweiten Verformungsschrittes der Riss innerhalb der Ti-Schicht über mehr als $1 \mu \mathrm{m}$ gewachsen. An der blau markierten Stelle in Abbildung 4.28c ist zusätzlich zu erkennen, dass ein Teil des Materials aus der $\mathrm{ZrO}_{2}$-Schicht herausgelöst wird. Mit der FourierTransformation kann an dieser Stelle eine kristalline $\mathrm{ZrO}_{2}$-Schicht nachgewiesen werden, sodass diese mikrostrukturellen Veränderungen zu dem geänderten Bruchverhalten führen könnten.

In Abbildung 4.29a ist eine TEM-Übersichtsaufnahme nach dem dritten und letzten Verformungsschritt dieser Probe zu sehen. Die gesamte Risslänge beträgt ca. 4,2 um, bevor der Riss über alle Schichten hin zu der linken Si-Säule abgelenkt wird. Im dritten Segment wird zusätzlich das Scherband komplett ausgebildet und die $\mathrm{ZrO}_{2}$-Schicht an dieser Stelle durchtrennt. Nach dem Scherband verläuft der Riss in der Ti-Schicht. Der rot markierte Bereich in Abbildung 4.29a ist in höherer Vergrößerung in Abbildung $4.29 \mathrm{c} z u$ sehen. Anhand dieser Aufnahme ist eindeutig zu erkennen, dass der Riss wie auch bei den Ti100/ZrO 2100 -Proben in der Mitte einer Ti-Schicht verläuft.

In Abbildungen 4.29d und 4.29e sind Ti-Elementkarten (vgl. Abschnitt 3.1.2) an den beiden außerordentlichen Positionen des Scherbandes und der Verformung im $\mathrm{ZrO}_{2}$ gezeigt. In Abbildung 4.29d ist das herausgelöste Material dunkel dargestellt, was bedeutet, dass dieses Stück nicht aus Ti besteht. Vor und nach der Stelle mit dem herausgelösten Material liegt die Rissoberfläche innerhalb des hell dargestellten Bereichs und damit eindeutig innerhalb der Ti-Schicht. Auf der gegenüberliegenden Rissoberfläche zeigt sich auch, dass sich die Rissoberfläche in der Ti-Schicht befindet. Die Beobachtungen eines hellen Saums im Bereich des herausgelösten Materials, welches als $\mathrm{ZrO}_{2}$ identifiziert wird, ist als Artefakt der Messung zu interpretieren. Die einzelnen EFTEM- 


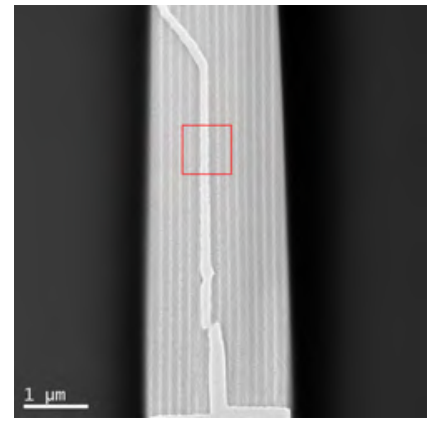

(a) nach 3. Verformung

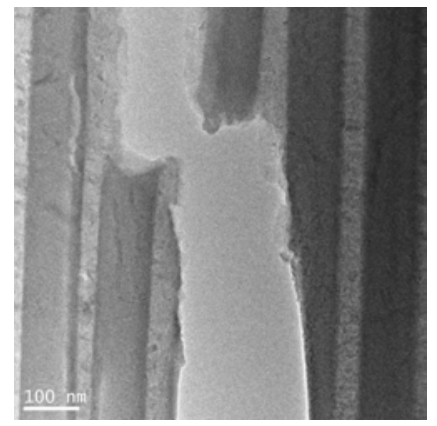

(b) Scherband

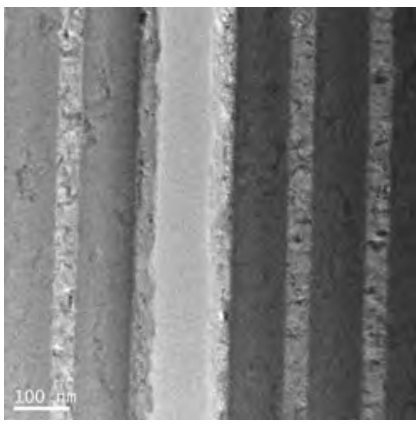

(c) Markierung aus $4.29 \mathrm{a}$

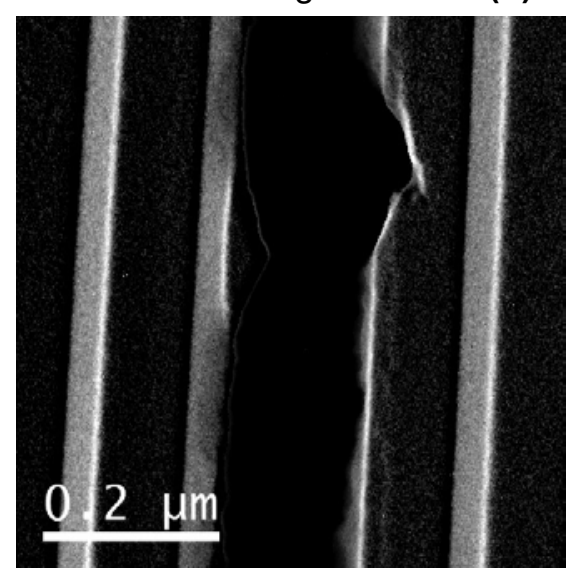

(d) Ti-Elementkarte Verformung $\mathrm{ZrO}_{2}$

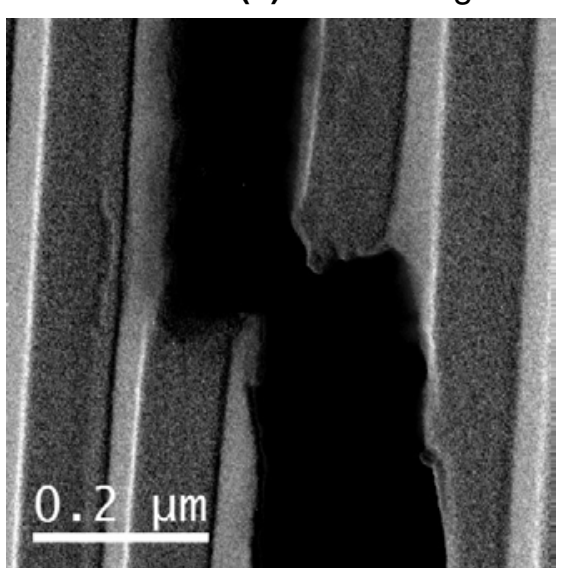

(e) Ti-Elementkarte Scherband

Abbildung 4.29: Bruchversuch Ti50/ZrO 100 entlang der Grenzflächen Probe 5

Aufnahmen dieser Stelle zeigen keine Anzeichen, die auf Ti in diesem Bereich zurück schließen lassen.

In der zweiten Elementkarte im Bereich des Scherbandes in Abbildung 4.29e ist zu beobachten, dass der Riss sowohl vor als auch nach dem Scherband in jeweils einer Ti-Schicht liegt. Jedoch liegt der Riss hier nicht in der Mitte der Ti-Schicht.

Alle weiteren Details bezüglich der Bruchversuche an Proben dieser Schichtdicken sind der Masterarbeit [Mec15] zu entnehmen.

\section{Zusammenfassung $\mathrm{Ti} 50 / \mathrm{ZrO}_{2} 100$}

Für das Ti50/ZrO 100 -Schichtdickenverhältnis bleibt zusammenfassend festzuhalten, dass auch hier bevorzugt die Rissbildung an der Kerbe stattfindet, wobei im Vergleich zu den Proben mit größerer Ti-Schichtdicke mehr Abweichungen von diesem Verhalten auftreten. Auch hier ist die Rissbildung unabhängig von dem Material, in dem die Kerbe endet. Wird der Riss in einer $\mathrm{ZrO}_{2}$-Schicht gebildet, bewegt er sich wie bei den Ti100/ZrO 100 -Proben hin zur nächsten Ti-Schicht. Risse, die in einer Ti-Schicht gebildet werden, breiten sich kontrollierbar in dieser aus. In zwei von sechs Bruchversuchen kann die Scherbandbildung in den $\mathrm{ZrO}_{2}$-Schichten beobachtet werden. 


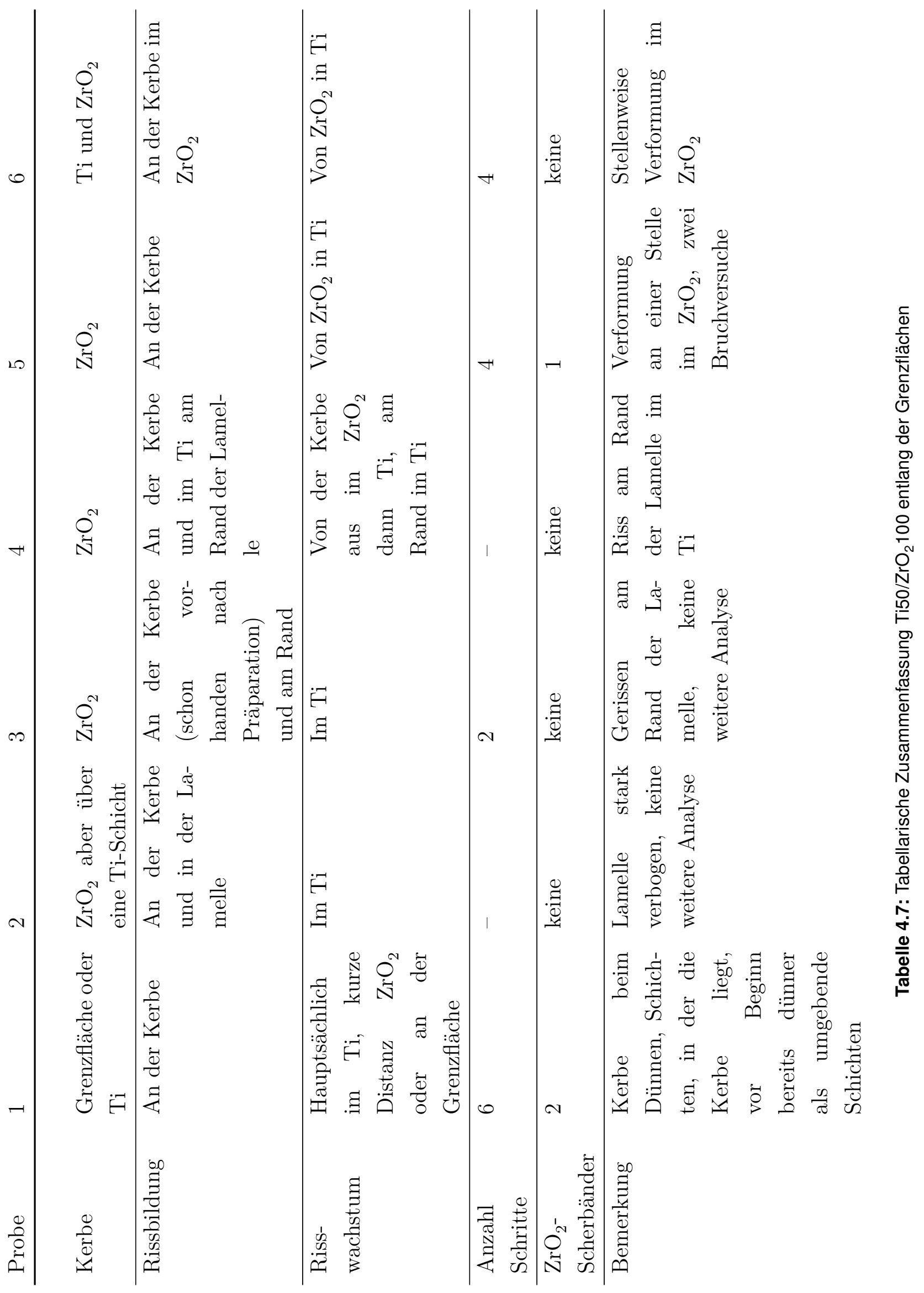




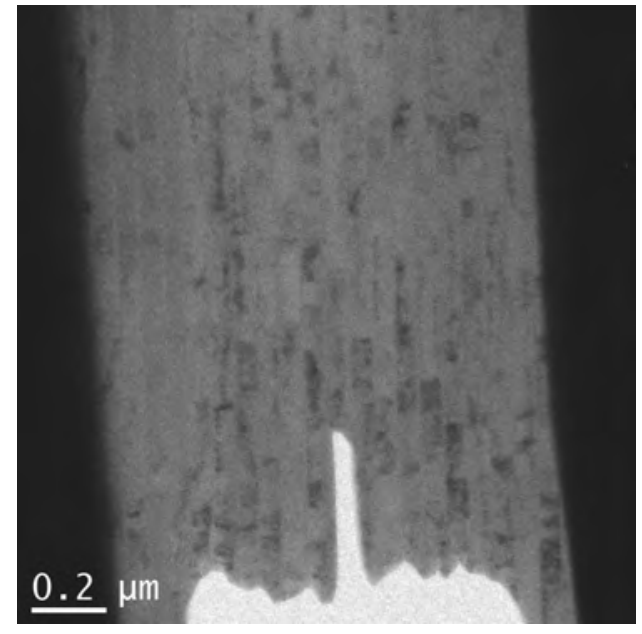

(a) BF vorher

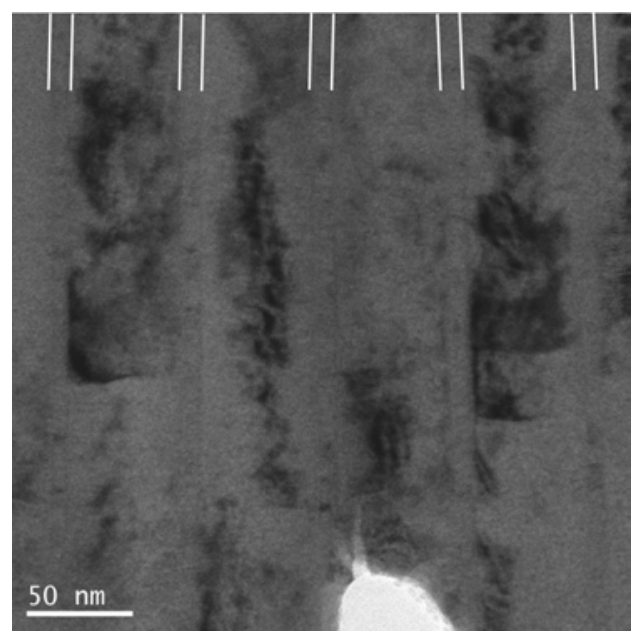

(c) Riss nach 1. Schritt

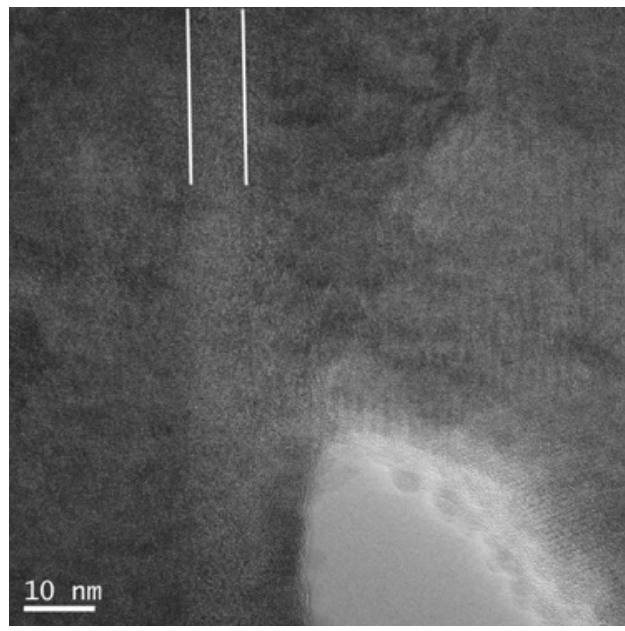

(b) BF-Kerbe

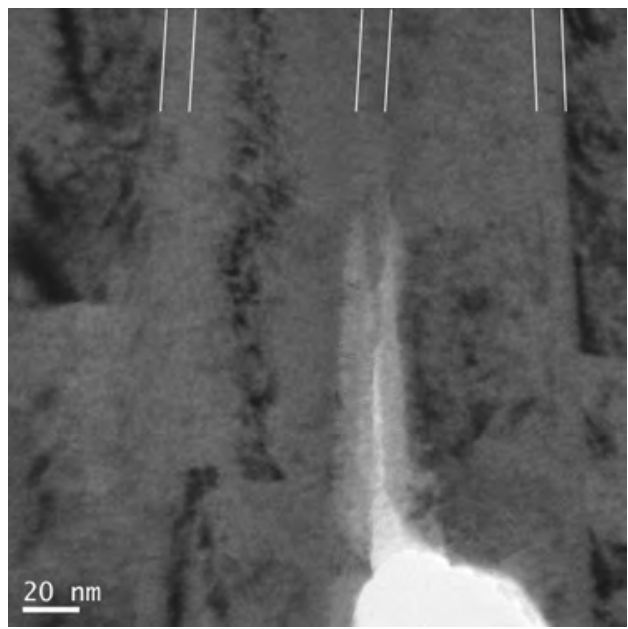

(d) Riss nach 2. Schritt

Abbildung 4.30: Bruchversuch Ti10/ZrO 100 entlang der Grenzflächen Probe 1

\subsection{3 $\mathrm{Ti} 10 / \mathrm{ZrO}_{2} 100$}

An diesem Schichtdickenverhältnis wurden drei Bruchversuche im Rahmen dieser Dissertation durchgeführt und hier dargestellt. Durch die Masterarbeit Mec15] konnten hier zusätzliche Versuche realisiert werden, sodass insgesamt sieben Bruchversuche realisiert wurden. Die Ergebnisse der zusätzlichen Versuche der Masterarbeit werden in der Zusammenfassung am Ende des Abschnittes mit einbezogen.

\section{$\mathrm{Ti10} / \mathrm{ZrO}_{2} 100-$ Probe 1}

In Abbildung 4.30a ist eine Hellfeldübersichtsaufnahme der Probe zu sehen. Die TiSchichten können bei dieser geringen Schichtdicke von ca. $10 \mathrm{~nm}$ bei geringen VergröBerungen nur noch schlecht erkannt werden. Die Positionen der Ti-Schichten sind in den Abbildungen markiert. Zur Bestimmung der Position der Kerbe ist eine höher 


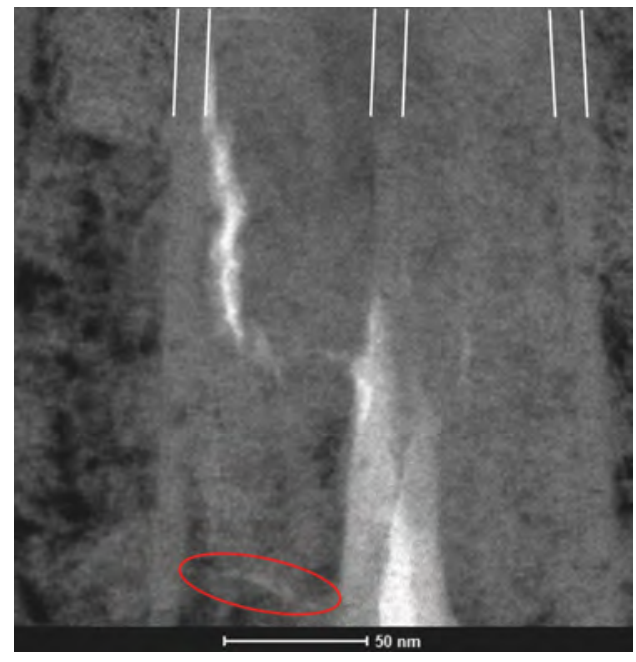

(a) Bild 460

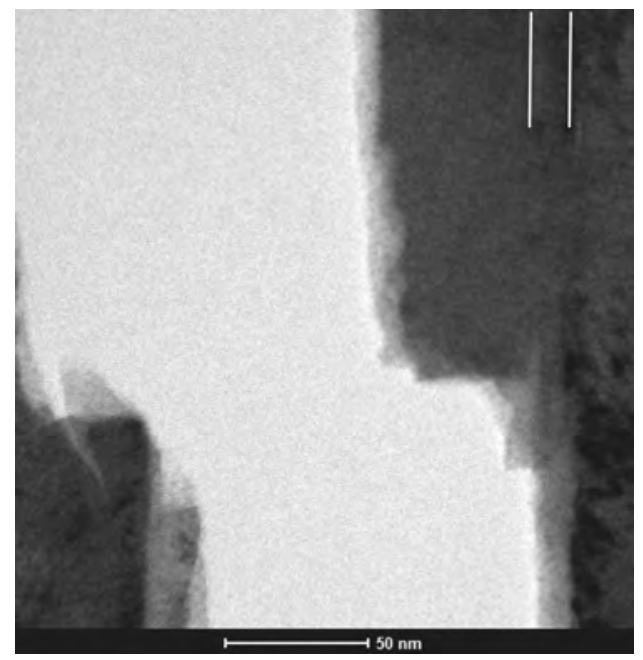

(b) Bild 462

Abbildung 4.31: Bruchversuch Ti10/ZrO 100 entlang der Grenzflächen Probe 1 Riss: (a) letzte Momentaufnahme vor dem katastrophalen Versagen; (b) erste Aufnahme nach dem Versagen

vergrößerte Aufnahme in Abbildung 4.30b dargestellt. Anhand dieser Aufnahme ist zu erkennen, dass die Kerbe und deren Spitze komplett in einer der $\mathrm{ZrO}_{2}$-Schichten liegen, wobei auf der linken Seite die nächste Ti-Schicht nur wenige nm entfernt ist. In den $\mathrm{ZrO}_{2}$-Schichten sind im Vergleich zu Proben mit dickeren Ti-Schichten mehr Biegekonturen zu beobachten und damit muss davon ausgegangen werden, dass ein größerer Teil des $\mathrm{ZrO}_{2}$ kristallin ist.

Im ersten der drei Verformungssegmente dieser Probe bildet sich ein kleiner Riss direkt an der Spitze der Kerbe (vgl. Markierung in Abbildung 4.30c). Bevor dieser Riss gebildet wird, kann die Bewegung von Biegekonturen beobachtet werden. In den TiSchichten können aufgrund der geringen Schichtdicke die Biegekonturen nicht eindeutig ausgemacht und deren Bewegung analysiert werden.

Während der zweiten Verformung wächst der gebildete Riss weiter in Richtung der auf der linken Seite benachbarten Ti-Schicht. Das Wachstum des Risses ist im zweiten Schritt begleitet von Biegekonturen in der $\mathrm{ZrO}_{2}$-Schicht. Trotz einer höher gewählten Vergrößerung der Aufnahmen in dieser Bildserie, können Biegekonturen in den Ti-Schichten nicht beobachtet werden. Der Riss am Ende des zweiten Schrittes ist in Abbildung 4.30d gezeigt. In den Einzelaufnahmen der Bildserien ist zu erkennen, dass sich der Riss in der Nähe bzw. in einer Ti-Schicht bewegt. Im Kontrast zu den bisher gezeigten Rissen liegt dieser Riss nicht in einer Ebene parallel zum Elektronenstrahl. Die Aufnahmen lassen darauf zurück schließen, dass der Riss schräg in Richtung des Elektronenstrahls verläuft.

Im dritten Schritt der Verformung dieser Probe bildet sich ein Scherband im Bildausschnitt der Bildserie, wodurch die Probe letztlich katastrophal versagt. In Abbildung $4.31 \mathrm{a}$ ist die letzte Aufnahme vor dem unkontrollierten Bruch zu sehen. Der Riss liegt 


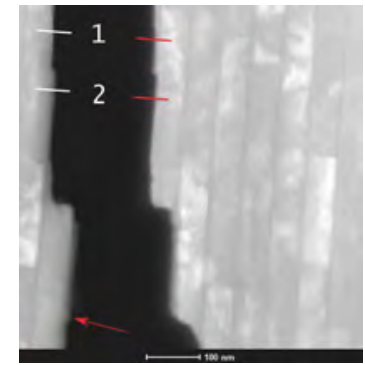

(a) HAADF Riss

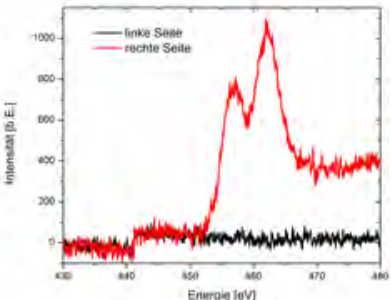

(b) EELS Pos. 1

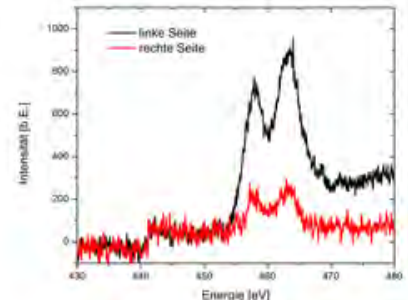

(c) EELS Pos. 2

Abbildung 4.32: Bruchversuch Ti10/ZrO 100 entlang der Grenzflächen Probe 1 EELS, Intensitätsanstieg bei etwa $440 \mathrm{eV}$ resultiert aus einem Aufnahmeartefakt der CCD-Kamera und enthält keine relevante Information

vor Beginn der letzten Verformung innerhalb der Ti-Schicht in der Mitte des Bildausschnittes. Im Verlauf der Verformung wächst der Riss voran und in der auf der linken Seite benachbarten $\mathrm{ZrO}_{2}$-Schicht bildet sich ein Riss in direkter Nähe zur Grenzfläche zur Ti-Schicht ganz links im Bildausschnitt. Zusätzlich ist ein Riss nahezu senkrecht zu den Grenzflächen in einer $\mathrm{ZrO}_{2}$-Schicht zu erkennen (Markierung in Abbildung 4.31a). In dem übernächsten Bild dieser Bildserie hat sich der Riss unkontrolliert durch die gesamte Lamelle ausgebildet (vgl. Abbildung 4.31b). Während des gesamten Verformungsschrittes kann wie zuvor nur die Bewegung der Biegekonturen in den $\mathrm{ZrO}_{2}{ }^{-}$ Schichten eindeutig beobachtet werden.

Abbildung 4.32a zeigt eine HAADF-Übersichtsaufnahme des Risses am Ende des Bruchversuchs. Um den Verlauf des Risses eindeutiger bestimmen zu können, werden an zwei Stellen EELS-Linienaufnahmen realisiert. Entlang der dargestellten Linien in Abbildung 4.32a werden mehrere EELS-Einzelspektren im Energiefenster der Ti-LKante aufgenommen. Die EELS-Spektren an den jeweiligen Rissoberflächen können so Aufschluss darüber geben, ob der Riss an dieser Stelle in der Ti-Schicht verläuft.

In Abbildungen 4.32b und 4.32c sind die oberflächennahen EELS-Spektren der beiden markierten Positionen gezeigt. Der Intensitätsanstieg bei der Energie von etwa $441 \mathrm{eV}$ ist als Aufnahmeartefakt der CCD-Kamera des Spektrometers zu interpretieren und wird nicht weiter diskutiert. Vielmehr ist im Vergleich der Spektren zu erkennen, dass bei Position 2 an beiden Rissoberflächen ein Ti-Signal gemessen wird und bei Position 1 nur an der rechten Oberfläche des Risses. Dieses Ergebnis lässt darauf schließen, dass der Riss an Position 2 in der Ti-Schicht verläuft und an Position 1 an der Grenzfläche.

\section{Ti10/ZrO 2 100-Probe 2}

Bei dieser Probe liegt die Spitze der Kerbe komplett in einer einzelnen $\mathrm{ZrO}_{2}$-Schicht, ohne eine Ti-Schicht zu berühren. Der entsprechende Bruchversuch ist in vier Abschnitte unterteilt. In den ersten drei Abschnitten, die als Dunkelfeldbildserien aufgenommen 


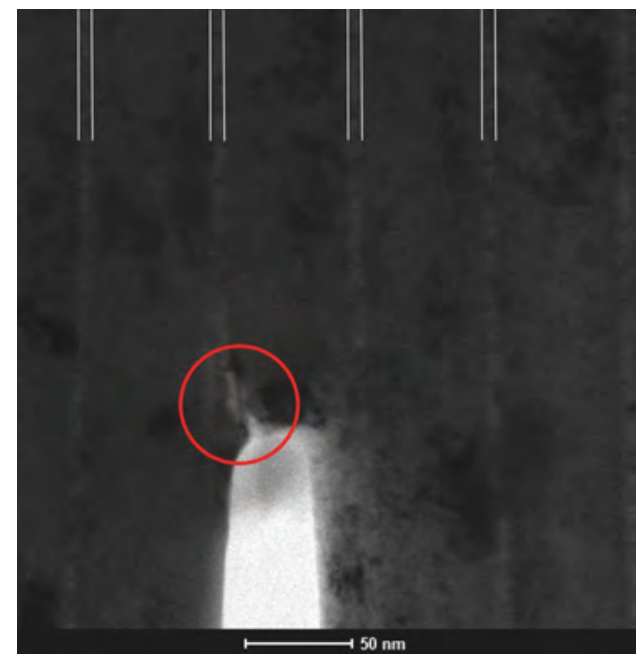

(a) Bild 404

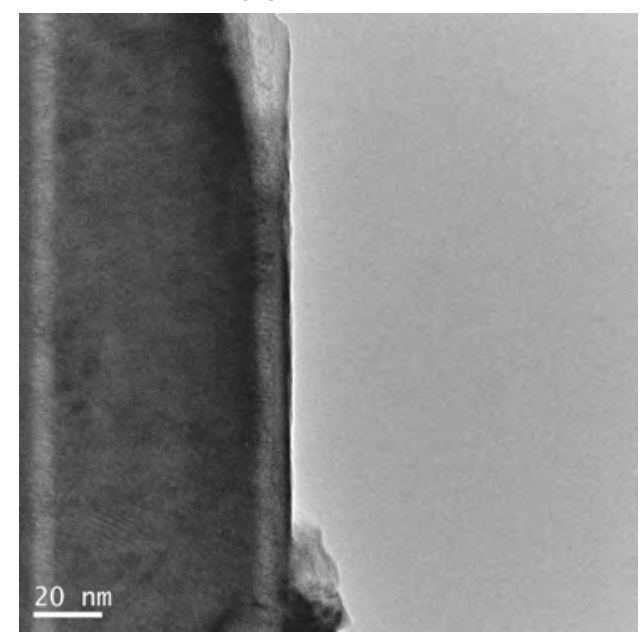

(c) linke Seite des Risses

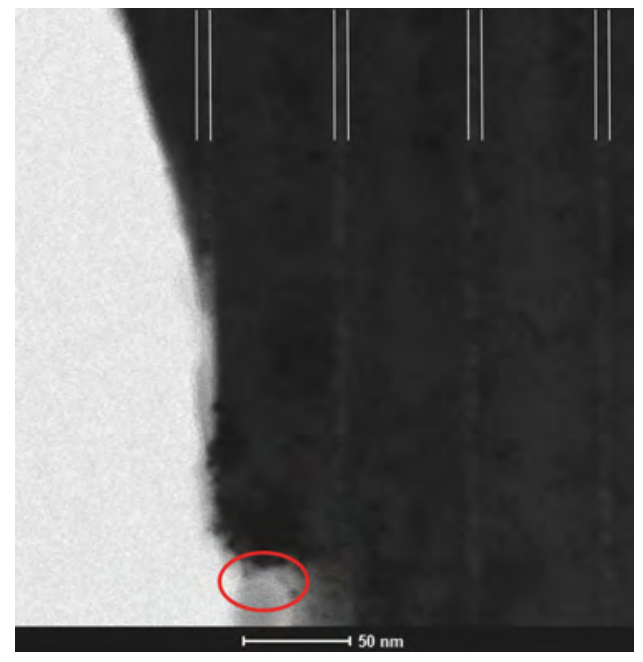

(b) Bild 406

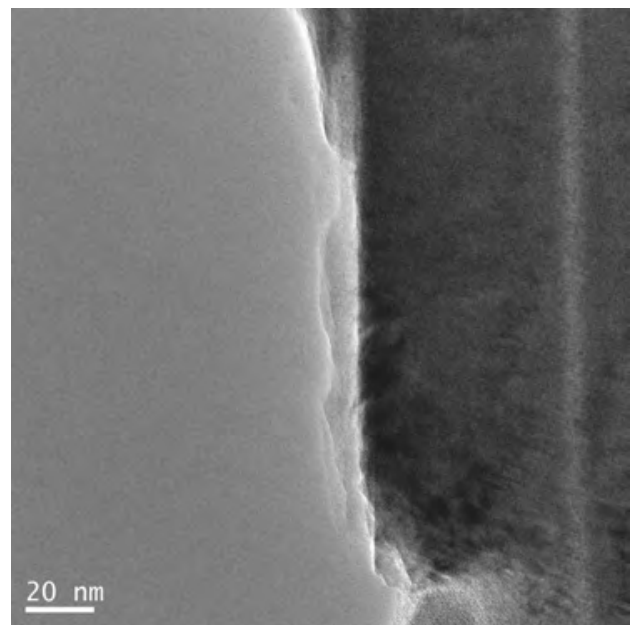

(d) rechte Seite des Risses

Abbildung 4.33: Bruchversuch Ti10/ZrO 100 entlang der Grenzflächen Probe 2: (a) letzte Aufnahme vor dem Versagen; (b) erste Aufnahme nach dem Versagen

sind, ist nur eine geringe Vergrößerung der Öffnungsweite der Kerbe zu beobachten, die jedoch deutlich geringer ist zu den Veränderungen in Proben mit größeren TiSchichtdicken. Zusätzlich kann innerhalb der $\mathrm{ZrO}_{2}$-Schichten ebenfalls die Bewegung einiger Biegekonturen beobachtet werden, was darauf schließen lässt, dass die $\mathrm{ZrO}_{2^{-}}$ Schichten kristallisiert sind. In den Ti-Schichten können aufgrund der Schichtdicke und der Vergrößerung nur sehr wenige eindeutige Kontrastveränderungen entweder durch Biegekonturen oder durch Versetzungsaktivität beobachtet werden.

Die Abbildungen 4.33a und 4.33b zeigen zwei Momentaufnahmen aus der Bildserie des letzten Verformungsschrittes. In beiden Abbildungen sind am oberen Rand die TiSchichten markiert. Es ist zu erkennen, dass sich der Riss an der Spitze der Kerbe gebildet hat und in Richtung der am nächsten liegenden Ti-Schicht abgelenkt wird (rote Markierung in Abbildung 4.33a). Bevor jedoch der Riss die Ti-Schicht erreicht, 
versagt die Probe in einer katastrophalen Art und Weise. In Abbildung 4.33b ist eine Momentaufnahme direkt nach dem Bruch der Probe gezeigt. Diese Aufnahme ist ca. 0,2 s nach dem Bild in Abbildung 4.33a aufgenommen. Die Markierung in 4.33b zeigt die ursprüngliche Spitze der Kerbe, sodass eindeutig erkannt werden kann, dass der zuvor gebildete Riss unkontrolliert wächst und zum Bruch der gesamten Probe führt. Das unkontrollierte Risswachstum geht über alle Grenzflächen hinweg schräg durch die gesamte Lamelle hin zu einer der beiden Si-Säulen.

In höher vergrößerten Aufnahmen (Abbildungen 4.33c und 4.33d) wird der Riss genauer analysiert. In der Nähe der Kerbe ist zu beobachten, dass der Riss über eine Distanz von ca. $100 \mathrm{~nm}$ parallel zu einer Grenzfläche verläuft. Mittels Fourier-Transformationen an den jeweiligen Rissoberflächen können kristalline Strukturen auf beiden Seiten des Risses nachgewiesen werden. Damit ist anzunehmen, dass der Riss in diesem Bereich in einer Ti-Schicht verläuft. Jedoch ist auch eindeutig ein Unterschied zu den bisherigen Rissverläufen in einer Ti-Schicht zu erkennen. Aufgrund des hellen Kontrastes der Ti-Schicht an der Rissoberfläche in Abbildung 4.33d kann zusätzlich angenommen werden, dass hier weniger Ti vorliegt im Vergleich zur Ti-Schicht in Abbildung 4.33c

\section{$\mathrm{Ti10} / \mathrm{ZrO}_{2} 100-$ Probe 3}

Die letzte im Rahmen der Dissertation untersuchte Probe dieses Schichtdickenverhältnisses ist in Abbildung 4.34 dargestellt. Wie schon in der Hellfeldaufnahme (Abbildung 4.34a zu erkennen ist, ist die Kerbe im FIB nicht komplett durchgeschnitten worden. Gerade an der Spitze der Kerbe ist noch ein kleiner Bereich des Materials stehen geblieben. In der dynamischen Dunkelfeldaufnahme in Abbildung 4.34b ist dieser dünnere Bereich dunkler dargestellt. Die Position der Spitze der Kerbe ist in diesem Fall nicht eindeutig zu bestimmen. Die Stelle, an der die Lamelle komplett durchgeschnitten ist, liegt sowohl in einer Ti- als auch in einer $\mathrm{ZrO}_{2}$-Schicht. Die Spitze des ausgedünnten, aber nicht durchtrennten Bereichs liegt hingegen ausschließlich in einer $\mathrm{ZrO}_{2}$-Schicht. Dieser Bruchversuch ist insgesamt in acht Verformungssegmente unterteilt. In den ersten drei Verformungsschritten kann eine Vergrößerung der Öffnungsweite der ursprünglichen Kerbe beobachtet werden. In allen Bildserien ist eine Bewegung der Biegekonturen in den $\mathrm{ZrO}_{2}$-Schichten offensichtlich. Am Ende der Bildserie des dritten Schrittes, die wie auch die ersten beiden Serien in Dunkelfeldabbildungsbedingungen aufgenommen werden, verdunkelt sich der dünnere Bereich an der Spitze der Kerbe. In diesem Bereich hat sich ein Riss gebildet. In Abbildung 4.35a ist eine Hellfeldaufnahme am Ende des dritten Schrittes gezeigt, die bestätigt, dass sich ein kleiner Riss in dem dünneren Bereich der Spitze gebildet hat (vgl. Markierung in Abbildung 4.35a).

Im vierten und fünften Schritt wächst der Riss ausgehend von dem kleinen Riss in der $\mathrm{ZrO}_{2}$-Schicht. Die Ausbreitung liegt in Richtung der auf der rechten Seite benachbar- 


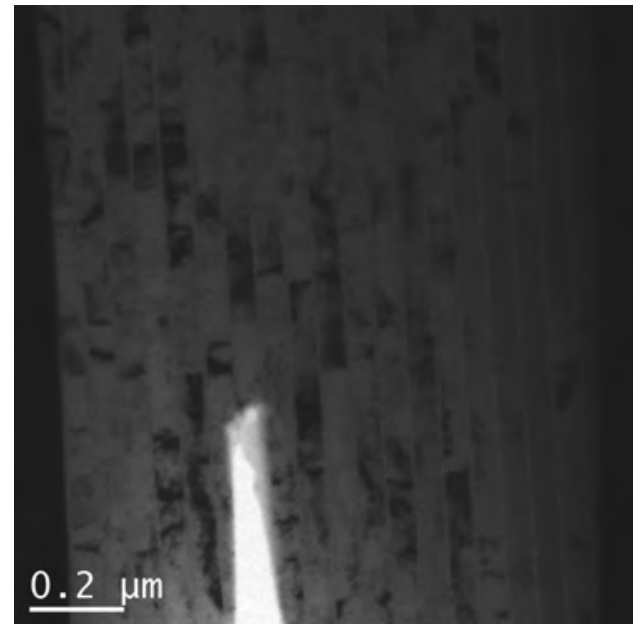

(a) BF vorher

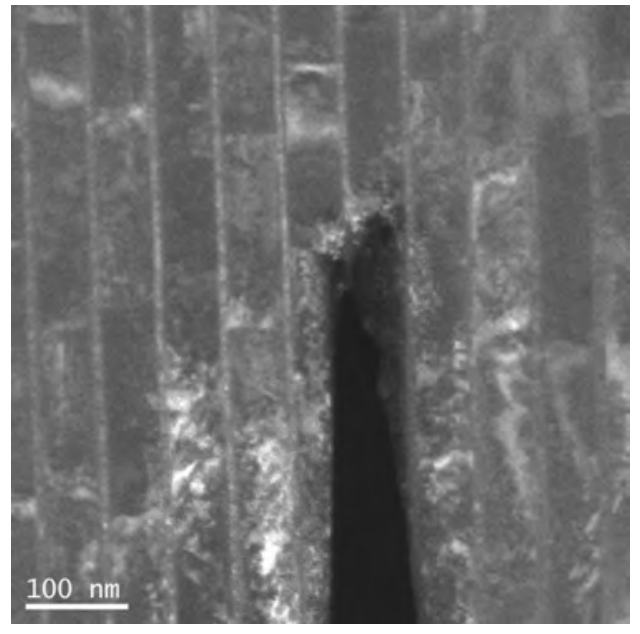

(b) dyn. DF Kerbe

Abbildung 4.34: Bruchversuch $\mathrm{Ti10} / \mathrm{ZrO}_{2} 100$ entlang der Grenzflächen Probe 3

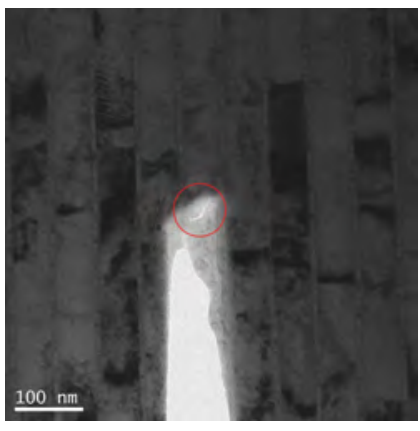

(a) nach 3. Schritt

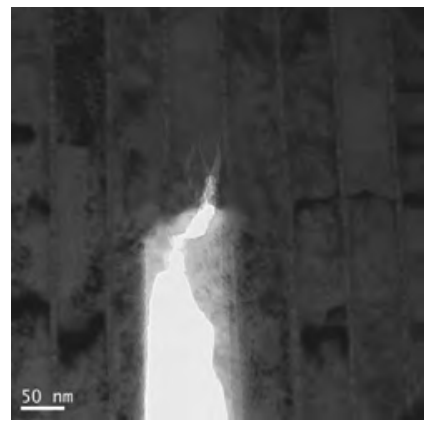

(b) nach 5. Schritt

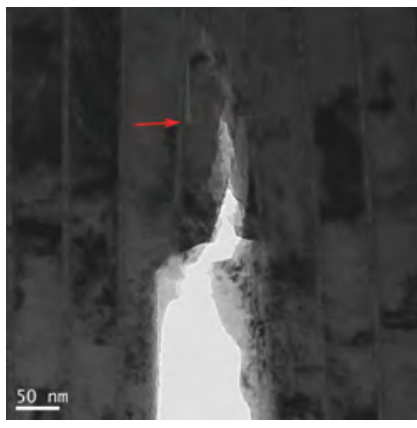

(c) nach 7. Schritt

Abbildung 4.35: Bruchversuch $\mathrm{Ti} 10 / \mathrm{ZrO}_{2} 100$ entlang der Grenzflächen Probe 3

ten Ti-Schicht. Auch der dünnere Bereich an der Spitze der Kerbe reißt in den beiden Bildserien komplett durch. An der Spitze des Risses bilden sich mehrere Rissspitzen aus, wie in Abbildung 4.35b zu sehen.

Im sechsten und siebten Schritt der Verformung wächst der Riss deutlich in Richtung der Grenzfläche zur benachbarten Ti-Schicht. Wie auch schon bei den vorherigen Proben, lässt die Form der Rissoberflächen darauf schließen, dass der Riss schräg zur Lamelle liegt (in Richtung der Lamellendicke siehe Skizze 4.36e). Am Ende des siebten Schrittes bildet sich wiederum ein Riss in der Nähe der Grenzfläche zu einer neuen Ti-Schicht (Pfeil in Abbildung 4.35c).

Im achten und letzten Verformungsschritt zeigt sich wie im siebten Schritt ein Scherband über eine weitere $\mathrm{ZrO}_{2}$-Schicht und entlang der beiden gebildeten Scherbänder versagt die Probe letztlich katastrophal. Wie schon in den anderen beiden geschilderten Fällen wächst der Riss in einer unkontrollierten Weise und die Probe ist innerhalb zweier Einzelbilder der Bildserie komplett gerissen.

In Abbildung 4.36 sind zwei zusammengesetzte Übersichtsaufnahmen des Risses am En- 


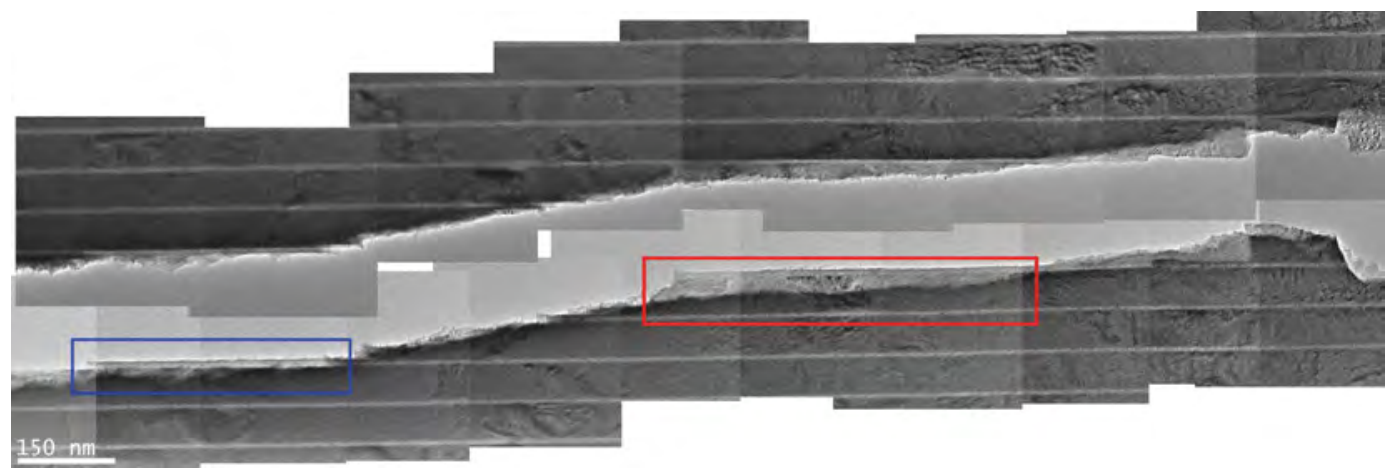

(a) Übersicht Riss

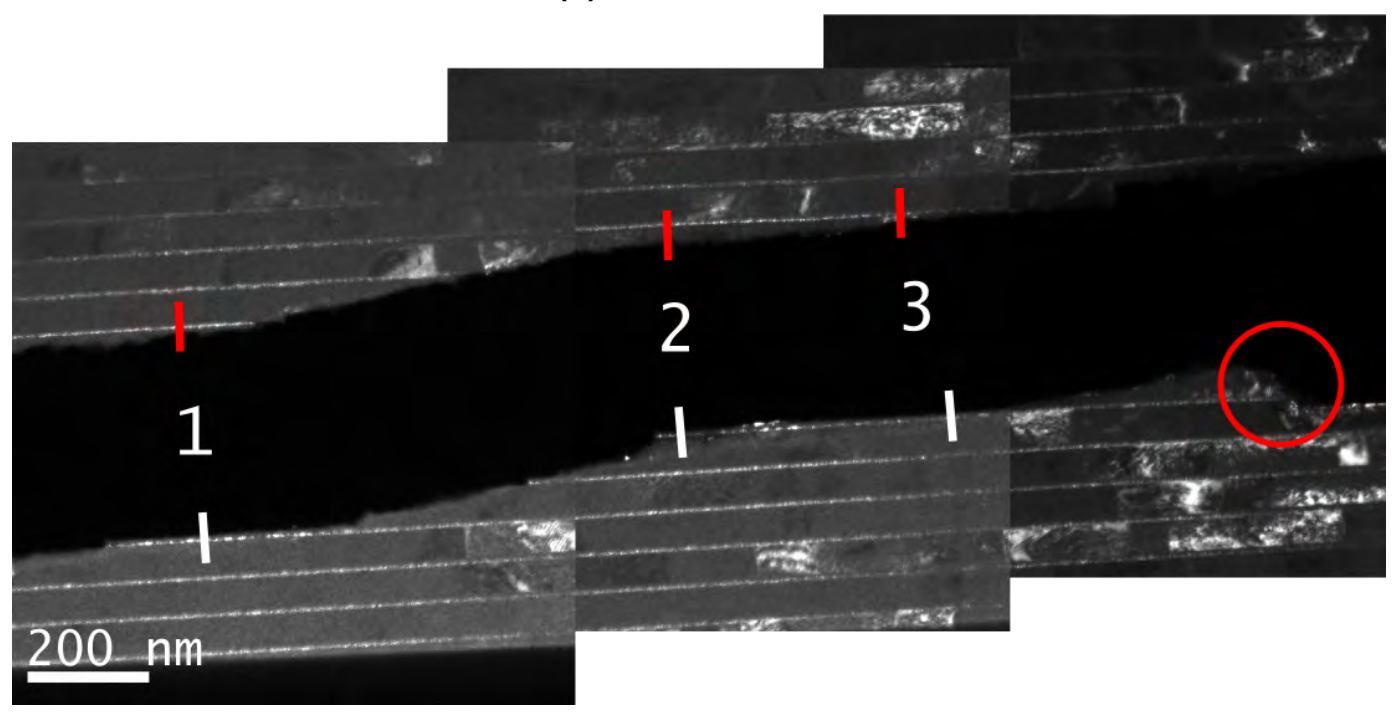

(b) DF Übersicht Riss mit Positionen für EELS

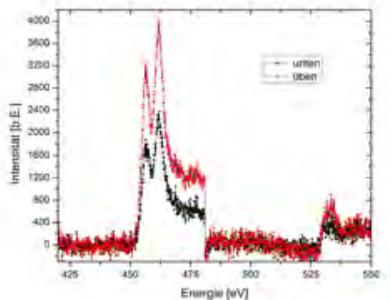

(c) EELS Position 1

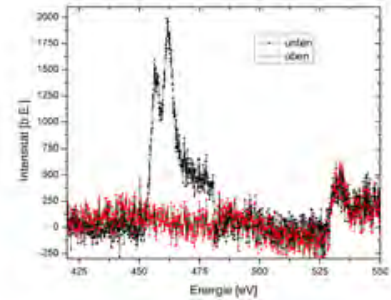

(d) EELS Position 2

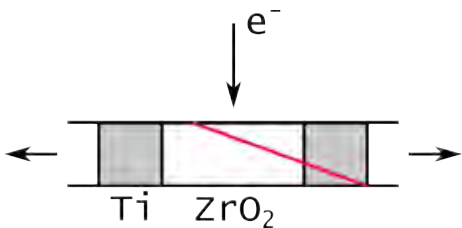

(e) Rissverlauf

Abbildung 4.36: Bruchversuch $\mathrm{Ti} 10 / \mathrm{ZrO}_{2} 100$ entlang der Grenzflächen Probe 3 Übersicht Riss

de des Bruchversuchs gezeigt. Die ursprüngliche Spitze der Kerbe liegt jeweils rechts (Markierung in Abbildung 4.36b). Der Rissverlauf ist vergleichbar zu den bisher beobachteten Ergebnissen. Der Riss verläuft stellenweise schräg entlang der Lamellendicke (rote Markierung in Abbildung4.36a). Es können jedoch auch Stellen beobachtet werden, an denen zu vermuten ist, dass der Riss entlang der Grenzfläche zwischen den beiden Materialien verläuft (zum Beispiel blaue Markierung in Abbildung 4.36a). Anhand von EELS-Analysen an den drei gekennzeichneten Positionen in Abbildung 4.36b wird der genaue Rissverlauf bestimmt. Zusätzliche Informationen bezüglich des Riss- 
verlaufs können EFTEM-Elementkarten liefern (hier nicht gezeigt).

Im Bereich der blauen Markierung in Abbildung 4.36a liefern sowohl die EFTEM-TiElementkarten als auch die gezeigten EELS-Spektren an der jeweiligen Rissoberfläche (Position 1) das gleiche Ergebnis - an beiden Rissoberflächen kann ein Ti-Signal nachgewiesen werden. In der Übersicht in Abbildung 4.36a ist jedoch zu erkennen, dass es im Bereich der blauen Markierung Stellen gibt, an denen Teile des $\mathrm{ZrO}_{2}$ verformt bzw. gerissen und diese Teile der $\mathrm{ZrO}_{2}$-Schicht an der gegenüberliegenden Rissoberfläche zu finden sind.

Im Bereich der roten Markierung kann an der unteren Rissoberfläche eindeutig ein Ti-Signal in den EELS-Daten gefunden werden, an der korrespondierenden, oberen Rissoberfläche jedoch keines. Diese Beobachtung kann anhand der Abbildung 4.36a nachvollzogen werden. Innerhalb der Markierung ist der Rissverlauf, in Richtung der Lamellendicke betrachtet, nicht parallel zu den Grenzflächen. Vielmehr kommt es hier zu einem Abscheren des Materials über die Grenzflächen hinweg (vgl. Skizze in Abbildung 4.36e. Im Bereich der roten Markierung ist die Oberfläche des Risses gleichzusetzen mit der Grenzfläche zwischen einer Ti- und einer $\mathrm{ZrO}_{2}$-Schicht.

An Position 3 in Abbildung 4.36b kann auf beiden Oberflächen des Risses ein Ti-Signal nachgewiesen werden. Im Gegensatz dazu ist im Bereich der Spitze der ursprünglichen Kerbe in den Ti-Elementkarten kein Ti-Signal an der Rissoberfläche zu beobachten (Abbildungen hier nicht gezeigt).

Den gesamten Rissverlauf betrachtet, bleibt das Ergebnis festzuhalten, dass sich der Riss sowohl im $\mathrm{ZrO}_{2}$ als auch im Ti oder stellenweise an der Grenzfläche ausgebreitet hat.

\section{Zusammenfassung $\mathrm{Ti10} / \mathrm{ZrO}_{2} 100$}

Im Vergleich zu den beiden Proben mit dickeren Ti-Schichtdicken zeigen die Lamellen mit der geringsten untersuchten Ti-Schichtdicke ein verändertes Bruchverhalten. Ausschließlich bei den dünnen Ti-Schichten kann beobachtet werden, dass der Riss sich nicht nur in der Ti-Schicht ausbreitet, sondern auch entlang der Grenzfläche zwischen der Ti- und der $\mathrm{ZrO}_{2}$-Schicht. Außerdem verändert sich das Bruchverhalten dahingehend, dass sich der Riss nicht mehr kontrollierbar ausbreitet. Vielmehr versagen alle Lamellen katastrophal. Die Rissbildung sowie die Beobachtung von Scherbändern (in drei von sieben Lamellen) ist jedoch vergleichbar zu den Bruchversuchen größerer TiSchichtdicken. 


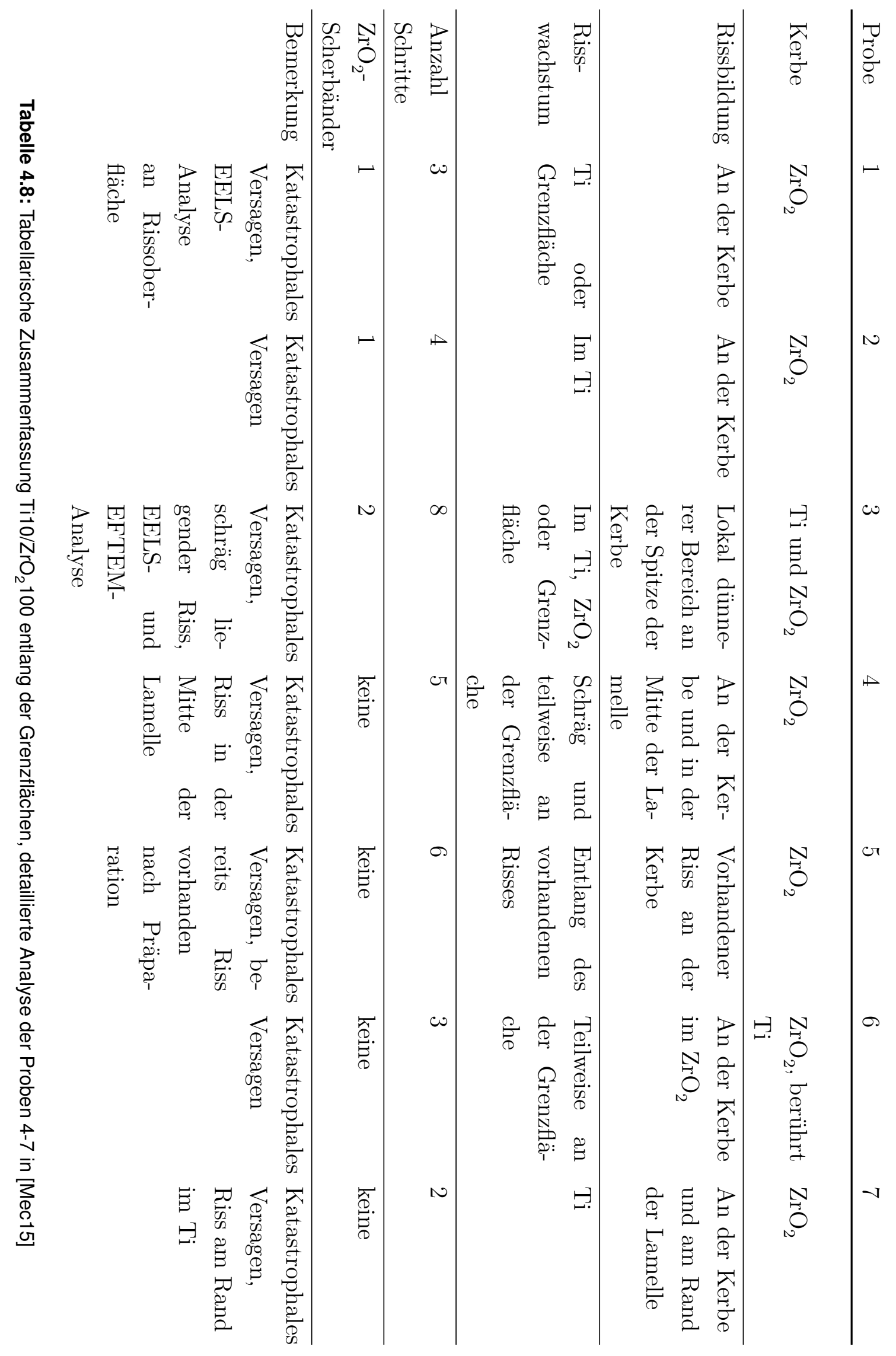




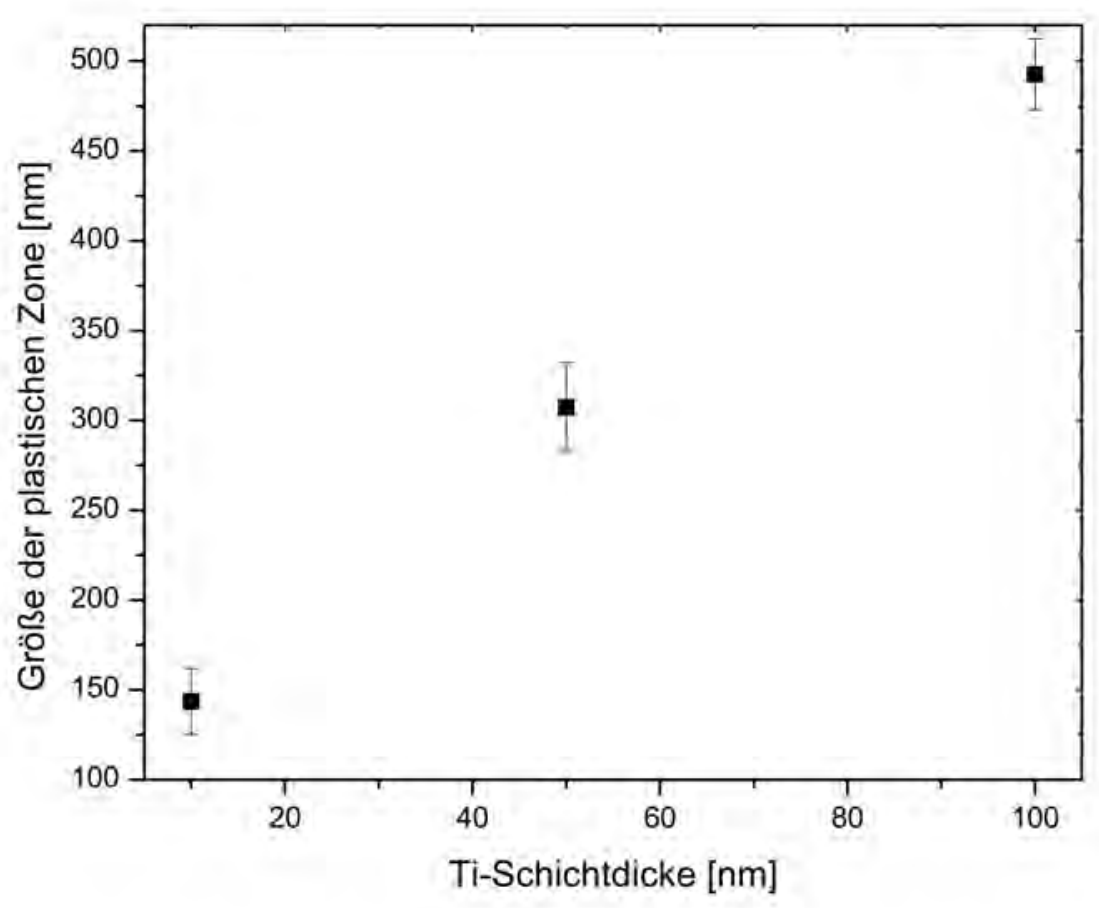

Abbildung 4.37: Größe der plastischen Zone $r_{p}$ entlang der Grenzflächen

\subsubsection{Plastische Zone}

Für alle Bildserien wird die Größe der plastischen Zone anhand der schnell wechselnden Kontraste in den Ti-Schichten bestimmt. In Abschnitt 3.4.2 ist die Vorgehensweise exemplarisch für die Ti-Einzelschichten beschrieben und demonstriert. Im Fall der Multilagen mit den Ti-Schichtdicken von $50 \mathrm{~nm}$ und $100 \mathrm{~nm}$ wird die plastische Zone wie für die Ti-Einzelschichten anhand der Versetzungsaktivität in den Ti-Schichten bestimmt. Für die Proben mit der kleinsten Schichtdicke ist diese Auswertung deutlich schwieriger, da die Kontrastwechsel in den Ti-Schichten bei den gewählten Vergrößerungen nicht eindeutig erkannt werden können. Hier dienen ähnliche Kontrastwechsel in den stellenweise kristallinen $\mathrm{ZrO}_{2}$-Schichten als Abschätzung der Größe der plastischen Zone.

In Abbildung 4.37 sind die Ergebnisse gegen die Ti-Schichtdicke in den Multilagen aufgetragen. Die dargestellten Werte verstehen sich als gewichtete Mittelwerte der ausgemessenen plastischen Zonen verschiedener Proben einer Schichtdicke. 


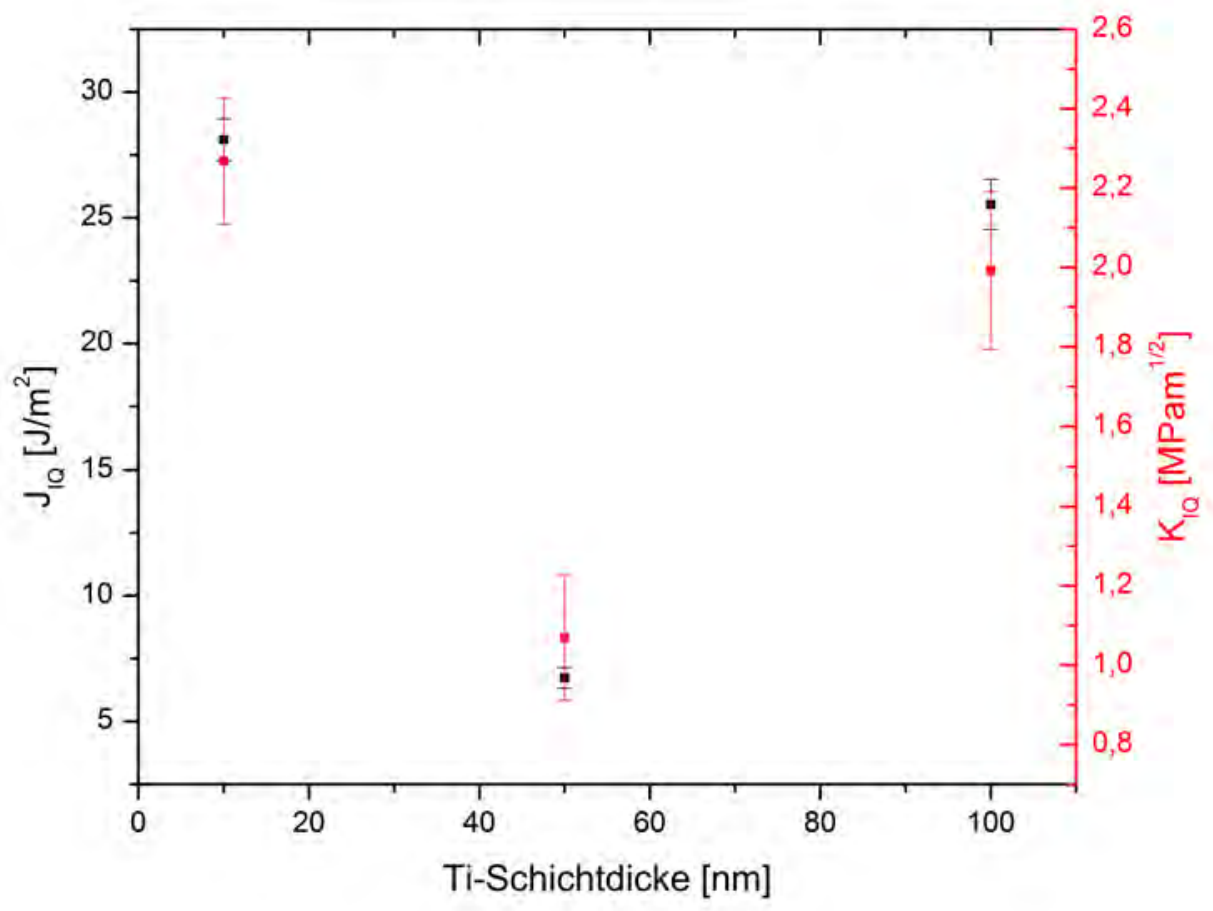

Abbildung 4.38: Energiefreisetzungsrate $J_{I Q}$ und Bruchzähigkeit $K_{I Q}$ entlang der Grenzflächen

\subsubsection{Energiefreisetzungsrate}

Für alle geeigneten Bildserien kann die Risswiderstandskurve aufgetragen und entsprechend der beschriebenen Prozedur (vgl. Abs. 3.4.1) ausgewertet werden. In Abbildung 4.38 sind die kritischen Werte der Energiefreisetzungsrate $J_{I Q}$ und die daraus entsprechend Gleichung (3.3) errechneten kritischen Bruchzähigkeiten $K_{I Q}$ dargestellt. Die aufgetragenen Werte verstehen sich auch hier als gewichtete Mittelwerte der einzelnen Bruchversuche verschiedener Ti-Schichtdicken. In Abbildung 4.39 sind exemplarisch die Risswiderstandskurven für jedes Schichtdickenverhältnis gezeigt. 


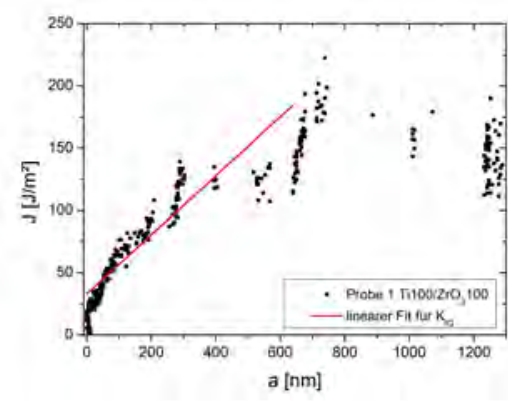

(a) $\mathrm{Ti100} / \mathrm{ZrO}_{2} 100$ Probe 1

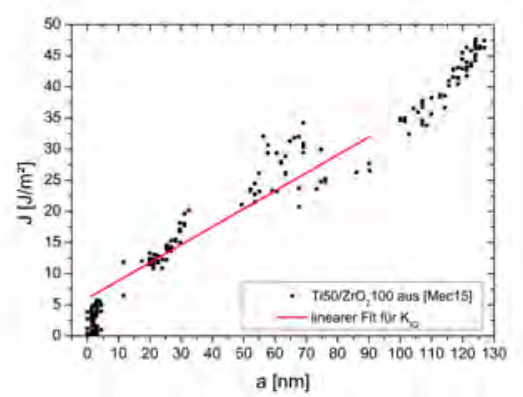

(b) $\mathrm{Ti} 50 / \mathrm{ZrO}_{2} 100$ aus $\mid \mathrm{Mec} 15$

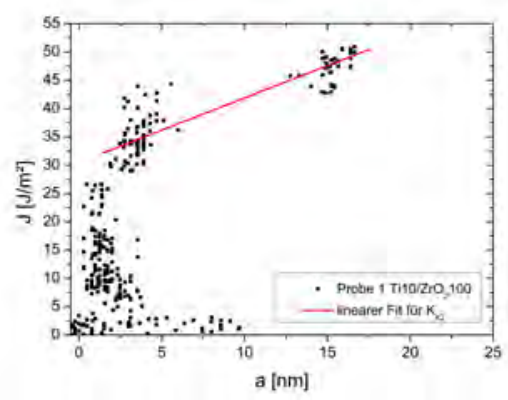

(c) $\mathrm{Ti} 10 / \mathrm{ZrO}_{2} 100$ Probe 1

Abbildung 4.39: Risswiderstandskurven Bruchversuche entlang der Grenzflächen 


\subsection{Bruchversuche senkrecht zu den Grenzflächen}

In diesem Abschnitt werden die gewonnenen Erkenntnisse der Bruchversuche mit einer Rissausbreitung senkrecht zu den $\mathrm{Ti} / \mathrm{ZrO}_{2}$-Grenzflächen dargestellt und beschrieben. Wie schon im vorherigen Abschnitt werden die Ergebnisse der einzelnen untersuchten Proben der verschiedenen Ti-Schichtdicken aufgezählt und abschließend eine Zusammenfassung der Ergebnisse der entsprechenden Ti-Schichtdicke angegeben. Zusätzlich werden zum Schluss dieses Abschnittes die quantitativen Ergebnisse der Bruchversuche senkrecht zur Grenzfläche bezüglich der Größe der plastischen Zone sowie der kritischen Bruchzähigkeit bei der Rissbildung in Abhängigkeit der Ti-Schichtdicke dargestellt.

\subsection{1 $\mathrm{Ti} 100 / \mathrm{ZrO}_{2} 100$}

Ti100/ZrO $2100-$ Probe 1

In Abbildung 4.40a ist eine Dunkelfeldübersichtsaufnahme der ersten untersuchten Probe in der Geometrie für die Rissausbreitung senkrecht zu den Grenzflächen gezeigt. Die Präparation dieser Geometrie unterscheidet sich nur geringfügig von der Geometrie der Bruchversuche entlang der Grenzflächen (vgl. Abschnitt 3.3.1). Am linken Rand der Lamelle ist ein Ti-Tropfen zu sehen, der während der Herstellung der Multilagen entstanden ist. Auf diesem Tropfen wachsen die weiteren Schichten im Verlauf der Präparation der Multilagen versetzt bzw. verkippt auf. Da dieser Tropfen am Rand der Lamelle und damit eine gewisse Distanz entfernt von der Spitze der Kerbe liegt, ist nicht zu erwarten, dass durch den Tropfen das Rissverhalten signifikant beeinflusst wird.

Die Spitze der Kerbe ist in Abbildung $4.40 \mathrm{~b} z u$ sehen. Anhand dieser Aufnahme ist zu erkennen, dass die Position der Spitze der Kerbe eindeutig in einer $\mathrm{ZrO}_{2}$-Schicht liegt, jedoch nah der Grenzfläche zu der nächsten Ti-Schicht.

Dieser Bruchversuch ist in sieben Verformungsschritte unterteilt, wobei nach dem vierten Verformungsschritt die Probe entlastet wird, da der gesamte Verfahrweg der STMSpitze im STM-TEM-Halter erreicht worden und keine weitere Verformung möglich ist. Nach der Entlastung wird der „neue“ Ausgangszustand charakterisiert und eine weitere Verformung realisiert. Alle Verformungsschritte werden für die Probe in Dunkelfeldbildserien dokumentiert.

In dem ersten Verformungsschritt können neben einer kleinen Vergrößerung der Öffnungsweite der Kerbe keine weitere Verformung oder Kontrastveränderungen beobachtet werden. In dem zweiten Schritt hingegen ist eine deutliche Vergrößerung der Öffnungsweite zu beobachten, jedoch ist noch keine Verformung in der Nähe der Spitze der Kerbe oder eine Rissbildung zu erkennen. Während dieses zweiten Schrittes kann 


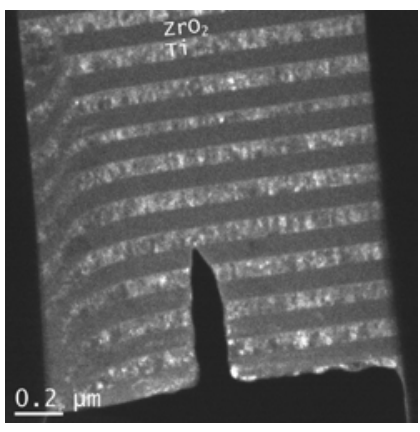

(a) DF-Übersicht

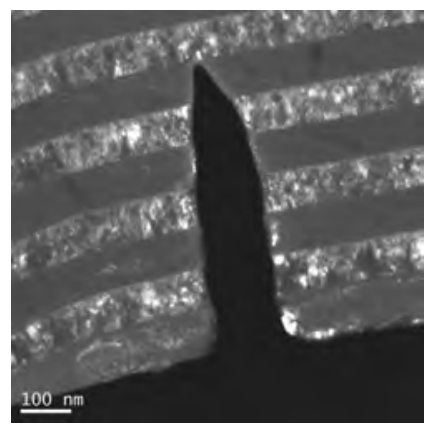

(b) DF-Kerbe

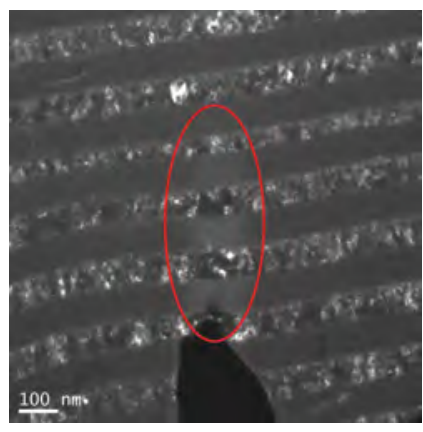

(c) DF Kerbe nach 4. Schritt

Abbildung 4.40: Bruchversuch $\mathrm{Ti100} / \mathrm{ZrO}_{2} 100$ senkrecht zu den Grenzflächen Probe 1

aber eindeutig die Aktivität von Versetzungen in den Ti-Schichten beobachtet werden. Der Bereich der Versetzungsaktivität reicht bis zu der Ti-Schicht, die 15 Einzelschichten entfernt von der Kerbe ist, was einer Distanz von mehr als $1 \mu \mathrm{m}$ entspricht. Im dritten und vierten Schritt der Verformung öffnet sich die Kerbe stetig weiter und die Spitze der Kerbe wird stumpfer (siehe Abbildung 4.40c). Zusätzlich ist zu erkennen, dass die $\mathrm{ZrO}_{2}$-Schichten innerhalb der Markierung in Abbildung 4.40c heller erscheinen, was mit einer Reduzierung der Dicke der Lamelle in Elektronenstrahlrichtung einhergeht. Weiterhin ist eine Verbiegung der Schichten in der Markierung zu beobachten. Eine eindeutige Rissbildung ausgehend von der Kerbe kann nicht festgestellt werden, jedoch ist zweifelsfrei zu erkennen, dass die Spitze der ursprünglichen Kerbe am Ende der vier Verformungsschritte in der benachbarten Ti-Schicht lokalisiert ist.

In einer weniger vergrößerten Aufnahme der gesamten Lamelle ist zu sehen, dass sich am Rand des Ti-Tropfens ein weiterer Riss gebildet hat (Markierung in Abbildung 4.41a. Dieser Riss breitet sich entlang der Grenzfläche zwischen Tropfen und den umgebenden Schichten über drei Ti-Schichten in Richtung der oberen Kante der Lamelle (unterer Rand in den Abbildungen) hin aus.

In der zweiten Verformung (nach vorherig entlasteter Probe) wird die Probe in den Verformungsschritten fünf bis sieben erneut belastet und die Rissbildung beobachtet. Abbildung 4.41a zeigt eine Hellfeldübersichtsaufnahme vor der zweiten Belastung der Probe. Außerdem ist zu erkennen, dass sowohl die Ti- als auch die $\mathrm{ZrO}_{2}$-Schicht vor der Spitze der Kerbe leicht heller erscheinen als die anderen Schichten, sodass diese Schichten in der Nähe der Kerbe dünner als die benachbarten Schichten sind. Im ersten Schritt der zweiten Verformung (insgesamt der fünfte Schritt) wird lediglich der Kontakt mit der Lamelle und eine minimale Öffnung der Kerbe erreicht. Rissbildung oder Versetzungsaktivität kann nicht beobachtet werden. Im sechsten Schritt bildet sich der Riss an der Spitze der Kerbe und breitet sich in Richtung der Grenzfläche zur benachbarten $\mathrm{ZrO}_{2}$-Schicht aus, kreuzt diese aber noch nicht. Über einen Bereich von mehr als 1 m kann eindeutige Versetzungsaktivität in den Ti-Schichten im Bildaus- 


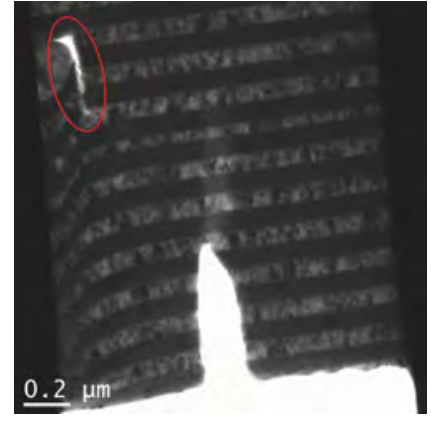

(a) BF vor 2. Verformung

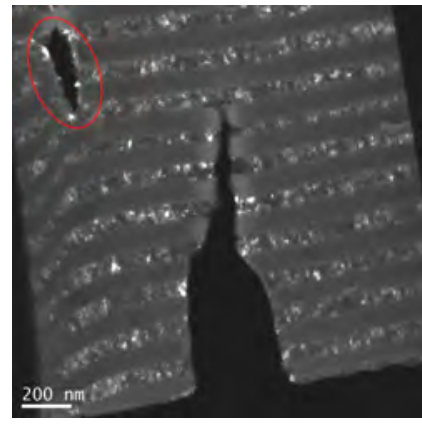

(b) DF-Übersicht

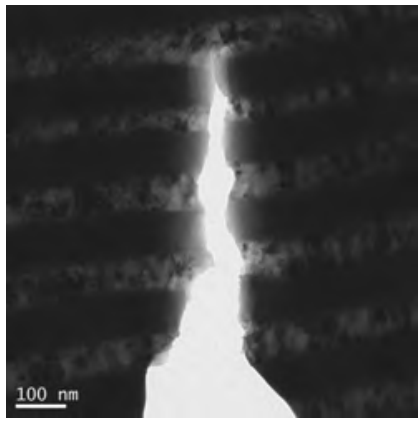

(c) BF Riss

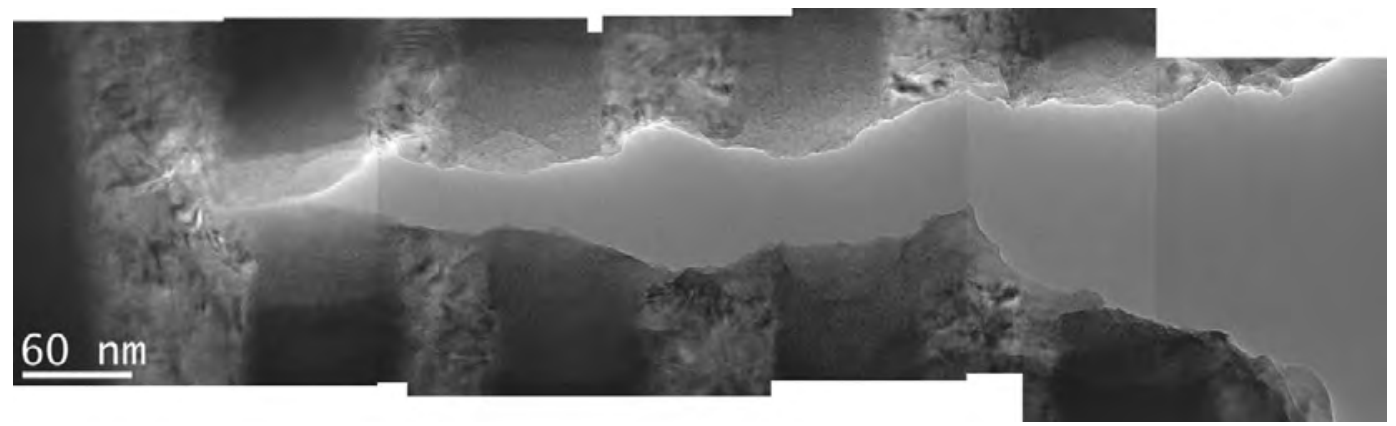

(d) Übersicht Riss

Abbildung 4.41: Bruchversuch Ti100/ZrO 2100 senkrecht zu den Grenzflächen Probe 1

schnitt festgestellt werden.

Im letzten Verformungsschritt dieses Bruchversuchs wächst der Riss insgesamt über drei weitere $\mathrm{ZrO}_{2}$ - und zwei Ti-Schichten weg von der Kerbe in die Lamelle hinein. In Abbildungen 4.41b und 4.41c sind eine Dunkel- und eine Hellfeldaufnahme der Lamelle am Ende des Bruchversuchs gezeigt. Der Riss hat sich senkrecht über die Grenzflächen hinweg in die jeweils benachbarte Schicht ausgebreitet und weist in beiden Materialien eine glatte Rissoberfläche auf. In Abbildung $4.41 b$ ist zusätzlich der Riss an dem Ti-Tropfen markiert. Dieser Riss hat sich im Verlauf der zweiten Verformung weiter geöffnet, ist aber nicht signifikant weiter gewachsen im Vergleich zur Risslänge am Ende der ersten Verformung.

Abbildung 4.41d zeigt eine zusammengesetzte Aufnahme von höher vergrößerten Einzelaufnahmen entlang der Rissoberfläche. Neben der glatten Oberfläche ist hier zu erkennen, dass der Riss bis in eine $\mathrm{ZrO}_{2}$-Schicht, jedoch nah zu der nächsten Grenzfläche, gewachsen ist.

\section{Ti100/ZrO $2100-$ Probe 2}

Eine weitere untersuchte Probe dieser Schichtdickenkombination ist in Abbildung 4.42a gezeigt. Anhand der HRTEM-Aufnahme in Abbildung 4.42b ist eindeutig zu erkennen, dass die Spitze der Kerbe in der Mitte einer $\mathrm{ZrO}_{2}$-Schicht liegt. Außerdem werden di- 
rekt an der Spitze der Kerbe kristalline Strukturen in der $\mathrm{ZrO}_{2}$-Schicht gefunden, die, wie bereits geschildert, durch die Wechselwirkung mit dem Ionenstrahl im FIB entstehen.

Dieser Bruchversuch ist in vier Verformungssegmente unterteilt. Während der ersten Bildserie können erste Versetzungsaktivitäten in Verbindung mit der Öffnung der Kerbe beobachtet werden. Die Versetzungsaktivität erstreckt sich über den gesamten Bildausschnitt, was einem Abstand von etwa $800 \mathrm{~nm}$ von der Spitze der Kerbe entspricht. Die Aktivität von Versetzungen ist auch im zweiten Verformungsschritt in vergleichbar großen Bereichen zu beobachten. Im Bereich direkt vor der Spitze der Kerbe ist die Aktivität jedoch erhöht. Zusätzlich ist eine deutliche Abrundung der Spitze zu erkennen, sodass am Ende der Bildserie die Spitze der Kerbe sehr nah an der Grenzfläche zur nächsten Ti-Schicht liegt. Ein Riss ist jedoch nicht zu erkennen.

Im dritten Schritt bildet sich ausgehend von der abgerundeten Spitze der Riss in die benachbarte Ti-Schicht. Die weiter folgenden Ti-Schichten zeigen eine deutlich erhöhte Versetzungsaktivität. Die $\mathrm{ZrO}_{2}$-Schichten haben in diesem Bereich einen helleren Kontrast in Dunkelfeldaufnahmen im Vergleich zu den $\mathrm{ZrO}_{2}$-Schichten weiter entfernt von dem Riss, was auf eine Einschnürung der $\mathrm{ZrO}_{2}$-Schichten schließen lässt. Am Ende des dritten Schrittes ist der Riss bis in die nächste Ti-Schicht gewachsen, was einer Risslänge von ca. $50 \mathrm{~nm}$ entspricht. Die weiteren Schichten, sowohl $\mathrm{ZrO}_{2}$ - als auch Ti-Schichten, sind noch nicht gerissen, jedoch in der jeweiligen Schichtebene stark verformt.

Im letzten Schritt der Verformung beginnt der Riss stärker zu wachsen. In Abbildungen 4.42c und 4.42d sind zwei Momentaufnahmen aus der Bildserie des letzten Verformungsschrittes gezeigt. In Abbildung 4.42c ist deutlich zu erkennen, dass der Riss bereits die $\mathrm{ZrO}_{2}$-Schicht erreicht hat (blauer Pfeil), obwohl die Ti-Schicht noch nicht komplett gerissen ist (roter Pfeil). In den weiteren Schichten (rote Markierung) ist zu beobachten, dass die $\mathrm{ZrO}_{2}$-Schichten wieder heller erscheinen als die weniger stark verformten Bereiche und sowohl die Ti- als auch die $\mathrm{ZrO}_{2}$-Schichten nicht gerissen sind. Im Bereich der Markierung ist eine deutlich stärkere Aktivität der Versetzungen im Verlauf dieses Verformungsschrittes zu beobachten.

In Abbildung 4.42d ist der Riss deutlich weiter gewachsen. Auch hier ist die Rissbrückenbildung in den Ti-Schichten offensichtlich. Die mit dem roten Pfeil markierte Schicht ist gerissen, wobei hingegen die mit den blauen Pfeilen markierten Ti-Schichten noch intakt sind, obwohl jeweils die benachbarten $\mathrm{ZrO}_{2}$-Schichten gerissen sind. Die Länge des Risses in den komplett gerissenen Schichten bis zur ersten mit einem blauen Pfeil markierten Ti-Schicht beträgt ca. $520 \mathrm{~nm}$.

Bevor die Rissoberfläche detailliert analysiert werden konnte, ist diese Lamelle durch eine unkontrollierte Bewegung der STM-Spitze komplett zerstört worden, sodass keine weiteren Untersuchungen möglich waren. 


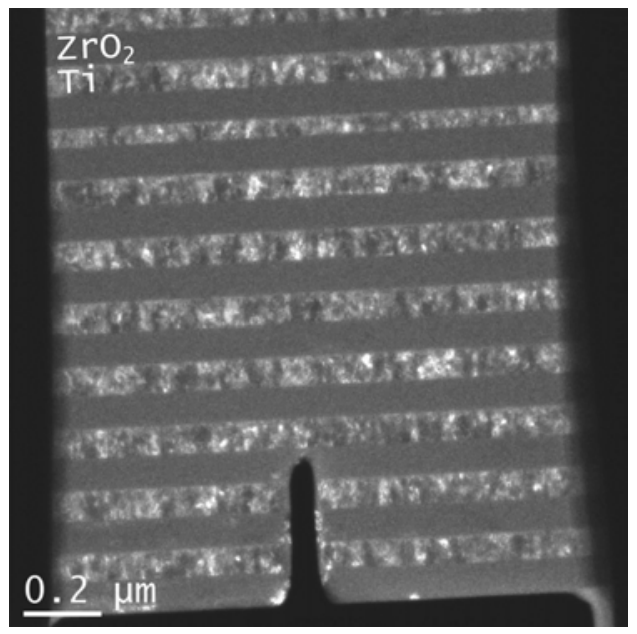

(a) DF-Übersicht

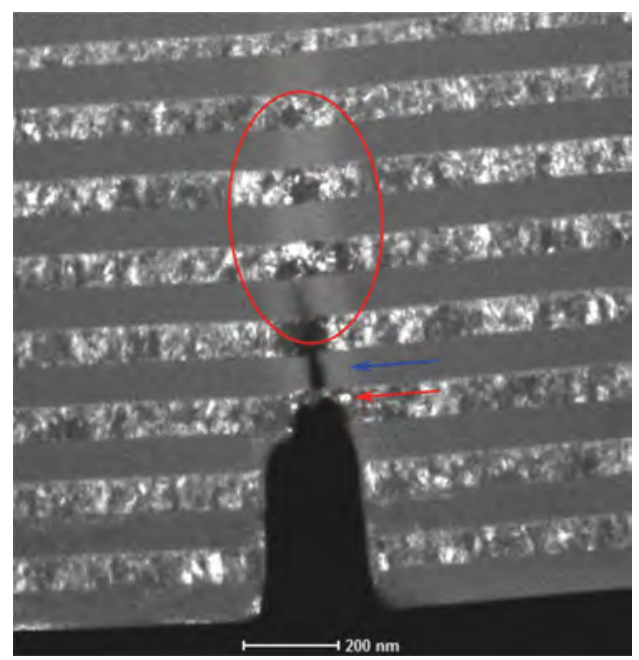

(c) Bild 150 4. Schritt

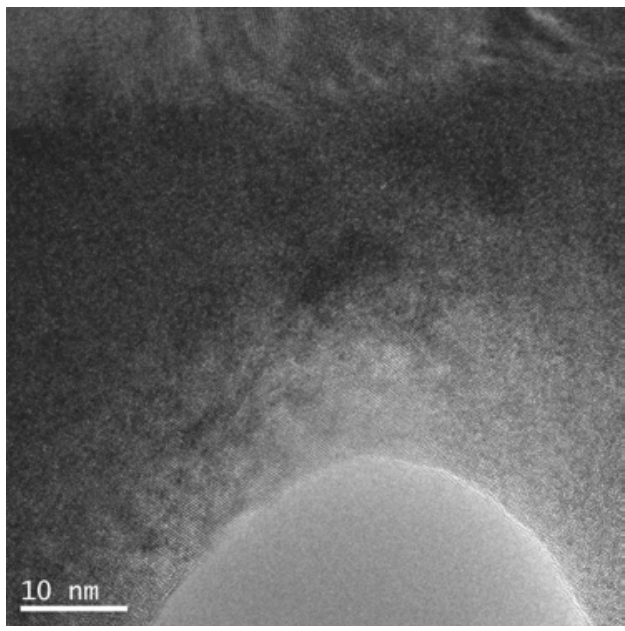

(b) HRTEM Kerbe

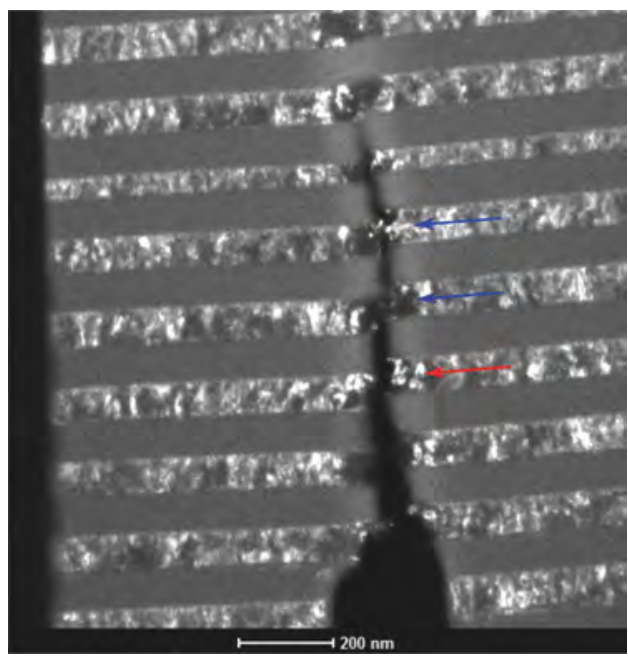

(d) Bild 300 4. Schritt

Abbildung 4.42: Bruchversuch Ti100/ZrO 100 senkrecht zu den Grenzflächen Probe 2

\section{Ti100/ZrO 2 100-Probe 3}

In Abbildung 4.43a ist eine Hellfeldaufnahme einer weiteren untersuchten Probe gezeigt. Die Spitze der Kerbe dieser Probe liegt in einer $\mathrm{ZrO}_{2}-\mathrm{Schicht}$ nur wenige Nanometer von der Grenzfläche zur nächsten Ti-Schicht entfernt (vgl. Abbildung 4.43b). Bei der Präparation ist die oberste $\mathrm{ZrO}_{2}$ - sowie die Hälfte der ersten Ti-Schicht mit dem FIB weggeschnitten worden, sodass die Multilage eine Doppellage dünner ist als die anderen Proben. Diese fehlenden Schichten haben aber im weiteren Verlauf des Bruchversuchs keinen Einfluss auf das Bruchverhalten.

Der Bruchversuch ist in nur zwei einzelne Verformungsschritte unterteilt, die beide in Hellfeldbildserien festgehalten werden. Im ersten Verformungsschritt ist neben der gewünschten Vergrößerung der Öffnungsweite der Kerbe auch die Versetzungsaktivität innerhalb der Ti-Schichten zu beobachten. Aufgrund der höheren Vergrößerung und 


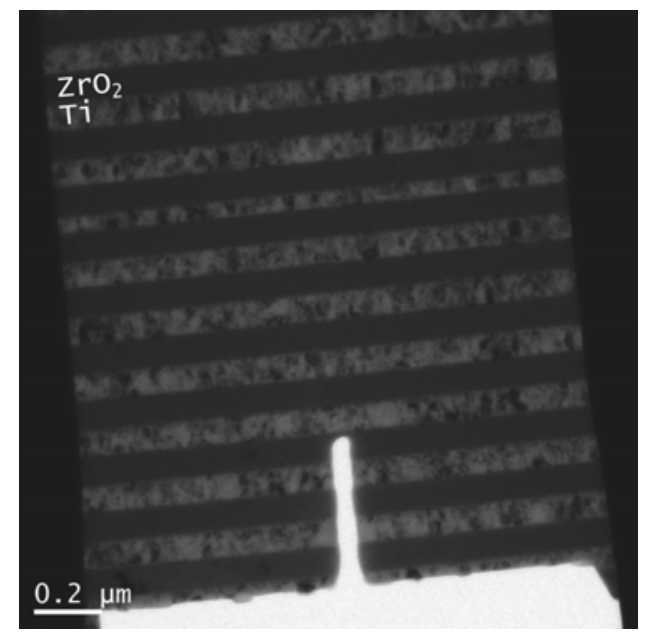

(a) BF-Übersicht

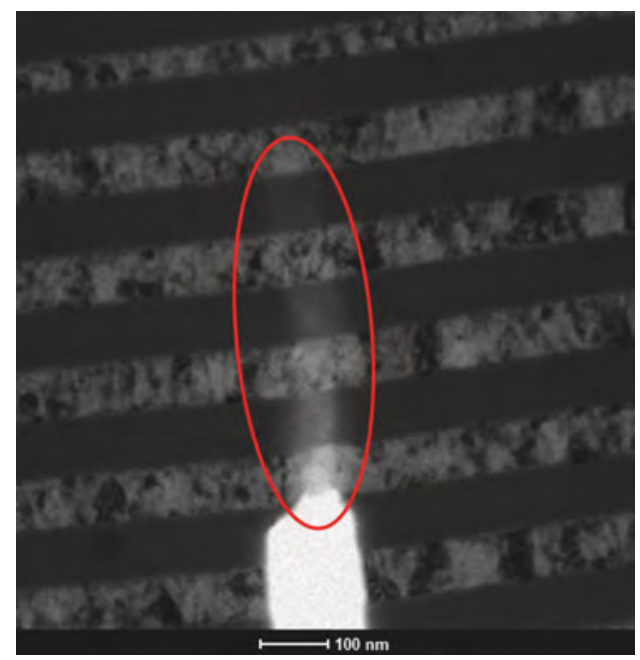

(c) Bild 160 2. Schritt

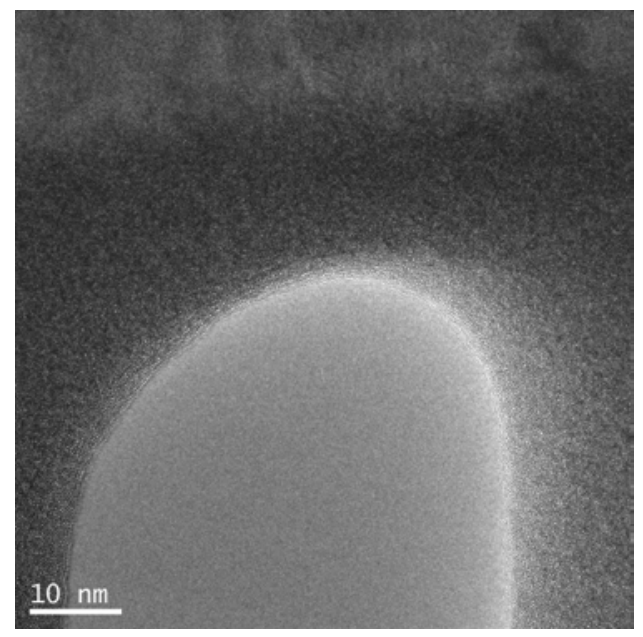

(b) HRTEM Kerbe

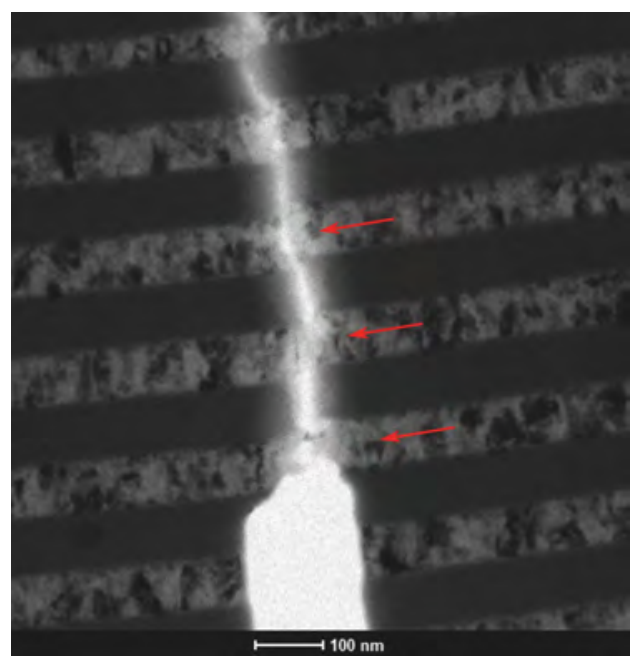

(d) Bild 240 2. Schritt

Abbildung 4.43: Bruchversuch Ti100/ZrO 100 senkrecht zu den Grenzflächen Probe 3

damit des kleineren Bildausschnittes kann hier eine Versetzungsaktivität nur bis zu einer Entfernung von ca. $700 \mathrm{~nm}$ beobachtet werden. Eine erhöhte Aktivität ist dabei in den beiden der Kerbe am nächsten liegenden Ti-Schichten zu erkennen. Ein Riss wird von der Spitze der Kerbe aus gebildet und wächst in Richtung der Grenzfläche zwischen der anfänglichen $\mathrm{ZrO}_{2}$-Schicht und der nächsten Ti-Schicht. Am Ende des ersten Schrittes liegt die Rissspitze genau an dieser Grenzfläche.

Im zweiten Verformungsschritt breitet sich der Riss in die der Kerbe am nächsten liegenden Ti-Schicht und die folgenden Schichten aus. Bevor die Schichten reißen, ist besonders in den $\mathrm{ZrO}_{2}$-Schichten wieder eine Einschnürung zu beobachten (hellerer Kontrast in Markierung in Abbildung 4.43c). Im weiteren Verlauf reißen die $\mathrm{ZrO}_{2}$-Schichten, obwohl die benachbarten Ti-Schichten zwar bereits stark verformt, aber noch nicht gerissen sind. In Abbildung 4.43d sind drei noch nicht gerissene Ti-Schichten markiert, die für den Riss eine Brücke in die nächste $\mathrm{ZrO}_{2}$-Schicht bilden. Im dynamischen Verhalten 


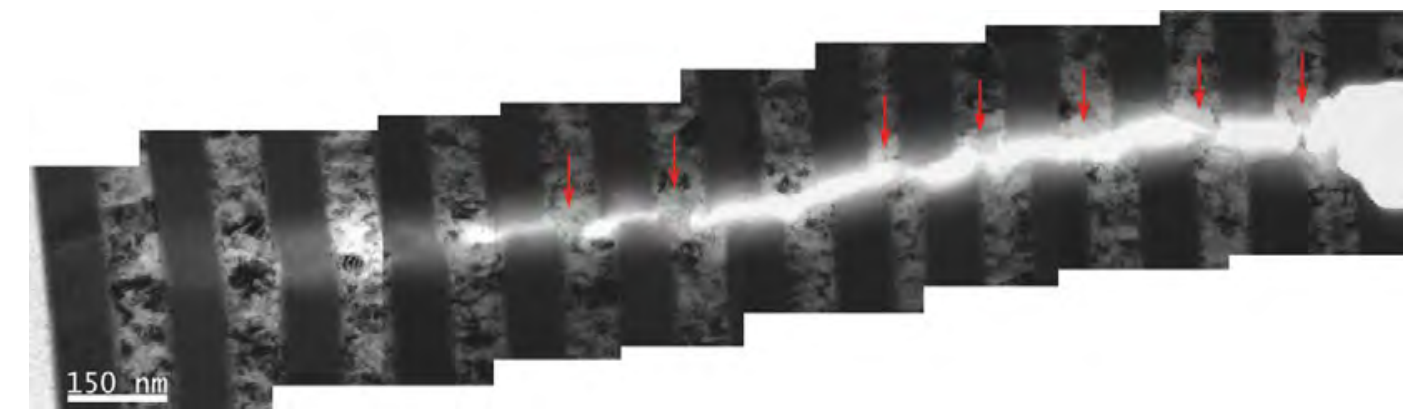

Abbildung 4.44: Bruchversuch Ti100/ZrO 100 senkrecht zu den Grenzflächen Probe 3 Übersicht Riss

dieses Verformungsschrittes ist zu beobachten, dass im Bereich der stark gedehnten TiRissbrücken eine signifikant höhere Versetzungsaktivität in Form von Kontrastwechseln herrscht. Am Ende des Bruchversuchs ist eine $\mathrm{ZrO}_{2}$-Schicht, mehr als $1 \mu \mathrm{m}$ entfernt von der ursprünglichen Spitze der Kerbe, die letzte gerissene Schicht.

In Abbildung 4.44 ist die zusammengesetzte Übersichtsaufnahme des gesamten Risses am Ende des zweiten Verformungsschrittes zu sehen. In dieser Abbildung sind die TiRissbrücken teilweise schon durch Relaxationsprozesse oder einer Driftbewegung der Spitze weiter verformt und letztlich gerissen. Die Positionen, an denen sicher eine Rissbrücke war oder noch vorhanden ist, sind in der Abbildung markiert. Abgesehen von den Positionen der Rissbrücken in den Ti-Schichten ist die Rissoberfläche sowohl im Ti als auch im $\mathrm{ZrO}_{2}$, wie schon in den vorher gezeigten Beispielen dieser Ti-Schichtdicke, glatt.

\section{Ti100/ZrO $2100-$ Probe 4}

Als letztes Beispiel der Bruchversuche der Ti-Schichtdicke von $d_{\mathrm{Ti}}=100 \mathrm{~nm}$ werden die Ergebnisse des Versuchs mit der in Abbildung 4.45a dargestellten Probe detailliert beschrieben. In der Hellfeldabbildung ist zu erkennen, dass die $\mathrm{ZrO}_{2}$-Schichten ausschließlich im Bereich der Kerbe teilweise kristalline Strukturen aufweisen. Jedoch ist der Bereich vor der Spitze der Kerbe nicht kristallin (vgl. HRTEM-Aufnahme in Abbildung 4.45b). Zur Verdeutlichung ist die Grenzfläche zwischen den beiden Schichten markiert.

Dieses Experiment ist in zwei Verformungssequenzen aufgeteilt. Innerhalb der ersten Sequenz öffnet sich wie gewünscht die Kerbe durch die angelegte Verformung. Zusätzlich kann in der Hellfeldbildserie die Bewegung der Biegekonturen, bevorzugt in den Ti-Körnern der verschiedenen Ti-Schichten, im gewählten Bildausschnitt beobachtet werden. Aber auch im Bereich der kristallinen $\mathrm{ZrO}_{2}$-Schichten in direkter Umgebung der Kerbe sind Biegekonturen eindeutig zu erkennen. Es bildet sich weder ein Riss in der Lamelle, noch kann eine Kontraständerung des Materials an der Spitze der Kerbe festgestellt werden. 


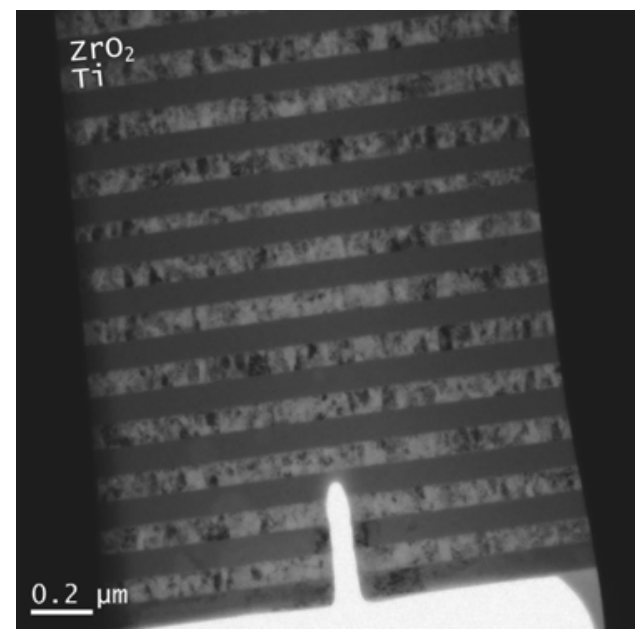

(a) BF-Übersicht

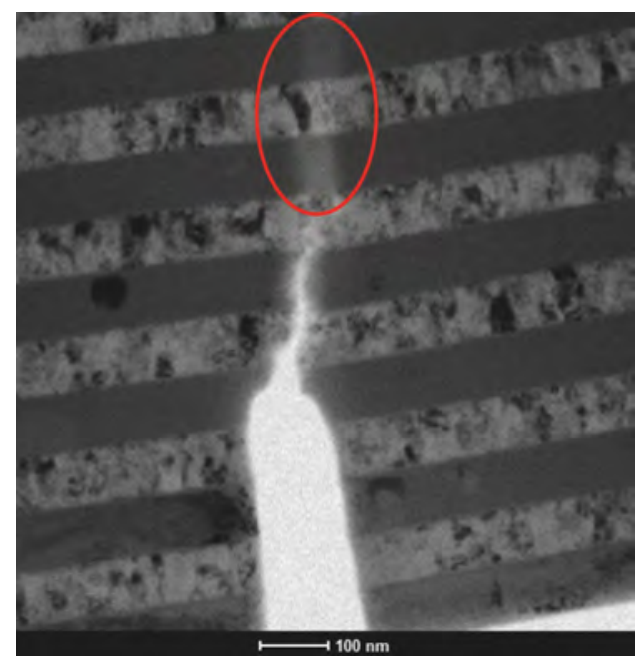

(c) Bild 330 2. Schritt

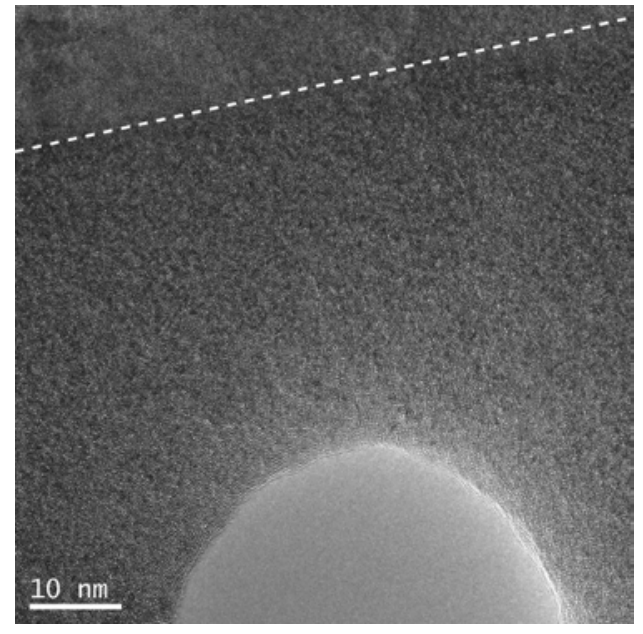

(b) HRTEM Kerbe

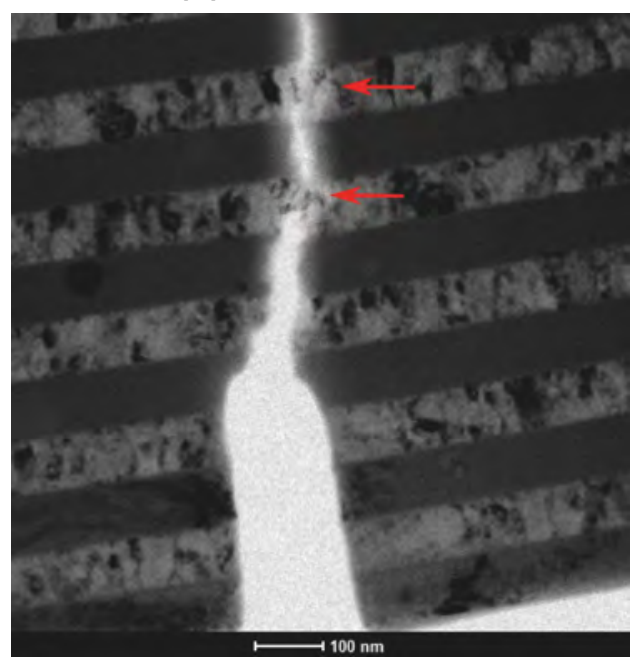

(d) Bild 460 2. Schritt

Abbildung 4.45: Bruchversuch Ti100/ZrO 100 senkrecht zu den Grenzflächen Probe 4:(c) und (d) zeigen zwei Momentaufnahmen des zweiten Verformungsschrittes

In der zweiten Verformungssequenz hingegen bildet sich der Riss ausgehend von der Spitze der Kerbe in der amorphen $\mathrm{ZrO}_{2}$-Schicht. Der Riss breitet sich leicht nach rechts abgelenkt aus (vgl. Abbildung 4.45c). Während der gesamten Verformung sind schnelle Kontrastwechsel über den gesamten Bildausschnitt zu beobachten. Aufgrund der gewählten Vergrößerung im Mikroskop beträgt daher die Entfernung der Positionen der Kontrastwechsel von der Spitze der Kerbe bzw. des Risses mindestens $570 \mathrm{~nm}$.

Bevor der Riss eine der $\mathrm{ZrO}_{2}$-Schichten durchquert, ist zu beobachten, dass sich der Kontrast in der jeweiligen $\mathrm{ZrO}_{2}$-Schicht erhellt. Das spricht dafür, dass die $\mathrm{ZrO}_{2^{-}}$ Schichten dünner werden bevor sie reißen und das in Ausbreitungsrichtung des Elektronenstrahls. Abbildung $4.45 \mathrm{c}$ zeigt eine Momentaufnahme der zweiten Verformungssequenz. Hier ist im Bereich der roten Markierung die Verformung der $\mathrm{ZrO}_{2}$-Schichten zu erkennen. Die zweite und dritte Ti-Schicht, die im Verlauf des Bruches von dem 


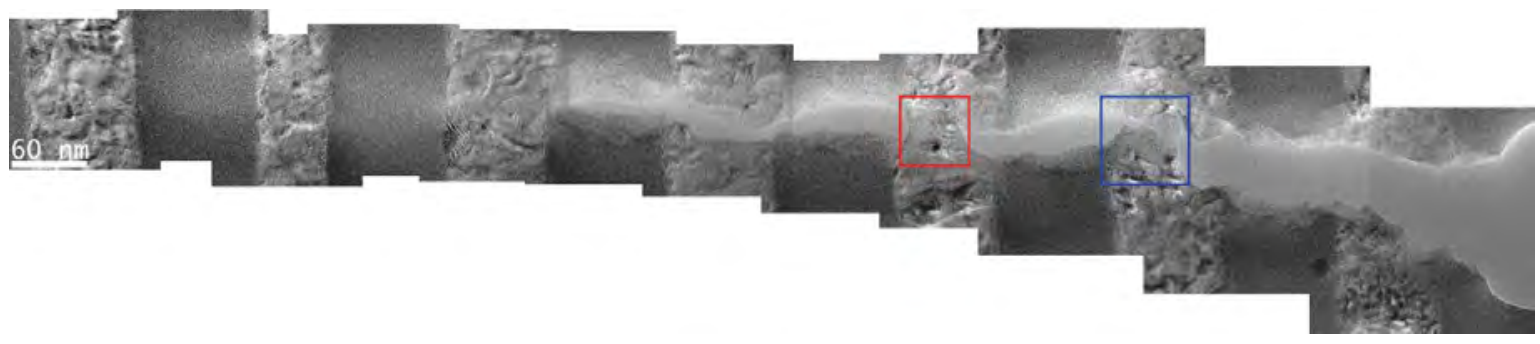

(a) Übersicht Riss

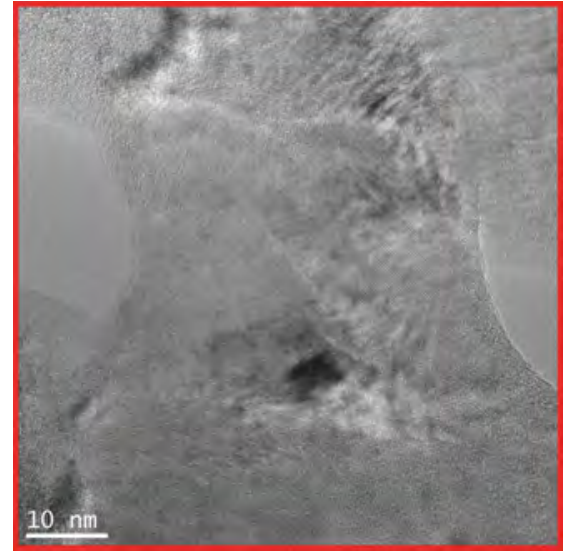

(b) Rote Markierung

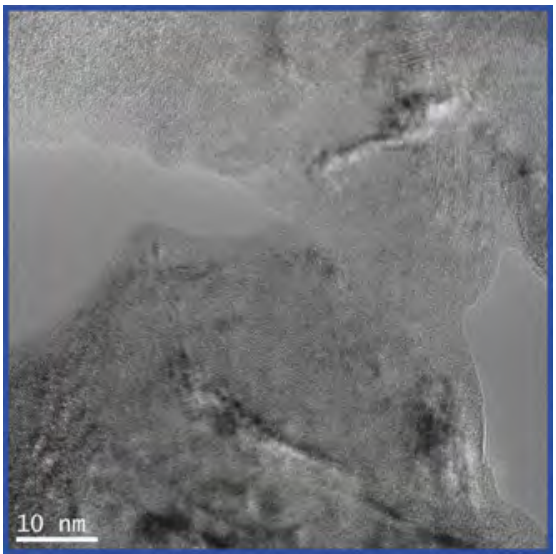

(c) Blaue Markierung

Abbildung 4.46: Bruchversuch Ti100/ZrO 100 senkrecht zu den Grenzflächen Probe 4 Riss

Riss durchquert werden, sind in Abbildung 4.45d mit Pfeilen versehen. Diese beiden Ti-Schichten zeigen die in den vorherigen Bruchversuchen bereits beobachtete Rissüberbrückung. Dieser Bruchversuch bestätigt weiterhin die erhöhte Versetzungsaktivität im Bereich der Rissüberbrückung.

In Abbildung 4.46a ist die zusammengesetzte Übersichtsaufnahme des Risses am Ende des zweiten Verformungsschrittes gezeigt. Die gesamte Risslänge beträgt ca. $700 \mathrm{~nm}$, wobei der Riss acht Schichten komplett durchquert hat. Es kann zusätzlich beobachtet werden, dass die nächsten zwei $\mathrm{ZrO}_{2}$-Schichten vor der Rissspitze (links in Abbildung 4.46a bereits verformt sind, obwohl die eigentliche Rissspitze mehrere hundert Nanometer entfernt ist. Weiterhin sind auch hier die beiden Stellen der Rissüberbrückung in den Ti-Schichten zu sehen. In Abbildungen 4.46b und 4.46c sind höher aufgelöste Aufnahmen im Bereich der Rissüberbrückung (Markierungen in Abbildung 4.46a) dargestellt. Diese beiden hochaufgelösten Aufnahmen zeigen eindeutig, dass die Ti-Schichten noch nicht gerissen sind. In diesem stark verformten Bereich können zusätzlich kristalline Strukturen beobachtet werden.

\section{Zusammenfassung $\mathrm{Ti} 100 / \mathrm{ZrO}_{2} 100$}

Die Bruchversuche des Ti100/ZrO 100 -Schichtdickenverhältnisses senkrecht zu den Grenzflächen zeigen für alle durchgeführten Experimente ein vergleichbares Verhalten. 
Der Riss wird, wie in der Geometrie der Bruchversuche entlang der Grenzfläche, an der Spitze der Kerbe gebildet. Von dort ausgehend wächst der Riss kontrollierbar über alle Grenzflächen hinweg in Richtung des Si-Substrates. Die Ausbreitungsrichtung liegt senkrecht zu den Grenzflächen. Während der Rissausbreitung werden Rissbrücken in den Ti-Schichten ausgebildet, was bedeutet, dass im Bereich der Rissbrücken eine stark lokalisierte plastische Verformung vorliegt. In drei von fünf Bruchversuchen kann die Rissüberbrückung über die $\mathrm{ZrO}_{2}$-Schichten beobachtet werden. 


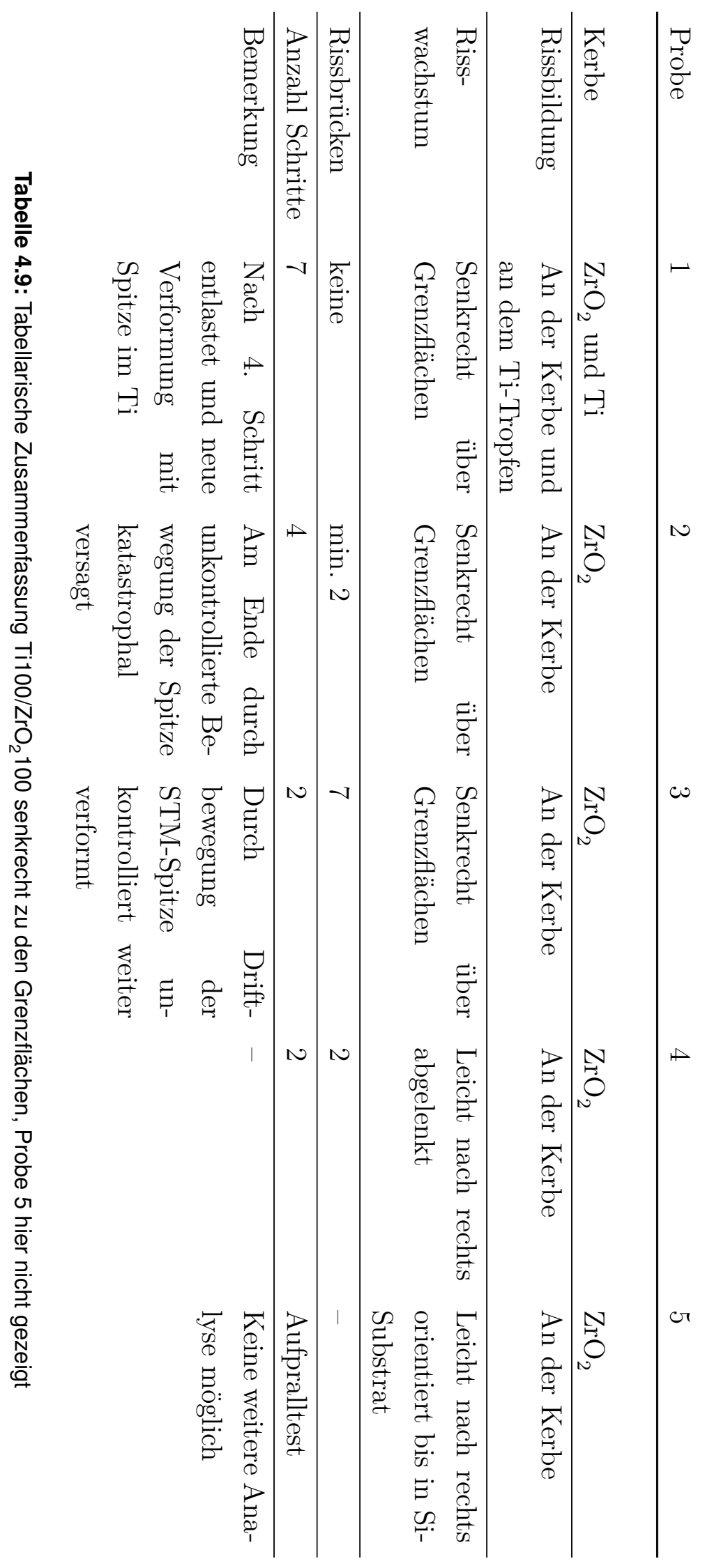




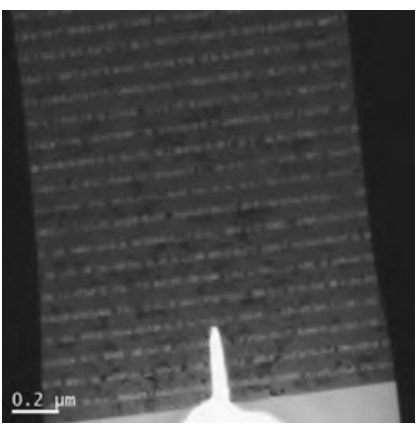

(a) BF Übersicht

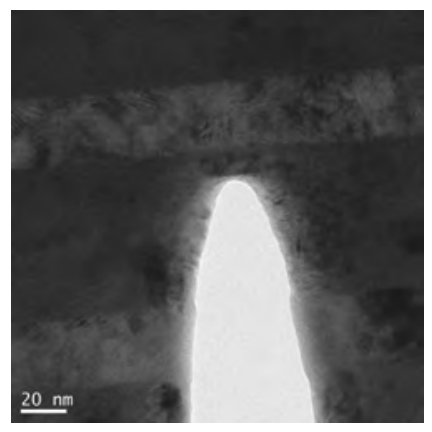

(b) BF Kerbe

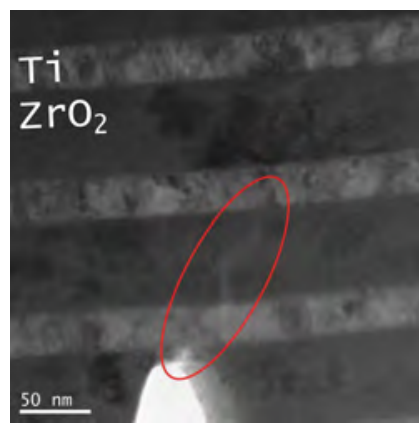

(c) Riss nach 6. Schritt

Abbildung 4.47: Bruchversuch $\mathrm{Ti} 50 / \mathrm{ZrO}_{2} 100$ senkrecht zu den Grenzflächen Probe 1

\subsection{2 $\mathrm{Ti50} / \mathrm{ZrO}_{2} 100$}

$\mathrm{Ti50} / \mathrm{ZrO}_{2} 100-$ Probe 1

In Abbildung 4.47a ist eine Hellfeldübersichtsaufnahme der ersten Probe mit einer dünneren Schichtdicke von $d_{\mathrm{Ti}}=50 \mathrm{~nm}$ gezeigt. In den dunkleren Bereichen der $\mathrm{ZrO}_{2^{-}}$ Schichten können mit weiteren HRTEM-Aufnahmen (hier nicht gezeigt) kristalline Strukturen nachgewiesen werden. Zusätzlich ist in dieser Aufnahme noch ein Teil der Si-Deckschicht auf der obersten $\mathrm{ZrO}_{2}$-Schicht zu erkennen (helle Schicht unten in Abbildung 4.47a). Die Kerbe dieser Probe ist in Abbildung 4.47b zu sehen. Die Spitze der Kerbe endet in einer $\mathrm{ZrO}_{2}$-Schicht ca. $20 \mathrm{~nm}$ von der Grenzfläche zur nächsten TiSchicht entfernt. Auch hier können kristalline Stellen im $\mathrm{ZrO}_{2}$ beobachtet werden.

Die Probe wird insgesamt zehnmal verformt. In den ersten vier Verformungsschritten sind lediglich die Bewegungen von Biegekonturen in sowohl den $\mathrm{ZrO}_{2}$ - als auch den Ti-Schichten und eine Vergrößerung der Öffnungsweite der Kerbe zu erkennen. Am Ende des vierten Schrittes ist eine Stelle helleren Kontrastes in der Größenordnung von wenigen Nanometern an der Spitze der Kerbe zu sehen. Ein Riss ist aber noch nicht eindeutig zu erkennen. Im fünften Schritt bildet sich an der Kerbe an der bereits verformten Stelle ein Riss, der aber nur wenige Nanometer lang ist. In der angrenzenden Ti-Schicht kann eine erhöhte Versetzungsaktivität festgestellt werden. Im sechsten Verformungsschritt ist neben dem Riss an der Kerbe zusätzlich eine verformte Stelle in der nächsten $\mathrm{ZrO}_{2}$-Schicht zu sehen (vgl. Markierung 4.47c). Diese Stelle entsteht, ohne dass die Ti-Schicht zwischen den beiden verformten $\mathrm{ZrO}_{2}$-Schichten signifikant gedehnt ist.

In den letzten vier Verformungsschritten dieses Bruchversuchs versagt die Probe letztlich katastrophal. In Abbildung 4.48 sind drei Hellfeldaufnahmen nach den jeweils angegebenen Verformungsschritten gezeigt, um die Entwicklung des Risses nachzuvollziehen. Im siebten Verformungsschritt wächst der Riss an der Spitze der Kerbe in Richtung der Grenzfläche zu der nächsten Ti-Schicht (schwarzer Pfeil in Abbildung 


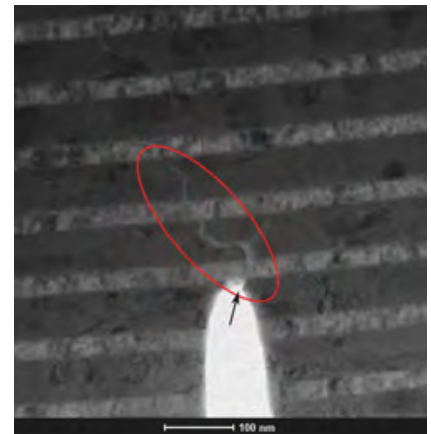

(a) Riss nach 7. Schritt

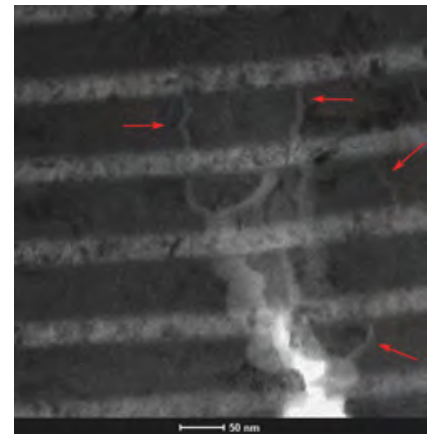

(b) Riss nach 9. Schritt

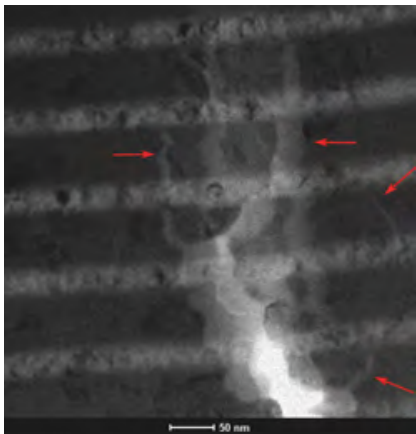

(c) Riss nach 10. Schritt

Abbildung 4.48: Bruchversuch $\mathrm{Ti50} / \mathrm{ZrO}_{2} 100$ senkrecht zu den Grenzflächen Probe 1 Riss

4.48a). Bevor die Ti-Schicht jedoch reißt, verformt sich diese ausgehend von der bereits geschädigten Stelle in der nächsten $\mathrm{ZrO}_{2}$-Schicht weiter. Auch die übernächste $\mathrm{ZrO}_{2}$-Schicht wird verformt (siehe rote Markierung in Abbildung 4.48a). Die im siebten Schritt neu entstandenen Verformungen im $\mathrm{ZrO}_{2}$ sind im Gegensatz zu der ersten Verformung der $\mathrm{ZrO}_{2}$-Schicht im sechsten Schritt, nach links orientiert. In allen TiSchichten im Bildausschnitt können Versetzungsaktivitäten beobachtet werden.

Im achten und neunten Verformungsschritt bildet sich die komplexe Rissstruktur aus, wie sie in Abbildung 4.48b zu sehen ist. Mit den roten Pfeilen sind die Stellen markiert, an denen die $\mathrm{ZrO}_{2}$-Schichten bereits verformt sind. Auch in diesen beiden Verformungsschritten sind die Versetzungsaktivitäten über den gesamten Bildausschnitt verteilt, wobei in der Nähe des Risses oder der verformten Stellen in den $\mathrm{ZrO}_{2}$-Schichten die Aktivität in den Ti-Schichten deutlich erhöht ist. Am Ende des neunten Schrittes hat der Riss eine Ti- und eine $\mathrm{ZrO}_{2}$-Schicht durchquert.

Im letzten Schritt versagt die Probe letztlich. In Abbildung $4.48 \mathrm{c}$ ist die letzte Aufnahme der Bildserie des letzten Verformungsschrittes gezeigt. Im Vergleich zu der Konfiguration am Ende des neunten Schrittes ist zu beobachten, dass sich der Riss weiter ausgebreitet hat, bevor das katastrophale Versagen eintritt. In den Momentaufnahmen vor dem Bruch der Probe kann eine deutlich ausgeprägte Versetzungsaktivität in der Nähe des Risses festgestellt werden.

\section{$\mathrm{Ti50} / \mathrm{ZrO}_{2} 100-$ Probe 2}

Der Bruchversuch an der in Abbildung 4.49a dargestellten Probe ist in vier einzelne Verformungssegmente unterteilt. Zu Beginn des Versuchs liegt die Spitze der Kerbe in einer $\mathrm{ZrO}_{2}$-Schicht (siehe Abbildung 4.49b). Die helle Schicht, zu erkennen als Saum um den Rand der Kerbe, besteht aus redeponiertem Material, welches sich im FIB während der Bearbeitung der Lamelle mit dem Ionenstrahl an dieser Stelle wieder angelagert hat. Diese zusätzliche Schicht im Bereich der Kerbe hat jedoch keinen Einfluss 


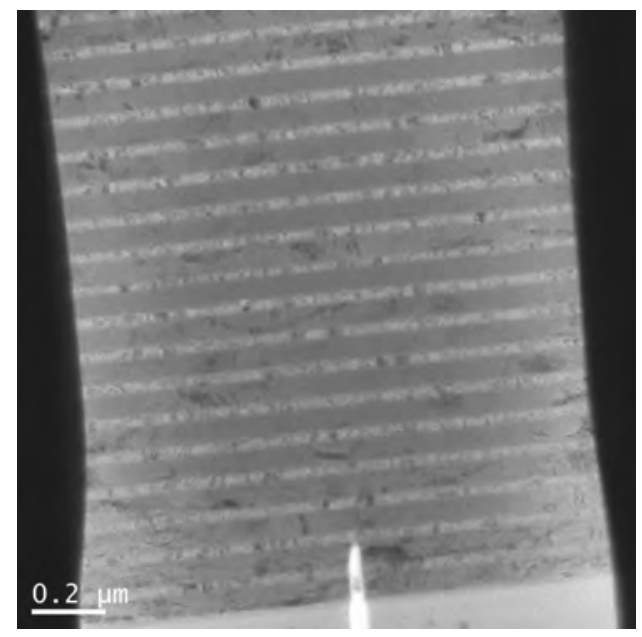

(a) BF Übersicht

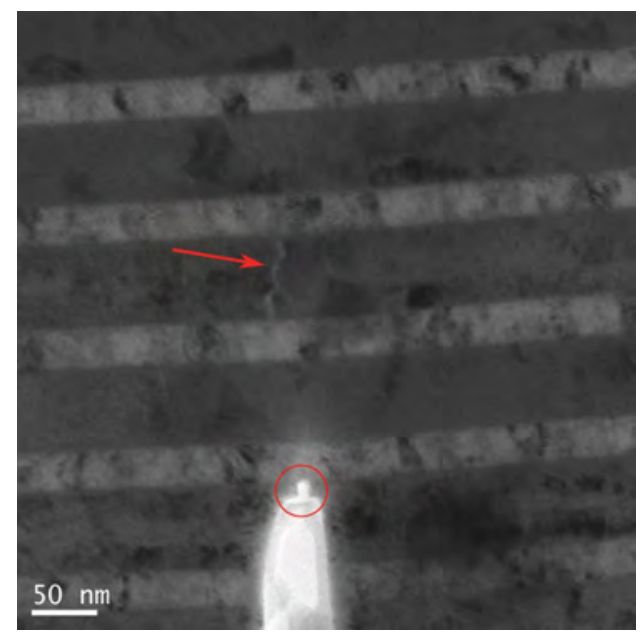

(c) Riss nach 3. Schritt

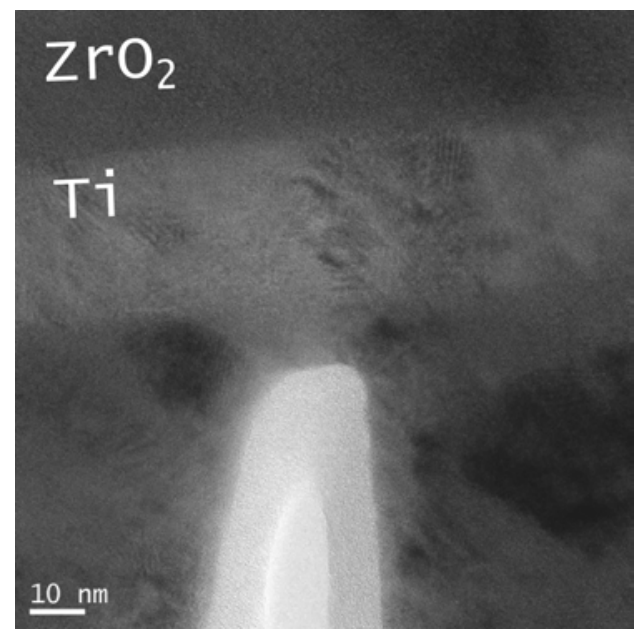

(b) BF Kerbe

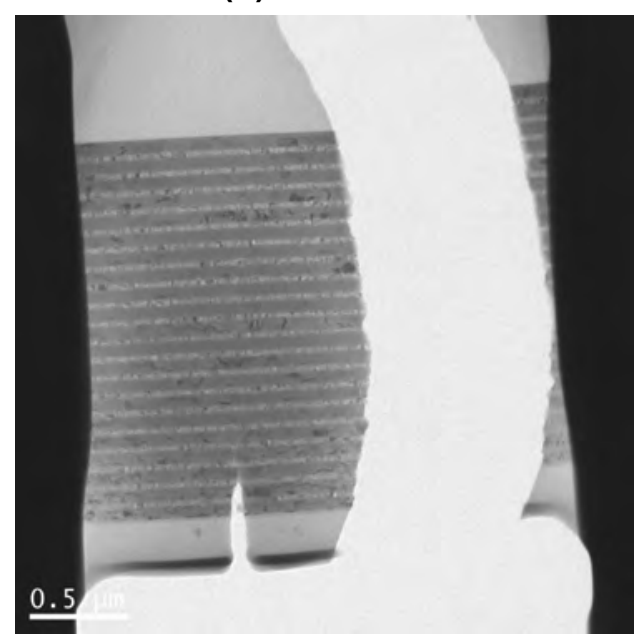

(d) BF Übersicht Ende

Abbildung 4.49: Bruchversuch $\mathrm{Ti} 50 / \mathrm{ZrO}_{2} 100$ senkrecht zu den Grenzflächen Probe 2

auf das Bruchverhalten.

Im ersten und zweiten Verformungssegment dieses Bruchversuchs ist eine Bewegung der Biegekonturen in beiden Materialien zu beobachten. $\mathrm{Zu}$ einer Rissbildung kommt es in diesen Verformungsschritten noch nicht. Die Öffnungsweite der Kerbe vergrößert sich jedoch kontinuierlich.

Im dritten Verformungsschritt bildet sich an der Spitze der Kerbe ein Riss in der $\mathrm{ZrO}_{2}$-Schicht, der sich in Richtung der nächsten Ti-Schicht bewegt. Sowohl diese Rissbildung als auch die darauf folgende Bewegung ist verknüpft mit einer ausgeprägten Versetzungsaktivität in der Nähe des Risses. Über den gesamten Bildausschnitt verteilt sind ebenfalls schnelle Kontrastwechsel zu beobachten. Am Ende des dritten Schrittes ist der Riss bis zur Grenzfläche zur nächsten Ti-Schicht gewachsen (siehe Abbildung 4.49c). Zusätzlich zu diesem Riss ist eine weitere Stelle in Abbildung 4.49c markiert, an der das $\mathrm{ZrO}_{2}$ verformt ist. 


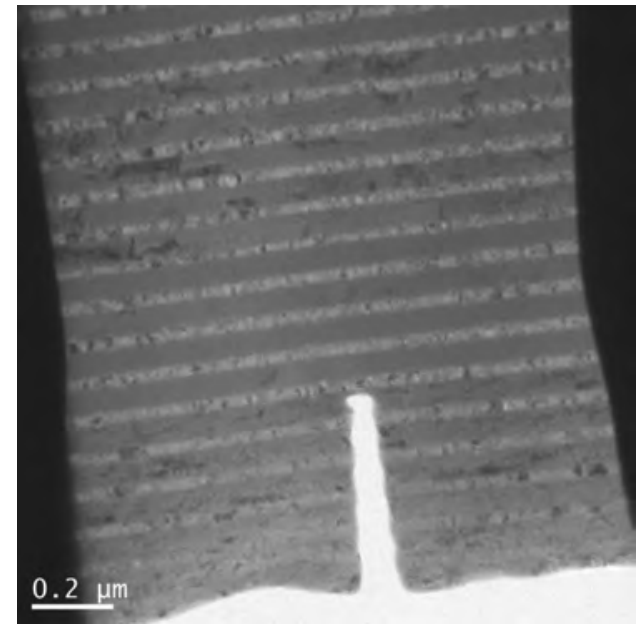

(a) BF Übersicht

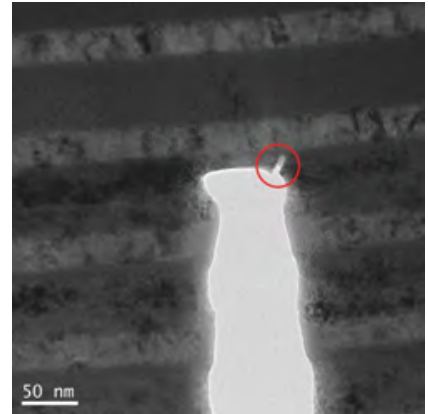

(c) Riss nach 2. Schritt

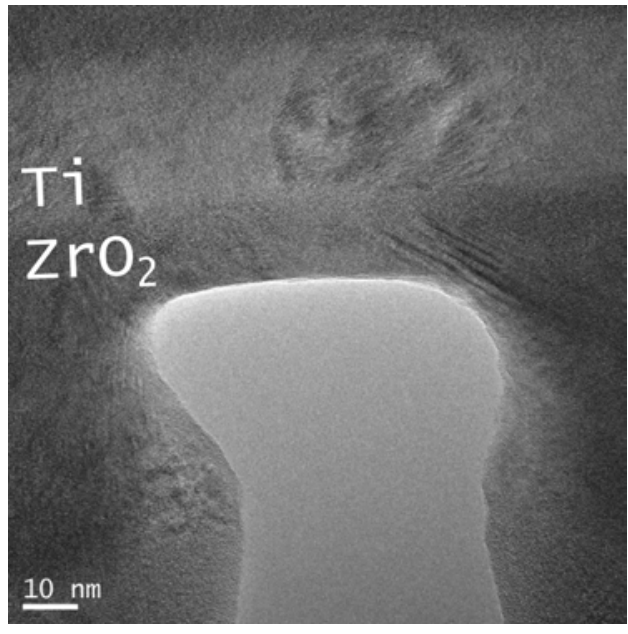

(b) HRTEM Kerbe

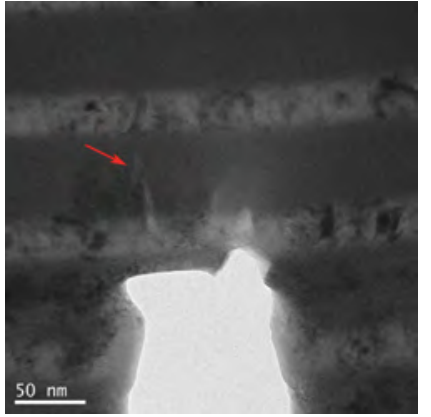

(d) Riss nach 5. Schritt

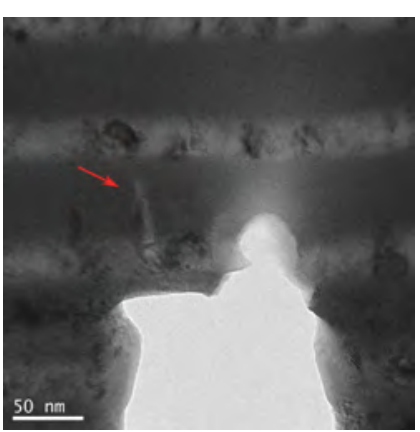

(e) Riss nach 7. Schritt

Abbildung 4.50: Bruchversuch $\mathrm{Ti} 50 / \mathrm{ZrO}_{2} 100$ senkrecht zu den Grenzflächen Probe 3

Im letzten Verformungssegment wächst der Riss von der Kerbe aus in die Ti- und die darauf folgende $\mathrm{ZrO}_{2}$-Schicht, wobei die Ti-Schicht noch nicht gerissen ist und daher wieder den Riss in die $\mathrm{ZrO}_{2}$-Schicht überbrückt. Nach diesem Verformungsschritt reißt die Probe entlang eines Risses, der sich außerhalb des Bildausschnittes gebildet hat, über alle Grenzflächen hinweg auseinander.

\section{$\mathrm{Ti50} / \mathrm{ZrO}_{2} 100-$ Probe 3}

Als letztes Beispiel der Bruchversuche an den Proben mit einer $50 \mathrm{~nm}$ dicken Ti-Schicht wird das Bruchverhalten der Probe in Abbildung 4.50 detailliert beschrieben. Die Spitze der Kerbe liegt für diese Probe in einer $\mathrm{ZrO}_{2}$-Schicht (vgl. Abbildung 4.50b). Der Bruchversuch dieser Probe ist in sieben Verformungsschritte unterteilt. Im ersten Schritt bildet sich noch kein Riss, jedoch ist eine Bewegung der Biegekonturen in beiden Schichten zu beobachten.

Im zweiten Schritt entsteht ein Riss an der Kerbe (vgl. Abbildung 4.50c). Dieser Riss wächst in den folgenden zwei Schritten leicht nach rechts orientiert in Richtung der ersten Grenzfläche zur nächsten Ti-Schicht. Innerhalb dieser Ti-Schicht ist im Bereich 
des Risses eine erhöhte Aktivität von Versetzungen zu beobachten. Es können zusätzlich Versetzungsaktivitäten in allen Ti-Schichten im Bildausschnitt registriert werden. Im fünften Schritt verbreitert sich der Riss, wächst aber nicht über die Grenzfläche in die Ti-Schicht. Es bildet sich jedoch eine verformte Stelle in der nächsten $\mathrm{ZrO}_{2}$-Schicht (Pfeil in Abbildung 4.50d). Diese Stelle liegt im Bereich dunkleren Kontrastes in der $\mathrm{ZrO}_{2}$-Schicht, sodass davon ausgegangen werden kann, dass das Material hier kristallin ist.

Im sechsten Verformungsschritt wächst der Riss letztlich bis in die erste Ti-Schicht nach der Kerbe. Während des gesamten Verformungsschrittes sind Versetzungsaktivitäten über den Bildausschnitt verteilt. Im Bereich des Risses ist die nächste $\mathrm{ZrO}_{2}$-Schicht eingeschnürt, was sich anhand des helleren Kontrastes feststellen lässt.

Im letzten Verformungsschritt, in dem der maximale Verfahrweg der STM-Spitze erreicht wird, verbreitert sich der Riss und bewegt sich in die bereits verformte $\mathrm{ZrO}_{2^{-}}$ Schicht. Weiterhin zeigt die $\mathrm{ZrO}_{2}$-Schicht im Bereich vor der eigentlichen Rissspitze einen helleren Kontrast (vgl. Markierung in Abbildung 4.50e). Am Ende des Bruchversuchs beträgt die erreichte Risslänge hier nur etwa $50 \mathrm{~nm}$.

\section{Zusammenfassung $\mathrm{Ti} 50 / \mathrm{ZrO}_{2} 100$}

Im Fall der $50 \mathrm{~nm}$ dicken Ti-Schichten breitet sich der Riss, wie schon für die dickeren Ti-Schichten, senkrecht zu den Grenzflächen aus. Der Riss wird an der Spitze der Kerbe gebildet. Das Bruchverhalten ist in der Art leicht verändert im Vergleich zu den Ti100/ZrO 100 -Proben, indem weniger Risswachstum aber auch katastrophales Versagen beobachtet werden kann. Außerdem wird in einem Fall an einigen Stellen in den $\mathrm{ZrO}_{2}$-Schichten eine Verformung festgestellt. Weiterhin wird in allen Bruchversuchen nur eine einzige Rissbrücke gefunden, die bei den dicken Ti-Schichten deutlich häufiger auftritt. 


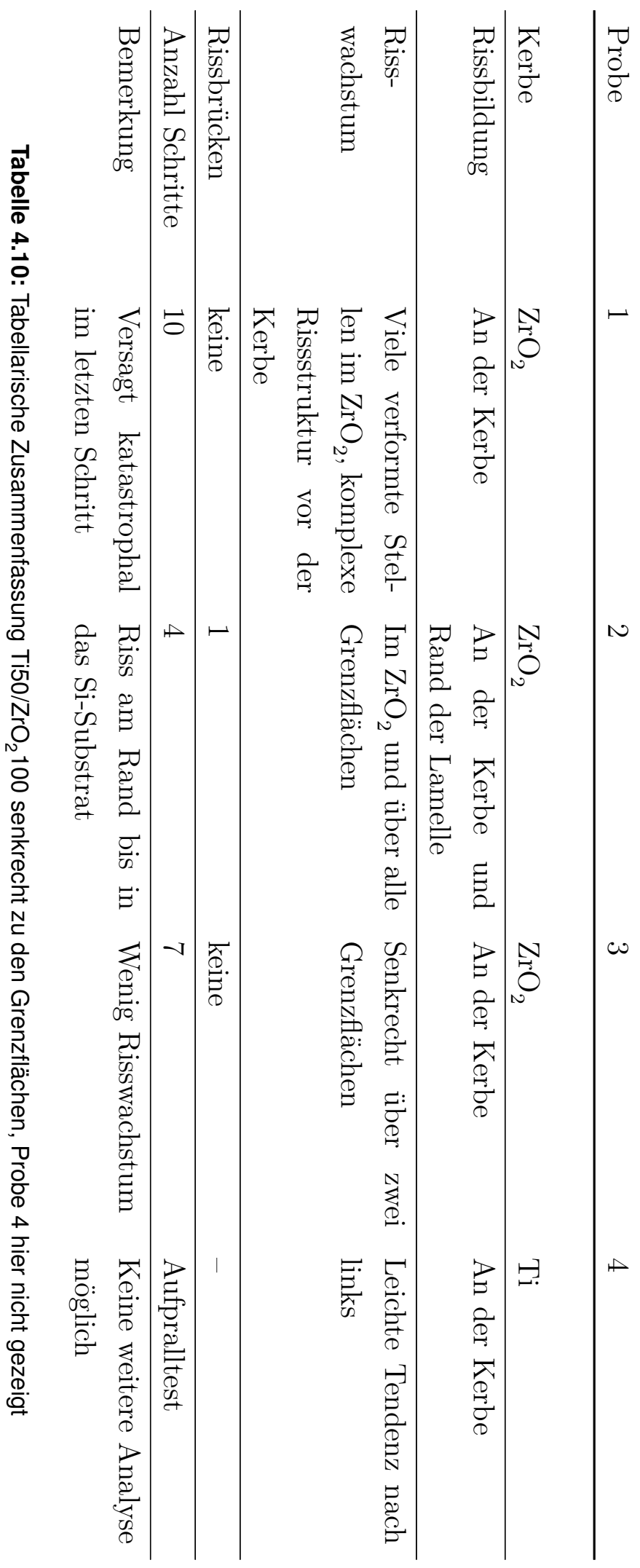




\subsection{3 $\mathrm{Ti} 10 / \mathrm{ZrO}_{2} 100$}

\section{Ti10/ZrO 100 -Probe 1}

In Abbildung 4.51a ist eine Hellfeldübersichtsaufnahme der ersten Probe der geringsten Ti-Schichtdicke von $d_{\mathrm{Ti}}=10 \mathrm{~nm}$ gezeigt. Für die $\mathrm{ZrO}_{2}$-Schichten ist auffällig, dass es vergleichsweise viele Stellen dunkleren Kontrastes gibt. An diesen Stellen können mit HRTEM-Aufnahmen wiederum kristalline Strukturen in den $\mathrm{ZrO}_{2}$-Schichten nachgewiesen werden. Die dunklere Schicht am oberen Ende der Lamelle (unten in Abbildung 4.51a ist die Si-Deckschicht, die im FIB nicht komplett entfernt wurde. Eine vergrößerte Aufnahme der Spitze der Kerbe ist in Abbildung 4.51b zu sehen. Die Position der Spitze liegt in einer $\mathrm{ZrO}_{2}$-Schicht. Eine HRTEM-Aufnahme an der Spitze der Kerbe zeigt, dass dort keine kristallinen Strukturen vorhanden sind, sodass der Riss in einer amorphen $\mathrm{ZrO}_{2}$-Schicht gebildet werden muss.

Der Bruchversuch ist in zwei Verformungsschritte unterteilt. Abbildung 4.51c zeigt die letzte Aufnahme der Bildserie des ersten Schrittes. Während dieses Verformungsschrittes öffnet sich die Kerbe kontinuierlich und sowohl in den stellenweise kristallinen $\mathrm{ZrO}_{2}-$ als auch in den dünnen Ti-Schichten können Biegekonturen beobachtet werden. Zum Ende dieser Verformung bildet sich der markierte Bereich helleren Kontrastes vor der Spitze der Kerbe aus (vgl. Markierung in Abbildung 4.51c). Die Aktivität von Versetzungen in den Ti-Schichten kann in dieser Bildserie nicht eindeutig festgestellt werden. In Abbildungen 4.51d bis 4.51f sind drei Momentaufnahmen der Bildserie des zweiten Verformungsschrittes gezeigt. Während dieses Schrittes bildet sich an der bereits lokal verformten Stelle im $\mathrm{ZrO}_{2}$ ein Riss, der leicht nach rechts abgelenkt in Richtung der nächsten Grenzfläche wächst. Zusätzlich breitet sich der Bereich helleren Kontrastes über die nächsten drei $\mathrm{ZrO}_{2}$-Schichten aus. Einige Aufnahmen weiter wächst der Riss kontinuierlich über weitere Grenzflächen hinweg, bis er innerhalb zweier Einzelaufnahmen katastrophal bis in das Si-Substrat wächst und die Lamelle komplett durchreißt. Es ist eindeutig zu erkennen, wie sich mit dem Riss auch der hellere Bereich durch die Lamelle bewegt. Bevor der Riss unkontrolliert wächst, kann eine Risslänge von ca. 200 nm gemessen werden. Während des Risswachstums bzw. des katastrophalen Versagens kann die Bewegung von Biegekonturen in beiden Materialien beobachtet werden. In Abbildung 4.52 ist die zusammengesetzte Übersichtsaufnahme entlang des gesamten Risses zu sehen. Hier fällt auf, dass die Rissoberflächen relativ glatt sind und ein Helligkeitsgradient hin zu der Rissoberfläche vorhanden ist. Dieser Helligkeitsgradient bestätigt die bisherige Beobachtung, dass im Bereich des Risses vor dem eigentlichen Versagen die Schichten lokal eingeschnürt und damit dünner in Richtung des Elektronenstrahls werden. In der Nähe zum Substrat (links in Abbildung 4.52) ist diese Einschnürung weniger stark ausgeprägt. 


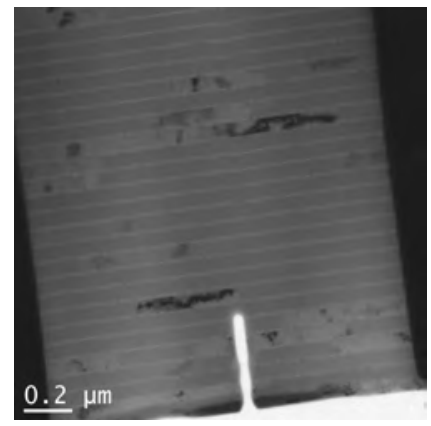

(a) BF Übersicht

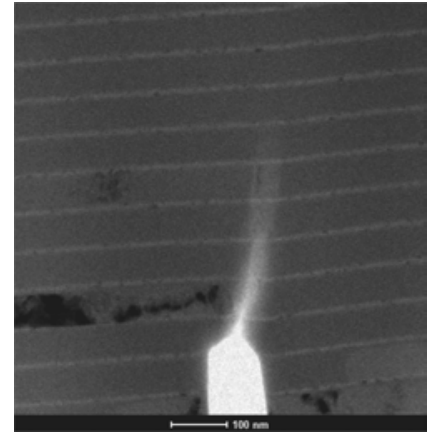

(d) Bild 165

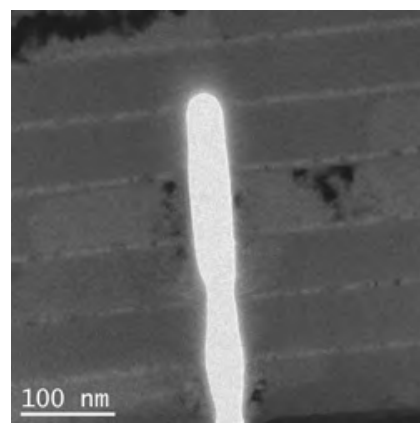

(b) BF Kerbe

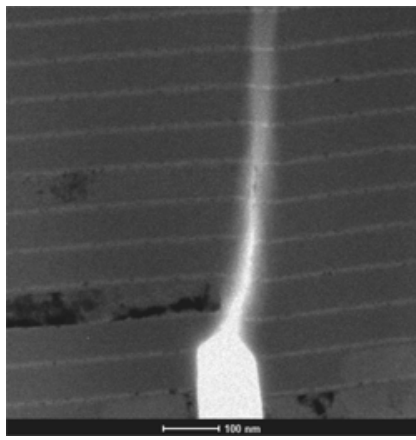

(e) Bild 186

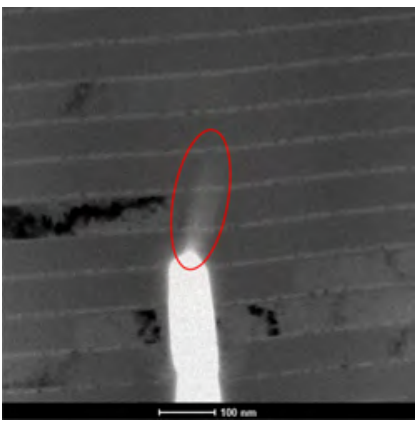

(c) Ende 1. Schritt

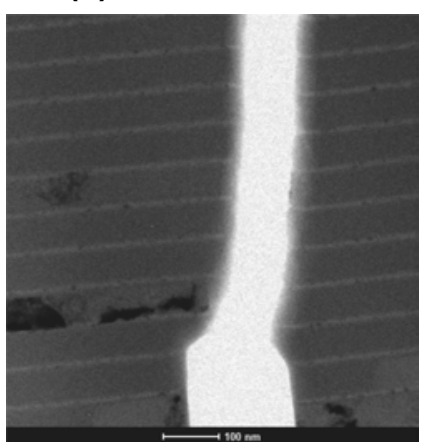

(f) Bild 188

Abbildung 4.51: Bruchversuch Ti10/ZrO 100 senkrecht zu den Grenzflächen Probe 1: Abbildungen (d) bis (f) zeigen Momentaufnahmen während des Risswachstums

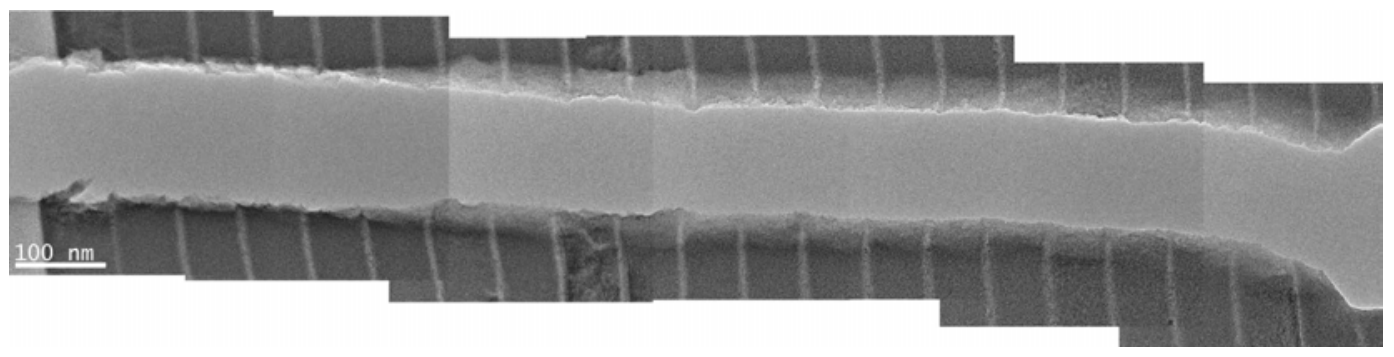

Abbildung 4.52: Bruchversuch Ti10/ZrO 100 senkrecht zu den Grenzflächen Probe 1 Übersicht Riss

\section{$\mathrm{Ti10} / \mathrm{ZrO}_{2} 100-$ Probe 2}

Als zweites Beispiel für das Bruchverhalten der Proben mit einer Ti-Schichtdicke von $d_{\mathrm{Ti}}=10 \mathrm{~nm}$ werden die gewonnenen Ergebnisse des Bruchversuchs mit der in Abbildung $4.53 \mathrm{a}$ dargestellten Probe im Folgenden beschrieben. Wie schon bei der Probe zuvor können in den $\mathrm{ZrO}_{2}$-Schichten verhältnismäßig viele kristalline Bereiche festgestellt werden. Die Spitze der Kerbe liegt, wie Abbildung 4.53b verdeutlicht, in der Mitte einer $\mathrm{ZrO}_{2}$-Schicht in direkter Umgebung von kristallinen Stellen im $\mathrm{ZrO}_{2}$. HRTEMAufnahmen (hier nicht gezeigt) bestätigen, dass das Material an der Spitze der Kerbe kristalline Strukturen aufweist.

Der eigentliche Bruchversuch dieser Probe ist in sechs einzelne Verformungsschritte unterteilt. Im ersten Schritt bewegen sich nur Biegekonturen in beiden Materialien. 


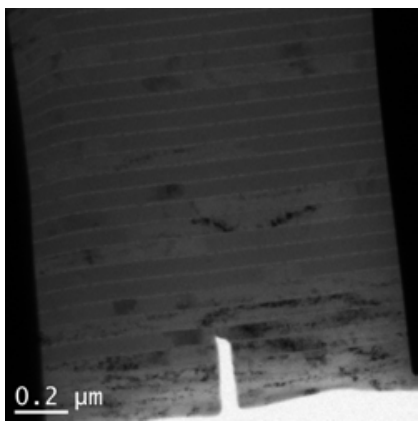

(a) BF Übersicht

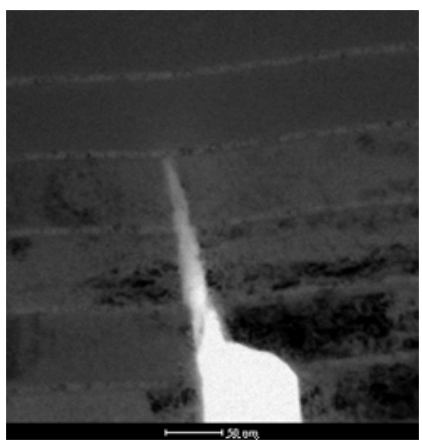

(d) Bild 464 3. Schritt

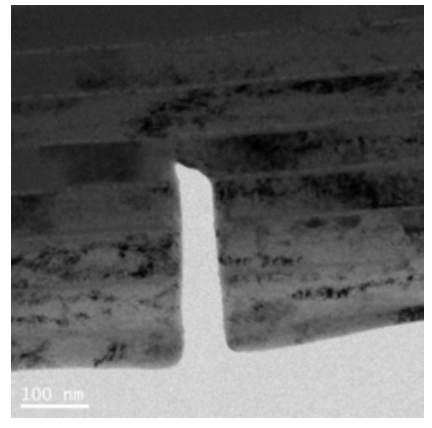

(b) BF Kerbe

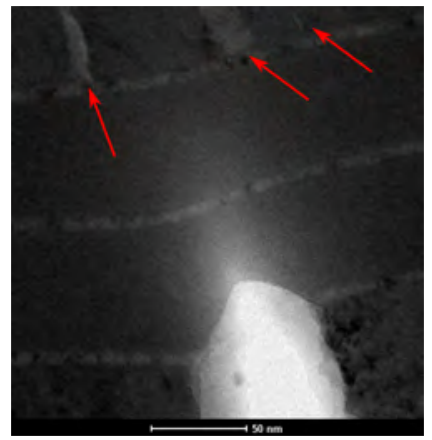

(e) Bild 450 6. Schritt

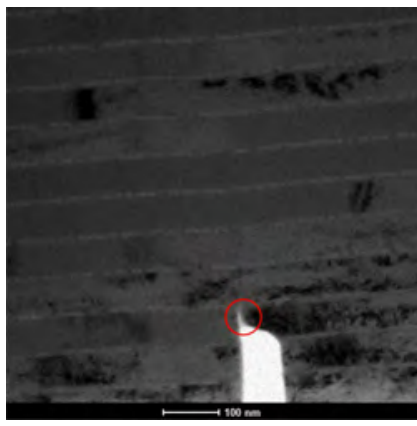

(c) Bild 486 2. Schritt

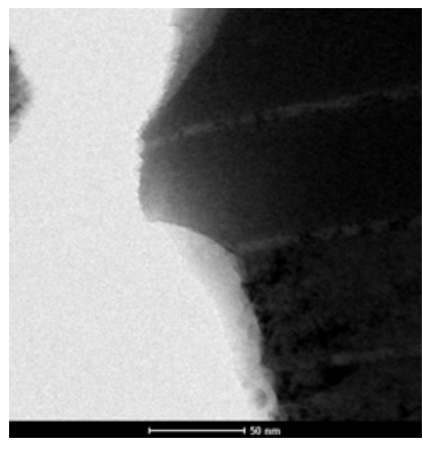

(f) Bild 460 6. Schritt

Abbildung 4.53: Bruchversuch $\mathrm{Ti10} / \mathrm{ZrO}_{2} 100$ senkrecht zu den Grenzflächen Probe 2

Biegekonturen sind auch im zweiten Schritt zu beobachten, zusätzlich wird ein Riss an der Spitze der Kerbe gebildet (vgl. Markierung in Abbildung 4.53c).

Das Wachstum und die Bewegung dieses Risses werden in den folgenden Verformungsschritten bei einer höheren Vergrößerung verfolgt. Während des dritten Schrittes wächst der Riss über die nächsten zwei Ti- und $\mathrm{ZrO}_{2}$-Schichten in Richtung des Si-Substrates (vgl. Abbildung 4.53d). Selbst bei höherer Vergrößerung kann eine Versetzungsaktivität nicht eindeutig beobachtet werden.

Im vierten und fünften Schritt wächst der Riss nicht weiter. Es kann jedoch eine Abrundung der Rissspitze beobachtet werden.

Erst im sechsten und letzten Schritt kann wieder eindeutig Verformung und Risswachstum erkannt werden. In Abbildungen 4.53e und 4.53f sind zwei Momentaufnahmen zum Ende der Bildserie gezeigt. In Abbildung 4.53e ist zu erkennen, dass die Rissspitze im Vergleich zu Abbildung 4.53d wesentlich abgerundeter ist. Weiterhin kann in der $\mathrm{ZrO}_{2}{ }^{-}$ Schicht, die zwei Schichten entfernt von der eigentlichen Rissspitze liegt, an mehreren Stellen eine Verformung beobachtet werden (Pfeile in Abbildung 4.53e). Im Bereich direkt vor der Rissspitze kann, wie bereits geschildert, die Einschnürung des Materials durch die Verformung festgestellt werden. Nur zehn Einzelbilder (Abbildung 4.53f) später versagt die Probe katastrophal. Der Riss wächst in Richtung der verformten Stellen im $\mathrm{ZrO}_{2}$ und von dort bis in das Si-Substrat.

In Abbildung 4.54 ist eine zusammengesetzte Übersichtsaufnahme des gesamten Ris- 


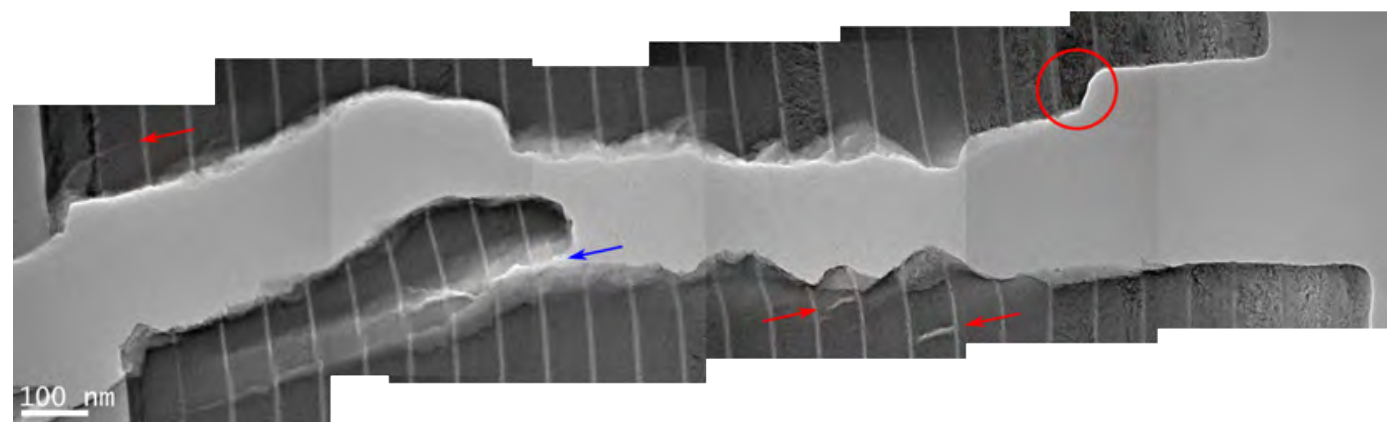

Abbildung 4.54: Bruchversuch Ti10/ZrO 100 senkrecht zu den Grenzflächen Probe 2 Übersicht Riss

ses zu sehen. Mit dem roten Kreis ist die ursprüngliche Position der Spitze der Kerbe markiert. In dieser Übersicht fällt auf, dass es an mehreren Stellen (rote Pfeile) verformte Bereiche in den $\mathrm{ZrO}_{2}$-Schichten gibt. Diese Verformungen liegen in der Nähe der Rissoberfläche, sind aber nicht Teil des eigentlichen Risses, der komplett durch die gesamte Multilage läuft.

Weiterhin ist zu beobachten, dass im mit dem blauen Pfeil markierten Bereich, der Riss abgelenkt und ein zweiter Riss (in Richtung des Pfeils) gebildet wird. Der Hauptriss, ausgehend von der Kerbe, wird an dieser Stelle um ca. $70 \mathrm{~nm}$ nach rechts (gesehen in Rissausbreitungsrichtung) abgelenkt, bevor er ohne weitere Ablenkung bis in das Substrat wächst. Der zweite Riss wächst parallel zum Hauptriss auch in Richtung Substrat, erreicht dieses aber nicht. Im Bereich vor der Rissspitze des zweiten Risses ist das Material bereits verformt, aber noch nicht gerissen.

Da sich der zweite Riss und die Ablenkung des Hauptrisses außerhalb des Bildausschnittes des sechsten Verformungsschrittes gebildet haben, kann über deren Entstehung keine gesicherte Aussage getroffen werden.

\section{Ti10/ZrO $2100-$ Probe 3}

In Abbildung 4.55a ist die Hellfeldübersichtsaufnahme der nächsten Probe dieser Schichtdicke gezeigt. Die Spitze der Kerbe liegt in diesem Fall wiederum in einer der $\mathrm{ZrO}_{2}$-Schichten, jedoch nur ca. $10 \mathrm{~nm}$ von der Grenzfläche zur nächsten Ti-Schicht entfernt. Zusätzlich weist der Bereich in der $\mathrm{ZrO}_{2}$-Schicht um die Spitze kristalline Strukturen auf.

Der Bruchversuch ist in vier Einzelschritte unterteilt. Im ersten Schritt bildet sich noch kein Riss. Lediglich die Öffnungsweite der Kerbe wird vergrößert und Biegekonturen in beiden Materialschichten können beobachtet werden. Im zweiten Schritt bildet sich ein erster kurzer Riss an der Kerbe (vgl. Markierung in Abbildung 4.55c). Dieser Riss reicht bis zum Ende des Verformungsschrittes bis in die angrenzende Ti-Schicht hinein. Im Bereich der auf die gerissene Ti-Schicht folgenden $\mathrm{ZrO}_{2}$-Schicht ist schon die Einschnürung des $\mathrm{ZrO}_{2}$ zu erkennen. Zu Beginn des dritten Verformungsschrittes bil- 


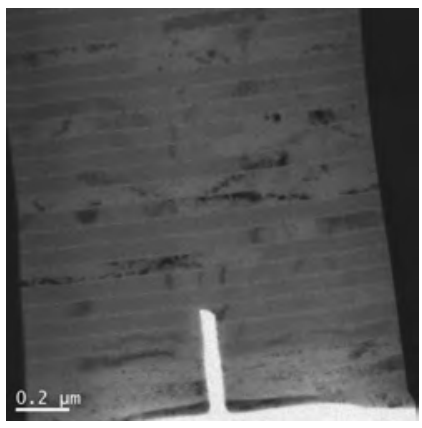

(a) BF Übersicht

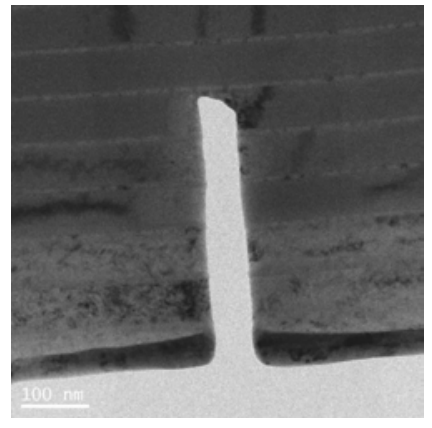

(b) BF Kerbe

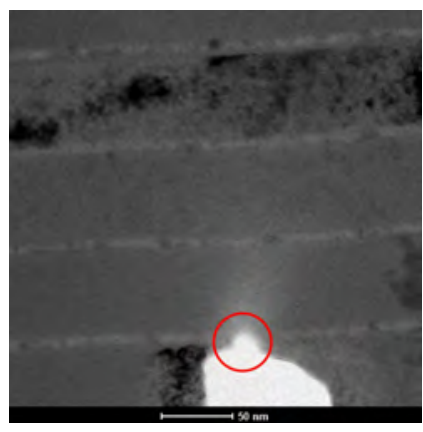

(c) Bild 453 2. Schritt

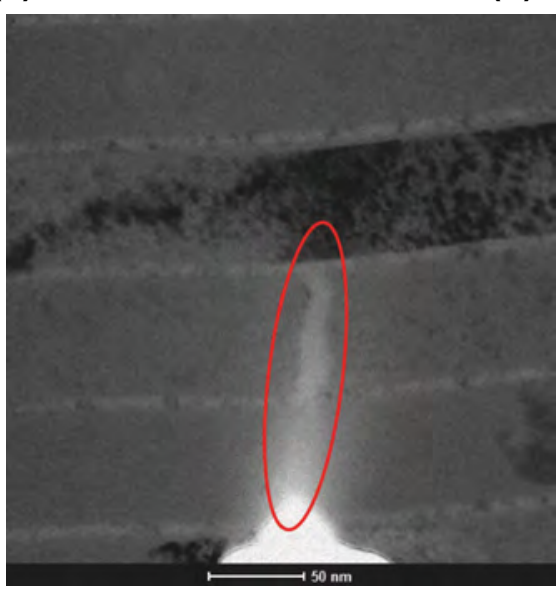

(d) Bild 490 3. Schritt

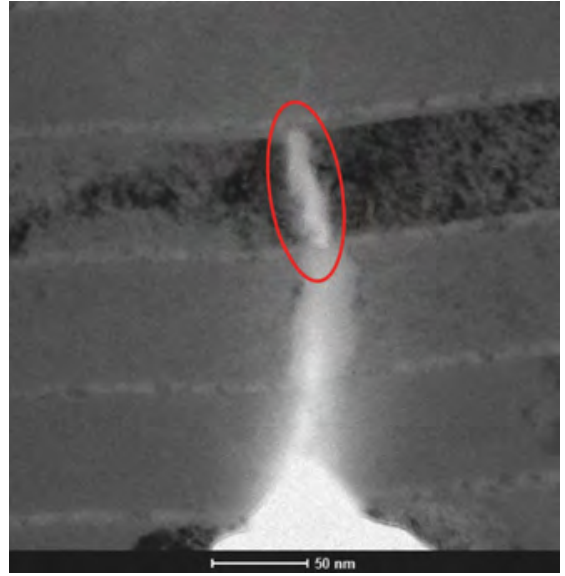

(e) Bild 345 4. Schritt

Abbildung 4.55: Bruchversuch $\mathrm{Ti10} / \mathrm{ZrO}_{2} 100$ senkrecht zu den Grenzflächen Probe 3

det sich zunächst eine verformte Stelle in der $\mathrm{ZrO}_{2}$-Schicht, die sich ursprünglich zwei $\mathrm{ZrO}_{2}$-Schichten von der Kerbe entfernt befindet. Diese Verformung bildet sich in einem Bereich helleren Kontrastes, jedoch ohne, dass die Schichten, die näher zur Kerbe liegen, gerissen sind. Im Verlauf des dritten Schrittes wächst der Riss ausgehend von der Kerbe hin zu der Verformung in der $\mathrm{ZrO}_{2}$-Schicht, sodass am Ende ein verformter Bereich bzw. Riss über die zwei Ti- und $\mathrm{ZrO}_{2}$-Schichten zu beobachten ist (vgl. Abbildung 4.55d).

Im letzten Schritt wächst der Riss weiter und der Bereich der Verformung in den $\mathrm{ZrO}_{2}$ Schichten verbreitert sich kontinuierlich. In der dunkel erscheinenden $\mathrm{ZrO}_{2}$-Schicht bildet sich innerhalb weniger Einzelbilder der Bildserie eine weitere verformte Stelle, die über die gesamte Schichtdicke reicht (vgl. Abbildung 4.55e). Wieder nur wenige Einzelbilder später versagt die Probe katastrophal und der Riss wächst bis in das Substrat. Die zusammengesetzte Aufnahme des Risses ist in Abbildung 4.56 gezeigt. Hier ist zu erkennen, dass im Bereich der verformten Stelle in der dunkleren $\mathrm{ZrO}_{2}$-Schicht (Markierung in Abbildung 4.55e der Riss abgelenkt und ein weiterer Riss gebildet wird. Durch den zweiten Riss wird ein Teil des Materials von der ursprünglichen Rissoberfläche abgelöst (vgl. Markierung in Abbildung 4.56). Nachdem der Hauptriss in der Nähe der Kerbe abgelenkt wird, wächst er im weiteren Durchgang durch die Lamelle 


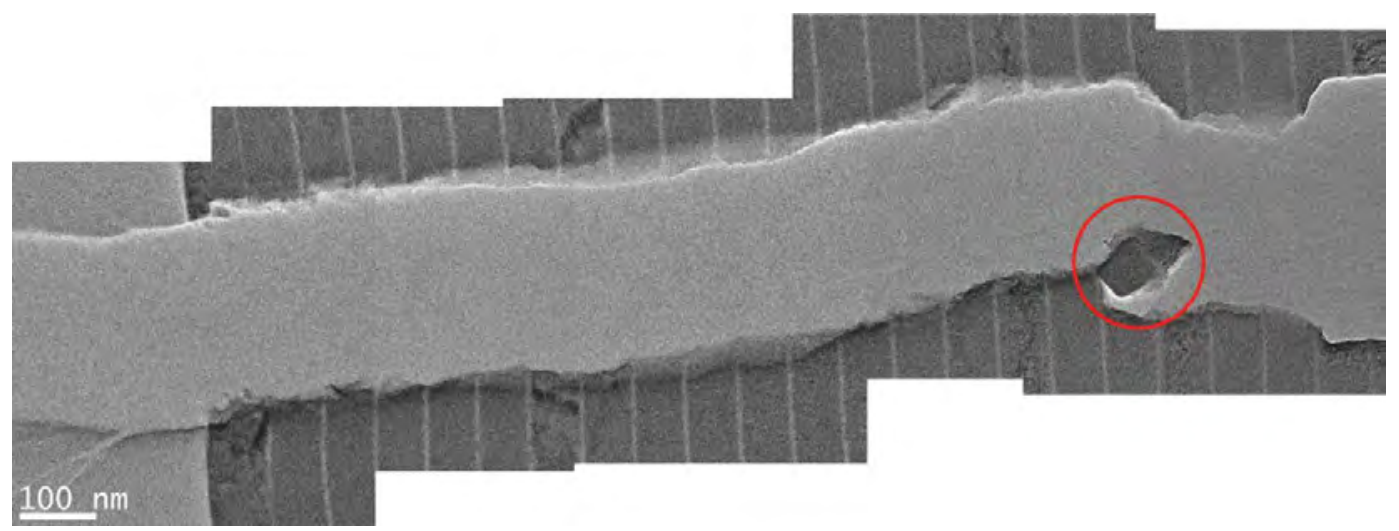

Abbildung 4.56: Bruchversuch Ti10/ZrO 2100 senkrecht zu den Grenzflächen Probe 3 Übersicht Riss

relativ gerade über alle Grenzflächen hinweg. Im Bereich der Mitte der Lamelle ist zu erkennen, dass hier ein Helligkeitsgradient an der Rissoberfläche vorhanden ist, sodass auch hier das Material, bevor es gerissen ist, eingeschnürt und damit dünner wird.

\section{$\mathrm{Ti10} / \mathrm{ZrO}_{2} 100-$ Probe 4}

Als letztes Beispiel werden die Ergebnisse des Bruchversuchs an der in Abbildung 4.57a dargestellten Lamelle dokumentiert. Die Spitze dieser Kerbe liegt direkt an der Grenzfläche zu einer benachbarten Ti-Schicht, wie in Abbildung 4.57b gezeigt. Dieser Bruchversuch wird in Dunkelfeldabbildungsbedingungen in nur einem Verformungsschritt durchgeführt. Bereits in diesem einen Schritt versagt die Lamelle katastrophal. In Abbildung 4.57c ist eine Dunkelfeldübersichtsaufnahme nach dem Bruch zu sehen. Der Riss hat sich an der Spitze der Kerbe gebildet und wächst mit einer deutlichen Tendenz hin zur rechten der beiden Si-Säulen.

Die Momentaufnahmen in Abbildungen 4.57d und 4.57e zeigen, dass sich ein Riss vor dem katastrophalen Versagen bildet, jedoch schon in der nächsten Aufnahme unkontrolliert durch die gesamte Lamelle gewachsen ist. In den wenigen Momentaufnahmen, in denen sich der Riss zuerst bildet und dann wächst, ist die Bewegung von Biegekonturen zu beobachten. An der Rissoberfläche der Schichten in der Nähe der Spitze der Kerbe ist der bereits bei anderen Lamellen beobachtete Helligkeitsgradient zu sehen. Das Material wird während des unkontrollierten Versagens erst eingeschnürt, bevor es letztlich reißt.

\section{Zusammenfassung $\mathrm{Ti} 10 / \mathrm{ZrO}_{2} 100$}

Die Bruchversuche der Probenserie mit der geringsten Ti-Schichtdicke versagen letztlich katastrophal bzw. unkontrollierbar. Wiederum werden die Risse an der Spitze der Kerbe gebildet, jedoch ist deren Ausbreitungsrichtung deutlich abgelenkt von der Richtung senkrecht zu den Grenzflächen. Zusätzlich zeigt sich ein Unterschied zu den Bruchver- 


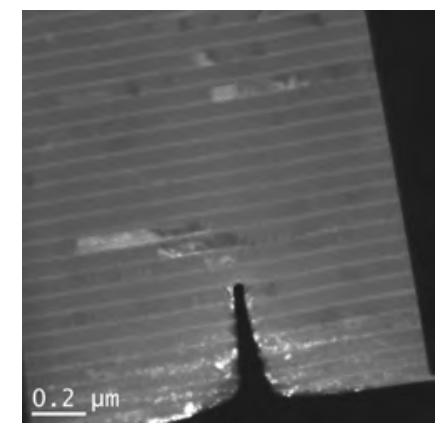

(a) DF Übersicht

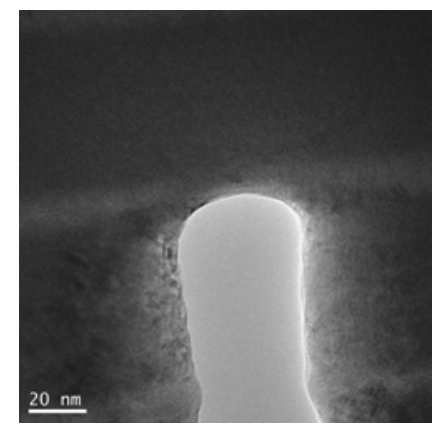

(b) BF Kerbe

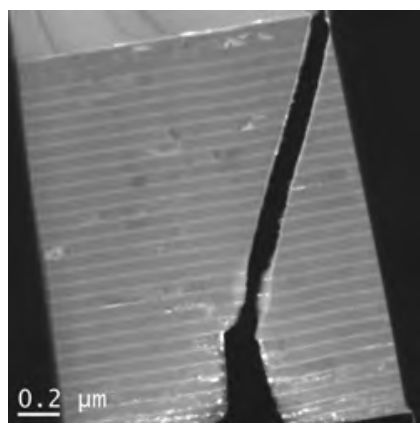

(c) DF Übersicht Riss

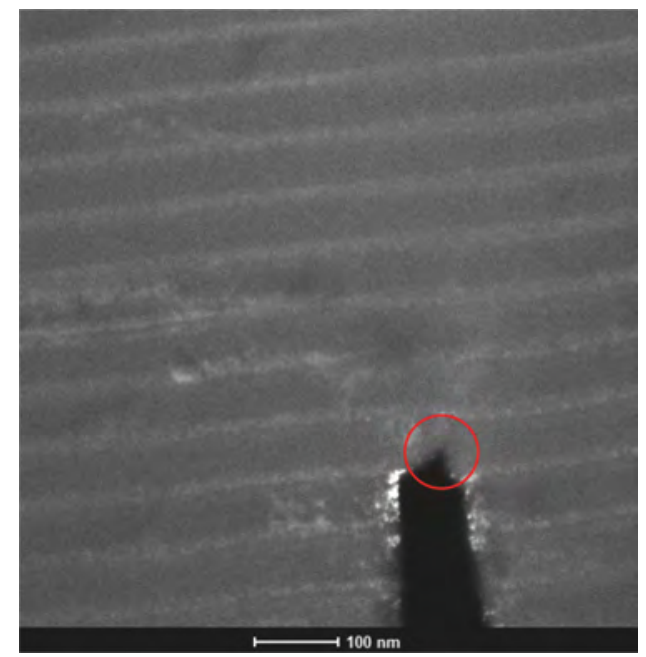

(d) Bild 142

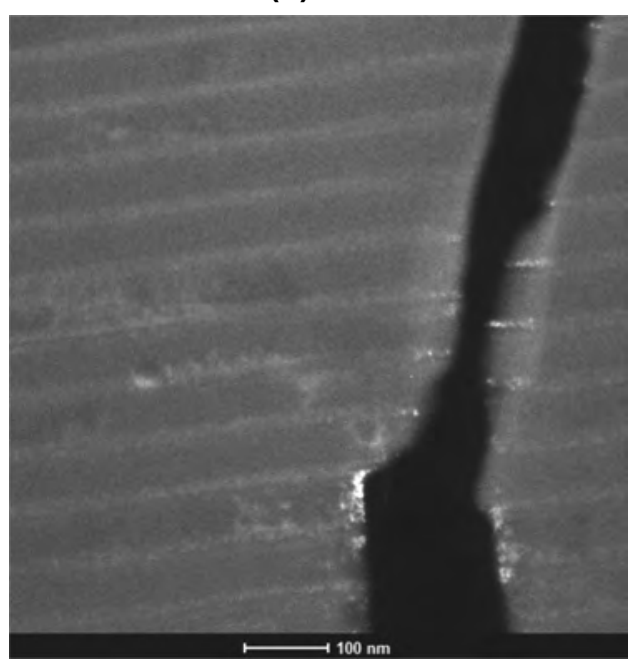

(e) Bild 143

Abbildung 4.57: Bruchversuch Ti10/ZrO 100 senkrecht zu den Grenzflächen Probe 4: Abbildung (d) und (e) zeigen die Lamelle vor und nach dem katastrophalen Versagen.

suchen der größeren Ti-Schichtdicken, da es keine Ausbildung von Rissbrücken gibt. Die Rissoberflächen sind aufgrund des katastrophalen Versagens sehr gerade und glatt. 


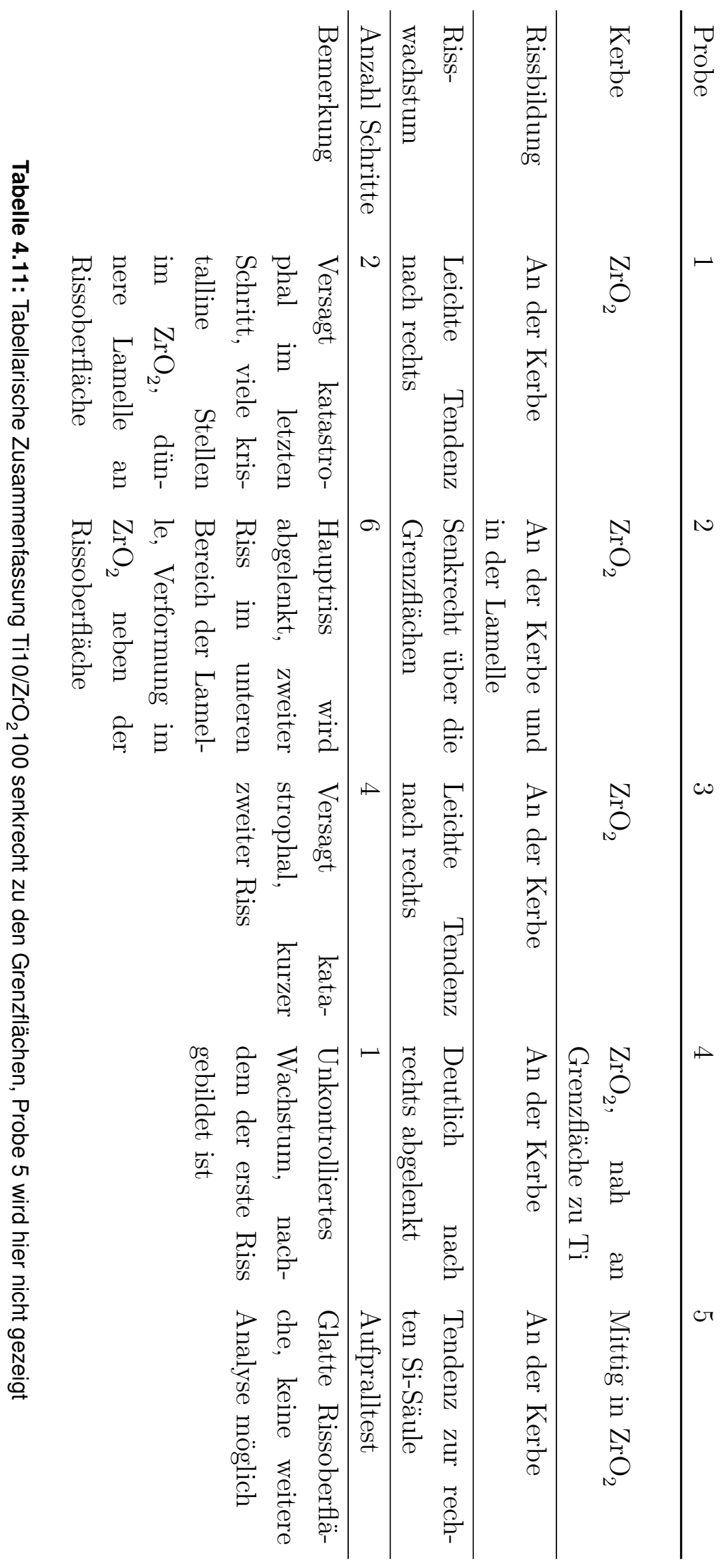




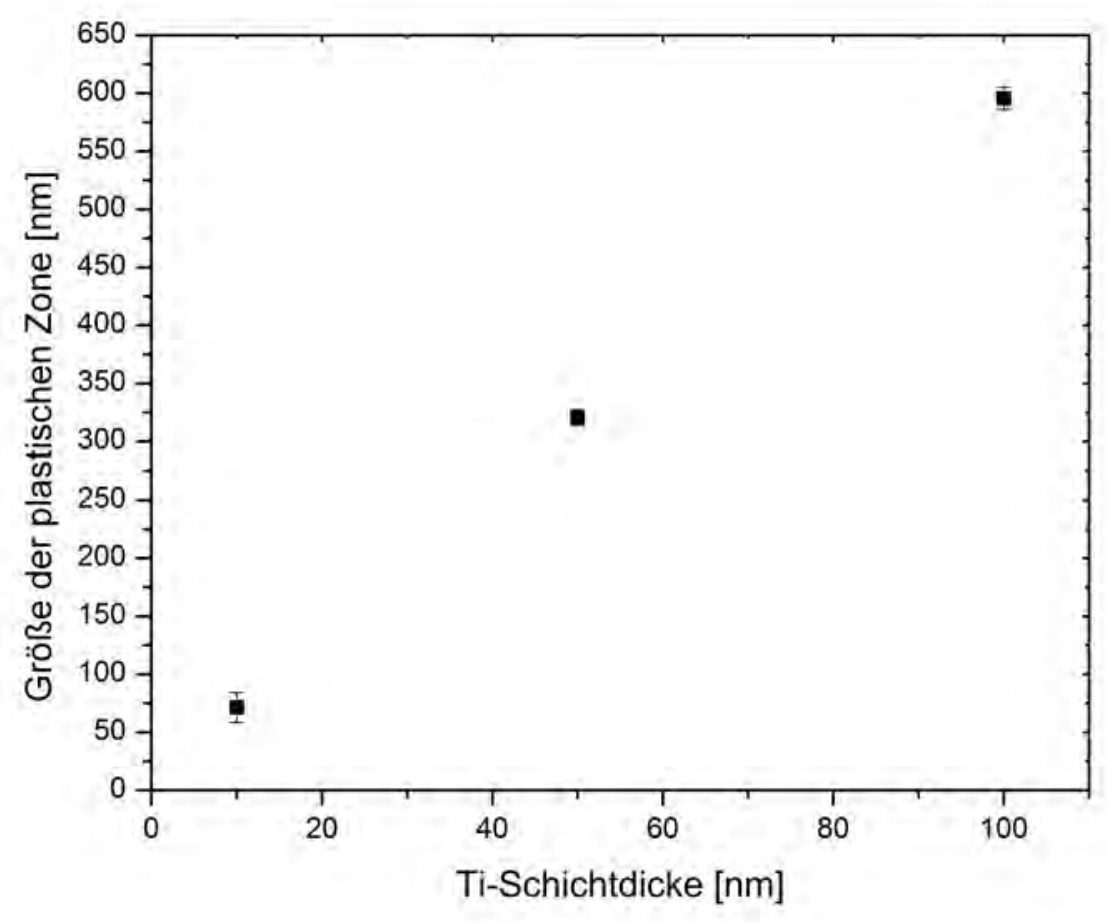

Abbildung 4.58: Die Größe der plastischen Zone in Abhängigkeit der Ti-Schichtdicke für die Bruchversuche senkrecht zu den Grenzflächen.

\subsubsection{Plastische Zone}

Wie bereits für die Bruchversuche entlang der Grenzflächen, werden auch für die Bruchversuche senkrecht zu den Grenzflächen in allen geeigneten Bildserien die Positionen der schnellen Kontrastwechsel als Maß für die Größe der plastischen Zone genutzt. Alle dargestellten Werte sind gewichtete Mittelwerte verschiedener Lamellen und Bildserien einer Schichtdickenkombination. In Abbildung 4.58 ist die Größe der plastischen Zone in Abhängigkeit der Schichtdicke aufgetragen.

\subsubsection{Energiefreisetzungsrate}

Die Analyse der kritischen Energiefreisetzungsrate erfolgt für alle geeigneten Bildserien der gezeigten Bruchversuche entsprechend der in Abschnitt 3.4.1 geschilderten Methodik. Im Gegensatz zu der Auswertung der Energiefreisetzungsrate für die Bruchversuche entlang der Grenzflächen werden hier jeweils die Kompositwerte der Fließspannung sowie des Elastizitätsmoduls der jeweiligen Schichtdickenkombination verwendet (vgl. Tabelle 3.2. In Abbildung 4.59 sind die so erhaltenen Werte für $J_{I Q}$ und $K_{I Q}$ gegen die Ti-Schichtdicke aufgetragen.

In Abbildung 4.60 sind exemplarisch die Risswiderstandskurven für jedes Schichtdi- 


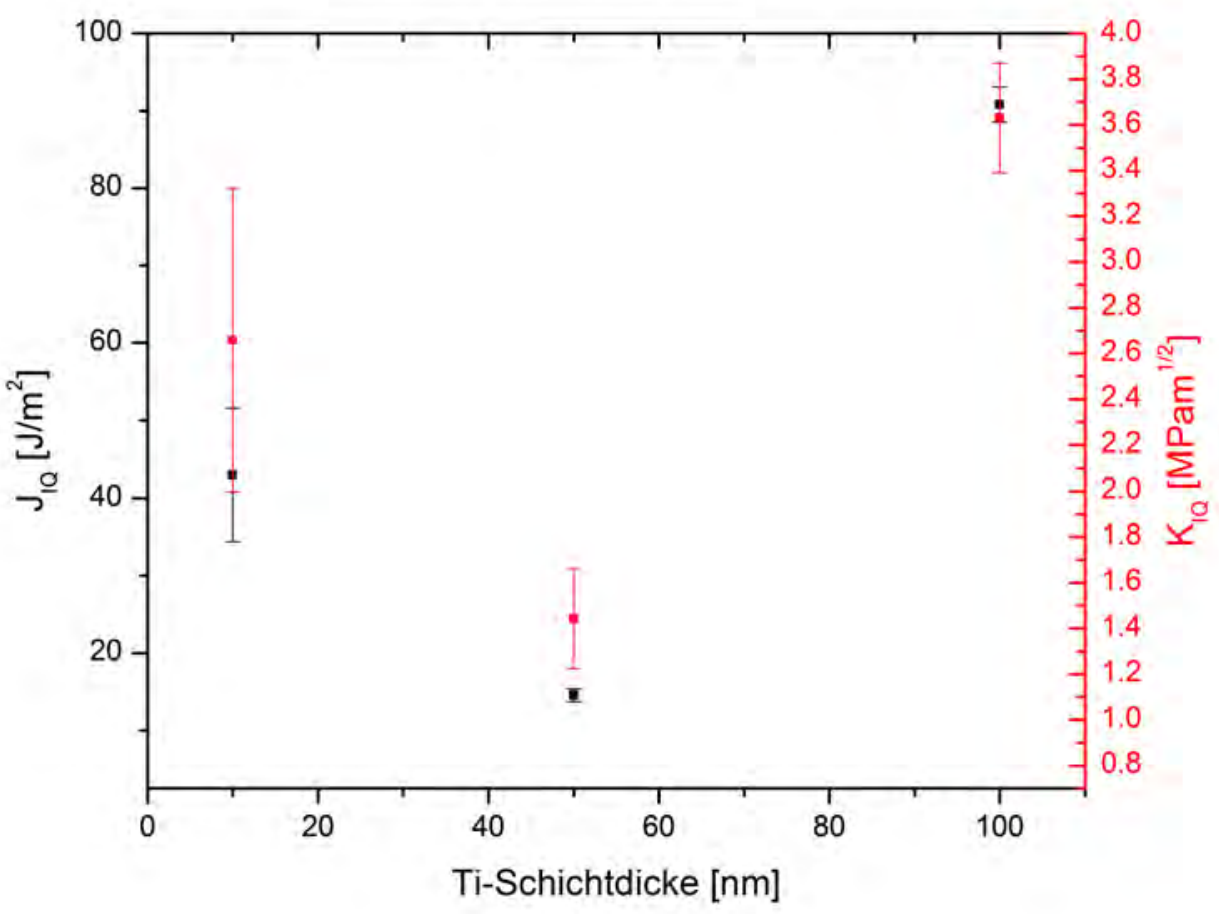

Abbildung 4.59: Kritische Energiefreisetzungsrate und Bruchzähigkeit in Abhängigkeit der TiSchichtdicke für die Bruchversuche senkrecht zu den Grenzflächen.

ckenverhältnis gezeigt. 


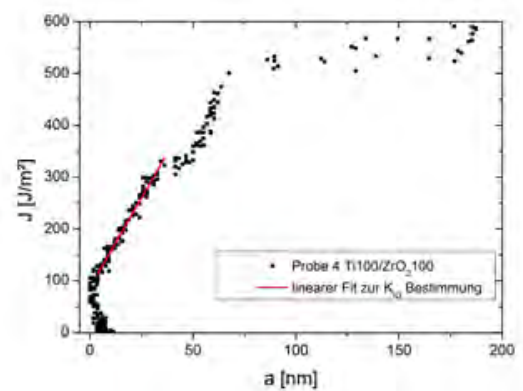

(a) $\mathrm{Ti} 100 / \mathrm{ZrO}_{2} 100$ Probe 4

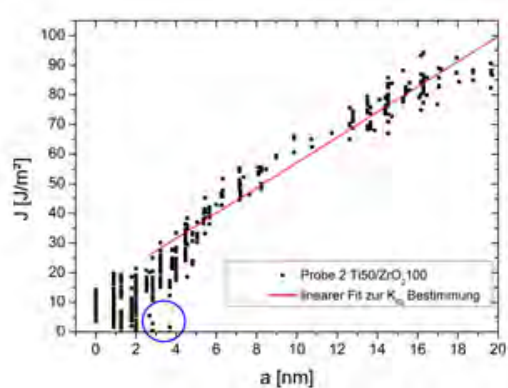

(b) $\mathrm{Ti} 50 / \mathrm{ZrO}_{2} 100$ Probe 2

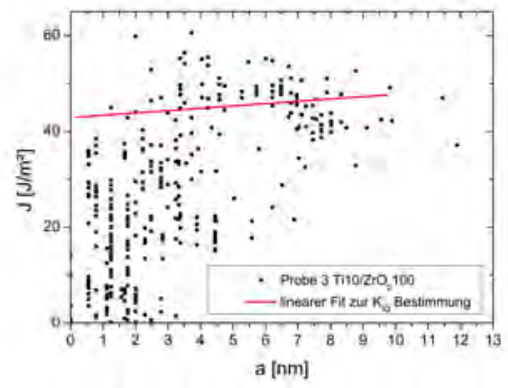

(c) $\mathrm{Ti} 10 / \mathrm{ZrO}_{2} 100$ Probe 3

Abbildung 4.60: Risswiderstandskurven Bruchversuche senkrecht zu den Grenzflächen 


\subsection{Einfluss einer Gasatmosphäre auf das Bruchverhal- ten}

In den nachfolgenden Abschnitten werden die Bruchversuche in einer Gasatmosphäre beschrieben. Es werden Experimente in einer Wasserstoff- und einer WasserAtmosphäre durchgeführt. Der Druck im Probenraum des verwendeten ETEM ist für beide Gase etwa 6 mbar. Sowohl für die Wasserstoff- als auch für die WasserAtmosphäre gilt, dass ausgehend vom Vakuumlevel des Mikroskops der Druck kontinuierlich erhöht wird und während der Experimente ein Gasfluss durch den Probenraum den Druck konstant hält. Der Gasfluss ist für beide Gase auf $10 \mathrm{~cm}^{3} \mathrm{~min}^{-1}$ limitiert. Alle Proben werden vorab ohne Gasatmosphäre und auch nach dem Entfernen des Gases charakterisiert. Um etwaige chemische Veränderungen der Proben festzustellen, werden EELS-Spektren aufgenommen. Aus dem Vergleich der Spektren vor dem Einlassen des Gases mit den Spektren in der Gasatmosphäre und nach dem Bruchversuch (ohne Gas) können Indizien für Veränderungen in der Probe gewonnen werden. Die EELS-Daten werden abschließend zusammenfassend dargestellt.

\subsubsection{Wasserstoff-Atmosphäre}

Ti100/ZrO 2100 entlang der Grenzflächen

Abbildung 4.61a zeigt die Hellfeldübersichtsaufnahme der Ti100/ $\mathrm{ZrO}_{2}$ 100-Lamelle, die auf den Einfluss eines Wasserstoffdrucks auf ihr Bruchverhalten hin untersucht wird. Die Spitze der Kerbe liegt, wie die HRTEM-Aufnahme in Abbildung 4.61b zeigt, in einer der $\mathrm{ZrO}_{2}$-Schichten. Die bereits bekannte Kristallisation des $\mathrm{ZrO}_{2}$ durch den Ionenstrahl kann auch hier beobachtet werden.

Der Bruchversuch ist insgesamt in drei Verformungsschritte unterteilt, wobei im ersten der Schritte neben der Bewegung der Biegekonturen keine weiteren Veränderungen der Lamelle festgestellt werden können.

Erst im zweiten Verformungsschritt kann die Aktivität von Versetzungen in den TiSchichten deutlich erkannt werden, zusätzlich beginnt ein Riss von der Kerbe aus in der $\mathrm{ZrO}_{2}$-Schicht zu wachsen. Dieser Riss wächst im letzten Schritt weiter, bevor es in der dritten $\mathrm{ZrO}_{2}$-Schicht rechts von der Kerbe (außerhalb des Bildausschnittes in der

Bildserie) zu einem unkontrollierten Risswachstum kommt. In Abbildung 4.61c ist das letzte Bild der Bildserie des dritten Schrittes gezeigt, bevor die $\mathrm{ZrO}_{2}$-Schicht katastrophal versagt.

In Abbildung 4.61d ist eine zusammengesetzte Hellfeldübersichtsaufnahme des Risses in der $\mathrm{ZrO}_{2}$-Schicht gezeigt. Der Riss liegt über eine Länge von ca. 8,2 $\mu \mathrm{m}$ durchgehend in der $\mathrm{ZrO}_{2}$-Schicht, in der er auch gebildet wurde. Im Bereich, in dem der unkontrol- 


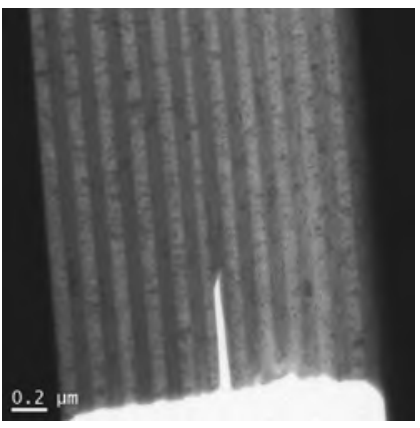

(a) BF Übersicht

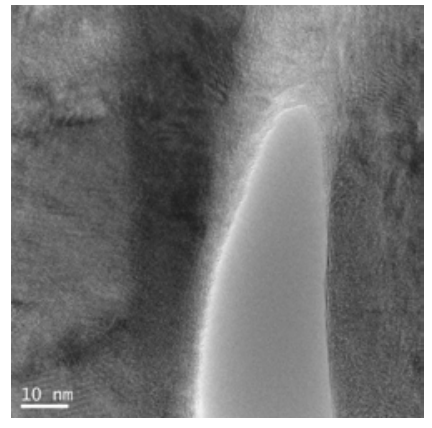

(b) HRTEM Kerbe

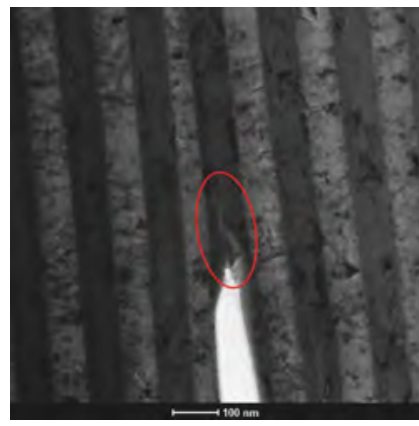

(c) Bild 3003 .

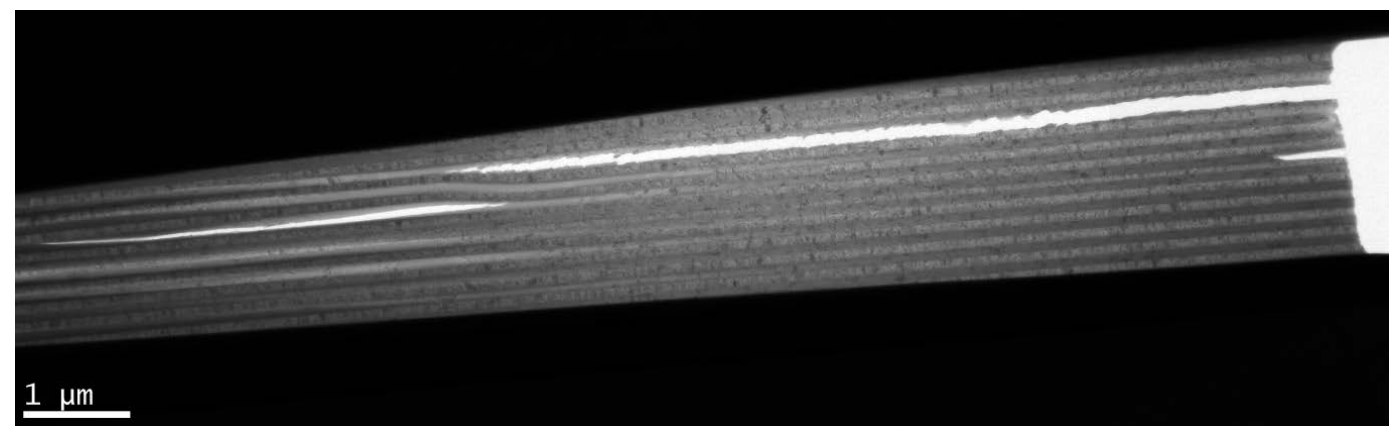

(d) Übersicht Riss nach Bruch

Abbildung 4.61: Bruchversuch Ti100/ZrO 100 entlang der Grenzflächen Wasserstoff-Atmosphäre

\begin{tabular}{cccc} 
Lamelle & $r_{p}[\mathrm{~nm}]$ & $J_{I Q}\left[\mathrm{~J} \mathrm{~m}^{-2}\right]$ & $K_{I Q}\left[\mathrm{MPa} \mathrm{m}^{1 / 2}\right]$ \\
\hline \hline 1 & $627(32)$ & $3,751(251)$ & $0,743(130)$
\end{tabular}

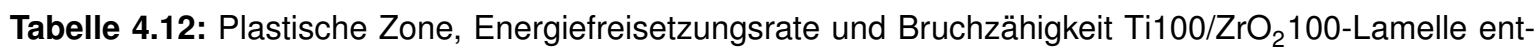
lang der Grenzflächen in $\mathrm{H}_{2}$

liert gewachsene Riss endet, ist in einer weiteren $\mathrm{ZrO}_{2}$-Schicht ein neuer Riss zu sehen. Dieser Riss ist in der Lamelle gebildet worden, ohne dass es eine Kerbe gibt. Auch er liegt komplett in der $\mathrm{ZrO}_{2}$-Schicht, in der er erzeugt wurde.

In dem Bereich des zuletzt gebildeten Risses zeigen die $\mathrm{ZrO}_{2}$-Schichten einen Kontrastwechsel hin zu deutlich hellerem Kontrast. Diese Kontraständerung wird in Verbindung mit der außergewöhnlichen Verformung in diesem Bereich gebracht.

Der entstandene Riss in einer zweiten, in der Wasserstoff-Atmosphäre getesteten Lamelle, ist in Abbildung 4.62 gezeigt. Ausgehend von der Kerbe (rechts in Abbildung 4.62 wird der Riss in der $\mathrm{ZrO}_{2}$-Schicht mit der Kerbe nukleiert und wächst unkontrolliert bis zu dem mit dem roten Pfeil markierten Bereich. Anschließend geht der Riss in die benachbarte Ti-Schicht über und kreuzt zwei weitere $\mathrm{ZrO}_{2}$-Schichten, bevor er kontinuierlich in einer Ti-Schicht bis zum Ende des Bruchversuchs weiter wächst. In Tabelle 4.12 sind die Größe der plastischen Zone und die kritischen Werte $J_{I Q}$ und $K_{I Q}$ dargestellt. 


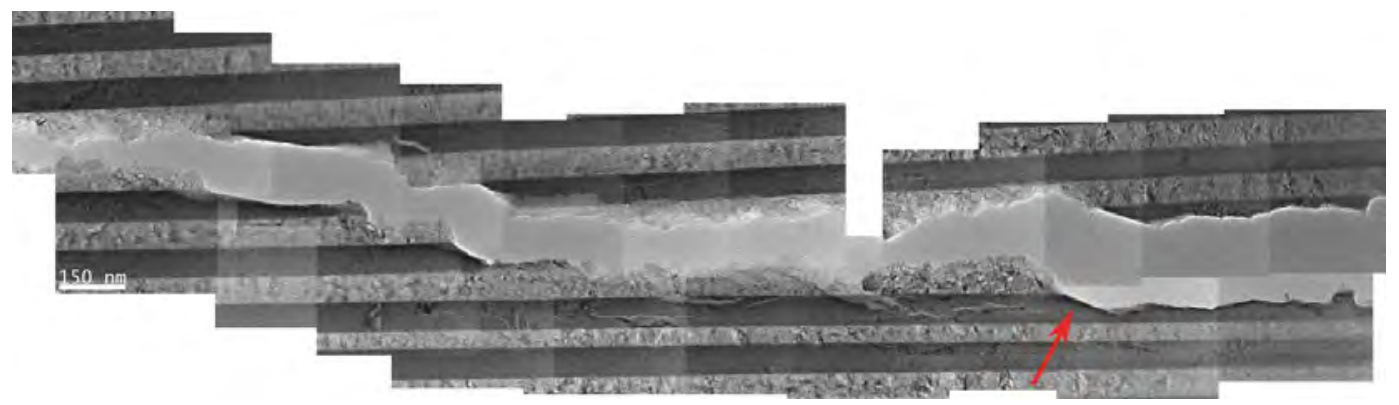

Abbildung 4.62: Bruchversuch Ti100/ZrO 100 entlang der Grenzflächen Wasserstoff-Atmosphäre Riss Lamelle 2

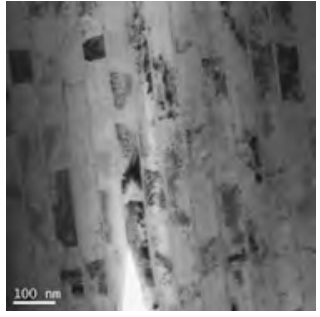

(a) BF Übersicht

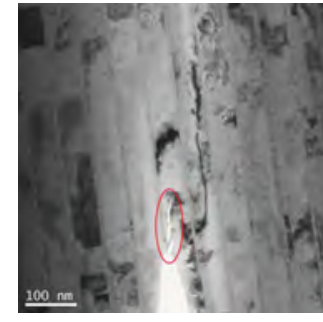

(b) 2. Schritt

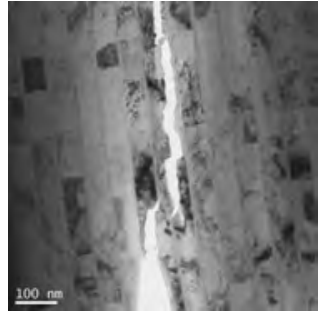

(c) 3. Schritt

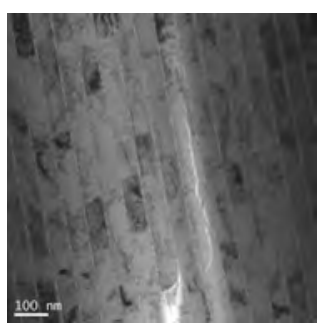

(d) 5 . Schritt

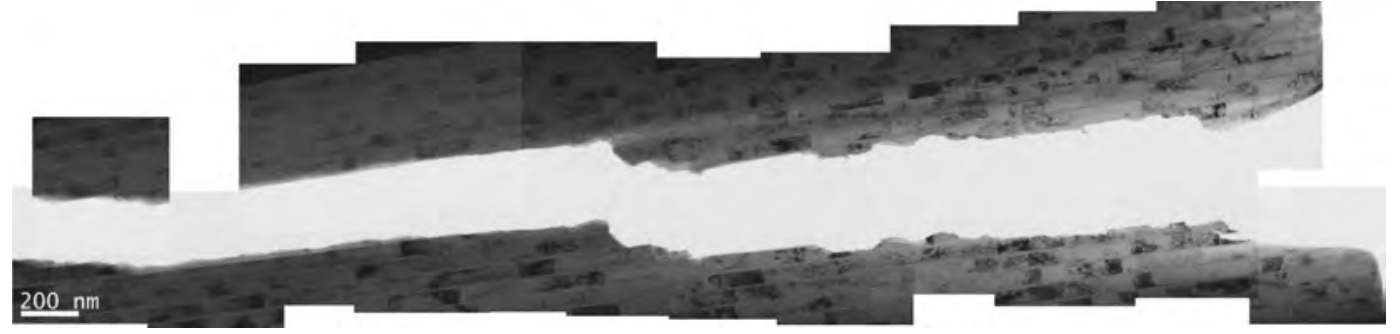

(e) Übersicht Riss nach Bruch

Abbildung 4.63: Bruchversuch Ti10/ZrO 100 entlang der Grenzflächen Wasserstoff-Atmosphäre

\section{Ti10/ZrO 2100 entlang der Grenzflächen}

Die in Abbildung 4.63a dargestellte Lamelle der kleinsten Ti-Schichtdicke wird ebenfalls in einer Wasserstoff-Atmosphäre getestet. Der Bruchversuch ist in diesem Fall in sechs einzelne Verformungsschritte/Bildserien unterteilt. Während des zweiten Verformungsschrittes bildet sich schlagartig ein Riss in der $\mathrm{ZrO}_{2}$-Schicht, wie in Abbildung $4.63 \mathrm{~b}$ markiert, obwohl die Spitze der Kerbe in beiden Schichten liegt. Neben diesem Riss bewegen sich in den ersten beiden Verformungsschritten Biegekonturen, die aufgrund der Kristallisation des $\mathrm{ZrO}_{2}$ in diesen Schichten beobachtet werden können.

Im dritten Schritt ergibt sich die Beobachtung, dass der vorhandene Riss wächst und in Richtung der Grenzfläche zu der auf der rechten Seite benachbarten Ti-Schicht abgelenkt wird. Bevor dieser Riss jedoch die Grenzfläche erreicht, ist eine erneute Rissbildung in der benachbarten $\mathrm{ZrO}_{2}$-Schicht zu erkennen. Dieser neue Riss wird mittig in der $\mathrm{ZrO}_{2}$-Schicht nukleiert, ohne dass diese Schicht vorab gerissen oder signifikant 


\begin{tabular}{cccc} 
Lamelle & $r_{p}[\mathrm{~nm}]$ & $J_{I Q}\left[\mathrm{~J} \mathrm{~m}^{-2}\right]$ & $K_{I Q}\left[\mathrm{MPa} \mathrm{m}^{1 / 2}\right]$ \\
\hline \hline 1 & - & $31,706(6341)$ & $2,348(1126)$
\end{tabular}

Tabelle 4.13: Plastische Zone, Energiefreisetzungsrate und Bruchzähigkeit $\mathrm{Ti}_{10} / \mathrm{ZrO}_{2} 100$-Lamelle entlang der Grenzflächen in $\mathrm{H}_{2}$

verformt ist. Der neue Riss wächst im Verlauf des dritten Verformungsschrittes in der Schicht, in der er erzeugt wurde. Das Risswachstum erfolgt nicht kontinuierlich, sondern in mehreren Sprüngen. In Abbildung 4.63c sind die beiden Risse am Ende des dritten Schrittes zu sehen. Die Ti-Schicht, die zwischen den beiden gerissenen $\mathrm{ZrO}_{2^{-}}$ Schichten liegt, ist noch intakt.

Im vierten Schritt wird die Rissspitze des zweiten Risses genauer beobachtet. Der zweite Riss wächst in diesem Verformungsschritt weiter in der $\mathrm{ZrO}_{2}$-Schicht, bewegt sich jedoch in Richtung der Grenzfläche auf der rechten Seite. Wie schon im dritten Schritt, bildet sich in der nächsten $\mathrm{ZrO}_{2}$-Schicht rechts von der bereits gerissenen $\mathrm{ZrO}_{2}$-Schicht ein nächster neuer Riss. Dieser wird wieder mittig in der $\mathrm{ZrO}_{2}$-Schicht erzeugt. Der Riss verläuft hier für ca. $400 \mathrm{~nm}$ entlang der Grenzfläche zwischen der Ti- und der $\mathrm{ZrO}_{2}$-Schicht (EELS-Daten werden hier nicht gezeigt.)

Im fünften Schritt breitet sich der Riss weiter entlang der Grenzfläche aus und verformt zusätzlich noch die benachbarte $\mathrm{ZrO}_{2}$-Schicht, die dadurch zu reißen beginnt, aber noch nicht komplett versagt (vgl. Abbildung 4.63d).

Im sechsten Schritt versagt die Probe letztlich katastrophal. In Abbildung 4.63e ist der gesamte Riss am Ende des Bruchversuchs gezeigt. Es ist zu erkennen, dass während des unkontrollierten Wachstums der Riss mehrere $\mathrm{ZrO}_{2}$ - und Ti-Schichten gekreuzt hat. In Tabelle 4.13 sind die Größe der plastischen Zone und die kritischen Werte $J_{I Q}$ und $K_{I Q}$ gelistet. Eine Größe der plastischen Zone kann für die Lamelle nicht abgeschätzt werden, da in den dünnen Ti-Schichten keine Versetzungsaktivität beobachtet werden konnte.

\section{$\mathrm{Ti50} / \mathrm{ZrO}_{2} 100$ senkrecht zu den Grenzflächen}

Hier wird eine Lamelle mit einer $50 \mathrm{~nm}$ dicken Ti-Schicht unter dem Einfluss einer Wasserstoff-Atmosphäre in der Geometrie senkrecht zu den Grenzflächen auf ihr Bruchverhalten hin getestet. In Abbildung 4.64a ist eine Hellfeldübersichtsaufnahme dieser Lamelle zu sehen. Die Spitze der Kerbe endet in einer Ti-Schicht, jedoch sehr nah an der Grenzfläche zu einer $\mathrm{ZrO}_{2}$-Schicht (vgl. Abbildung 4.64b). Der Bruchversuch beinhaltet in diesem Fall sechs Verformungsschritte. Im zweiten Schritt bildet sich ausgehend von der Kerbe ein Riss, wie in Abbildung 4.64c zu sehen ist. Wie auch in den Bruchversuchen ohne Gaseinfluss reißt zuerst die weiter entfernte $\mathrm{ZrO}_{2}$-Schicht, bevor die Ti-Schicht, in der die Spitze der Kerbe endet, reißt. Auch hier überbrücken die 


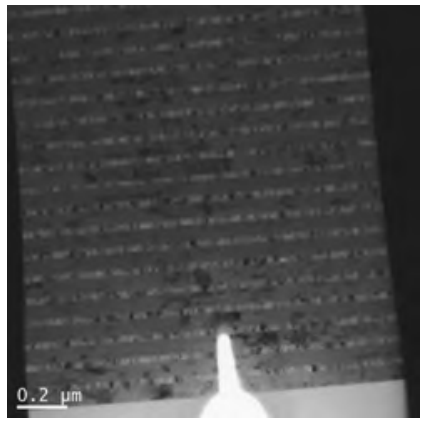

(a) BF Übersicht

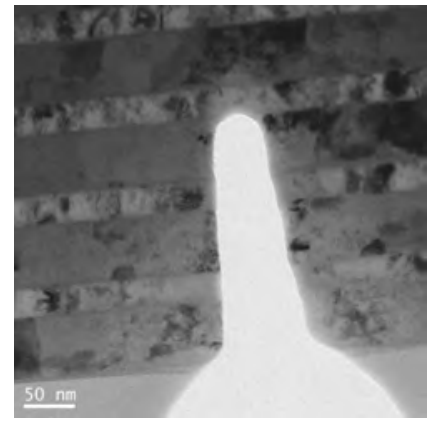

(b) BF Kerbe

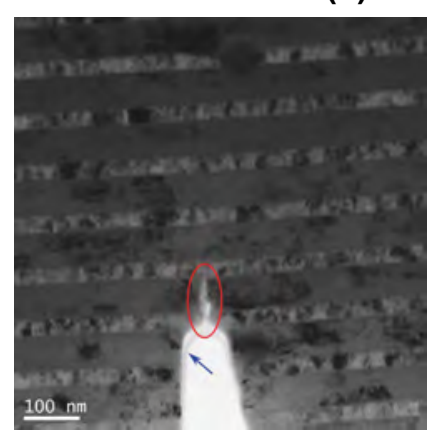

(c) BF nach 2. Schritt

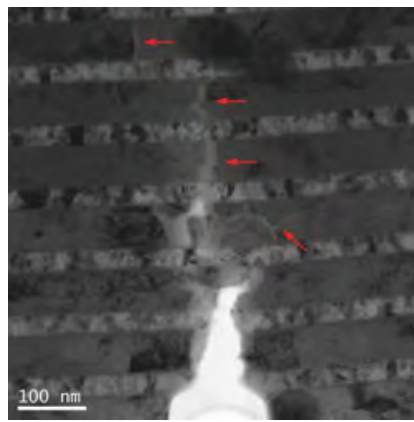

(d) BF nach 5. Schritt

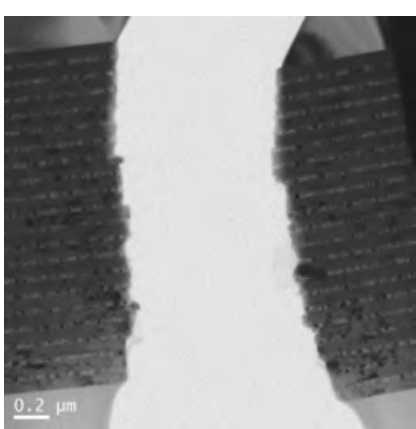

(e) BF Bruch

Abbildung 4.64: Bruchversuch Ti50/ZrO 100 senkrecht zu den Grenzflächen Wasserstoff-Atmosphäre

Ti-Schichten den Riss in die nächste $\mathrm{ZrO}_{2}$-Schicht.

Der helle Saum im Bereich der Kerbe (blauer Pfeil in Abbildung 4.64c) ist eine Schicht, die im Zusammenhang mit der Wasserstoffatmosphäre und dem Elektronenstrahl an der Oberfläche der Kerbe/Lamelle abgelagert wird.

Im dritten, vierten und fünften Schritt wächst der Riss weiter und es bildet sich eine komplexe Rissstruktur vor der eigentlichen Rissspitze aus. In Abbildung 4.64d ist der Riss am Ende des fünften Schrittes gezeigt. Auch hier ist zu beobachten, wie die $\mathrm{ZrO}_{2}$-Schichten bereits verformt/gerissen sind (rote Pfeile), obwohl die benachbarten Ti-Schichten noch intakt sind. Der Riss ist in dem Bereich der zweiten $\mathrm{ZrO}_{2}$-Schicht nach der Spitze der Kerbe leicht nach rechts hin abgelenkt. Anhand der dunklen Kontraste in den $\mathrm{ZrO}_{2}$-Schichten kann festgestellt werden, dass die $\mathrm{ZrO}_{2}$-Schichten kristallin sind. In allen Verformungsschritten können Versetzungsaktivitäten als Maß für die Größe der plastischen Zone analysiert werden.

Im letzten Schritt versagt die Probe katastrophal, sodass die Konfiguration, wie in Abbildung $4.64 \mathrm{e}$ gezeigt, nach dem Bruchversuch übrig bleibt. Der Riss hat sich unkontrolliert, aber relativ gerade über alle Grenzflächen hin bis in das Substrat ausgebreitet. In Tabelle 4.14 sind die Werte der quantitativen Auswertung dieser Lamelle dargestellt. 


\begin{tabular}{cccc} 
Lamelle & $r_{p}[\mathrm{~nm}]$ & $J_{I Q}\left[\mathrm{~J} \mathrm{~m}^{-2}\right]$ & $K_{I Q}\left[\mathrm{MPa} \mathrm{m}^{1 / 2}\right]$ \\
\hline \hline 1 & $260(39)$ & $77,956(716)$ & $3,337(81)$
\end{tabular}

Tabelle 4.14: Plastische Zone, Energiefreisetzungsrate und Bruchzähigkeit Ti50/ZrO 2 100-Lamelle senkrecht zu den Grenzflächen in $\mathrm{H}_{2}$

\subsubsection{Wasser-Atmosphäre}

Unter einer Wasser-Atmosphäre werden zwei Ti100/ $\mathrm{ZrO}_{2} 100-P r o b e n$ in der Geometrie entlang der Grenzflächen untersucht. In Abbildung 4.65a ist die Hellfeldaufnahme einer Lamelle ohne Gasatmosphäre und vor dem Bruchversuch gezeigt. Die Spitze der Kerbe liegt sowohl in einer Ti- als auch in einer $\mathrm{ZrO}_{2}$-Schicht (vgl. Abbildung 4.65b). Zusätzlich ist ein kleiner Riss an der Spitze der Kerbe zu erkennen, der in Richtung der benachbarten Ti-Schicht zeigt. Der Bruchversuch ist in sieben Verformungsschritte unterteilt. Im ersten und zweiten Schritt zeigen sich Bewegungen von Biegekonturen in den Ti-Schichten, wobei speziell zum Ende des zweiten Schrittes mit der Öffnung der Kerbe zusätzlich die Aktivität von Versetzungen in der Nähe der Spitze der Kerbe beobachtet werden kann.

Im dritten Schritt wird zuerst ein signifikantes Risswachstum beobachtet. Ausgehend von dem kleinen Riss zu Beginn des Bruchversuchs wächst dieser Riss weiter in die auf der linken Seite benachbarte Ti-Schicht. Dieses Risswachstum wird begleitet von einer erhöhten Versetzungsaktivität in der Nähe der Rissspitze. Jedoch werden zusätzlich Versetzungsaktivitäten im Radius von ca. $450 \mathrm{~nm}$ um die Rissspitze festgestellt.

Im vierten und fünften Schritt wird die Ti-Schicht, in der der Riss liegt, weiter verformt und letztendlich wächst der Riss, wie auch bei den anderen Lamellen dieser Ti-Schichtdicke ohne Gasatmosphäre, in der Ti-Schicht weiter. In Abbildung 4.65c ist eine Hellfeldaufnahme am Ende des fünften Verformungsschrittes gezeigt. Es ist zu erkennen, wie sich der Riss von der Kerbe aus in die Ti-Schicht und dann weiter in dieser bewegt hat.

Im sechsten und siebten Schritt wächst der Riss kontinuierlich weiter in der bereits gerissenen Ti-Schicht, bis es zum katastrophalen Versagen der Lamelle kommt. Bevor der Riss unkontrolliert durch die gesamte Lamelle wächst, ist zu erkennen, dass sich die Ausbreitungsrichtung des Risses geringfügig in Richtung der auf der rechten Seite benachbarten $\mathrm{ZrO}_{2}$-Schicht orientiert. Die $\mathrm{ZrO}_{2}$-Schicht wird jedoch nicht erreicht und daher auch nicht verformt. In diesem Bereich können keine Veränderungen in der Tiund der $\mathrm{ZrO}_{2}$-Schicht beobachtet werden, die diese Umorientierung erklären könnten. In Abbildung 4.65d ist eine Dunkelfeldübersichtsaufnahme nach dem unkontrollierten Risswachstum gezeigt. In der Dunkelfeldaufnahme, aber auch in den beiden Hellfeldaufnahmen in der Nähe der Kerbe in höherer Vergrößerung (Abbildungen 4.65e und 4.65f), ist eindeutig zu erkennen, dass sich der Riss, der sich während des katastro- 


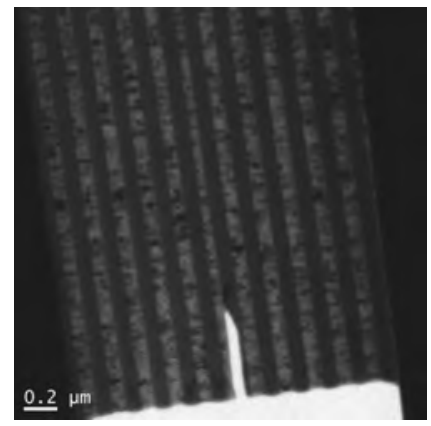

(a) BF Übersicht

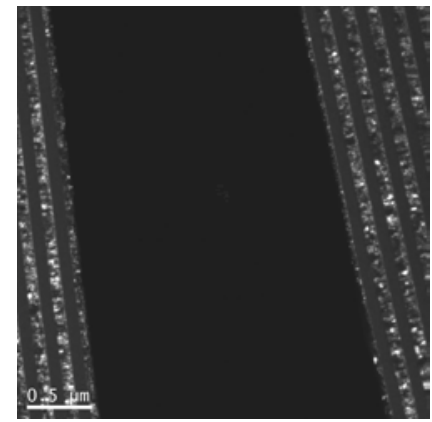

(d) DF Riss Ende

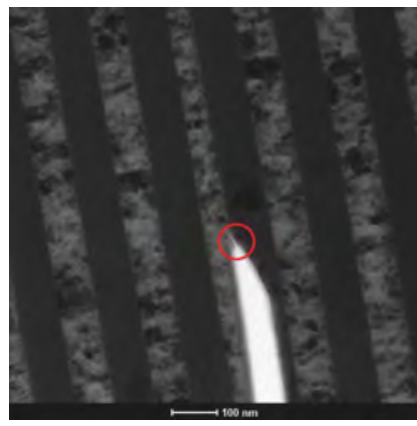

(b) BF Kerbe

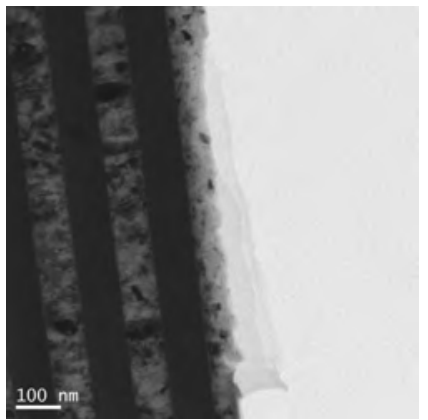

(e) BF links

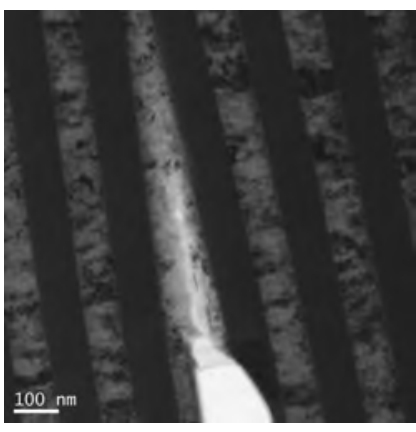

(c) BF Riss 5. Schritt

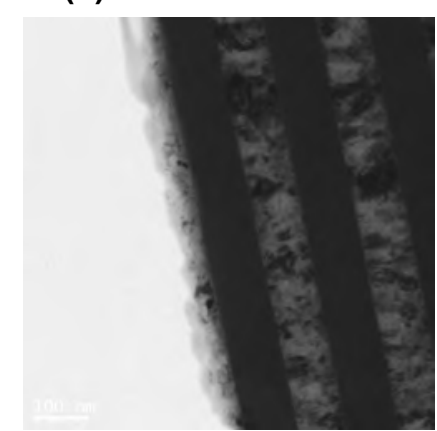

(f) BF rechts

Abbildung 4.65: Bruchversuch Ti100/ZrO 100 entlang der Grenzflächen Wasser-Atmosphäre

\begin{tabular}{ccc}
$r_{p}[\mathrm{~nm}]$ & $J_{I Q}\left[\mathrm{~J} \mathrm{~m}^{-2}\right]$ & $K_{I Q}\left[\mathrm{MPa} \mathrm{m}^{1 / 2}\right]$ \\
\hline \hline $524(44)$ & $11,087(427)$ & $1,323(128)$
\end{tabular}

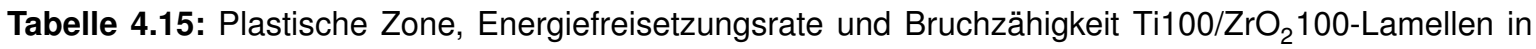
$\mathrm{H}_{2} \mathrm{O}$

phalen Versagens der Lamelle gebildet hat, in einer Ti-Schicht ausgebreitet hat. Ein Scherband kann nicht beobachtet werden.

Eine zweite Lamelle, die in Wasser-Atmosphäre getestet wird, zeigt ein vergleichbares Bruchverhalten mit einem Risswachstum in der Mitte einer Ti-Schicht ausgehend von der in einer Ti-Schicht liegenden Spitze der Kerbe. Diese Lamelle unterstützt damit das bereits beschriebene Bruchverhalten. Sie versagt während des Bruchversuchs an der Kontaktfläche zu einer der Si-Säulen.

In Tabelle 4.15 sind die Größe der plastischen Zone und die kritischen Werte der Energiefreisetzungsrate und der Bruchzähigkeit dargestellt. Die Bruchzähigkeit wird mit dem Elastizitätsmodul der $100 \mathrm{~nm}$ dicken Ti-Schichten berechnet.

\subsubsection{EELS Analyse in der Gasatmosphäre}

Anhand der EELS-Analysen in der jeweiligen Gas-Atmosphäre soll herausgefunden werden, ob das geänderte Bruchverhalten in Verbindung mit einer Veränderung des 


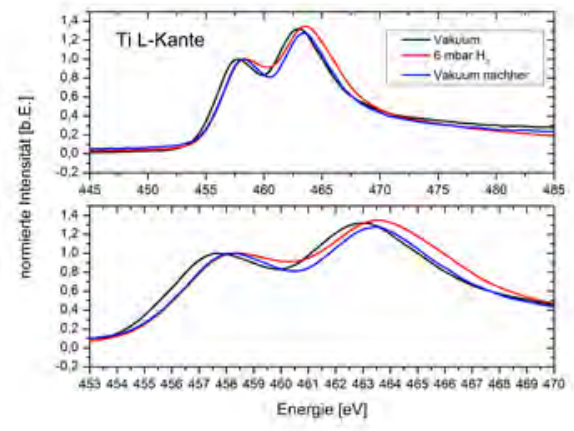

(a) Ti L-Kante

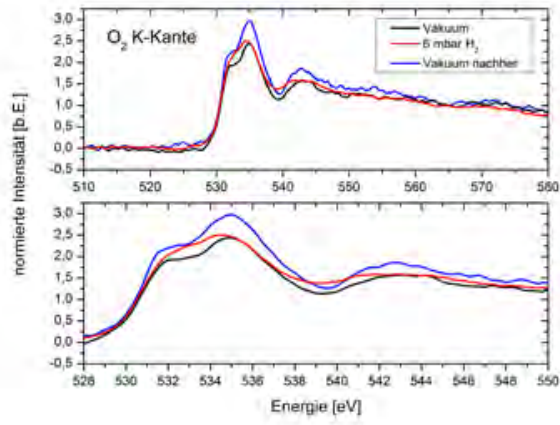

(b) O K-Kante

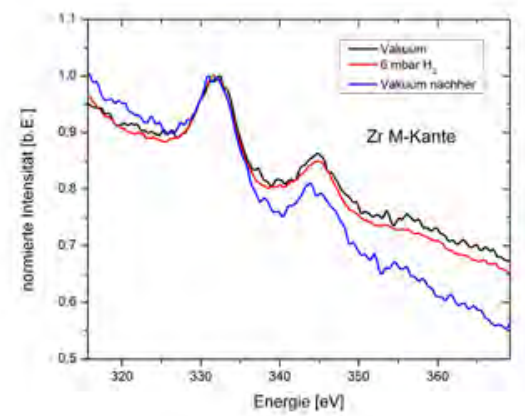

(c) Zr M-Kante

Abbildung 4.66: EELS-Analyse in Wasserstoff-Atmosphäre

Materials steht. Die EELS-Spektren werden sowohl im Vakuum als auch in der GasAtmosphäre aufgenommen und miteinander verglichen. Es wird für Zirkon die MKante, für Titan die L-Kante und für Sauerstoff die K-Kante analysiert. Alle abgebildeten Spektren wurden im Dual-EELS-Modus aufgenommen und damit die Energieskala anhand des Nullverlustmaximums geeicht. Zum besseren Vergleich sind die Intensitäten normiert. Für Titan erfolgt die Normierung anhand der $L_{3}$-Kante, für Zirkon anhand der $M_{3}$-Kante und für Sauerstoff bei einer Energie von $560 \mathrm{eV}$.

\section{Wasserstoff-Atmosphäre}

In Abbildung 4.66 sind exemplarisch die EELS-Spektren in einer WasserstoffAtmosphäre gezeigt. Die Ti L-Kante in Abbildung 4.66a zeigt eine Verschiebung zu höheren Energieverlusten, die auch nach dem Entfernen des Wasserstoffs erhalten bleibt. Die Verschiebung beträgt etwa 0,6 eV. Dem hingegen können in den O- und den $\mathrm{Zr}$ Spektren keine signifikanten Veränderungen in den Energien der Peakpositionen oder der Form der Spektren beobachtet werden (vgl. Abbildungen 4.66b und 4.66c). Diese EELS-Spektren sind an einer FIB-Lamelle, die speziell für diese Untersuchung hergestellt wird, aufgenommen. An den Lamellen der Bruchversuche in der Wasserstoff- 


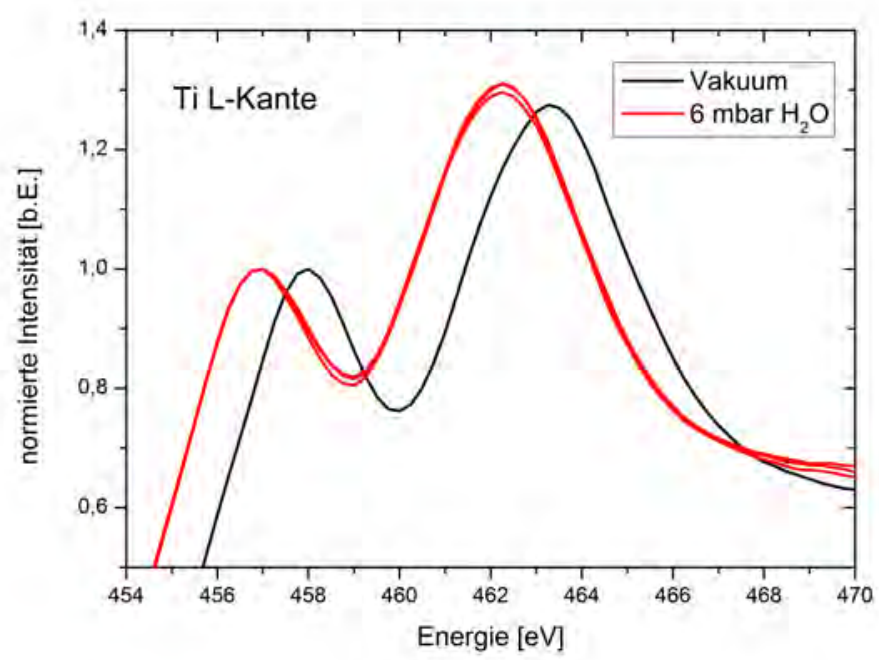

Abbildung 4.67: EELS-Analyse in Wasser-Atmosphäre

Atmosphäre können auch EELS-Spektren aufgenommen werden, deren Qualität nicht ausreicht, um eine klare Aussage zu erhalten. Die EELS-Spektren der Bruchversuchslamellen zeigen in einem Fall einen vergleichbaren Trend der Energieverschiebung der Ti L-Kante. Die zweite Bruchversuchslamelle hingegen zeigt keine Veränderung in der Ti L-Kante unter Wasserstoff-Einfluss.

\section{Wasser-Atmosphäre}

Die EELS-Analyse in Wasser-Atmosphäre zeigt wie schon im Fall des Wasserstoffs keine Veränderungen in den Zirkon- und Sauerstoff-Spektren (hier nicht gezeigt). Die Ti L-Kante hingegen zeigt eine Verschiebung zu geringeren Energien (vgl. Abbildung 4.67). Die Verschiebung beträgt hier konsistent etwa $1 \mathrm{eV}$. 


\section{Diskussion}

Im nachfolgenden Kapitel werden die gewonnenen Ergebnisse bezüglich der Rissbildung und Rissbewegung in den Lamellen diskutiert. Der Effekt der Längenskala der Ti-Schichtdicken auf das Bruchverhalten steht hier im Vordergrund, mögliche Erklärungsansätze werden ausführlich beschrieben. Abschließend werden zusätzlich die Ergebnisse der Bruchversuche in einer Gasatmosphäre im Hinblick auf das veränderte Bruchverhalten erläutert.

Für die in-situ Bruchversuche bleibt zusammenfassend festzuhalten, dass die hohen Festigkeiten der Multilagen gerade bei dünnen Ti-Schichtdicken und die besonderen Eigenschaften der kristallin/amorphen Grenzflächen einen entscheidenden Einfluss auf das Bruchverhalten haben. Die starken Grenzflächen mit Festigkeiten im Bereich von etwa 3 GPa stellen keine Schwachstelle für den Bruch dar und es ist zu erwarten, dass andere Schwachstellen zuerst versagen werden. Erst wenn die Festigkeit der Ti-Schicht die Grenzflächenfestigkeit überschreitet, versagen die Grenzflächen (vgl. Abschnitt 4.4.3). Neben der Grenzflächenfestigkeit kann die Rauigkeit der Grenzflächen ebenfalls von Bedeutung sein. In der Literatur wird berichtet, dass eine rauere Grenzfläche einen höheren Widerstand ( $\approx 50 \%$ größere Bruchenergie der Grenzfläche für $\mathrm{SiO}_{2} / \mathrm{TiN}$ ) gegenüber einer Delamination oder Rissbildung hat Dau98. Die HRTEM-Analysen sowie vorangegangene TEM- und XRR-Untersuchungen Lie07, Kno12, Lie12, haben eine Rauigkeit von etwa einem Nanometer bestätigt. Da die Rauigkeit der Grenzflächen in den $\mathrm{Ti} / \mathrm{ZrO}_{2}$-Multilagen für alle Schichtdickenkombinationen etwa gleich groß ist, kann angenommen werden, dass die Rauigkeit das längenskalenabhängige Bruchverhalten der Multilagen nicht maßgeblich beeinflusst. Inwiefern die Rauigkeit sich auf die kritische Bruchzähigkeit oder die Größe der plastischen Zone auswirkt, kann aufgrund fehlender Vergleichsdaten mit variierender Rauigkeit nicht bestimmt werden.

Der Vergleich zweier Multilagensysteme identischer Materialien aber unterschiedlicher Grenzflächengeometrie zeigt, dass neben den mechanischen und den chemischen Eigenschaften der Grenzflächen oder der jeweiligen Einzelschichten auch die Geometrie der 
gesamten Multilage von Interesse für das Bruchverhalten ist [Liu96]. Die in dieser Veröffentlichung möglichst perfekt präparierte Multilage mit parallelen Grenzflächen versagt in Biegeversuchen vergleichbar mit den in dieser Dissertation untersuchten Multilagen. In einer Multilagenstruktur mit nicht parallelen Grenzflächen breitet sich der Riss auch in der Grenzfläche aus. Dieses veränderte Verhalten ergibt sich durch eine hinreichend hohe Scherkomponente der Spannungen in den Multilagen mit nicht parallelen Grenzflächen, die die kritische Spannung zur Delamination der Grenzfläche überschreiten kann. Für den in dieser Dissertation vorliegenden Fall bleibt anhand der Erkenntnisse aus [Liu96] festzuhalten, dass aufgrund der sehr guten Parallelität der Einzelschichten und Grenzflächen der $\mathrm{Ti} / \mathrm{ZrO}_{2}$-Multilagen nicht zu erwarten ist, dass der Riss allein durch die Geometrie der Grenzflächen auf die Grenzflächenebene abgelenkt wird.

Der Einfluss der intrinsischen Spannungen durch die Schichtherstellung auf die Wahl des Risspfades wird für den in dieser Arbeit vorliegenden Fall der Ti/ $\mathrm{ZrO}_{2}-$ Multilagen als weniger wichtig eingestuft. Da alle Proben ohne eine zusätzliche Erwärmung des Substrates hergestellt werden, um die amorphe Struktur des $\mathrm{ZrO}_{2}$ zu ermöglichen, ist nicht zu erwarten, dass hohe intrinsische Spannungen in den Schichten vorliegen.

Im Folgenden wird besonders auf die Randbedingungen, die durch die Multilagenstruktur erzeugt werden, und deren Einfluss auf das Bruchverhalten bzw. die plastische Verformung der einzelnen Schichten eingegangen. Die Ergebnisse werden auf der Basis der in dem Abschnitt 2.1.1 beschriebenen möglichen Längenskaleneffekten (Griffith's Kriterium, Unempfindlichkeit gegenüber Schwachstellen, Rissspitzenabschirmung, längenskalenabhängige Festigkeiten, Abrundung der Rissspitze und Einschränkung der plastischen Zone) diskutiert. Abschließend werden die Abhängigkeit der Größe der plastischen Zone sowie die der kritischen Bruchzähigkeit von der Ti-Schichtdicke zur Diskussion gestellt.

\subsection{Einzelschichten}

\subsubsection{Titan-Einzelschichten}

Die verschiedenen TEM-Aufnahmen sowie das Bruchverhalten bzw. die mikrostrukturellen Prozesse während des Risswachstums zeigen die typischen Merkmale eines duktilen Versagens, wie zum Beispiel die Einschnürung des Materials direkt in der Nähe der Rissspitze als auch die Beobachtung der Rissbrückenbildung. Sowohl für die Einschnürungen als auch für die Rissbrücken wird das Material stark gedehnt, ohne dass es reißt. Weiterhin kann während des Bruchversuchs anhand der Kontrastwechsel eine intensive Aktivität von Versetzungen in ausgewählten Körnern beobachtet werden. Ob dies die Nukleation von neuen Versetzungen oder eine Bewegung der bereits im Material vorhandenen Versetzungen ist, kann nicht mit Sicherheit unterschieden 
werden.

Die Beobachtung, dass nur ausgewählte Körner Versetzungsaktivität zeigen, ist durch die Variation in der Korngröße zu begründen. Aufgrund der längenskalenabhängigen Fließspannung des Titans Kno12 wird in größeren Körnern die notwendige Spannung zur Bewegung und/oder Nukleation von Versetzungen früher erreicht. Da die konkrete Orientierung der Körner nicht bekannt ist, kann zusätzlich nicht ausgeschlossen werden, dass aufgrund der polykristallinen Mikrostruktur der Ti-Einzelschichten, die Körner mit stärkerer Versetzungsaktivität günstiger zu der Belastungsrichtung ausgerichtet sind.

Auch eine Rotation der Körner unter dem Einfluss der äußeren Spannung ist als Verformungsmechanismus in nanokristallinen Materialien bekannt, wie sie beispielsweise in freistehenden Gold-Schichten beobachtet werden kann [Mer14]. Da die notwendige Formveränderung der Körner bei einer Kornrotation typischerweise ein Diffusionsprozess ist [Sha04, ist zu erwarten, dass aufgrund der deutlich höheren Schmelztemperatur des Titans $(\approx 1900 \mathrm{~K}$ Cah83 $)$ im Vergleich zu Gold $(\approx 1300 \mathrm{~K}$ Cah83) die Kornrotation in den Ti-Schichten nicht der bestimmende Verformungsmechanismus ist Wei05. Weiterhin liefert die Beobachtung linienhafter Kontrastwechsel in den Ti-Körnern einen deutlichen Hinweis darauf, dass die Versetzungsbewegung bzw. -nukleation als eher wahrscheinlich anzusehen ist.

Zweifelsfrei festzuhalten ist, dass es eine stark ausgeprägte Plastizität im Bereich der Kontrastwechsel in der Nähe der Rissspitze gibt, die einen Einfluss auf den ablaufenden Rissmechanismus hat (vgl. Abschnitt 2.1.1).

In der Literatur wird berichtet, dass ein Wechsel des Rissmechanismus hin zu sprödem Versagen in kleineren Körnern und der damit verknüpften Möglichkeit, Versetzungen zu nukleieren oder bewegen zu können, auftreten kann Ovi07, (experimentelle Beobachtungen in Ni bzw. Ni-Legierung [Li04, Li05], Cu oder AlLegierung [You04, You05, Che05a, You06] und Al- oder Au-Filme [Haq04]).

Die Korngröße der Ti-Einzelschichten liegt im Bereich von etwa $35 \mathrm{~nm}$ bis $100 \mathrm{~nm}$, die größer als die kritische Korngröße $(10 \mathrm{~nm}$ bis $30 \mathrm{~nm})$ für einen Übergang zu sprödem Versagen ist, sodass die Beobachtung des duktilen Versagens in den Bruchversuchen für die Orientierung senkrecht zur Wachstumsrichtung anhand der Literatur bestätigt werden kann Kum03, Sin11.

Durch eine geringe Korngröße wird zum einen die Fließspannung erhöht und zum anderen die plastische Zone eingeschränkt (vgl. Abschnitt 2.1.1). Diese beiden Mechanismen erhöhen im Allgemeinen die Wahrscheinlichkeit, dass das verformte Material eher spröde versagt. Da präparationsbedingt davon auszugehen ist, dass die Ti-Körner bereits Versetzungen enthalten, müssen diese während der Verformungen nicht neu nukleiert werden. Durch die Bewegung dieser Versetzungen kann die Spannungskonzentration in der Nähe der Rissspitze oder der Korngrenzen ( Ovi07], 


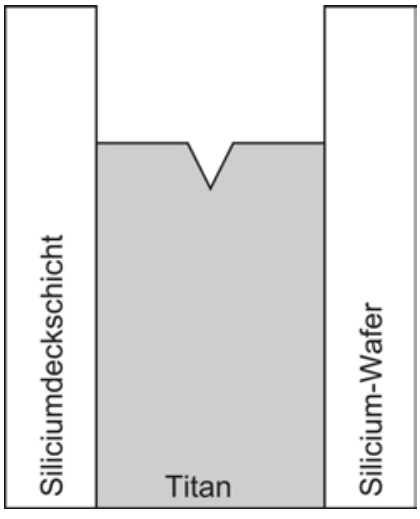

(a) senkrecht

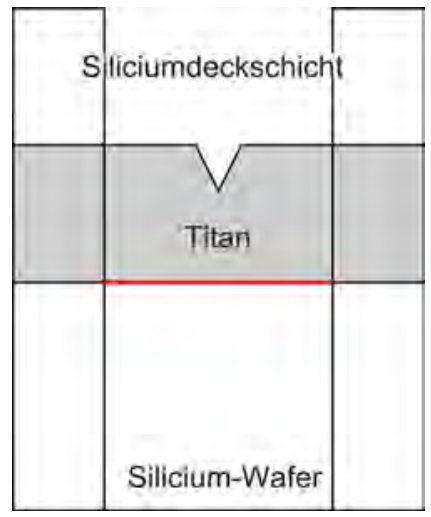

(b) parallel

Abbildung 5.1: Skizze zur Bruchgeometrie der Ti-Einzelschichten

vgl. Abbildung 2.3a abgebaut werden. Somit kann Verformungsenergie dissipiert werden und das Bruchverhalten ist eher duktil.

Dem hingegen versagt die Titan-Einzelschicht, die parallel zu ihrer ursprünglichen Wachstumsrichtung getestet wird, spröde. Wie bereits für die Ti-Einzelschicht in der Orientierung senkrecht zur Wachstumsrichtung diskutiert, ist zu erwarten, dass sich aufgrund der vergleichbaren Mikrostruktur ein ähnliches Bruchverhalten zeigen sollte. Es ist daher wahrscheinlich, dass das veränderte Bruchverhalten der gedrehten Schicht nicht durch mikrostrukturelle oder materialspezifische Veränderungen in der Ti-Schicht zu erklären ist. Vielmehr wird die geänderte Geometrie des gesamten Bruchversuchs als Grund für das schnelle, eher spröde Versagen der Lamelle angesehen.

Anhand der Skizzen in Abbildung 5.1 ist eindeutig der Unterschied in der Geometrie der Lamellen zu erkennen. Für den Fall der Ausrichtung senkrecht zur Wachstumsrichtung (5.1a) ist die Ti-Schicht zwischen den beiden Si-Säulen eingeschlossen und der Riss könnte sich „unendlich“ weit ausbreiten. Die gedrehte Geometrie für die Bruchversuche parallel zur Wachstumsrichtung unterscheidet sich hier grundlegend. Aufgrund der Geometrie kann der Riss während seines Wachstums auf die Grenzfläche zwischen der Ti-Schicht und dem Si-Substrat treffen (rote Linie in 5.1b) und müsste dort in das spröde Silicium wachsen. Während des Bruchversuchs wird nicht nur die Ti-Schicht unter dem Einfluss der extern aufgebrachten Spannung verformt, sondern auch der Si-Wafer unterhalb der Ti-Schicht. Es ist daher anzunehmen, dass auch in dem Si-Wafer an beispielsweise Fremdatomen, Leerstellen oder Störungen eine Rissbildung stattfinden könnte. Da Silicium im Allgemeinen spröde versagt und eine geringere Bruchzähigkeit aufweist als zum Beispiel Metalle, kann es in dem Si-Wafer zu einem katastrophalen Versagen kommen, wenn ein Riss gebildet wird. Durch einen solchen Riss im Silicium entsteht eine Instabilität, die durch die dünne Ti-Schicht nicht kompensiert werden kann und diese daher auch katastrophal versagt.

Da die Elastizitätsmoduln von Titan $(\approx 150 \mathrm{GPa}$ Kno12] $)$ und Silicium 


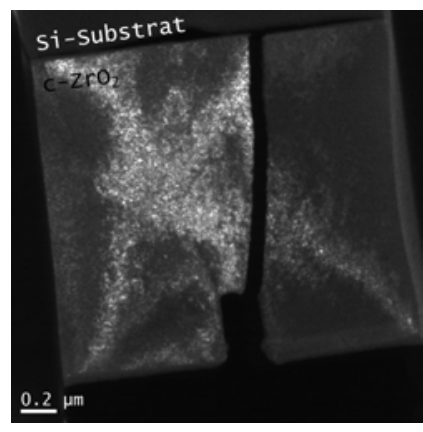

(a) kristallin

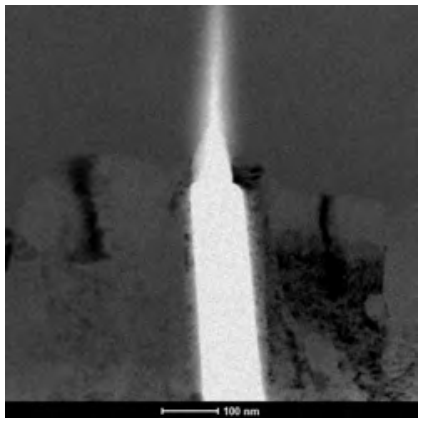

(b) amorph

Abbildung 5.2: Gegenüberstellung des Bruchverhaltens der kristallinen und amorphen $\mathrm{ZrO}_{2}$-Schichten

(130 GPa bis $189 \mathrm{GPa}$ Wor65 je nach Kristallorientierung) relativ ähnlich sind, ist nicht zu erwarten, dass allein eine Asymmetrie in den mechanischen Eigenschaften das katastrophale Versagen bewirkt. Vielmehr wurde während der Probenpräparation mehrfach beobachtet, dass die Grenzfläche zwischen der Ti-Schicht und dem Si-Wafer nicht besonders stark ist. Speziell das mechanische Polieren der Proben hat oft dazu geführt, dass die Ti-Schicht von dem Si-Wafer abgelöst wurde, sodass an der Oberfläche des Si-Wafers an atomaren Stufen eine Rissbildung möglich ist, die das katastrophale Versagen der Lamellen verursacht. Für die Multilagen-Proben, bei denen das amorphe $\mathrm{ZrO}_{2}$ zuerst auf den Si-Wafer aufgebracht wurde, konnte eine Ablösung der Schichten nicht beobachtet werden.

\subsubsection{Zirkonoxid-Einzelschichten}

Das beschriebene Bruchverhalten der ausgelagerten und somit kristallinen $\mathrm{ZrO}_{2^{-}}$ Schicht (vgl. Abschnitt 4.3.2) spiegelt die Erwartungen eines Bruchverhaltens einer spröden Probe wie zum Beispiel von Keramiken oder von oxidischen Gläsern wider Ovi11, Ovi15. So zeigt Silicium sowohl in den hier realisierten in-situ TEM-Bruchversuchen als auch in anderen Geometrien (Dimensionen auf der Mikrometerskala) ein vergleichbares, unkontrolliertes sprödes Verhalten [Jay15].

Die amorphe $\mathrm{ZrO}_{2}$-Einzelschicht hingegen zeigt $\mathrm{zu}$ Beginn des Risswachstums ein leicht verändertes Bruchverhalten. Es kann eine lokale Einschnürung der Schicht vor der Spitze der Kerbe bzw. des Risses beobachtet werden (hellerer Kontrast in TEM-Hellfeldabbildungen, vgl. Abbildung 5.2b), die auf eine lokal duktile Verformung der amorphen $\mathrm{ZrO}_{2}$-Schicht schließen lässt, bevor sie letztlich spröde versagt.

In Mikrodruckversuchen Kno12 und Indentationsexperimenten Hei11b, Kno12 amorpher $\mathrm{ZrO}_{2}$-Schichten konnte eindeutig eine lokale Bildung von Scherbändern nachgewiesen werden, die als typischer Verformungsmechanismus in amorphen Mate- 


\begin{tabular}{ccc|cc|} 
& \multicolumn{2}{c|}{ Hei11b } & \multicolumn{2}{c|}{ Kno12 } \\
& $H[\mathrm{MPa}]$ & $E_{r}[\mathrm{GPa}]$ & $H[\mathrm{MPa}]$ & $E_{r}[\mathrm{GPa}]$ \\
\hline${\mathrm{c}-\mathrm{ZrO}_{2}}$ & 16,0 & 260,9 & - & - \\
${\mathrm{a}-\mathrm{ZrO}_{2}}$ & 11,5 & 229,4 & 13,2 & 190
\end{tabular}

Tabelle 5.1: Mechanische Eigenschaften der $\mathrm{ZrO}_{2}$-Schichten aus der Literatur Hei11b Kno12,

rialien angesehen wird (z.B. Mos06b, Vol08 für amorphe Metalle) und ebenfalls einen Hinweis auf eine duktile Verformung liefert.

Die Bruchversuche der Multilagenproben zeigen Scherbänder in den $\mathrm{ZrO}_{2}$-Schichten (vgl. Abbildung 4.22). In den Bruchversuchen der amorphen $\mathrm{ZrO}_{2}$-Einzelschichten können keine Hinweise auf Scherbänder gefunden werden. Dieser Unterschied im Bruchverhalten erklärt sich anhand der fehlenden Randbedingungen in den Einzelschichten. Die Grenzflächen zu den Ti-Schichten in den Multilagen können durch die aufgebrachte Spannung an den Grenzflächen Scherkomponenten erzeugen, die eine Scherbandbildung in den $\mathrm{ZrO}_{2}$-Schichten ermöglichen. Dem hingegen entsprechen die Bruchversuche in der Einzelschichtgeometrie einem Mode I-Bruchversuch, der keine oder nur geringe Scherkomponenten aufweist, die eine Scherbandbildung begünstigen würden.

Im Vergleich der in der Literatur berichteten mechanischen Eigenschaften zwischen amorphem und kristallinem Zirkonoxid weist die amorphe Schicht sowohl eine geringere Härte als auch einen geringeren Elastizitätsmodul auf [Hei11b] (vgl. Tabelle 5.1). Im amorphen Zustand zeigt das $\mathrm{ZrO}_{2}$ in Indentationsexperimenten einen duktilen Verformungsmechanismus, wobei in der gewählten Bruchgeometrie nach der anfänglichen Einschnürung ein sprödes Risswachstum beobachtet wird. Durch die amorphe Struktur wird eine lokale, plastische Verformung ermöglicht, sodass das Bruchverhalten zu Beginn einen duktilen Charakter aufweist.

\subsection{Bruchversuche der Multilagen}

\subsubsection{Entlang der Grenzflächen}

Die Bruchversuche in der Geometrie, die die Rissausbreitung parallel zu den Grenzflächen ermöglicht, zeigen eindeutig ein variiertes Bruchverhalten mit Abhängigkeit von der Ti-Schichtdicke.

Für die 100 nm-dicken Ti-Schichten wird für alle durchgeführten Bruchversuche ein stabiles Risswachstum in den Ti-Schichten beobachtet. Auch in uniaxialen Zugversuchen zeigen die Ti100/ZrO $100-P r o b e n$ ein Versagen in der Ti-Schicht (vgl. Anhang 
B). Die mittlere untersuchte Ti-Schichtdicke von $50 \mathrm{~nm}$ zeigt auch, wie die dickeren TiSchichten, Risswachstum in den Ti-Schichten. Erst bei der dünnsten Ti-Schichtdicke von $10 \mathrm{~nm}$ zeigt sich ein signifikanter Unterschied. Es kann zur Rissausbreitung an der kristallin/amorphen Grenzfläche kommen.

Im Vergleich mit den Mikrokompressionsversuchen an 45-verkippten Grenzflächen relativ zur Belastungsrichtung $[$ Kno12 zeigt sich ein konsistentes Bild.

Durch die Unterschiede in den elastischen Konstanten zwischen zwei isotropen, linear elastischen Materialien (Materialien 1 und 2 oder einer Dünnschicht 2 auf einem Substrat 1) kann vorhergesagt werden, in welchem der beiden Materialien der Riss entsteht oder ob er entlang der Grenzfläche läuft Dun69]. Der Einfluss des elastischen Unterschiedes auf das Spannungsfeld in der Nähe der Rissspitze wird durch die zwei Dundurs Parameter $\alpha$ und $\beta$ ausgedrückt. Es kann zu einer Rotation/Oszillation der Spannungsverteilung (beschrieben über $\beta$ ) an der Rissspitze kommen, sodass eine Scherspannung (Mode II, Spannungskomponente senkrecht zu den Grenzflächen) entsteht, die einen Riss ablenken kann Dun69, Rit93, McN96, Lan03. Die Parameter berechnen sich über die folgenden Gleichungen und betragen für das Ti/ZrO${ }_{2}$-System (Material $1=\mathrm{ZrO}_{2}$, Material $2=\mathrm{Ti}$ )

$$
\begin{aligned}
\alpha & =\frac{E_{1}-E_{2}}{E_{1}+E_{2}} \approx 0,21 \\
\beta & =\frac{1}{2} \frac{\mu_{1}\left(1-2 \nu_{2}\right)-\mu_{2}\left(1-2 \nu_{1}\right)}{\mu_{1}\left(1-\nu_{2}\right)+\mu_{2}\left(1-\nu_{1}\right)} \approx 0,02
\end{aligned}
$$

wobei $E$ der Elastizitätsmodul (aus Tabelle 3.2), $\mu$ der Schermodul und $\nu$ die Poissonzahl der einzelnen Materialien sind 1

Es gilt im Allgemeinen, dass Risse in der steiferen Schicht die Tendenz haben hin zur Grenzfläche bzw. in die nachgiebigere Schicht abgelenkt zu werden, wenn $\beta>0$ (vgl. Abbildung 5.3a). Für $\beta<0$ tendieren sie in die steifere Schicht hinein (siehe Abbildung $5.3 \mathrm{~b}$. Die berechneten Dundurs Parameter für das $\mathrm{Ti} / \mathrm{ZrO}_{2}-$ System zeigen typische Größen im Vergleich mit berichteten Parametern für Metall/Keramik-Grenzflächen in der Literatur $(\alpha<0,6$ und $\beta<0.1)$ [Sch92], sodass die berechneten Parameter für das $\mathrm{Ti} / \mathrm{ZrO}_{2}$-System als belastbar angesehen werden.

Im Vergleich mit den Bruchversuchen der Ti100/ $\mathrm{ZrO}_{2}$ 100-Proben zeigt sich eine klare Übereinstimmung mit den Vorhersagen der Dundurs Parameter. Da beide Dundurs Parameter für die $\mathrm{Ti} / \mathrm{ZrO}_{2}$-Proben einen positiven Wert liefern, ist zu erwarten, dass es entsprechend der Vorhersagen zu einem stabilen Risswachstum in der Ti-Schicht kommt. Ein ursprünglich in der steiferen $\mathrm{ZrO}_{2}$-Schicht gebildeter Riss wird hin zur TiSchicht abgelenkt und breitet sich in deren Mitte aus (vgl. Abschnitt 4.4.1). Auch für

\footnotetext{
${ }^{1}$ Schermodul und Poissonzahl entnommen aus http://www.azom.com/article.aspx?ArticleID= 9118 für Ti und aus http://www.azom.com/article.aspx?ArticleID=133 für $\mathrm{ZrO}_{2}$
} 


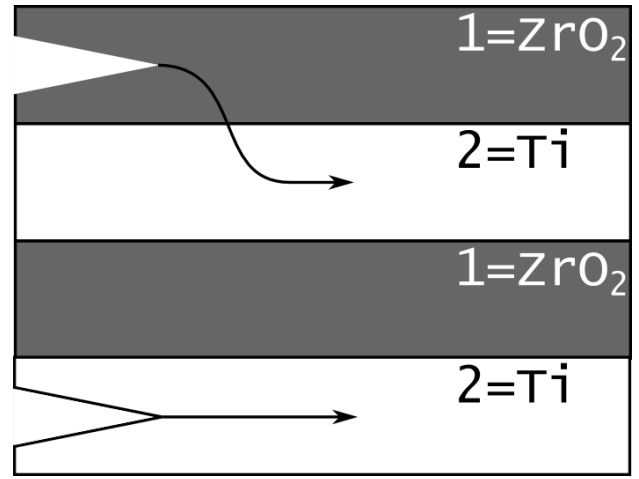

(a) $\beta>0$

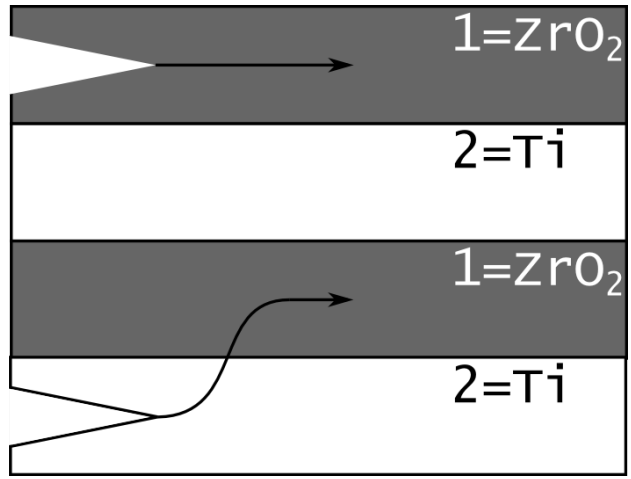

(b) $\beta<0$

Abbildung 5.3: Schematische Darstellung der Auswirkungen der Dundurs Paramter für das $\mathrm{Ti}^{-} \mathrm{ZrO}_{2}-$ System

die $\mathrm{Ti50} / \mathrm{ZrO}_{2}$ 100-Proben stimmt das experimentell beobachtete Verhalten mit den theoretischen Erwartungen überein. Erst bei der kleinsten Ti-Schicht zeigt sich eine Abweichung von den Vorhersagen, die später genauer diskutiert wird.

Die Wahl des Risspfades stimmt nicht immer mit den Vorhersagen aus den elastischen Unterschieden überein Mat12]. Beispielsweise ist zu erwarten, dass eine Grenzfläche, die eine geringere Bruchzähigkeit bzw. Festigkeit hat als die beteiligten Materialien, immer zuerst versagt unabhängig von den elastischen Unterschieden der Materialien Fle91, Hut92]. Ein weiterer Effekt, der sich nicht allein durch den Unterschied der elastischen Konstanten erklären lässt, tritt dann auf, wenn die plastische Zone größer als die Metallschichtdicke in einer Multilagenstruktur ist [Kru04]. Die Randbedingungen, die durch die Grenzflächen zu den benachbarten Schichten erzeugt werden, limitieren die Plastizität in dünneren Metallschichten stärker im Vergleich zu Metallschichten, die größer als die eigentliche plastische Zone sind. Diese limitierte Plastizität bedingt ein weniger starkes Abrunden der Rissspitze, wodurch höhere lokale Spannungen in der Nähe der Rissspitze erreicht werden (vgl. Abschnitt 2.1.1). Mit höheren Spannungen steigt die Wahrscheinlichkeit einer Rissbildung in der benachbarten Schicht, sodass entgegen der Dundurs Parameter der Riss unter Umständen in die steifere Schicht oder zur Grenzfläche abgelenkt werden kann.

In allen Fällen der $\mathrm{Ti} / \mathrm{ZrO}_{2}$-Multilagen überschreiten die Größen der plastischen Zone die Schichtdicken der Metallschichten deutlich. Jedoch wird für die Ti50/ZrO 100 und Ti100/ZrO 2 100-Proben keine Abweichung des Risspfades beobachtet, sodass die Plastizität in den dickeren Ti-Schichten noch ausreichend ist, um eine Abrundung der Rissspitze zu ermöglichen. Für die Ti10/ZrO 100 -Multilagen wird Risswachstum an der Grenzfläche beobachtet. Aufgrund der längenskalenabhängigen Fließspannung und der geringeren Duktilität (vgl. Abschnitt 2.1.1) ist die Plastizität stärker eingeschränkt, sodass höhere Spannungen in der Nähe der Rissspitze einen Beitrag dazu leisten, dass der Riss hin zur Grenzfläche abgelenkt wird. 
Für die Wahl des Risspfades gilt weiterhin, dass er entlang der Richtung, in der die höchste Triebkraft (Energiefreisetzungsrate) zur Rissbildung herrscht (in Richtungen in denen $K_{I I}=0$ ), oder entlang der mikrostrukturell schwächsten Richtung (zum Beispiel Grenzflächen oder Korngrenzen) verläuft. Wenn es eine schwache Grenzfläche gibt, versagt die Probe üblicherweise dort, unabhängig davon ob $\beta$ positiv oder negativ ist, also unabhängig von den elastischen Unterschieden der beiden Materialien.

Metall/Keramik Grenzflächen sind im Allgemeinen stark und widerstandsfähig, wenn sie „perfekt“ sind, also möglichst frei von Fehlstellen oder Fremdatomen Bag94,Bag96, Eva99. Wenn Fremdatome die Grenzfläche verunreinigen, wird die Arbeit zur Separation der Materialien im Wesentlichen durch die veränderten chemischen Eigenschaften der Grenzfläche und die atomaren Bindungsstärken bestimmt. Als Beispiel soll hier die Analyse von $\mathrm{Al}_{2} \mathrm{O}_{3} / \mathrm{Au}$-Grenzflächen genannt werden. Die stetige Zugabe von Kohlenstoff resultiert in einer dramatischen Reduktion der Grenzflächenbruchzähigkeit von $>250 \mathrm{~J} \mathrm{~m}^{-2}$ für die saubere Grenzfläche $\mathrm{zu} 2 \mathrm{~J} \mathrm{~m}^{-2}$ für die mit Kohlenstoff angereicherte Grenzfläche Lip98 (vgl. Abschnitt 2.3.1).

Die $\mathrm{Ti} / \mathrm{ZrO}_{2}$-Grenzflächen sind relativ glatt, jedoch weisen sie eine chemische Unschärfe auf, die abhängig von der Einzelschichtdicke der Multilagen ist (vgl. Tabelle 4.2). Mit geringerer Schichtdicke sinkt zwar die absolute chemische Unschärfe der Grenzflächen, relativ zu der Ti-Einzelschichtdicke gesehen ist bei kleineren Schichtdicken jedoch ein größerer Bereich der Schicht beeinflusst. Bei einer Unschärfe jeder Grenzfläche von etwa $1 \mathrm{~nm}$ entspricht das prozentual gesehen $20 \%$ der Ti-Einzelschichtdicke im Fall der Ti10/ZrO 2 100-Multilagen. Für die größte Ti-Einzelschichtdicke beträgt dieser Anteil nur etwa $6 \%$. Obwohl es im Bereich der Grenzflächen eine verunreinigte Zone gibt, zeigen die Grenzflächen eine hohe Festigkeit in den Mikrokompressionsversuchen und versagen in den Bruchversuchen erst bei den dünnsten getesteten Ti-Schichtdicken.

Eine Analyse der Bruchmechanismen für Metallschichten, die zwischen zwei elastische Materialien eingebettet sind, zeigt, dass durch die Randbedingungen höhere Spannungen in der Metallschicht erreicht werden können |Var91]. So steigt die Spannung vor einer Rissspitze in einer eingeschränkten Metallschicht kontinuierlich mit der aufgebrachten Spannung. Steigt in einem Metall/Keramikschichtsystem (vgl. Abbildung 5.4) die Steifigkeit der Keramik, wobei die Eigenschaften der Metallschicht unverändert bleiben, ist das Auftreten eines schnellen Risswachstums oder eines Ablösens der Schicht an der Grenzfläche wahrscheinlicher. Ein vergleichbarer Trend hin zu unkontrolliertem Risswachstum und Ablösen der Schicht wird beobachtet, wenn die Schichtdicke der Metallschicht geringer wird und die Eigenschaften der Keramik unbeeinflusst bleiben. Im Extremfall kann es aufgrund der hohen Spannungen in solchen beschränkten Systemen auch zur Spaltung der Keramik kommen |Var91.

Zusammenfassend bleibt festzuhalten, dass die experimentellen Beobachtungen des von der Ti-Schichtdicke abhängigen Bruchverhaltens im Vergleich mit den Erkenntnissen 


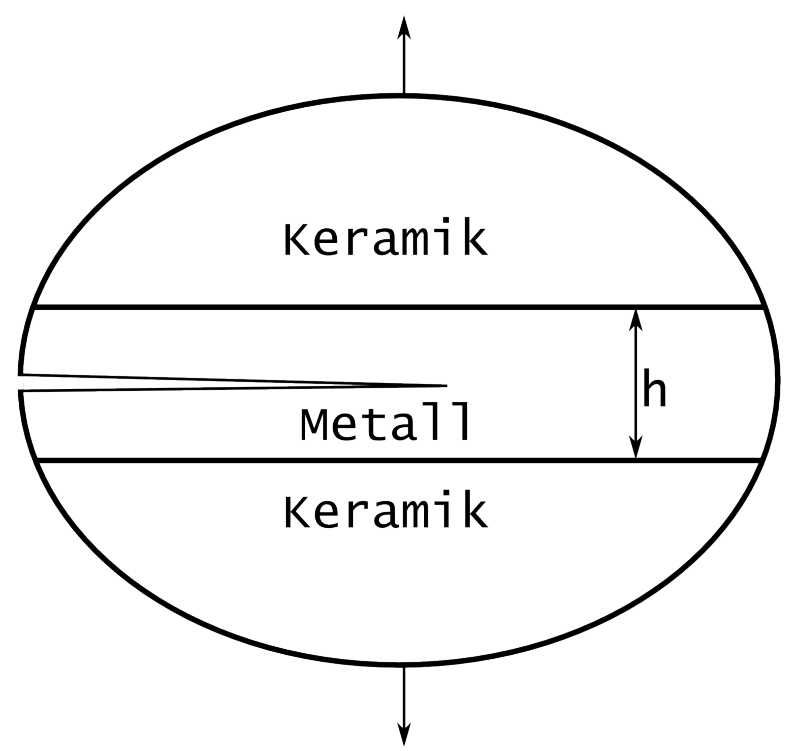

Abbildung 5.4: Metallschicht zwischen zwei Keramikschichten mit einem Riss in der Metallschicht nach [Var91]

aus der Literatur plausibel erscheinen. Anhand der theoretischen Voraussagen über das Risswachstum in Multilagen ist zu erwarten, dass sich für alle Ti-Schichtdicken der Riss, unabhängig davon wo er gebildet wird, hin zur Ti-Schicht und dort in der Mitte der Schicht ausbreitet. Für die beiden größeren untersuchten Ti-Schichtdicken ist dieses Verhalten so beobachtet worden. Die Abweichung von diesem theoretisch erwarteten Rissverlauf für die $10 \mathrm{~nm}$ dünnen Ti-Schichten erklärt sich durch die veränderten Verhältnisse der Festigkeiten der beiden Komponenten und der Grenzfläche.

Auch in einer komplexen Multilagenstruktur $\left(\mathrm{SiO} / \mathrm{TiN} / \mathrm{Ti} / \mathrm{TiN} / \mathrm{AlCu} / \mathrm{SiO}_{2}\right.$ ) wird eine Variation des Risspfades hin zu einer Ausbreitung an der Grenzfläche mit geringer werdender Schichtdicke der duktilen Komponenten beobachtet Dau98. Das veränderte Verhalten erklärt sich hier durch eine Variation des Bruchmodus aufgrund der elastischen Unterschiede der Materialien.

Die Beobachtung, dass der Riss nicht exklusiv entlang der Grenzfläche verläuft, lässt sich durch die polykristalline Mikrostruktur der Ti-Schichten nachvollziehen. Aufgrund der Anisotropie der mechanischen Eigenschaften der hexagonalen Kristallstruktur des Titans ist es möglich, dass lokal an einzelnen Körnern die Verhältnisse der lokalen Spannungen bzw. Festigkeiten (etwa 2,87 GPa) relativ zu der Grenzfläche (2,77 GPa) ein Risswachstum in der Ti-Schicht begünstigen. Die Anisotropie für das hexagonale Gitter des Titans kann einen Unterschied von maximal bis zu 30\% in den elastischen Konstanten bewirken. Es erscheint daher plausibel, dass es lokal durch die Anisotropie zu einem Wechsel der bevorzugten Rissausbreitung kommt, da sich die Festigkeiten der 10 nm-dünnen Ti-Schichten nur wenig von der gemessenen Grenzflächenfestigkeit unterscheidet. 


\subsubsection{Senkrecht zu den Grenzflächen}

Die experimentellen Beobachtungen der Bruchversuche in der Orientierung der Rissausbreitung senkrecht zu den Grenzflächen liefert für alle untersuchten Schichtdicken ein vergleichbares Verhalten. Die Rissausbreitung findet über alle Grenzflächen hinweg in Richtung des unterliegenden Si-Substrates statt. Die Proben mit den 100 nm-dicken Ti-Schichten versagen nach der Rissbildung in einer kontrollierten Weise über die Ausbildung von mehreren Rissbrücken in den Ti-Schichten. Für die beiden geringeren TiSchichtdicken werden weniger Rissbrücken in den Ti-Schichten gebildet.

Ausgehend von der linear-elastischen Bruchmechanik können laut Hua95] in Multilagen aus Metallen und Keramiken zwei miteinander in Konkurrenz stehende Mechanismen des Risswachstums unterschieden werden.

Ein Mechanismus beschreibt die wiederholte Renukleation eines Risses in der Keramikschicht, während die Metallschicht noch intakt bleibt. Die Metallschichten bilden dementsprechend Rissbrücken. An der Grenzfläche zur duktilen Metallschicht wird der Riss aufgehalten und rundet durch die plastische Verformung in der Metallschicht ab. Im Fortlauf des Risswachstums werden die einzelnen Risse in den Keramikschichten zu einem makroskopischen Riss zusammengeführt und die Probe versagt über die Rissausbreitung senkrecht zu den Grenzflächen (vgl. Abbildung 5.5 unten).

Der zweite mögliche Mechanismus beinhaltet die Nukleation von mehreren Rissen in der gleichen Keramikschicht in der Nähe der Rissspitze eines makroskopischen Risses (vgl. Abbildung 5.5 oben).

Der Wechsel im Bruchmechanismus wird durch ein kritisches Verhältnis der Schichtdicken der Metall- $\left(t_{m}\right)$ bzw. Keramikschicht $\left(t_{c}\right)$ bestimmt. Entscheidend für den Rissmechanimus ist das Verhältnis der Steifigkeiten der Metall- und der Keramikschicht.

$$
\left(\frac{t_{m}}{t_{c}}\right)_{k r i t .} \propto \frac{E_{c}}{E_{m}}
$$

Experimentelle Daten verschiedener Systeme $\left(\mathrm{Al} / \mathrm{Al}_{2} \mathrm{O}_{3}\right.$ und $\mathrm{Cu} / \mathrm{Al}_{2} \mathrm{O}_{3}$ mit verschiedenen Metall- und auch Keramikschichtdicken) zeigen, dass das kritische Schichtdickenverhältnis $\left(t_{m} / t_{c}\right)_{k r i t .}=2,5$ entspricht Hua95]. Das bedeutet, dass für Metallschichtdicken, die zweieinhalb mal dicker sind als die Keramikschicht, die Nukleation mehrerer Risse in der Keramikschicht auftreten sollte.

Anhand der gemessenen Elastizitätsmoduln der Komponenten des $\mathrm{Ti} / \mathrm{ZrO}_{2}$-Systems (vgl. Tabelle 3.2) ergibt sich ein Wert von etwa 1,25, der damit deutlich unterhalb des kritischen Wertes von 2,5 aus Hua95 liegt, was beutet, dass die Renukleation des Risses in der Keramikschicht der vorherrschende Mechanismus ist (vgl. Abbildung 5.5 unten). Diese Vorhersage kann durch die experimentellen Beobachtungen dieser Dissertation unterstützt werden (vgl. Abbildung 4.45d oder 4.49c). Sie gilt aber nicht universell, da der Einfluss der plastischen Verformung in der Metallschicht im Bereich 


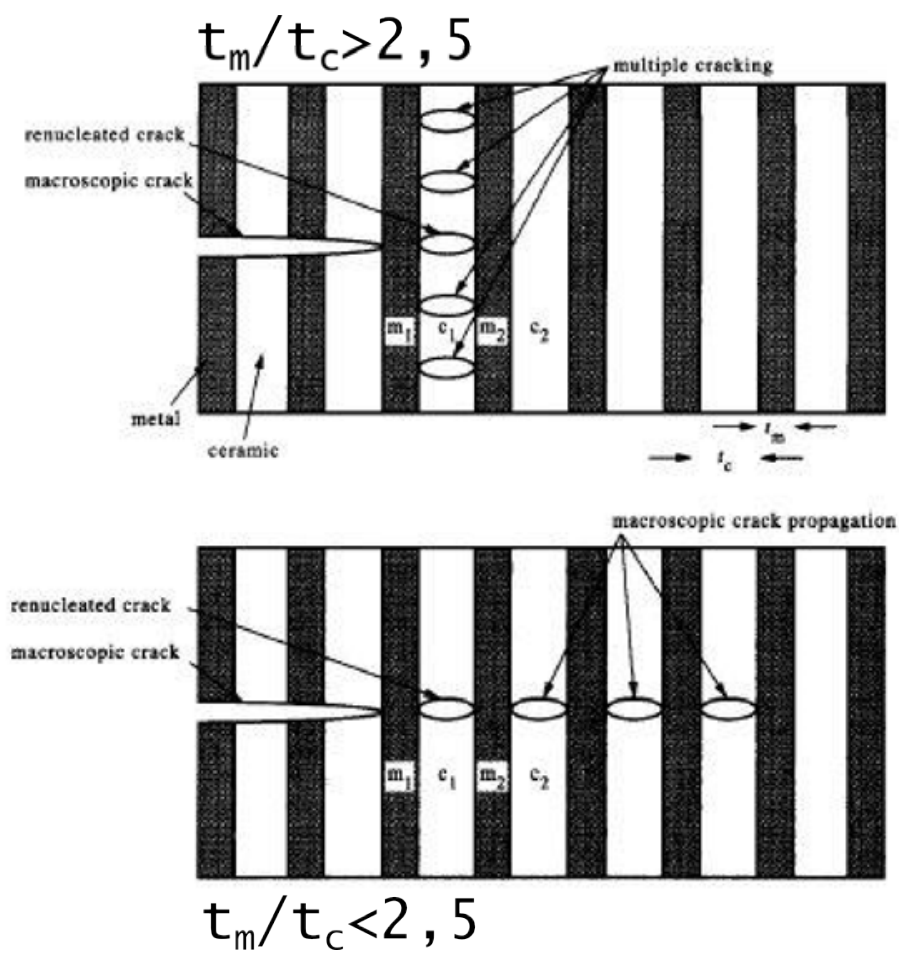

Abbildung 5.5: Schematische Darstellung der Rissmechanismen in metallisch/keramischen Multilagen aus [Hua95]

hinter der Rissspitze nicht berücksichtigt ist. So kann eine plastische Verhärtung der Metallschicht während der Verformung die Rissbrückenbildung unterdrücken [Cao91]. Dieser Effekt wird in dem Modell nicht berücksichtigt.

Bruchversuche an Multilagenstrukturen bestehend aus kollumnaren $\mathrm{Cu}$-Schichten und feinkörnigen Nb- oder Zr-Schichten zeigen eindeutig, dass sowohl der Bruchmechanismus als auch die Duktilität der Multilage stark von der $\mathrm{Cu}$-Schichtdicke abhängt [Zha11]. In der in Abbildung 5.6 dargestellten Geometrie verringert sich für dünnere Kupferschichten aufgrund der längenskalenabhängigen Duktilität des Kupfers Mis03, Mis07, Zhu08, der längenskalenabhängigen Fließspannung und den Einschränkungen der plastischen Zone aufgrund der Schichtdicke (vgl. Abschnitt 2.1.1) die Möglichkeit die Rissspitze abzurunden. Dünnere Kupferschichten sind somit weniger widerstandsfähig gegenüber dem Risswachstum und ein kontinuierliches Risswachstum über die Grenzflächen hinweg wird beobachtet [Zha11].

Wird die kritische Bruchzähigkeit der Metallschicht erreicht und versagt diese, wird der Riss aus der X-Schicht hin in die Metallschicht auch bei dickeren Metallschichten fortgesetzt.

Sowohl für die $100 \mathrm{~nm}$ - als auch für die $50 \mathrm{~nm}$-dicken Ti-Schichten kann die Renukleation des Risses in der Keramikschicht sowie die noch intakten Ti-Schichten direkt beobachtet werden. Für den Fall der 10 nm-dünnen Ti-Schichten werden in den Experimenten keine Rissbrücken beobachtet. Es kann jedoch nicht mit Sicherheit aus- 


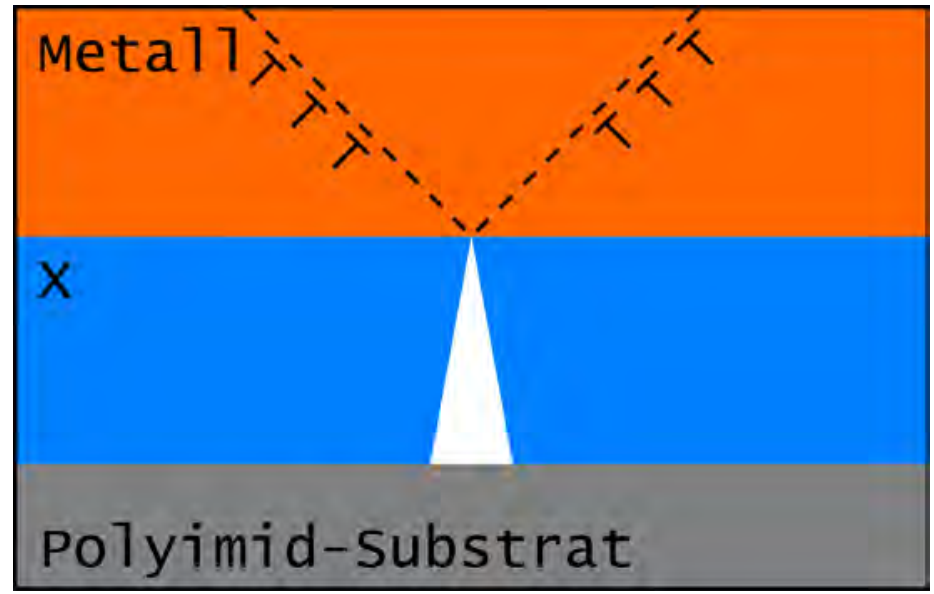

Abbildung 5.6: Schematische Darstellung des Versagensmodell nach Zha11]

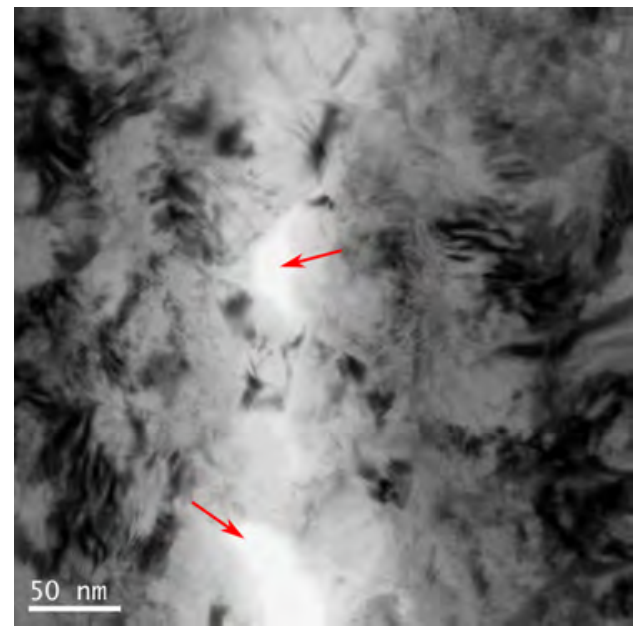

(a) BF

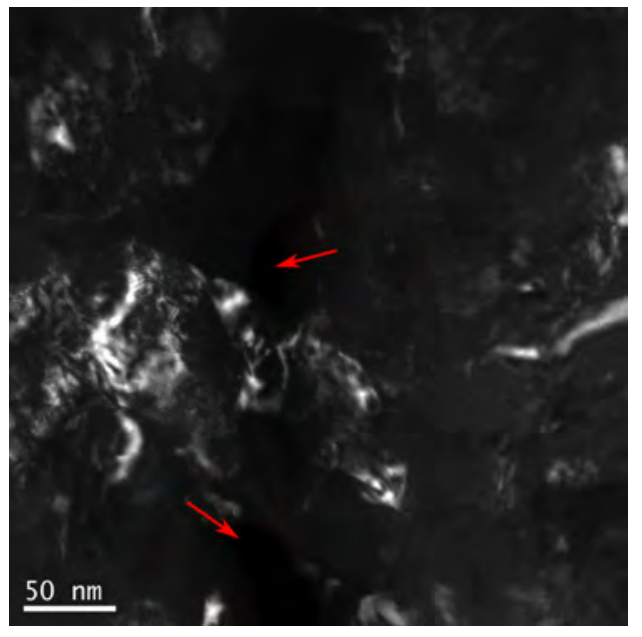

(b) DF

Abbildung 5.7: Hell- und Dunkelfeldaufnahme der Rissbrücken in der Ti-Einzelschicht

geschlossen werden, dass während des Bruchversuchs Rissbrücken in den Ti-Schichten gebildet wurden, die aufgrund des katastrophalen Versagens der Lamelle und der geringen Schichtdicke der Ti-Schichten nicht sichtbar gemacht werden konnten.

Es erscheint wahrscheinlicher, dass aufgrund der verringerten Duktilität und den stärkeren Einschränkungen der plastischen Zone im Vergleich zu den dickeren Ti-Schichten die Rissbrückenausbildung unterdrückt ist Hsi94, Zhu04, Bud04, Kum10, (vgl. Abschnitt 2.1.1).

Weiterhin wird durch die höhere Festigkeit der 10 nm-dicken Ti-Schicht eine höhere aufgebrachte Spannung benötigt, um einen Riss zu bilden bzw. wachsen zu lassen. Mit höheren Spannungen ist die Möglichkeit eines Spaltbruches größer.

Die Mikrostruktur der Metallschichten kann einen entscheidenden Einfluss auf die Wahl der beiden bereits geschilderten Rissmechanismen haben. Obwohl nach den oben vorgestellten Modellen Ti/TiN-Multilagen aufgrund des Schichtdickenverhältnisses über 
den Rissmechanismus der Renukleation von Rissen in den Keramikschichten versagen sollten, wird experimentell das Versagen über die Rissbildung in der Ti-Metallschicht beobachtet Kum10]. Dieses veränderte Verhalten erklärt sich durch die Mikrostruktur der Ti-Schichten, die kollumnare Körner über die gesamte Schichtdicke $(850 \mathrm{~nm}) \mathrm{mit}$ einem mittleren Durchmesser von etwa $25 \mathrm{~nm}$ aufweisen [Kum10]. Diese kollumnare Kornstruktur ermöglicht die Rissbildung entlang der Korngrenzen, die senkrecht zu den Grenzflächen - also in Rissausbreitungsrichtung - orientiert sind.

Ein Risswachstum entlang der Korngrenzen kollumnarer Körner wie in Kum10 kann in den Bruchversuchen senkrecht zur Wachstumsrichtung und damit parallel zu den Korngrenzen der kollumnaren Ti-Körner nicht eindeutig beobachtet werden. Dunkelfeldaufnahmen der Rissbrücken in der Ti-Einzelschicht, die eine ähnliche Mikrostruktur besitzen wie die dickeren Ti-Schichten in den Multilagen, bestätigen, dass die Rissbrücken in einem einzelnen Korn ausgebildet werden (vgl. Abbildung 5.7). Auch die HRTEM-Aufnahmen der Rissbrücken (vgl. Abbildung 4.46) in den Ti-Schichten der Multilagen zeigen eine vergleichbare Situation. Obwohl auch die hier untersuchten TiSchichten ein kollumnares Wachstum zeigen, belegen die Experimente, dass die Korngrenzen nicht die schwächsten Stellen in den Multilagen sind.

\subsubsection{Plastische Zone}

Bezüglich der plastischen Zonen werden im Folgenden zwei Gesichtspunkte diskutiert. Zum einen kann die Größe der plastischen Zone, die in den in-situ TEM-Bruchversuchen anhand der Versetzungsaktivitäten direkt beobachtet wird, analysiert und zum anderen kann die Form der plastischen Zone in den Bruchgeometrien im Vergleich mit der Literatur diskutiert werden.

In Abbildung 5.8 ist für die Ti100/ $\mathrm{ZrO}_{2} 100$-Proben die plastische Zone in der ersten Aufnahme einer Bildserie des jeweiligen Bruchversuchs dargestellt. Die roten Kreise markieren alle Positionen, an denen während des Risswachstums plastische Ereignisse registriert werden, die als Maß für die Größe und auch für die Form der plastischen Zone dienen.

Für den Fall der Bruchversuche entlang der Grenzflächen in Abbildung 5.8a können zwar deutlich weniger plastische Ereignisse beobachtet werden, jedoch zeigt sich hier, dass die plastische Zone in Richtung der Rissausbreitungsrichtung größer zu sein scheint als senkrecht dazu. Diese Asymmetrie kann auch in FEM-Simulationen der plastischen Zone in duktil/spröden Multilagen in der Literatur gefunden werden Cao91, Jha98. Abbildung 5.8b zeigt die plastischen Ereignisse in einer Ti100/Z $\mathrm{ZrO}_{2} 100$-Lamelle, die senkrecht zu ihren Grenzflächen getestet wird. Hier zeigt sich im Vergleich zu Abbildung 5.8a, dass sich die plastischen Ereignisse deutlich homogener verteilen. Es können Positionen mit Versetzungsaktivitäten gefunden werden, die etwa $400 \mathrm{~nm}$ senkrecht zur 


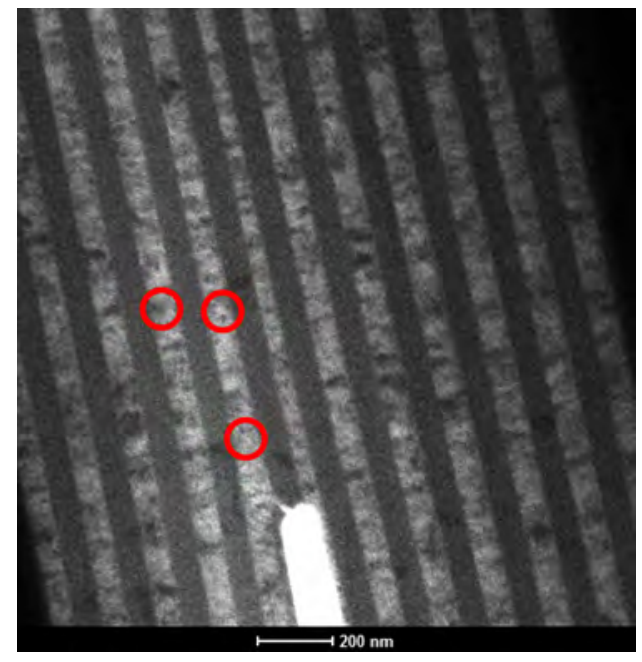

(a) entlang der Grenzflächen

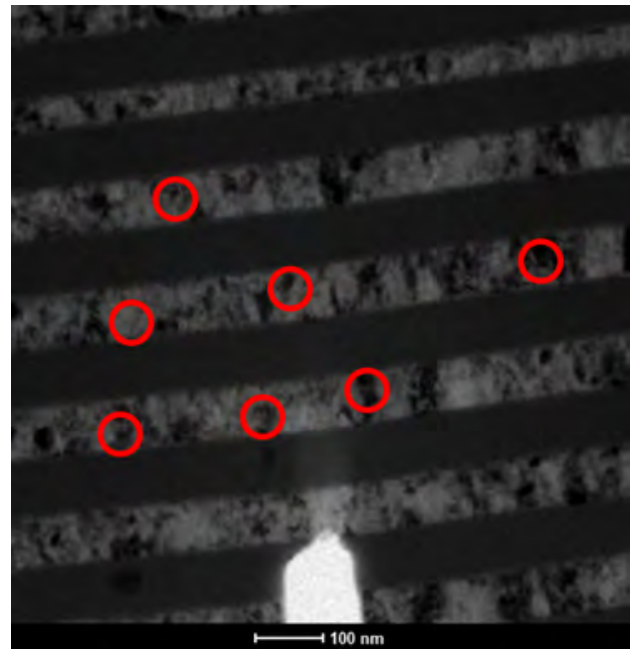

(b) senkrecht zu den Grenzflächen

Abbildung 5.8: Plastische Zone in den Multilagen

Rissausbreitungsrichtung entfernt von dem eigentlichen Riss liegen. In Richtung der Rissausbreitung ist auch hier die plastische Zone größer. Der hier ermittelte Wert liegt bei etwa $600 \mathrm{~nm}$.

Neben den elastischen FEM-Simulationen im Ergebnisteil dieser Dissertation sei eine zusätzlich durchgeführte Simulation erwähnt, die ein perfekt elastisch-plastisches Verhalten der Materialien annimmt. In Abbildung 5.9 sind zwei Aufnahmen dieser Simulation in der Geometrie der Rissausbreitung entlang der Grenzflächen gezeigt. Im Vergleich zu der Form der plastischen Zone der FEM-Simulation in Abbildung 5.9b ist eindeutig festzuhalten, dass in den Experimenten die plastische Zone senkrecht zu der Rissausbreitung geringer ist als in den Simulationen. Es werden nur selten Ereignisse beobachtet, die mehr als eine Ti-Schicht von der Rissspitze entfernt liegen. Dem hingegen erstreckt sich die plastische Zone in Richtung des Risses über mehrere hundert Nanometer (etwa $500 \mathrm{~nm}$ ) im Falle der Ti100/ZrO 2 100-Proben.

Aufgrund der FEM-Simulation in Abbildung 5.9 ist ersichtlich, dass alle Ti-Schichten unter dem Einfluss einer nicht zu vernachlässigenden Spannung stehen, die mindestens etwa der Hälfte der Fließspannung des Titans entspricht. Obwohl die Spannungen in der Nähe der Si-Säulen die Fließspannung des Titans nicht erreichen, ist jedoch mit einer Beeinträchtigung der Berechnung der Größen der plastischen Zonen über Gleichung (2.4) zu rechnen. Die Berechnung der plastischen Zone basiert auf der linearelastischen Bruchmechanik, die verlangt, dass die plastische Zone wesentlich kleiner als die Dimensionen der Probe ist. Diese Voraussetzung ist für den hier vorliegenden Fall nicht gegeben, sodass die berechneten Größen der plastischen Zone nicht uneingeschränkt vertrauenswürdig sind. Für die Ti100/ $\mathrm{ZrO}_{2} 100$-Proben beträgt die gemessene plastische Zone im TEM etwa die Hälfte der gesamten Lamelle, sodass sich für diese Proben die größten Abweichungen zu der berechneten Größe der plastischen Zone 


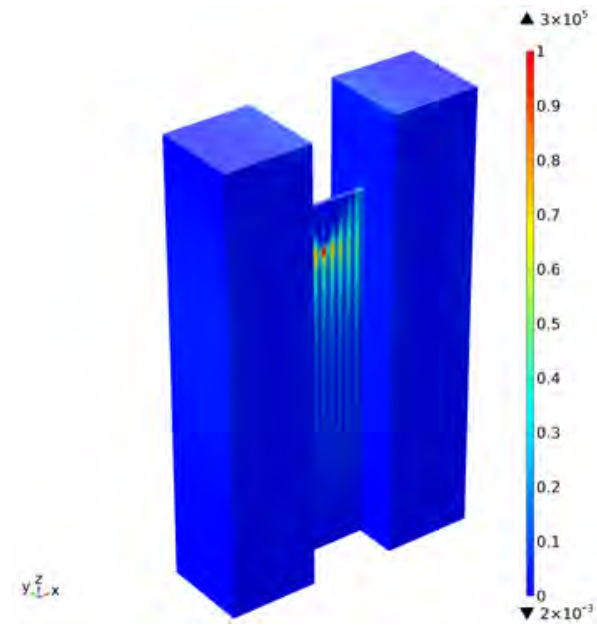

(a)

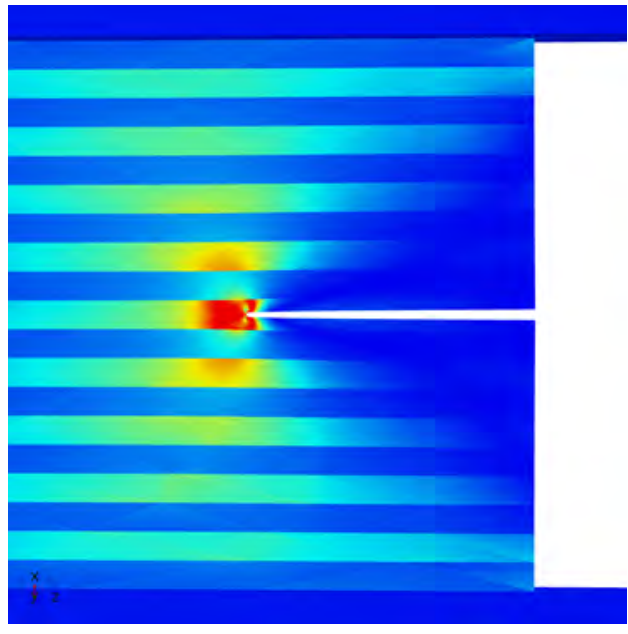

(b)

Abbildung 5.9: Spannungsverteilung der symmetrischen Simulation mit $100 \mathrm{~nm}$ Auslenkung der SiSäulen mit plastischer Verformung der Schichten. Farbskala ist identisch für beide Abbildungen und normiert mit der jeweiligen Fließspannung des Materials $\left(\sigma / \sigma_{y s}\right)$

\begin{tabular}{lccc|ccc} 
& \multicolumn{3}{c}{ entlang der Grenzflächen } & \multicolumn{3}{c}{ senkrecht zu den Grenzflächen } \\
$\begin{array}{l}\mathrm{Ti} / \mathrm{ZrO}_{2}- \\
\text { Probe }\end{array}$ & $r_{p, T E M}$ & $K_{I Q}$ & $r_{p, K}$ & $r_{p, T E M}$ & $K_{I Q}$ & $r_{p, K}$ \\
\hline $100 / 100$ & $492(20)$ & $1,99(20)$ & 219 & $596(10)$ & $3,63(24)$ & 728 \\
$50 / 100$ & $307(25)$ & $1,07(16)$ & 101 & $320(7)$ & $1,44(22)$ & 183 \\
$10 / 100$ & $144(19)$ & $2,27(16)$ & 208 & $71(12)$ & $2,66(656)$ & 286
\end{tabular}

Tabelle 5.2: Plastische Zonen gemessen im TEM und berechnet aus den Bruchzähigkeiten mit Gleichung (2.4)

zeigen. Mit kleinerer Ti-Schichtdicke und damit auch kleinerer plastischer Zone ist zu erwarten, dass die Werte der berechneten plastischen Zone belastbarer werden. Die in Tabelle 5.2 berechneten Werte sind unter diesem Gesichtspunkt zu bewerten.

Auch für die Einzelschichtbruchversuche oder die Bruchversuche in einer Gasatmosphäre kann im Allgemeinen keine Übereinstimmung zwischen den berechneten und gemessenen plastischen Zonen gefunden werden, da auch hier die gemessenen plastischen Zonen etwa die Hälfte der gesamten Lamellenbreite überspannen.

Die gemessenen Größen der plastischen Zone folgen einem klaren Verlauf in Abhängigkeit der Ti-Schichtdicke. Es werden kleinere plastische Zonen in den Multilagen mit dünneren Ti-Schichtdicken gefunden, wie es aufgrund der Einschränkungen der plastischen Zone durch die verringerte Längenskala zu erwarten ist (vgl. Abschnitt 2.1.1). Mit steigender Schichtdicke vergrößert sich die plastische Zone, da die Fließspannung der Ti-Schichten abnimmt (vgl. Abbildung 2.8). Im Vergleich zu der Größe der plastischen Zone in den Ti-Einzelschichten ist hier festzuhalten, dass die gemessenen plastischen 
Zonen vergleichbar groß sind, was aufgrund der ähnlichen Fließspannungen die Erwartungen erfüllt. Es zeigt sich somit, dass in den untersuchten Lamellen die Größe der plastischen Zone im Wesentlichen durch die Fließspannungen der duktilen Ti-Schichten bestimmt wird.

Zusammenfassend muss hier festgehalten werden, dass trotz des klaren Verlaufs, die Bestimmung der plastischen Zone in den Ti10/Z $\mathrm{rO}_{2}$ 100-Proben deutlich schwieriger und teilweise sogar nicht möglich ist. Einen Kontrastwechsel in den nur 10 nm-dünnen Ti-Schichten zu beobachten, ist nur bei relativ hohen Vergrößerungen im TEM möglich. Eine höhere Vergrößerung bringt die Unsicherheit mit sich, dass eventuell nicht alle Kontrastwechsel noch im Bildausschnitt liegen. Das bedeutet, dass die Bestimmung der Größen der plastischen Zonen anhand der TEM-Aufnahmen als untere Abschätzung zu interpretieren ist.

Im Fall der $\mathrm{Ti} 50 / \mathrm{ZrO}_{2} 100$ - und $\mathrm{Ti} 100 / \mathrm{ZrO}_{2} 100$-Proben ist die Beobachtung der plastischen Ereignisse nicht als limitierender Faktor bei der Bestimmung der Größe der plastischen Zone zu betrachten.

\subsubsection{Bruchzähigkeiten und Energiefreisetzungsraten}

Im nachfolgenden Abschnitt wird die kritische Bruchzähigkeit der verschiedenen Versuche in Bezug auf ein längenskalenabhängiges Verhalten im Vergleich mit der Literatur diskutiert. In Abschnitt 2.1.1 ist bereits eine Übersicht der in der Literatur beobachteten Abhängigkeiten der Bruchzähigkeit von Längenskalen verschiedensten Ursprungs erläutert.

In Abbildung 5.10 ist ein Ashby-Diagramm der Bruchzähigkeiten in Abhängigkeit der Festigkeit für verschiedene Materialien gezeigt. Die in dieser Dissertation gemessenen Bruchzähigkeiten sind in das Diagramm eingetragen.

Es muss vorab festgehalten werden, dass hier aufgrund der Abhängigkeit der Bruchzähigkeit von der Probendimension (vgl. Abschnitt 2.1.1) zu erwarten ist, dass alle gemessenen Bruchzähigkeiten kleiner sind als die aufgetragenen Daten in dem AshbyDiagramm. Daher werden hauptsächlich die relativen Lagen der Bruchzähigkeiten von Zirkon und Titan im Experiment und aus der Literatur diskutiert. Es werden jedoch auch Gründe für die geringen Bruchzähigkeiten aufgeführt.

Die berechneten Bruchzähigkeiten der Multilagen werden im Weiteren als Bruchzähigkeiten des Titans interpretiert, da aufgrund der experimentellen Beobachtungen die plastische Verformung, die den größten Anteil zu der Bruchzähigkeit bzw. CTOD liefert, nur in den Ti-Schichten auftritt. Die Berechnung über Gleichung (2.7) erfolgt somit jeweils mit der Fließspannung der Ti-Schicht.

Diese Annahme vernachlässigt den Anteil der elastischen Verformung in den $\mathrm{ZrO}_{2^{-}}$ Schichten, was jedoch aufgrund der relativ ähnlichen Elastizitätsmoduln der $\mathrm{ZrO}_{2}{ }^{-}$ 


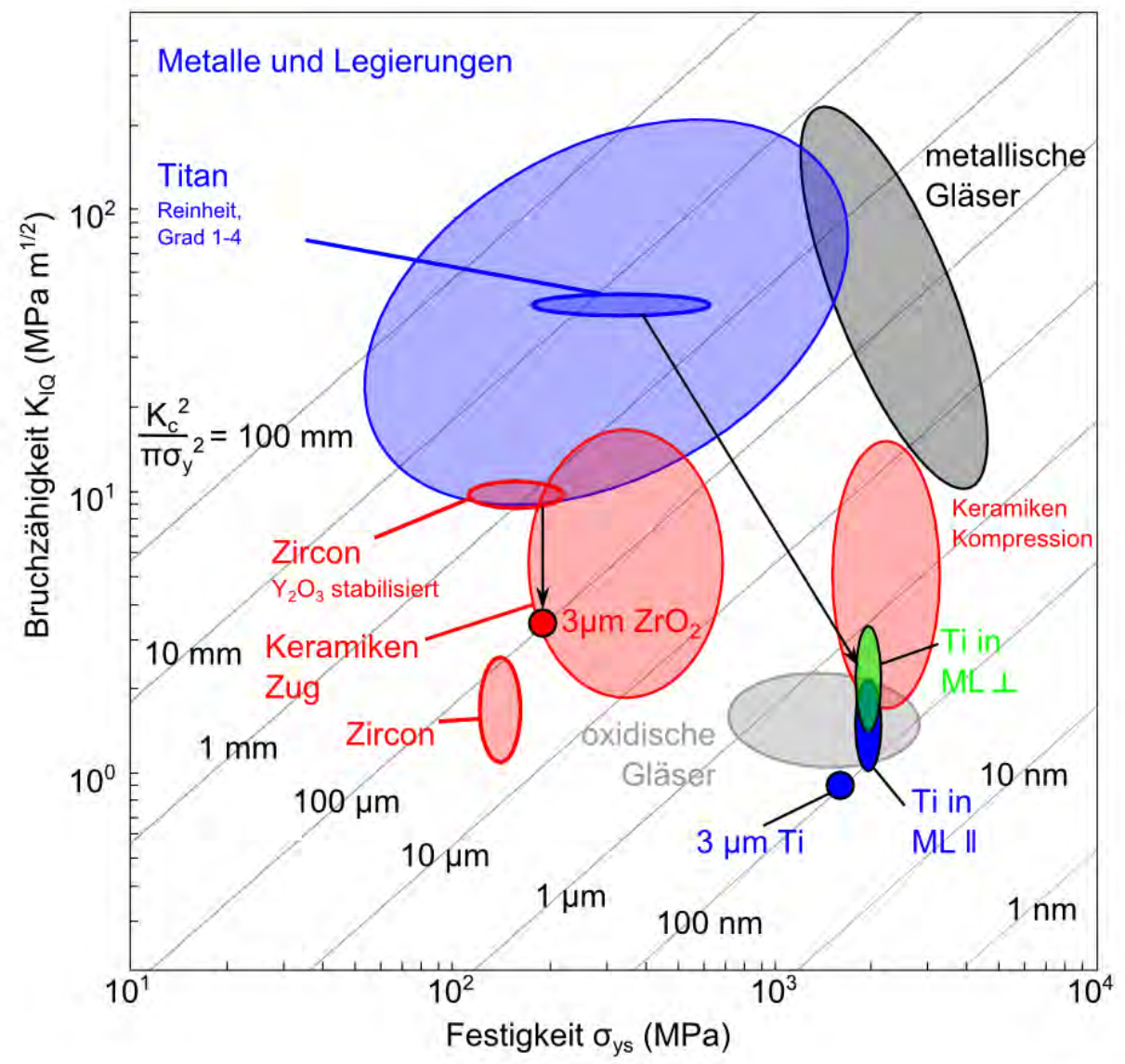

Abbildung 5.10: Ashby-Diagramm nach [Gra13]

bzw. Ti-Schichten (vgl. Tabelle 3.2 nicht stark ins Gewicht fällt. Für die Diskussion des längenskalenabhängigen Verhaltens der Bruchzähigkeiten ist diese Vernachlässigung daher weitergehend irrelevant.

Anhand des Ashby-Diagramms ist eindeutig zu erkennen, dass die gemessenen Bruchzähigkeiten deutlich geringer sind im Vergleich zu denen in der Literatur dokumentierten Daten. Weiterhin sind alle Bruchzähigkeiten der Ti-Schichten (sowohl in den Multilagen als auch in den Einzelschichten) kleiner als die der $\mathrm{ZrO}_{2}$-Einzelschicht, obwohl Zirkon in massiven Proben eine geringere Bruchzähigkeit aufweist als massive Titan-Proben. Das bedeutet, dass sich die relativen Verhältnisse der Bruchzähigkeiten in den dünnen Schichten, die in dieser Dissertation untersucht werden, umgedreht haben.

Der Vergleich der Daten für die amorphe $\mathrm{ZrO}_{2}$-Schicht ist aufgrund der Struktur der vorliegenden Schichten deutlich schwieriger als im Fall des Titans. Die im AshbyDiagramm eingezeichneten Literaturdaten sind an kristallinen Zirkon-Proben gemessen worden, sodass ein Vergleich nur unter Berücksichtigung dieses mikrostrukturellen 
Unterschieds der Proben möglich ist. Es ist anzunehmen, dass durch die amorphe Struktur der $\mathrm{ZrO}_{2}$-Schichten und deren mechanischen Eigenschaften (vgl. Tabelle 5.1. (Hei11b, Kno12]) eine lokale plastische Verformung mittels Scherbändern ermöglicht wird [Spa77, Arg79], die in einer Erhöhung der Bruchzähigkeit resultiert. Dieser Prozess wirkt der Reduzierung der Bruchzähigkeit aufgrund der Miniaturisierung der Proben entgegen.

Im Folgenden werden die Bruchzähigkeiten der Ti-Schichten im Vergleich mit der Literatur und in Abhängigkeit der Schichtdicke diskutiert.

Auch in Aluminium-Schichten in einem ähnlichen Schichtdickenbereich $(100 \mathrm{~nm}$ bis $125 \mathrm{~nm})$ und einer vergleichbaren mittleren Korngröße $(50 \mathrm{~nm})$ wird in in-situ Bruchversuchen im TEM eine kritische Bruchzähigkeit im Bereich von 0,7 $\mathrm{MPa} \mathrm{m}^{1 / 2}$ bis $1,1 \mathrm{MPa} \mathrm{m}^{1 / 2}$ gemessen, die deutlich kleiner ist als die in der Literatur berichteten Werte $\left(15 \mathrm{MPa} \mathrm{m}^{1 / 2}\right.$ bis $30 \mathrm{MPa} \mathrm{m}^{1 / 2}$ Gra13) Kum09. Die geringe Bruchzähigkeit wird über den Übergang zu einen ebenen Spannungszustand und das Fehlen einer Verhärtung des Metalls erklärt.

Die Energiefreisetzungsrate, die bei der Rissbildung ausgehend von einer ideal atomar scharfen Kerbe gemessen werden kann, beträgt theoretisch nur etwa $2 \mathrm{~J} \mathrm{~m}^{-2}$ Kum09. In makroskopischen Bruchexperimenten ist es nicht möglich, eine atomar scharfe Kerbe zu erzeugen. Mit einem größeren Krümmungsradius von etwa $50 \mathrm{~nm}$ wird geschätzt Kum09, dass die Energiefreisetzungsrate um einen Faktor drei größer sein sollte, weil mehr Energie gebraucht wird, um einen Riss von einer abgerundeten Spitze zu bilden. Erst die Miniaturisierung und die damit verbundenen Präparationsmethoden (zum Beispiel FIB) ermöglichen wesentlich geringere Krümmungsradien im Vergleich zu den makroskopischen Experimenten, sodass die schärferen Krümmungsradien dazu beitragen können, dass geringere Bruchzähigkeiten in kleineren Proben gemessen werden.

Die in dieser Dissertation realisierten Krümmungsradien betragen aufgrund der gewählten Präparationsmethode mit dem geringsten Ionenstrahlstrom des FIB weniger als $50 \mathrm{~nm}$, sodass die Beobachtung viel geringerer Bruchzähigkeiten im Vergleich zu massiven Titan-Proben nicht ungewöhnlich erscheint.

Die Mikrostruktur der kristallinen Schichten in einer Multilagen-Struktur oder auch allgemein in Einzelschichten kann die Bruchzähigkeit mit bestimmen. Mit steigender Korngröße steigt auch die Bruchzähigkeit der kristallinen Schicht Mus82, Swa86. In CrN-Kompositmaterialien wird von einem Anstieg der Bruchzähigkeit um einen Faktor zwei berichtet Liu13], wenn die Mikrostruktur von einem kollumnar gewachsenen Material hin zu feinkörnigen Schichten geändert ist.

In-situ TEM-Bruchversuche an Ti/TiN-Multilagen mit konstanten Schichtdicken $\left(d_{\mathrm{Ti}}=850 \mathrm{~nm}\right.$ und $\left.d_{\mathrm{TiN}}=450 \mathrm{~nm}\right)$ zeigen deutlich verringerte Bruchzähigkeiten von etwa $1,45 \mathrm{MPam}^{1 / 2}$ bis 2,45 $\mathrm{MPam}^{1 / 2}$ in der Geometrie der Rissausbreitung 


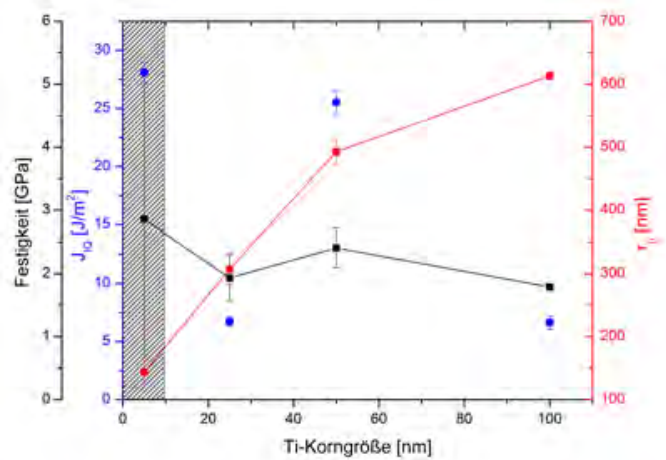

(a) entlang

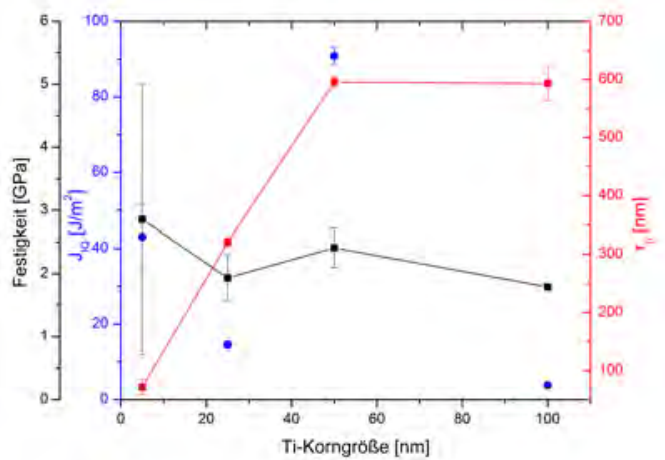

(b) senkrecht

Abbildung 5.11: Energiefreisetzungsrate, Größe der plastischen Zone und Fließspannungen der TiSchichten für die Bruchveruche entlang der Grenzflächen (a) und senkrecht zu den Grenzflächen[(b)

senkrecht zu den Grenzflächen in Abhängigkeit der Lamellendicke (150 nm bis $300 \mathrm{~nm}$ ) in Elektronenstrahlrichtung Kum10. Die verringerten Bruchzähigkeiten stehen in Verbindung mit dem Übergang in den ebenen Spannungszustand.

Zusammenfassend bleibt festzuhalten, dass durch die zunehmenden Einschränkungen der plastischen Zone mit kleinerer Probendimension kleinere Bruchzähigkeiten zu erwarten sind |Var91, Rit93, Was96, Kru04]. Zusätzlich trägt die längenskalenabhängige Fließspannung dazu bei, dass auf größeren Dimensionen mehr Energie dissipiert werden kann, sodass die Bruchzähigkeit mit der Probendimension ansteigt. Auch die Fähigkeit der Rissspitzenabschirmung steigt mit der Schichtdicke, wodurch die Proben im Allgemeinen widerstandsfähiger werden [Cao91,Hwu99. Diese Mechanismen bestätigen, dass die Bruchzähigkeit mit kleinerer Dimension bzw. Schichtdicke verringert wird. Dieser Trend wird auch in den Bruchversuchen beobachtet.

Nachfolgend wird der Verlauf der Energiefreisetzungsraten in Bezug auf die TiKorngröße diskutiert. In Abbildungen 5.11a und 5.11b sind die Energiefreisetzungsraten, die Größe der plastischen Zone und die Festigkeiten aus [Kno12] in Abhängigkeit der Ti-Korngröße für beide realisierten Bruchgeometrien aufgetragen. Zusätzlich sind, zu einer besseren Einordnung, die Ergebnisse der Ti-Einzelschicht senkrecht zu ihrer Wachstumsrichtung gezeigt. Die mittlere in-plane Korngröße der Ti-Einzelschichten wird anhand der TEM-Aufnahmen mit etwa $100 \mathrm{~nm}$ angenommen.

Alle gemessenen Energiefreisetzungsraten sind deutlich geringer als typische Werte für Metalle. Diese großen Abweichungen (etwa $1 \times 10^{5}$ ) sind anhand der Randbedingungen, die durch die Geometrie der Lamellen als auch der Multilagen hervorgerufen werden, zu erklären. Dieser Effekt ist bereits diskutiert worden.

Anhand der Abbildungen ist eindeutig zu erkennen, dass sowohl die Größe der plastischen Zone als auch die Festigkeit eine Abhängigkeit von der Ti-Korngröße zeigt. Die Energiefreisetzungsrate folgt diesem klaren Verlauf der Größe der plastischen Zone 
nicht. Eine Abweichung ist sowohl bei sehr kleinen als auch bei großen Ti-Körnern zu beobachten. Die Abweichung bei kleinen Ti-Korngrößen ist im Fall der Bruchversuche entlang der Grenzflächen durch einen veränderten Bruchmechanismus zu erklären (schraffierter Bereich in Abbildung 5.11a). Für die geringsten Ti-Schichtdicken und damit auch Korngrößen kann eine Rissausbreitung entlang der amorph/kristallinen Grenzflächen beobachtet werden. Das bedeutet, dass diese Ti-Schichten im Vergleich zu den größeren Ti-Schichten einen weniger entscheidenden Einfluss auf das Bruchverhalten haben. Die Festigkeit der 10 nm-dicken Ti-Schichten überschreitet die Festigkeit der Grenzfläche (bestimmt anhand der 45-Säulen aus [Kno12]), sodass in den Ti10/ $\mathrm{ZrO}_{2}$ 100-Proben die Grenzflächen als mikrostrukturell schwächste Komponenten angesehen werden und der Riss daher entlang der Grenzflächen verläuft.

Die geringe Energiefreisetzungsrate bei großen Ti-Körnern erklärt sich durch Veränderungen in den Randbedingungen der Rissausbreitung und der plastischen Zone. In den Multilagen kann der Riss aufgrund der Mikrostruktur der Ti-Schicht (kollumnare Körner) und der aufgezwungenen Randbedingungen durch die benachbarten $\mathrm{ZrO}_{2}{ }^{-}$ Schichten nicht entlang der mikrostrukturell schwächsten Richtung wachsen, sodass der Riss Körner durchqueren muss anstatt entlang von Korngrenzen zu wachsen. In der Ti-Einzelschicht sind keine Randbedingungen wie in den Multilagen zu erwarten, sodass der Riss hier die Ausbreitungsrichtung entlang der schwächsten Richtung wählen kann. Aufgrund des Risspfades (vgl. Abbildung 4.13a) ist eindeutig zu erkennen, dass der Riss mehrfach seine Richtung ändert, um entlang der schwächsten Richtung zu wachsen.

Da die Energiefreisetzungsrate im Wesentlichen durch die plastische Verformung in den Metallschichten bestimmt wird, ist ein Anstieg der Werte mit steigender Größe der plastischen Zone und zusätzlich durch die geringere Festigkeit der Ti-Schichten mit steigender Schichtdicke plausibel und deckt sich mit den theoretischen Erwartungen. Diese Beobachtung gilt sowohl für die Ti50/ZrO 2100 - als auch für die Ti100/ZrO 2 100Proben in beiden Bruchgeometrien.

Die deutlich erhöhte Energiefreisetzungsrate der Ti10/Z $\mathrm{rO}_{2}$ 100-Proben in der Geometrie der Rissausbreitung senkrecht zu den Grenzflächen kann zum einen aufgrund des katastrophalen Versagens nur mit einer größeren Unsicherheit bestimmt werden. Zum anderen wurde bereits erwähnt, dass mit kleinerer Ti-Schichtdicke die Duktilität des Titans verloren geht, sodass dieses versprödet. Mit geringerer Plastizität des Titans ist hier anzunehmen, dass das Bruchverhalten sich dem Verhalten der $\mathrm{ZrO}_{2}$-Einzelschicht annähert, sodass ein Anstieg in der Energiefreisetzungsrate im Vergleich zu den Werten der $\mathrm{Ti} 50 / \mathrm{ZrO}_{2} 100$-Probe $\mathrm{zu}$ erwarten ist. 


\subsection{Bruchverhalten in einer Gasatmosphäre}

Das Bruchverhalten in einer $\mathrm{H}_{2}$-Gasatmosphäre unterscheidet sich von dem Verhalten ohne Gas in der Art, dass es in der Geometrie der Rissausbreitung entlang der Grenzflächen zum Versagen der $\mathrm{ZrO}_{2}$-Schichten kommt. In der Geometrie senkrecht zu den Grenzflächen ist keine signifikante Veränderung im Bruchverhalten zu beobachten. Für eine $\mathrm{H}_{2} \mathrm{O}$-Atmosphäre wird kein verändertes Bruchverhalten festgestellt.

Eine Gasatmosphäre kann verschiedenste Einflüsse auf die Materialien haben. Einer der bekanntesten Mechanismen in Verbindung mit einer Gasatmosphäre ist die Spannungsrisskorrosion. Spannungsrisskorrosion kann oft zu einem unerwarteten und katastrophalen Versagen der Probe führen. Durch den Einfluss der Gasatmosphäre wird die Bruchzähigkeit herabgesetzt und das Material versagt somit bei niedrigeren aufgebrachten Spannungen.

Welches Gas einen Einfluss auf des jeweilige Material hat, ist nur schwer vorherzusagen. Aus der Literatur ist bekannt, dass zum Beispiel Keramiken mit einem hohen ionischen Bindungsanteil anfällig für die Spannungsrisskorrosion gegenüber Wasserdampf sind Che05b. Auch Zirkon fällt in diese Kategorie und es besteht somit die Möglichkeit eines Einflusses der gewählten Gasatmosphäre auf das Bruchverhalten.

Während der Wasserdampf-Experimente ist es möglich, dass der Elektronenstrahl im TEM die Wassermoleküle aufspaltet, sodass neben Wassermolekülen auch Wasser- und Sauerstoff in der Gasatmosphäre vorliegen [Wag12] und somit die Einlagerung von Sauer- als auch Wasserstoff nicht ausgeschlossen werden kann. Für Titan ist bekannt, dass Sauerstoff das Material dramatisch verhärten kann [Lüt00, Yu15]. In Verbindung mit den chemischen Analysen aus Kno12 (SIMS-Messungen) und den EELS-Daten aus dieser Dissertation, die ein Sauerstoffsignal in den deponierten Titanschichten (in den Multilagen und auch in den Einzelschichten) zeigen, ist davon auszugehen, dass durch die Sauerstoffverunreinigung der Ti-Schichten die Festigkeit entscheidend angehoben wird. Titanoxid-Verbindungen können aufgrund der Feinstruktur der Ti L-Kante charakterisiert werden Lea82. Allein TiO kann nicht von reinem Ti im EELS unterschieden werden [Sto07, sodass nicht mit Sicherheit festgestellt werden kann, ob die Ti-Schichten aufgrund der geringen Dicke nicht bereits durch die Umgebung oxidiert sind, bevor sie einer Gasatmosphäre ausgesetzt werden und somit die Einlagerung des Gases verlangsamt oder sogar komplett unterdrückt wird.

Da jedoch keine Veränderung im Bruchverhalten in einer Wasserdampf-Atmosphäre zu beobachten ist, ist nicht davon auszugehen, dass durch eine weitere Verhärtung des Titans die $\mathrm{ZrO}_{2}$-Schichten oder die Grenzflächen die schwächsten Komponenten werden und versagen. Die EELS-Daten zeigen keine Veränderungen in der Feinstruktur der Ti L-Kanten, sodass eine signifikante Veränderung des Oxidationszustands ausgeschlossen werden kann. Die Verschiebung der Peak-Positionen zu kleineren Energieverlusten 
könnte auf eine Reduktion des Titans durch den Einfluss des Wasserdampfes hindeuten, was aufgrund der vorhandenen Daten jedoch nicht eindeutig bewiesen werden kann und daher lediglich als eine Möglichkeit anzusehen ist.

Der Einfluss einer Wasserstoffatmosphäre auf das Bruchverhalten ergibt sich im Wesentlichen durch seine versprödende Wirkung auf die beteiligten Materialien. Es wird von verschiedenen Mechanismen der Wasserstoffversprödung in der Literatur berichtet, die hier jedoch nicht im Detail diskutiert werden Bar09.

Im Fall des Titans ist aus der Literatur bekannt, dass sich unter dem Einfluss einer Wasserstoffatmosphäre Titanhybrid-Verbindungen bilden können Bir94. Neben der Bildung von Hybridphasen ist weiterhin berichtet worden, dass Wasserstoff die Kornstruktur des Titans hin zu kleineren Korngrößen verändern kann Yok02. Kleinere Korngrößen ermöglichen bevorzugt einen spröden Rissmechanismus und damit geringere Bruchzähigkeiten Mus82, Ovi07, wobei eine signifikante Verringerung der Korngröße in den Experimenten dieser Dissertation nicht beobachtet werden konnte.

Im Bezug auf die mechanischen Eigenschaften hat eine Wasserstoffatmosphäre entscheidenden Einfluss. So wird in der Literatur berichtet, dass durch weitere Zugabe von Wasserstoff die Fließspannung und die Bruchspannung in mit wasserstoffbeladenen Titan-Proben verringert werden kann [Tet01].

Die Wasserstoffversprödung zeigt sich im Fall des Titans am deutlichsten in einer Verringerung der Duktilität im Vergleich zum unbeeinflussten Material [Don00,Dol15]. Für Titan ist jedoch zu berücksichtigen, dass sich an einer Oberfläche, die der Umgebung ungeschützt ausgesetzt wird, eine natürliche Oxidschicht von wenigen Nanometern bildet Lu00]. Diese Oxidschicht fungiert als Barriere für die Einlagerung des Wasserstoffs in das Titan, wenn sie geschlossen ist Don00. Die $\mathrm{Ti} / \mathrm{ZrO}_{2}$-Grenzflächen hingegen könnten eine Einlagerung des Wasserstoffs in das Titan ermöglichen. In der $\alpha$-Phase ist Titan ohne Verunreinigungen sehr widerstandsfähig gegenüber der Wasserstoffeinlagerung. Aufgrund der Annahme, dass die vorliegenden Ti-Schichten nicht komplett frei von Verunreinigungen sind, ist zu erwarten, dass theoretisch Wasserstoff in den Schichten eingelagert werden kann Don00.

Die geringe Verschiebung der Peak-Positionen der Ti L-Kanten unter der Wasserstoffatmosphäre kann als eine Oxidation des Titans gedeutet werden [Sto07], wobei aufgrund der Wasserstoffatmosphäre eigentlich nicht zu erwarten wäre, dass eine Oxidation auftritt. Es ist jedoch nicht auszuschließen, dass Sauerstoff während des Experiments im Probenraum vorhanden ist.

Wenn Wasserstoff in das Titan eindringen kann, dann können in Abhängigkeit seines Anteils verschiedene Mechanismen auftreten. Für einen geringen Wasserstoffgehalt ist der HELP-Mechanismus (hydrogen enhanced localized plasticity, [Bir94]) bekannt, bei dem durch die Wasserstoffeinlagerung lokal eine erhöhte Versetzungsaktivität auftre- 
ten kann. Steigt der Wasserstoffanteil weiter, so kann es zur Bildung der Titanhybride kommen. In der Literatur wird berichtet, dass ab einem Wasserstoffanteil von etwa 30 ppm Titanhybridphase gebildet werden [Don00]. Der Plateaudruck beträgt etwa $1 \times 10^{-15}$ mbar Kür12]. Die mechanischen Eigenschaften der Titanhybride zeigen geringere Elastizitätsmoduln und Härten, die beide abhängig vom Wasserstoffgehalt sind [Set04]. Die Härte sinkt mit der weiteren Zugabe von Wasserstoff.

Für Zirkonlegierungen ist eine geringe Löslichkeit des Wasserstoffs bekannt, die dazu führt, dass Hybridverbindungen gebildet werden [Var00]. Die mechanischen Eigenschaften werden durch den Wasserstoff herabgesetzt. Weiterhin zeigen die Zirkonhybride ein sprödes Verhalten und eine signifikante Reduzierung der Bruchzähigkeit [Col66]. Für Zirkon wird außerdem berichtet, dass die Hybridbildung einen Rissmechanismus ermöglicht, in dem durch die Spannungen in der Nähe der Rissspitze der Wasserstoff aufgrund des Spannungsgradienten hin zur Rissspitze diffundiert und dort Hybride bildet. Wenn die aufgebrachte Spannung hinreichend hoch ist, versagt das Zirkonhybrid und ein Mikroriss entsteht, der im Verlauf weiter wachsen kann bis die Probe versagt $\operatorname{Var} 02$.

Auch ein Beispiel aus der Medizintechnik zeigt, dass die tetragonale Form des Zirkonoxid, welche im Allgemeinen als die stabilste und risswiderstandsfähigste Phase angesehen wird, in der jeweiligen Anwendung eine Phasenumwandlung zur monoklinen Phase durch die Wechselwirkung mit Wasserstoff vollzieht. Durch die Expansion während dieser Umwandlung kann es zum Versagen des Materials kommen Guo04, Che09, You14. Zusammenfassend ist zu erwarten, dass aufgrund der qualitativen Beobachtungen der in-situ Bruchversuche in einer Wasserstoff-Gasatmosphäre die $\mathrm{ZrO}_{2}$-Schichten so stark durch den Wasserstoff in ihren mechanischen Eigenschaften und Bruchverhalten geschwächt werden, dass sie die schwächste Komponente in den Multilagen sind. Eine Aussage, ob und wie stark die Ti-Schichten durch den Wasserstoff verspröden, erlauben die vorhandenen Daten dieser Dissertation nicht. Jedoch bleibt eindeutig festzuhalten, dass eine Gasatmosphäre das Bruchverhalten entscheidend beeinflusst. 


\section{Zusammenfassung}

Diese Arbeit befasst sich mit einer umfangreichen Charakterisierung des Bruchverhaltens von amorph/kristallinen Multilagen mit Schichtdicken kleiner als $100 \mathrm{~nm}$.

Da das Bruchverhalten auf der Nanoskala bisher nur wenig untersucht wurde und daher noch nicht umfassend verstanden ist, wird im Rahmen der Dissertation eine neue Methodik entwickelt, die diese Untersuchungen ermöglicht. Der realisierte Bruchversuch wird in-situ in einem Transmissionselektronenmikroskop durchgeführt. Dieser Aufbau zeichnet sich durch ein kontrolliertes Risswachstum aus, sodass neben der sehr guten räumlichen Auflösung, weitergehende Analysen bezüglich des Risspfades oder der mikrostrukturellen Prozesse durchgeführt werden können. Dabei zeigt sich, dass diese Art der Bruchversuche besonders flexibel im Bezug auf die Auswahl der zu untersuchenden Probengeometrien sowie -materialien ist. Mit der Beobachtung der mikrostrukturellen Prozesse während des Risswachstums wird auf die Größe der plastischen Zone geschlossen. Damit sind die hier entwickelten in-situ Bruchversuche eines der wenigen Beispiele aus der Forschung im Bereich der Bruchmechanik, die eine wesentliche Kenngröße des Bruchverhaltens direkt sichtbar und messbar machen.

Für die Bruchversuche der Ti-Einzelschichten bleibt festzuhalten, dass sie die erwarteten Merkmale eines duktilen Bruchverhaltens zeigen, wie es auch für andere duktile Materialien beobachtet wird. Die Versetzungsaktivität in ausgewählten Körnern in der Nähe der Rissspitze weist eindeutig auf eine ausgeprägte Plastizität hin, die das Risswachstum begleitet. Auch bezüglich des gewählten Risspfades werden die Erwartungen erfüllt. Der gekrümmte Risspfad erklärt sich durch die verringerten Randbedingungen im Vergleich zu den Multilagen, sodass sich der Riss entlang der schwächsten Richtung ausbreitet.

Obwohl die Bruchzähigkeiten der Ti-Einzelschichten typische Größen eines spröden Materials zeigen, ist durch die in-situ Bruchversuche eindeutig ein duktiles Bruchverhalten nachgewiesen worden. Diese Erkenntnis zeigt nochmals auf, welcher Mehrgewinn 
an Informationen durch die in-situ Bruchversuche erreicht wird. Nur mit der Analyse der mikrostrukturellen Prozesse und des qualitativen Bruchverhaltens während des Risswachstums zusammen mit den quantitativen Daten der Bruchzähigkeit kann das Bruchverhalten eines Materials umfassend und aussagekräftig beschrieben werden.

Für die amorphe $\mathrm{ZrO}_{2}$-Einzelschicht wird eine Bruchzähigkeit gemessen, die vergleichbar mit Bruchzähigkeiten typisch spröder Materialien ist. Das qualitative Bruchverhalten ist deutlich komplexer, da es zu Beginn sowohl einen duktilen Charakter als auch letztlich das spröde Versagen zeigt, sodass kein klarer Bruchmechanismus angegeben werden kann. Das Bruchverhalten amorpher Materialien ist noch nicht hinreichend untersucht, sodass hier weitere Daten notwendig sind, um Mechanismen analysieren und bestätigen zu können.

Für die in-situ Bruchversuche der Multilagen in der Geometrie der Rissausbreitung parallel zu den Grenzflächen wird im Gegensatz zu anderen Beispielen aus der Literatur eine gute Übereinstimmung mit theoretischen Vorhersagen bezüglich der Wahl des Risspfades gefunden. Die Übereinstimmung begründet sich durch die hohe Qualität der untersuchten Multilagen-Proben der vorliegenden Dissertation. Diese ideale Grundlage ermöglicht, dass der Risspfad allein durch Unterschiede in den Materialeigenschaften bestimmt wird und beispielsweise nicht durch Variationen in den Grenzflächeneigenschaften oder der Mikrostruktur. Weiterhin werden Analysen der Mechanismen und Effekte durch die verringerte Längenskala, die einen wichtigen Einfluss auf das Bruchverhalten in einer komplexen Multilagenstruktur hat, durchgeführt. Als entscheidend haben sich die längenskalenabhängige Festigkeit der Ti-Schichten und die Einschränkungen an die Größe der plastischen Zone mit veränderter Ti-Schichtdicke herausgestellt. Da das Bruchverhalten mit verringerter Ti-Schichtdicke immer spröder wird, bleibt festzuhalten, dass die Einschränkungen an die Größe der plastischen Zone die gewichtigere Rolle spielen.

Die Wahl der Risspfade in den Ti-Schichten der Multilagen zeigt, dass sich der Riss aufgrund der kollumnaren Mikrostruktur in Verbindung mit den erzeugten Randbedingungen durch die Multilagengeometrie nicht generell entlang der potentiell schwächsten Richtung ausbreitet, sondern die kollumnaren Körner durchqueren muss. Diese Beschränkung des Risspfades hat direkten Einfluss auf das Bruchverhalten und damit auch auf die Bruchzähigkeit der Materials.

Für die Bruchversuche senkrecht $\mathrm{zu}$ den Grenzflächen zeigt sich für alle TiSchichtdicken das erwartete Verhalten eines Bruchversuchs in vergleichbarer Geometrie. Unabhängig von der Ti-Schichtdicke wird die Rissbrückenbildung in den duktilen TiSchichten in Verbindung mit der Renukleation der Risse in den spröden $\mathrm{ZrO}_{2}$-Schichten als vorherrschender Bruchmechanismus beobachtet. 
Die theoretische Analyse und die direkte Messung der Größe der plastischen Zone bestätigt den erwarteten Verlauf mit sinkender Ti-Schichtdicke und nimmt damit entscheidend Einfluss auf das Bruchverhalten. Weiterhin zeigen die Ergebnisse, dass die Größe der plastischen Zone auch für komplexe Multilagenstrukturen belastbare Analysen liefert, obwohl sie durch die Randbedingungen in der Multilagengeometrie und durch die reduzierte Längenskala beeinflusst wird.

Die quantitativen Analysen der Bruchzähigkeiten der Multilagenproben der verschiedenen realisierten Bruchgeometrien bestätigen die Erwartung, dass die Rissausbreitung senkrecht zu den Grenzflächen schwieriger ist als die parallel zu den Grenzflächen. Es fällt jedoch weiterhin eindeutig auf, dass das Verhalten der Bruchzähigkeit in Abhängigkeit der Ti-Schichtdicke bzw. Korngröße in beiden Geometrien vergleichbar ist. Diese Beobachtung lässt den Schluss zu, dass die Mechanismen, die das Bruchverhalten entscheidend bestimmen, unabhängig von der eigentlichen Geometrie des Bruchversuchs sind. Diese Erkenntnis eröffnet somit einen neuen Ansatz, das Bruchverhalten von Materialien unabhängig von der späteren Anwendung zu beeinflussen. Wenn die bestimmenden Mechanismen ausgemacht und kontrolliert werden können, besteht die Möglichkeit, das Bruchverhalten gezielt einzustellen.

Die Möglichkeit aufgrund der ablaufenden Bruchmechanismen neue komplexe Kompositmaterialien zu entwickeln, stellt somit ein vielversprechendes Konzept für weitere Untersuchungen dar.

Die Experimente in einer Gasatmosphäre in dieser Dissertation liefern die Bestätigung der anfänglichen Idee der Beeinflussung des Bruchverhaltens mit externen Stimuli. Obwohl nicht erreicht werden kann, dass in einer Gasatmosphäre der Risspfad entlang der Grenzflächen der Multilagen verläuft, wird der bestimmende Einfluss einer reaktiven Gasatmosphäre eindeutig aufgezeigt. Auch hier ermöglicht diese Bestätigung einen potentiellen Ansatz, das Bruchverhalten in der gewünschten Art und Weise einzustellen und zu kontrollieren.

Zusammenfassend zeigen die Ergebnisse und Analysen in dieser Dissertation, dass über die Kenntnis der grundlegenden Mechanismen des Bruchverhaltens amorph/kristalliner Multilagen auf der Nanoskala und der Wechselwirkung mit einer Gasatmosphäre, neue Ideen und Methoden entwickelt werden können, einen Riss zu kontrollieren und zu steuern. 


\section{Bruchversuch im SEM}

Zusätzlich zu den in-situ Bruchversuchen im TEM werden Bruchversuche identischer Geometrien im SEM realisiert. Hier ist neben der reinen qualitativen Analyse der Bildserien auch eine quantitative Auswertung der aufgewendeten Kraft möglich. Der in-situ Aufbau im SEM ist im Rahmen einer Diplomarbeit zur Zugverformung von GoldNanodrähten entstanden [Kap11]. Er besteht aus drei Piezo-Aktuatoren, je einen für die Bewegung in einer Raumrichtung. Zusätzlich können über einen MEMS-basierten Kraftsensor (Microelectromechanical systems) quantitativ Kräfte gemessen werden. Die Spitze der Kraftsensoren besteht aus Silicium, die in jede beliebige Form gebracht werden kann. Mit dem FIB wird ein Sensor mit einer keilförmigen Spitze analog zu den Bruchversuchen im TEM versehen. Die Probenpräparation unterscheidet sich für die SEM-Versuche nicht wesentlich von der Präparation für die TEM-Versuche und wird daher nicht weiter beschrieben. Die Geometrie im SEM ist so gestaltet, dass die Spitze bzw. der Kraftsensor ortsfest montiert ist und daher die Probe auf die Spitze zu bewegt wird.

Der Bruchversuch wird hier in der Ausrichtung der Rissbildung senkrecht zu den Grenzflächen durchgeführt. Während die Probe gegen die Spitze gedrückt wird, steigt die Kraft monoton stetig an, wie der verkleinerte Graph in Abbildung A.1c zeigt. Im SEM-Video kann hier zu Beginn der Verformung eine Öffnung der ursprünglichen Kerbe und eine Verbiegung der Si-Säulen beobachtet werden. Die Bildung des Risses findet im grau markierten Bereich des Graphen statt. Die SEM-Aufnahmen zeigen eindeutig die Bildung des Risses und dessen Bewegung durch die Lamelle. In den Daten der aufgebrachten Kraft kann hier im Bereich der Rissbildung keine signifikante Auswirkung der Rissbildung auf die aufgewendete Kraft ausgemacht werden. Es bleibt zu vermuten, dass lediglich die elastische Verbiegung und die dafür notwendige Kraft aufgezeichnet wird. Der Einfluss des Risses auf die tatsächlich gemessene Kraft ist zu klein, um gemessen werden zu können. Auch eine Veränderung in der Steigung der Kraft kann nicht beobachtet werden. Die Risskonfiguration nach dem Versagen der Probe ist vergleich- 


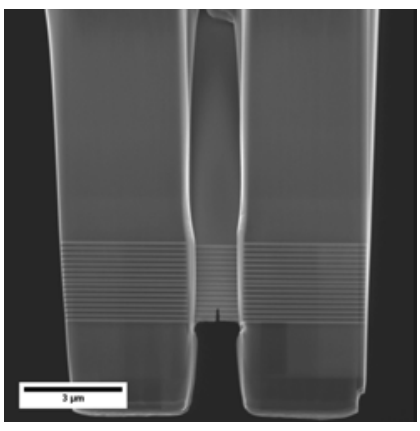

(a)

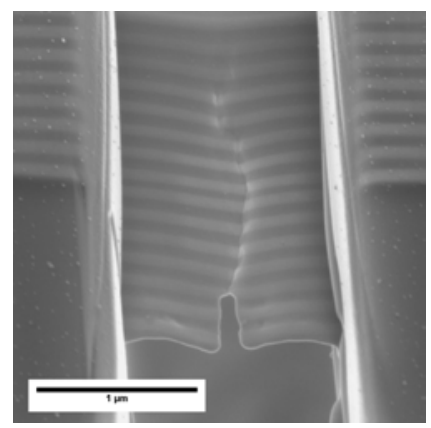

(b)

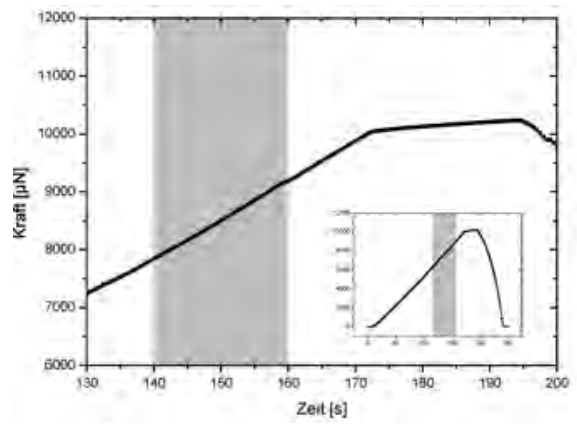

(c)

Abbildung A.1: Quantitativer Bruchversuch im SEM in senkrechter Ausrichtung:(a) SEM-Aufnahme der fertig präparierten Probe; (b)]SEM-Aufnahme nach dem Bruchversuch; (c) Kraftverlauf aufgezeichnet mit dem Kraftsensor, grau markiert der Bereich der Rissbildung/-bewegung, verkleinert dargestellt: Kraftverlauf über den gesamten Bruchversuch

bar zu TEM-Bruchversuchen.

Zusätzlich zu den quantitativen Experimenten werden auch qualitative Bruchversuche durchgeführt. In diesen Fällen wird die Probe gegen eine Wolfram-Spitze gedrückt wie in den Bruchversuchen im TEM. Sie zeigen ein übereinstimmendes Bruchverhalten in der Geometrie für die Rissbildung entlang der Grenzfläche im Vergleich zu den TEMVersuchen entsprechender Ausrichtung und es werden daher hier keine Daten dieser Experimente gezeigt. 


\section{Zugversuche}

Nachfolgend werden Zugversuche an den Ti100/ZrO 100 Proben dargestellt. Diese Zugversuche werden wie auch die bereits beschriebenen Bruchversuche im TEM durchgeführt, deren uniaxiale Zugrichtung senkrecht zu den Grenzflächen der Materialien orientiert ist. Mithilfe eines Einfachkipp-Zughalters der Firma Gatan (Model 654) und Kupfer-Beryllium-Rahmen wird eine minimale Längenänderung der Probe von ca. $2 \mathrm{~nm}$ erreicht. In die CuBe-Rahmen wird mit dem Ionenstrahlmikroskop (engl. focused ion beam, FIB) ein ca. $5 \mu \mathrm{m}$ x $30 \mu \mathrm{m}$ großes Fenster geschnitten. Die Probe wird mit dem FIB soweit gedünnt, dass sie elektronentransparent ist, und anschließend über dem Fenster abgelegt und mit der Pt-Ionenstrahldeposition des FIB fixiert. Durch die PtDeposition wird die vorab gedünnte Probe wieder mit Platin bedeckt, welches nachträglich mit variierenden Ionenstrahlströmen in einer Argon-Ionenätze (Gatan DouMill) entfernt wird.

Die Verformung wird durch den Halter auf die CuBe-Rahmenstruktur aufgebracht. Da die $\mathrm{Ti} / \mathrm{ZrO}_{2}$-Probe mit diesem Rahmen fest verbunden ist, verformt sich die Probe gemeinsam mit dem Rahmen. Durch diese Co-Deformation ist es auch möglich, die geringen Schrittweiten der Verformung zu erreichen. Rahmen und Probe verformen sich nicht ab dem ersten Verfahrschritt des Halters. Erst ab einer gewissen Vorspannung von Halter und Rahmen sind Reaktionen der Probe auf die extern aufgebrachte Spannung zu beobachten. Der tatsächliche Zugversuch wird durch die kontinuierliche Elongation von Rahmen und Probe realisiert. Hier wird die kleinste Schrittweite verwendet und die Veränderung der Probe beobachtet und aufgezeichnet.

Abbildung B.1zeigt sowohl SEM- als auch TEM-Aufnahmen der Probe Ti100/ZrO 100 aufgeklebt auf die CuBe-Rahmenstruktur, jedoch noch ohne Verformung. Zu Beginn des Zugversuchs bewegen sich lediglich einige Biegekontraste im Siliciumsubstrat. Mit fortschreitender Verformung der Proben zeigt sich auch eine Reaktion innerhalb der Multilagen auf die aufgebrachte Spannung. Hier sind wie in den in-situ Bruchversuchen Kontrastwechsel in den kristallinen Ti-Schichten zu erkennen. Diese Kontrastwechsel 


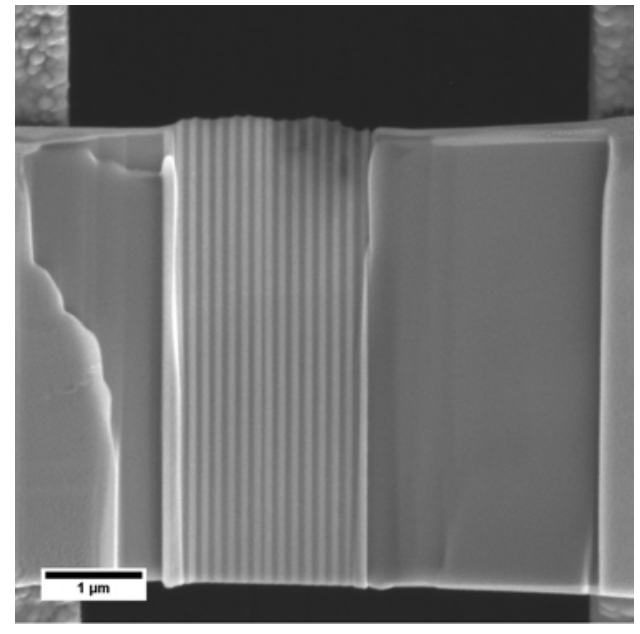

(a)

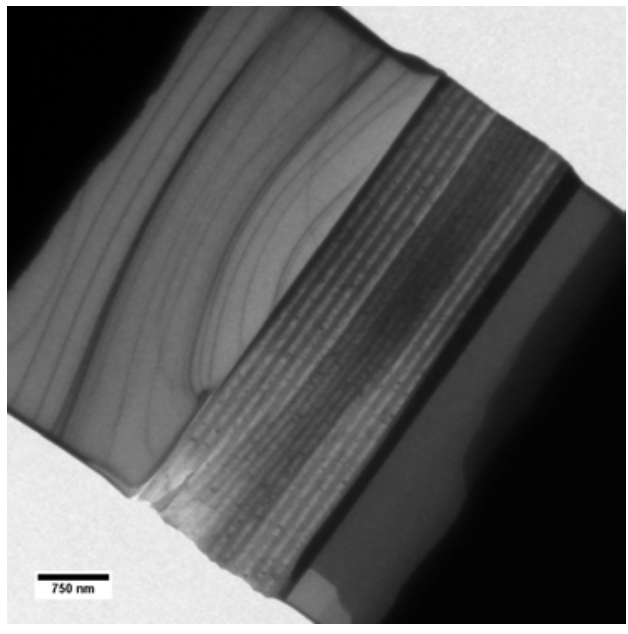

(b)

Abbildung B.1: Übersichtsaufnahmen des Zugversuchs: (a) SEM-Aufnahme nach der Präparation; (b) TEM-Aufnahme vor dem Zugversuch

sind homogen über die gesamte Lamelle verteilt. In Abbildung B.2 sind zwei Einzelbilder der Bildserie des gesamten Zugversuchs zu sehen. In Abbildung B.2a ist die Lamelle noch weitestgehend intakt. Es ist ein kleiner Riss innerhalb einer Ti-Schicht im linken unteren Bereich von Abbildung B.2a zu sehen. Weiterhin sind zwei der TiSchichten deutlich dünner (in Elektronenstrahlrichtung) als die restlichen, was sich durch den helleren Kontrast dieser Schichten zeigt. Ein Bild später (bei der gewählten Belichtungszeit entspricht das $100 \mathrm{~ms}$ ) ist die Lamelle in genau diesen beiden dünneren Ti-Schichten gerissen.

In einem zweiten Schritt wird die Spannung auf die Lamelle weiter erhöht und die schon vorhandenen Risse bewegen sich weiter. Auch hier läuft die weitere Rissbewegung innerhalb weniger Einzelbilder ab. Am Ende der Verformung ist eine Risskonfiguration, wie in Abbildung B.3a zu sehen, entstanden. Die Abbildungen B.3b bis B.3d zeigen höher vergrößerte Aufnahmen entlang der Rissoberfläche. Die weitere Analyse dieser Risse zeigt eindeutig, dass beide Risse im Ti initiiert (Abbildung B.3b) werden und sich auch in den Ti-Schichten ausgebreitet haben. An drei Stellen konnte vergleichbar zu den in-situ Bruchversuchen ein Abscheren der $\mathrm{ZrO}_{2}$-Schichten beobachtet werden, sodass der Riss an diesen Stellen in die benachbarte Ti-Schicht gewechselt ist (vgl. z.B. Abbildung B.3c). Eine quantitative Auswertung der Rissversuche ist aufgrund der Geschwindigkeit der Rissbildung und -bewegung nicht möglich. Rein qualitativ zeigt sich jedoch eine gute Übereinstimmung im Bruchverhalten des Zugversuchs im Vergleich zu den in-situ Bruchversuchen der Ti100/ $\mathrm{ZrO}_{2} 100$-Proben.

Im Rahmen dieser Arbeit werden keine weiteren Zugversuche an Proben mit dünneren Ti-Schichten durchgeführt, obwohl die Methodik problemlos auf dünnere Schichten übertragen werden könnte. Die wesentliche Limitierung der Zuggeometrie, wie sie hier 


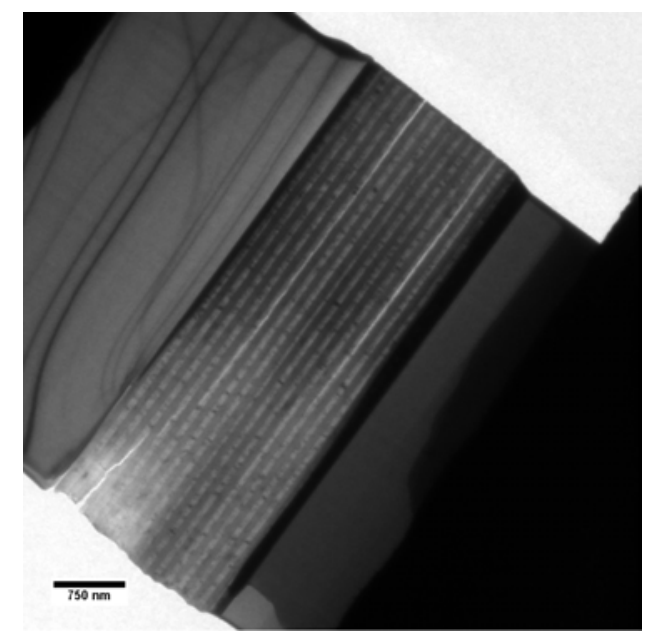

(a)

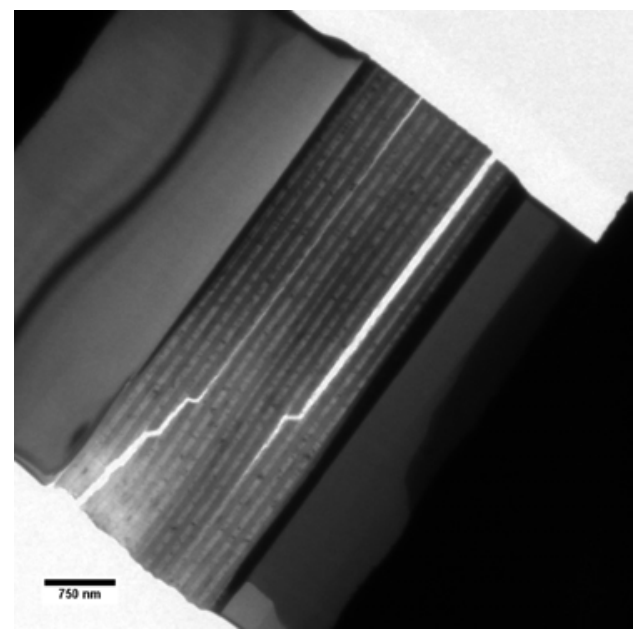

(b)

Abbildung B.2: TEM-Einzelbilder während des Zugversuchs; (a) Aufnahme vor der katastrophalen Rissbildung/-bewegung;(a) Aufnahme direkt nach der Rissbildung

realisiert wird, liegt darin, dass aufgrund der im System gespeicherten elastischen Energie mit einem katastrophalen Versagen der Lamellen zu rechnen ist. Die gespeicherte Energie ist so groß, dass, nachdem sich ein Riss gebildet hat, dieser instantan voran getrieben wird und daher keine Kontrolle über die Rissbewegung möglich ist. 


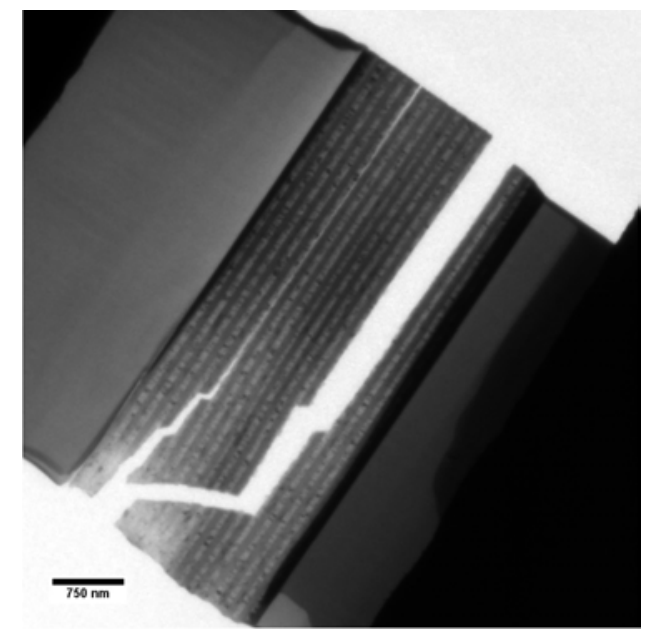

(a)

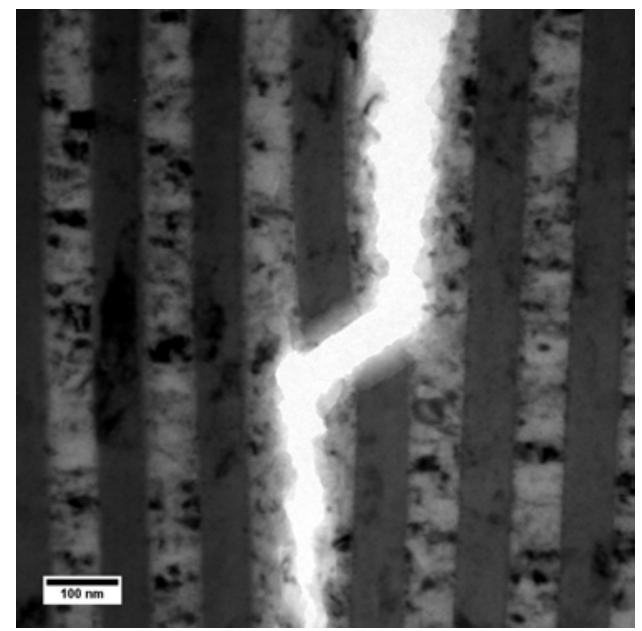

(c)

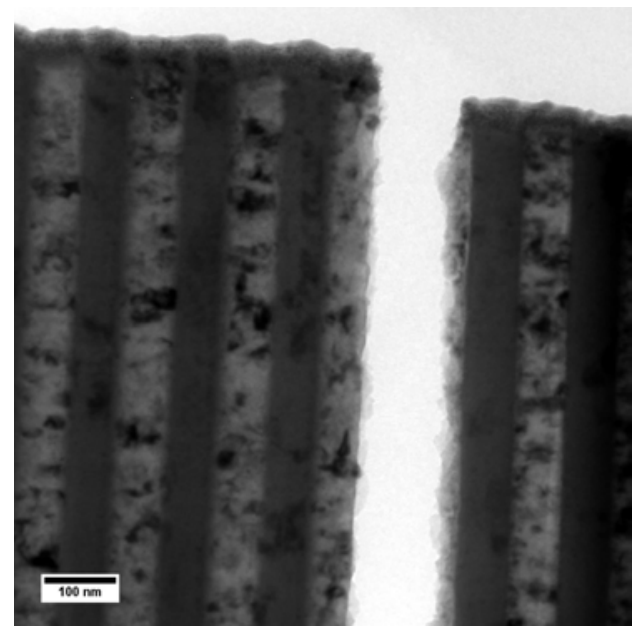

(b)

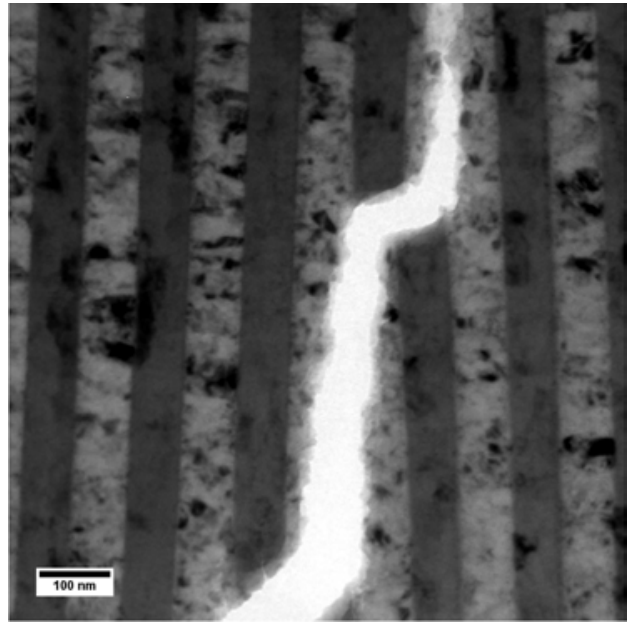

(d)

Abbildung B.3: Zugversuch der Ti100/ZrO 100 -Probe: (a) Risskonfiguration am Ende der Verformung; (b) (d) vergrößerte Aufnahmen entlang der entstanden Rissoberfläche 


\section{Kristallisation im FIB}

Es werden in verschiedensten Lamellen immer wieder Kontrastveränderungen im $\mathrm{ZrO}_{2}$ beobachtet. Ähnliche Kontrast wurden auch unabhängig von dieser Arbeit in TEM-Lamellen der hier verwendeten Proben festgestellt [Kno12]. Das $\mathrm{ZrO}_{2}$ wird bei Raumtemperatur deponiert und sollte daher amorph aufwachsen. Um den Ursprung dieser Kontrastveränderungen eindeutig zu klären, wird eine TEM-Lamelle der $\mathrm{ZrO}_{2^{-}}$ Einzelschicht entsprechend den typischen Präparationsschritten hergestellt und im TEM analysiert. Zusätzlich wird diese Einzelschicht absichtlich an einer bestimmten Position mit dem Ionenstrahl beleuchtet. Im TEM zeigen sich sowohl im ionenstrahlgeschädigten Bereich als auch an anderen Stellen Kontrastveränderungen, die auf eine Kristallisation des $\mathrm{ZrO}_{2}$ schließen lassen. Abbildung C.1a zeigt eine Hellfeldaufnahme der Lamelle. Es können eindeutig zwei Streifen mit Kontrastveränderungen am oberen Ende und in der Mitte der Lamelle beobachtet werden. Zusätzlich ist der absichtlich geschädigte Bereich markiert, in dem auch Kontrastveränderungen im Vergleich zum umgebenden Material vorhanden sind.

In Abbildung C.1b ist eine HRTEM-Aufnahme im illuminierten Bereich der Lamelle sowie die Fourier-Transformation der Aufnahme gezeigt. Im Realbild können eindeutig periodische Strukturen ausgemacht werden, die auf eine regelmäßige Anordnung der Atome zurückzuführen sind. Auch die Fourier-Transformation zeigt diskrete Reflexe, die durch Beugung der Elektronen an einem periodischen Gitter hervorgerufen werden, sodass auch hier Anzeichen von ionenstrahlinduzierten kristallinen Strukturen nachgewiesen werden können.

Der Ionenstrahl des FIB kann verschiedenste Schädigungen, wie Implantation von Ga-Ionen, Redeposition auf der Oberfläche als auch die Bildung einer amorphen Oberflächenschicht hervorrufen (z.B Kie07]). Im Fall des $\mathrm{ZrO}_{2}$ wird jedoch eine Kristallisation des amorphen Materials festgestellt. Eine ionenstrahlinduzierte Kristallisation amorpher $\mathrm{ZrO}_{2}$-Schichten konnte bereits beobachtet werden Hei11a, Lia09. Es ist dokumentiert, dass das amorphe Zirkonoxid metastabil aufwächst und bei 


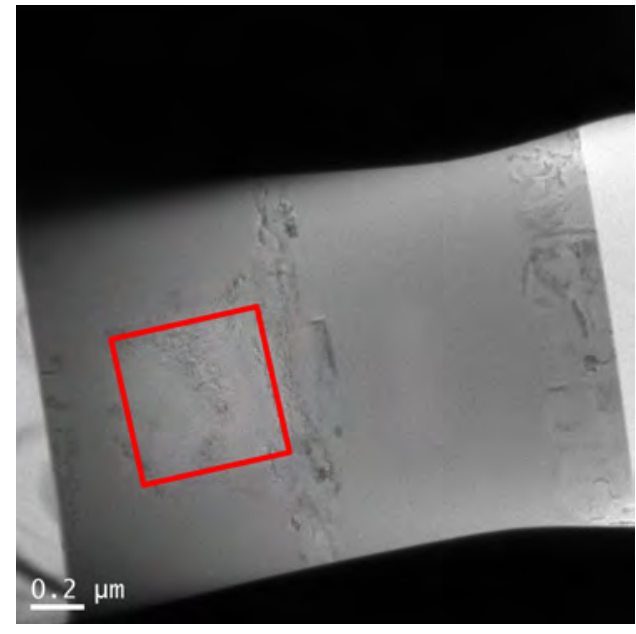

(a)

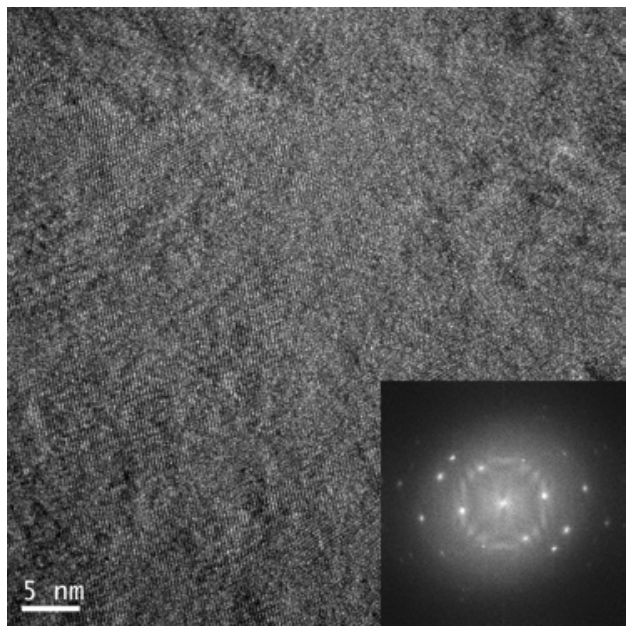

(b)

Abbildung C.1: $\mathrm{ZrO}_{2}$-Einzelschicht mit absichtlich FIB-geschädigtem Bereich: (a) Hellfeldaufnahme zeigt Kontrastveränderungen in der ursprünglich amorphen Struktur; (b) HRTEM-Aufnahme und FourierTransformation des mit dem lonenstrahl illuminierten Bereichs aus (a)

geringen Temperaturen von etwa $250^{\circ} \mathrm{C}$ kristallisieren kann. Allein schon der fokussierte Elektronenstrahl eines TEMs (Primärenergie der Elektronen von $300 \mathrm{keV}$ ) ermöglicht die Kristallisation der amorphen $\mathrm{ZrO}_{2}$-Schichten Hei11a, Kno12. Bei den Untersuchungen (auch STEM-Analysen) in dieser Arbeit konnte kein Einfluss eines fokussierten Elektronenstrahls auf die Kristallisation der $\mathrm{ZrO}_{2}$-Schichten beobachtet werden.

Durch die mechanische Beanspruchung der jeweils oberen $\mathrm{ZrO}_{2}$-Schicht in den $\mathrm{Ti} / \mathrm{ZrO}_{2}$-Multilagenproben in $\mathrm{Kno12}$ wird eine Kristallisation dieser $\mathrm{ZrO}_{2}$-Schicht beobachtet, die im Kontakt mit der Indenterspitze ist. Diese lokalisierte Kristallisation wird in Verbindung mit einem möglichen Energieeintrag durch die aufgebrachten Spannungen oder der herrschenden Reibung zwischen der $\mathrm{ZrO}_{2}$-Schicht und der Indenterspitze gebracht.

Weiterhin wird in der Literatur von weiteren Experimenten berichtet, in denen amorph-kristalline $\mathrm{ZrO}_{2}$-Multilagen mit $\mathrm{Kr}$-Ionen beschossen (Energie $1 \mathrm{MeV}$ ) wurden und es konnte eine Phasentransformation der amorphen Schichten hin zu tetragonalkristallinen Schichten nachgewiesen werden. Diese Phasentransformation tritt bei Raumtemperatur auf. Auch in $\mathrm{ZrHfOCH}-F i l m e n$ auf einkristallinen $\mathrm{ZrO}_{2}$-Substraten wird Kristallisation beobachtet [Yu96. Bei einer Temperatur von $300{ }^{\circ} \mathrm{C}$ und unter dem Beschuss verschiedener Ionen und Energien (Ar, Ne und O) werden die ursprünglich amorphen Schichten kristallisiert. An der Grenzfläche zum Substrat kristallisiert die Schicht epitaktisch und geht mit größerem Abstand zum Substrat in eine polykristalline Struktur über. 


\section{Literaturverzeichnis}

[And69] Anderson, WE, Some Designer-Oriented Views on Brittle Fracture, Pacific Northwest National Laboratory (PNNL), Richland, WA, 1969.

[And95] Anderson, T, Fracture Mechanics - Fundamentals and Applications, CRC Press LLC, 1995.

[And09] Andrievski, RA und Glezer, AM, Strength of nanostructures, Physics-Uspekhi, 52 (4), 315, 2009.

[Arg79] Argon, AS, Plastic deformation in metallic glasses, Acta Metallurgica, 27 (1), 47-58, 1979.

[Arm11] Arman, B; Brandl, C; Luo, SN; Germann, TC; Misra, A und Çagin, T, Plasticity in $\mathrm{Cu}(111) / \mathrm{Cu}_{46} \mathrm{Zr}_{54}$ glass nanolaminates under uniaxial compression, Journal of Applied Physics, 110 (4), 043539, 2011.

[Arz03] Arzt, E; Gorb, S und Spolenak, R, From micro to nano contacts in biological attachment devices., Proceedings of the National Academy of Sciences of the United States of America, 100 (19), 10603-6, 2003.

[AST13] ASTM E1820-13e1, Standard Test Method for Measurement of Fracture Toughness, ASTM International, West Conshohocken, PA, 2013.

[Ast14] Ast, J; Przybilla, T; Maier, V; Durst, K und Göken, M, Microcantilever bending experiments in NiAl - Evaluation, size effects, and crack tip plasticity, Journal of Materials Research, 29, 2129-2140, 2014.

[Bag94] Bagchi, A; Lucas, G; Suo, Z und Evans, A, A new procedure for measuring the decohesion energy for thin ductile films on substrates, Journal of Materials Research, 9, 1734-1741, 1994.

[Bag96] Bagchi, A und Evans, A, Measurements of the debond energy for thin metallization lines on dielectrics, Thin Solid Films, 286 (1-2), 203-212, 1996. 
[Bai99] Baither, D; Ernst, F; Wagner, T; Rühle, M; Bartsch, M und Messerschmidt, U, Micromechanisms of fracture in NiAl studied by in situ straining experiments in an HVEM, Intermetallics, 7 (3-4), 479-489, 1999.

[Bar09] Barnoush, A, Hydrogen embrittlement - Revisited by in situ electrochemical nanoindentation, Shaker Verlag GmbH, Deutschland, 2009.

[Bea11] Beaber, AR; Nowak, JD; Ugurlu, O; Mook, WM; Girshick, SL; Ballarini, $\mathrm{R}$ und Gerberich, WW, Smaller is tougher, Philosophical Magazine, 91 (7), 1179-1189, 2011.

[Bir94] Birnbaum, H und Sofronis, P, Hydrogen-enhanced localized plasticity - a mechanism for hydrogen-related fracture, Materials Science and Engineering: A, 176 (1), 191-202, 1994.

[Bro73] Broek, D und Vlieger, H, The thickness effect in plane stress fracture toughness, Technischer Bericht, National Aerospace Laboratory NLR, 1973.

[Bud04] Budrovic, Z; Van Swygenhoven, H; Derlet, P; Petegem, S und Schmitt, B, Plastic deformation with reversible peak broadening in nanocrystalline $\mathrm{Ni}$, Science, 309, 273-276, 2004.

[Cah83] Cahn, R und Haasen, P, Physical metallurgy, Elsevier Science, Amsterdam, 1983.

[Cao91] Cao, H und Evans, A, On crack extension in ductile/brittle laminates, Acta Metallurgica et Materialia, 39 (12), 2997-3005, 1991.

[Car07] Carlton, C und Ferreira, P, What is behind the inverse Hall-Petch effect in nanocrystalline materials?, Acta Materialia, 55 (11), 3749-3756, 2007.

[Cha98] Chase, MW, NIST-JANAF thermochemical tables, American Chemical Society ; American Institute of Physics for the National Institute of Standards and Technology, [Washington, D.C.]; Woodbury, N.Y., 1998.

[Che05a] Cheng, S; Ma, E; Wang, Y; Kecskes, L; Youssef, K; Koch, C; Trociewitz, U und Han, K, Tensile properties of in situ consolidated nanocrystalline $\mathrm{Cu}$, Acta Materialia, 53 (5), 1521-1533, 2005.

[Che05b] Chevalier, J und Fantozzi, G, Slow Crack Propagation in Ceramics at the Nano- and Micro-Scale: Effect of the Microstructure, in Bradt, R; Munz, D; Sakai, M und White, K, Hg., Fracture Mechanics of Ceramics, Band 14 von Fracture Mechanics of Ceramics, 173-190, Springer US, 2005.

[Che09] Chevalier, J; Gremillard, L; Virkar, AV und Clarke, DR, The TetragonalMonoclinic Transformation in Zirconia: Lessons Learned and Future Trends, Journal of the American Ceramic Society, 92 (9), 1901-1920, 2009.

[Cho89] Chokshi, A; Rosen, A; Karch, J und Gleiter, H, On the validity of the HallPetch relationship in nanocrystalline materials, Scripta Metallurgica, 23 (10), 1679-1683, 1989. 
[Cho99] Choi, SR; Hutchinson, JW und Evans, A, Delamination of multilayer thermal barrier coatings, Mechanics of Materials, 31 (7), 431-447, 1999.

[Col66] Coleman, C und Hardie, D, The hydrogen embrittlement of $\alpha$-zirconium - A review, Journal of the Less Common Metals, 11 (3), 168-185, 1966.

[Dal89] Dalgleish, B; Trumble, K und Evans, A, The strength and fracture of alumina bonded with aluminum alloys, Acta Metallurgica, 37 (7), 1923-1931, 1989.

[Dau98] Dauskardt, R; Lane, M; Ma, Q und Krishna, N, Adhesion and debonding of multi-layer thin film structures, Engineering Fracture Mechanics, 61 (1), 141-162, 1998.

[Deh02] Dehm, G; B. J. Inkson, TB, T. Wagner und Arzt, E, Plasticity and Interfacial Dislocation Mechanisms in Epitaxial and Polycrystalline Al Films Constrained by Substrates, Journal Materials Science and Technology, 18, 113-117, 2002.

[Deh06] Dehm, G; Motz, C; Scheu, C; Clemens, H; Mayrhofer, P und Mitterer, C, Mechanical Size-Effects in Miniaturized and Bulk Materials, Advanced Engineering Materials, 8 (11), 1033-1045, 2006.

[Den05] Deng, X; Chawla, N; Chawla, K; Koopman, M und Chu, J, Mechanical Behavior of Multilayered Nanoscale Metal-Ceramic Composites, Advanced Engineering Materials, 7 (12), 1099-1108, 2005.

[DM05] Di Maio, D und Roberts, S, Measuring fracture toughness of coatings using focused-ion-beam-machined microbeams, Journal of Materials Research, 20, 299-302, 2005.

[Dol15] Dolan, MD; Kochanek, MA; Munnings, CN; McLennan, KG und Viano, DM, Hydride phase equilibria in V-Ti-Ni alloy membranes, Journal of Alloys and Compounds, 622, 276-281, 2015.

[Don00] Donachie, MJ, Titanium: A Technical Guide, ASM International, 2000.

[Dun69] Dundurs, J, Edge-Bonded Dissimilar Orthogonal Elastic Wedges Under Normal and Shear Loading, Journal of Applied Mechanics, 36 (3), 650-652, 1969.

[Eva99] Evans, A; Hutchinson, J und Wei, Y, Interface adhesion: Effects of plasticity and segregation, Acta Materialia, 47 (15-16), 4093-4113, 1999.

[FEI13] FEI Electron Optics, Certificate of instrument quality Titan 80-300 serial number D3302, FEI Electron Optics, Eindhoven, The Netherlands, 2013.

[Fle91] Fleck, NA; Hutchinson, JW und Zhigang, S, Crack path selection in a brittle adhesive layer, International Journal of Solids and Structures, 27 (13), 1683$1703,1991$.

[Gao03] Gao, H; Ji, B; Jäger, IL; Arzt, E und Fratzl, P, Materials become insensitive to flaws at nanoscale: Lessons from nature, Proceedings of the National Academy of Sciences, 100 (10), 5597-5600, 2003. 
[Ger83] Gerkema, J und Miedema, A, Adhesion between solid metals: obsevations of intrefacial segregation effects in metal film lubrication experiments, Surface Science, 124, 351-371, 1983.

[Ger09] Gerberich, W; Michler, J; Mook, W; Ghisleni, R; Östlund, F; Stauffer, D und Ballarini, R, Scale effects for strength, ductility, and toughness in brittle materials, Journal of Materials Research, 24, 898-906, 2009.

[Got07] Gottstein, G, Physikalische Grundlagen der Materialkunde, Springer, 3rd edition Auflage, 2007.

[Gra92] Graef, MD; Dalgleish, B; Turner, M und Evans, A, Interfaces between alumina and platinum: Structure, bonding and fracture resistance, Acta Metallurgica et Materialia, 40, S333-S344, 1992.

[Gra95] Grabke, HJ; Kurbatov, G und Schmutzler, HJ, Segregation beneath oxide scales, Oxidation of Metals, 43 (1-2), 97-114, 1995.

[Gra13] Granta Design Limited, CES Selector software, Cambridge, UK, 2013.

[Gre11] Greer, JR und Hosson, JTD, Plasticity in small-sized metallic systems: Intrinsic versus extrinsic size effect, Progress in Materials Science, 56 (6), 654-724, 2011 .

[Gri20] Griffith, A, The Phenomena of Rupture and Flow in Solids, Philosophical Transactions Series A, 221, 163-198, 1920.

[Gui96] Guinea, G; Elices, M und Planas, J, Stress intensity factors for wedge-splitting geometry, International Journal of Fracture, 81 (2), 113-124, 1996.

[Guo04] Guo, X, Property Degradation of Tetragonal Zirconia Induced by LowTemperature Defect Reaction with Water Molecules, Chemistry of Materials, 16 (21), 3988-3994, 2004.

[Hac91] Hackney, S und Milligan, W, A new in situ technique for studying deformation and fracture in thin film ductile/brittle laminates, Ultramicroscopy, 37, 7989, 1991.

[Haf07] Hafner, B, Scanning Electron Microscopy Primer, Characterization Facility, University of Minnesota-Twin Cities, 2007.

[Haf11] Hafner, B, Introductory Transmission Electron Microscopy Primer, Characterization Facility, University of Minnesota-Twin Cities, 2011.

[Han10] Hansen, TW; Wagner, JB und Dunin-Borkowski, RE, Aberration corrected and monochromated environmental transmission electron microscopy: challenges and prospects for materials science, Materials Science and Technology, 26 (11), 1338-1344, 2010.

[Haq04] Haque, MA und Saif, MTA, Deformation mechanisms in free-standing nanoscale thin films: A quantitative in situ transmission electron microscope study, Proceedings of the National Academy of Sciences of the United States of America, 101 (17), 6335-6340, 2004. 
[Hei10] Heiroth, S; Lippert, T; Wokaun, A; Döbeli, M; Rupp, J; Scherrer, B und Gauckler, L, Yttria-stabilized zirconia thin films by pulsed laser deposition: Microstructural and compositional control, Journal of the European Ceramic Society, 30 (2), 489-495, 2010.

[Hei11a] Heiroth, S; Frison, R; Rupp, JL; Lippert, T; Meier, EJB; Gubler, EM; Döbeli, M; Conder, K; Wokaun, A und Gauckler, LJ, Crystallization and grain growth characteristics of yttria-stabilized zirconia thin films grown by pulsed laser deposition, Solid State Ionics, 191 (1), 12-23, 2011.

[Hei11b] Heiroth, S; Ghisleni, R; Lippert, T; Michler, J und Wokaun, A, Optical and mechanical properties of amorphous and crystalline yttria-stabilized zirconia thin films prepared by pulsed laser deposition, Acta Materialia, 59 (6), 23302340, 2011.

[Hir06] Hirakata, H; Kitazawa, M und Kitamura, T, Fatigue crack growth along interface between metal and ceramics submicron-thick films in inert environment, Acta Materialia, 54 (1), 89-97, 2006.

[Hou05] Hou, P und Priimak, K, Interfacial Segregation, Pore Formation, and Scale Adhesion on NiAl Alloys, Oxidation of Metals, 63 (1-2), 113-130, 2005.

[How12] Howie, PR; Korte, S und Clegg, WJ, Fracture modes in micropillar compression of brittle crystals, Journal of Materials Research, 27, 141-151, 2012.

[Hsi94] Hsia, K; Suo, Z und Yang, W, Cleavage due to dislocation confinement in layered materials, Journal of the Mechanics and Physics of Solids, 42 (6), 877-896, 1994.

[Hua95] Huang, Y und Zhang, H, The role of metal plasticity and interfacial strength in the cracking of metal/ceramic laminates, Acta Metallurgica et Materialia, 43 (4), 1523-1530, 1995.

[Hut92] Hutchinson, JW und Suo, Z, Mixed Mode Cracking in Layered Materials, Advances in Applied Mechanics, 29, 1992.

[Hwu99] Hwu, K und Derby, B, Fracture of metal/ceramic laminates-II. Crack growth resistance and toughness, Acta Materialia, 47 (2), 545-563, 1999.

[Ing13] Inglis, C, Stresses in a plate due to the presence of cracks and sharp corners, Trans. Inst. Naval Architects, 219-230, 1913.

[Ing86] Ingel, RP und Lewis, D, Lattice Parameters and Density for $\mathrm{Y}_{2} \mathrm{O}_{3}$-Stabilized $\mathrm{ZrO}_{2}$, Journal of the American Ceramic Society, 69 (4), 325-332, 1986.

[Ino08] Inoue, J; Nambu, S; Ishimoto, Y und Koseki, T, Fracture elongation of brittle/ductile multilayered steel composites with a strong interface, Scripta Materialia, 59 (10), 1055-1058, 2008.

[Iqb12] Iqbal, F; Ast, J; Göken, M und Durst, K, In situ micro-cantilever tests to study fracture properties of NiAl single crystals, Acta Materialia, 60 (3), $1193-1200,2012$. 
[Irw48] Irwin, G, Fracture Dynamics, Fracturing of Metals, A.S.M., Cleveland, 147166, 1948.

[Jay14] Jaya, B und Jayaram, V, Crack stability in edge-notched clamped beam specimens: modeling and experiments, International Journal of Fracture, 188 (2), 213-228, 2014.

[Jay15] Jaya, BN; Kirchlechner, C und Dehm, G, Can microscale fracture tests provide reliable fracture toughness values? A case study in silicon, Journal of Materials Research, 30, 686-698, 2015.

[Jha98] Jha, M und Charalambides, P, A finite element analysis of fracture initiation in ductile/brittle periodically layered composites, International Journal of Fracture, 90 (4), 299-323, 1998.

[Ji10] Ji, B und Gao, H, Mechanical Principles of Biological Nanocomposites, Annual Review of Materials Research, 40 (1), 77-100, 2010.

[Jia06] Jiang, HY; Kelch, S und Lendlein, A, Polymers Move in Response to Light, Advanced Materials, 18 (11), 1471-1475, 2006.

[Kam00] Kamat, S; Su, X; Ballarini, R und Heuer, AH, Structural basis for the fracture toughness of the shell of the conch Strombus gigas, Nature, 405 (6790), 1036$1040,2000$.

[Kan05] Kang, YL; Zhang, ZF; Wang, HW und Qin, QH, Experimental investigations of the effect of thickness on fracture toughness of metallic foils, Materials Science and Engineering: A, 394 (1-2), 312-319, 2005.

[Kap11] Kapelle, B, In situ Zugversuch an einkristallinen Gold-Nanodrähten im REM, Diplomarbeit, Georg-August-Universität Göttingen, 2011.

[Kie07] Kiener, D; Motz, C; Rester, M; Jenko, M und Dehm, G, FIB damage of $\mathrm{Cu}$ and possible consequences for miniaturized mechanical tests, Materials Science and Engineering: A, 459 (1-2), 262-272, 2007.

[Kim10] Kim, S; Wu, J; Carlson, A; Jin, SH; Kovalsky, A; Glass, P; Liu, Z; Ahmed, N; Elgan, SL; Chen, W; Ferreira, PM; Sitti, M; Huang, Y und Rogers, JA, Microstructured elastomeric surfaces with reversible adhesion and examples of their use in deterministic assembly by transfer printing., Proceedings of the National Academy of Sciences of the United States of America, 107 (40), 17095-100, 2010.

[Kno12] Knorr, I, Mechanische Eigenschaften kristallin-amorpher Schichtpakete, Doktorarbeit, Georg-August-Universität Göttingen, 2012.

[Kno13] Knorr, I; Cordero, N; Lilleodden, E und Volkert, C, Mechanical behavior of nanoscale Cu/PdSi multilayers, Acta Materialia, 61 (13), 4984-4995, 2013.

[Koc07] Koch, C, Structural nanocrystalline materials: an overview, Journal of Materials Science, 42 (5), 1403-1414, 2007. 
[Kra10] Kraft, O; Gruber, PA; Mönig, R und Weygand, D, Plasticity in Confined Dimensions, Annual Review of Materials Research, 40, 293-317, 2010.

[Kru04] Kruzic, J; McNaney, J; Cannon, R und Ritchie, R, Effects of plastic constraint on the cyclic and static fatigue behavior of metal/ceramic layered structures, Mechanics of Materials, 36 (1-2), 57-72, 2004.

[KS96] Kuhn-Spearing, L; Kessler, H; Chateau, E; Ballarini, R; Heuer, A und Spearing, S, Fracture mechanisms of the Strombus gigas conch shell: implications for the design of brittle laminates, Journal of Materials Science, 31 (24), 6583-6594, 1996.

[Kum03] Kumar, K; Swygenhoven, HV und Suresh, S, Mechanical behavior of nanocrystalline metals and alloys, Acta Materialia, 51 (19), 5743-5774, 2003.

[Kum09] Kumar, S; Haque, MA und Gao, H, Notch insensitive fracture in nanoscale thin films, Applied Physics Letters, 94 (25), 253104, 2009.

[Kum10] Kumar, S; Zhuo, D; Wolfe, D; Eades, J und Haque, M, Length-scale effects on fracture of multilayers, Scripta Materialia, 63 (2), 196-199, 2010.

[Kum11] Kumar, S; Li, X; Haque, A und Gao, H, Is Stress Concentration Relevant for Nanocrystalline Metals?, Nano Letters, 11 (6), 2510-2516, 2011.

[Kun04] Kuntz, JD; Zhan, GD und Mukherjee, AK, Nanocrystalline-Matrix Ceramic Composites for Improved Fracture Toughness, MRS Bulletin, 29, 22-27, 2004.

[Kür12] Kürschner, J, Electrochemical Hydrogenography of metal thin films, Masterarbeit, 2012.

[Lan00] Lane, M; Dauskardt, RH; Krishna, N und Hashim, I, Adhesion and reliability of copper interconnects with Ta and TaN barrier layers, Journal of Materials Research, 15, 203-211, 2000.

[Lan03] Lane, M, Interface Fracture, Annual Review of Materials Research, 33 (1), 29-54, 2003.

[Lea82] Leapman, RD; Grunes, LA und Fejes, PL, Study of the $L_{23}$ edges in the $3 d$ transition metals and their oxides by electron-energy-loss spectroscopy with comparisons to theory, Phys. Rev. B, 26, 614-635, 1982.

[Lee07] Lee, H; Lee, BP und Messersmith, PB, A reversible wet/dry adhesive inspired by mussels and geckos, Nature, 448 (7151), 338-341, 2007.

[Li04] Li, H und Ebrahimi, F, Transition of deformation and fracture behaviors in nanostructured face-centered-cubic metals, Applied Physics Letters, 84 (21), 4307-4309, 2004.

[Li05] Li, H und Ebrahimi, F, Ductile-to-Brittle Transition in Nanocrystalline Metals, Advanced Materials, 17 (16), 1969-1972, 2005. 
[Lia09] Lian, J; Zhang, J; Namavar, F; Zhang, Y; Lu, F; Haider, H; Garvin, K; Weber, WJ und Ewing, RC, Ion beam-induced amorphous-to-tetragonal phase transformation and grain growth of nanocrystalline zirconia, Nanotechnology, 20 (24), 245303, 2009.

[Lie07] Liese, T, Wachstum und Grenzflächenrauigkeit laserdeponierter MgO/Ti- und $\mathrm{ZrO}_{2} /$ Ti-Multischichtsysteme, Diplomarbeit, Georg-August-Universität Göttingen, 2007.

[Lie11] Liese, T; Radisch, V; Knorr, I; Reese, M; Großmann, P; Mann, K und Krebs, HU, Development of laser deposited multilayer zone plate structures for soft X-ray radiation, Applied Surface Science, 257, 5138-5141, 2011.

[Lie12] Liese, T, Multilagenbasierte Transmissionsoptiken für die Röntgenmikroskopie, Doktorarbeit, Georg-August-Universität Göttingen, 2012.

[Lip98] Lipkin, D; Clarke, D und Evans, A, Effect of interfacial carbon on adhesion and toughness of gold-sapphire interfaces, Acta Materialia, 46 (13), 48354850, 1998.

[Liu96] Liu, H und Hsu, SM, Fracture Behavior of Multilayer Silicon Nitride/Boron Nitride Ceramics, Journal of the American Ceramic Society, 79 (9), 24522457, 1996.

[Liu12] Liu, M; Du, X; Lin, I; Pei, H und Huang, J, Superplastic like deformation in metallic amorphous/crystalline nanolayered micropillars, Intermetallics, 30 (0), 30-34, 2012.

[Liu13] Liu, S; Wheeler, JM; Howie, PR; Zeng, XT; Michler, J und Clegg, WJ, Measuring the fracture resistance of hard coatings, Applied Physics Letters, 102 (17), 171907, 2013.

[Low04] Lowe, DG, Distinctive Image Features from Scale-Invariant Keypoints, Int. J. Comput. Vision, 60 (2), 91-110, 2004.

[Lu00] Lu, G; Bernasek, SL und Schwartz, J, Oxidation of a polycrystalline titanium surface by oxygen and water, Surface Science, 458 (1-3), 80-90, 2000.

[Lu07] Lu, N; Wang, X; Suo, Z und Vlassak, J, Metal films on polymer substrates stretched beyond 50\%, Applied Physics Letters, 91 (22), 221909, 2007.

[Lüt00] Lütjering, G; Williams, J und Gysler, A, Microstructure and mechanical properties of titanium alloys, World Scientific, 2000.

[Mal88] Malis, T; Cheng, SC und Egerton, RF, EELS log-ratio technique for specimenthickness measurement in the TEM, Journal of Electron Microscopy Technique, 8 (2), 193-200, 1988.

[Mat12] Matsuda, Y; King, S; Bielefeld, J; Xu, J und Dauskardt, R, Fracture properties of hydrogenated amorphous silicon carbide thin films, Acta Materialia, 60 (2), 682-691, 2012. 
[McN96] McNaney, J; Cannon, $\mathrm{R}$ und Ritchie, R, Fracture and fatigue-crack growth along aluminum-alumina interfaces, Acta Materialia, 44 (12), 4713-4728, 1996.

[Mec15] Meckenhäuser, C, In-situ TEM fracture tests of nanoscale multilayers, Masterarbeit, 2015.

[Mei12] Meirom, RA; Clark, TE und Muhlstein, CL, The role of specimen thickness in the fracture toughness and fatigue crack growth resistance of nanocrystalline platinum films, Acta Materialia, 60 (3), 1408-1417, 2012.

[Mer11] Merle, B und Göken, M, Fracture toughness of silicon nitride thin films of different thicknesses as measured by bulge tests, Acta Materialia, 59 (4), 1772-1779, 2011.

[Mer14] Merle, B und Göken, M, Bulge fatigue testing of freestanding and supported gold films, Journal of Materials Research, 29, 267-276, 2014.

[Mey06] Meyers, M; Mishra, A und Benson, D, Mechanical properties of nanocrystalline materials, Progress in Materials Science, 51 (4), 427-556, 2006.

[Mis03] Misra, A; Kung, H; Hammon, D; Hoagland, RG und Nastasi, M, Damage Mechanisms in Nanolayered Metallic Composites, International Journal of Damage Mechanics, 12 (4), 365-376, 2003.

[Mis07] Misra, A und Hoagland, R, Plastic flow stability of metallic nanolaminate composites, Journal of Materials Science, 42 (5), 1765-1771, 2007.

[Moo07] Mook, WM; Nowak, JD; Perrey, CR; Carter, CB; Mukherjee, R; Girshick, SL; McMurry, PH und Gerberich, WW, Compressive stress effects on nanoparticle modulus and fracture, Physical Review B, 75 (21), 214112, 2007.

[Mor97] Morris, M und Leboeuf, M, Grain-size refinement of gamma-Ti-Al alloys: effect on mechanical properties, Materials Science and Engineering: A, 224 (12), 1-11, 1997.

[Mos06a] Moser, B; Hanlon, T; Kumar, K und Suresh, S, Cyclic strain hardening of nanocrystalline nickel, Scripta Materialia, 54 (6), 1151-1155, 2006.

[Mos06b] Moser, B; Löffler, JF und Michler, J, Discrete deformation in amorphous metals: an in situ SEM indentation study, Philosophical Magazine, 86 (3335), 5715-5728, 2006.

[Muk07] Mukhopadhyay, A und Basu, B, Consolidation-microstructure-property relationships in bulk nanoceramics and ceramic nanocomposites: a review, International Materials Reviews, 52 (5), 257-288, 2007.

[Mus82] Mussler, B; Swain, M und Claussen, N, Dependence of Fracture Toughness of Alumina on Grain Size and Test Technique, Journal of the American Ceramic Society, 65 (11), 566-572, 1982. 
[Nag99] Nagel, R; Hahn, H und Balogh, A, Diffusion processes in metal/ceramic interfaces under heavy ion irradiation, Nuclear Instruments and Methods in Physics Research Section B: Beam Interactions with Materials and Atoms, 148, 930-935, 1999.

[Nix98] Nix, WD, Yielding and strain hardening of thin metal films on substrates, Scripta Materialia, 39, 545-554, 1998.

[Nor08] Northen, MT; Greiner, C; Arzt, E und Turner, KL, A Gecko-Inspired Reversible Adhesive, Advanced Materials, 20 (20), 3905-3909, 2008.

[Now07] Nowak, JD; Mook, WM; Minor, AM; Gerberich, WW und Carter, CB, Fracturing a nanoparticle, Philosophical Magazine, 87 (1), 29-37, 2007.

[Oga04] Ogata, S; Li, J; Hirosaki, N; Shibutani, Y und Yip, S, Ideal shear strain of metals and ceramics, Physical Review B, 70 (10), 104104, 2004.

[Öst09] Östlund, F; Rzepiejewska-Malyska, K; Leifer, K; Hale, LM; Tang, Y; Ballarini, R; Gerberich, WW und Michler, J, Brittle-to-Ductile Transition in Uniaxial Compression of Silicon Pillars at Room Temperature, Advanced Functional Materials, 19 (15), 2439-2444, 2009.

[Öst11] Östlund, F; Howie, PR; Ghisleni, R; Korte, S; Leifer, K; Clegg, WJ und Michler, J, Ductile-brittle transition in micropillar compression of GaAs at room temperature, Philosophical Magazine, 91 (7-9), 1190-1199, 2011.

[Ovi07] Ovid'ko, I, Review on the fracture processes in nanocrystalline materials, Journal of Materials Science, 42 (5), 1694-1708, 2007.

[Ovi11] Ovid'ko, I und Sheinerman, A, Micromechanisms for improved fracture toughness in nanoceramics, Reviews on advanced Materials Science, 29 (1), 105$125,2011$.

[Ovi15] Ovid'ko, IA, Micromechanics of fracturing in nanoceramics, Philosophical Transactions of the Royal Society of London A: Mathematical, Physical and Engineering Sciences, 373 (2038), 2015.

[Par00] Park, SY; Saruhan, B und Schneider, H, Mullite/zirconia laminate composites for high temperature application, Journal of the European Ceramic Society, 20 (14-15), 2463-2468, 2000.

[Pio14] Piotrowski, T, Recycling faserverstärkter Kunststoffe: Existierende Methoden und Konzepte für die Zukunft, Masterarbeit, 2014.

[Pun03] Pundt, A und Pekarski, P, Buckling of thin niobium-films on polycarbonate substrates upon hydrogen loading, Scripta Materialia, 48 (4), 419-423, 2003.

[Pun04] Pundt, A; Nikitin, E; Pekarski, P und Kirchheim, R, Adhesion energy between metal films and polymers obtained by studying buckling induced by hydrogen, Acta Materialia, 52 (6), 1579-1587, 2004. 
[Raa12] Raabe, S; Mierwaldt, D; Ciston, J; Uijttewaal, M; Stein, H; Hoffmann, J; Zhu, Y; Blöchl, P und Jooss, C, In Situ Electrochemical Electron Microscopy Study of Oxygen Evolution Activity of Doped Manganite Perovskites, Advanced Functional Materials, 22 (16), 3378-3388, 2012.

[Red07] Reddy, S; Arzt, E und del Campo, A, Bioinspired Surfaces with Switchable Adhesion, Advanced Materials, 19 (22), 3833-3837, 2007.

[Rei90] Reimanis, I; Dalgleish, B und Brahy, M, Effects of plasticity on the crack propagation resistance of a metal/ceramic interface, Acta Metallurgica et Materialia, 38 (12), 2645-2652, 1990.

[Rei91] Reimanis, I; Dalgleish, B und Evans, A, The fracture resistance of a model metal/ceramic interface, Acta Metallurgica et Materialia, 39 (12), 3133-3141, 1991.

[Ric68] Rice, J, A Path Independent Intergral and the Approximate Analysis of Strain Concentration by Notches and Cracks, Journal of Applied Mechanics, 35, 379-386, 1968.

[Rit93] Ritchie, R; Cannon, R; Dalgleish, B; Dauskardt, R und McNaney, J, Mechanics and mechanisms of crack growth at or near ceramic-metal interfaces: interface engineering strategies for promoting toughness, Materials Science and Engineering: A, 166 (1-2), 221-235, 1993.

[Röd08] Röder, J und Krebs, HU, Frequency dependent smoothing of rough surfaces by laser deposition of $\mathrm{ZrO}_{2}$, Applied Physics A, 90 (4), 609-613, 2008.

[Sch92] Schmauder, S und Meyer, M, Correlation between Dundurs' parameters and elastic constants, Zeitschrift für Metallkunde, 83 (7), 524-527, 1992.

[Sch12a] Schaufler, J; Schmid, C; Durst, K und Göken, M, Determination of the interfacial strength and fracture toughness of a-C:H coatings by in-situ microcantilever bending, Thin Solid Films, 522 (0), 480-484, 2012.

[Sch12b] Schneider, CA; Rasband, WS und Eliceiri, KW, NIH Image to ImageJ: 25 years of image analysis, Nature Methods, 9 (7), 671-675, 2012.

[Sea79] Seah, MP, Quantitative prediction of surface segregation, Journal of Catalysis, 57 (3), 450-457, 1979.

[Set04] Setoyama, D; Matsunaga, J; Muta, H; Uno, M und Yamanaka, S, Mechanical properties of titanium hydride, Journal of Alloys and Compounds, 381 (1-2), 215-220, 2004.

[Sha04] Shan, Z; Stach, EA; Wiezorek, JMK; Knapp, JA; Follstaedt, DM und Mao, SX, Grain Boundary-Mediated Plasticity in Nanocrystalline Nickel, Science, 305 (5684), 654-657, 2004.

[Shi06] Shimizu, F; Ogata, S und Li, J, Yield point of metallic glass, Acta Materialia, 54 (16), 4293-4298, 2006. 
[Sin11] Singh, A; Tang, L; Dao, M; Lu, L und Suresh, S, Fracture toughness and fatigue crack growth characteristics of nanotwinned copper, Acta Materialia, 59 (6), 2437-2446, 2011.

[Son07] Song, YH; Cho, SJ; Jung, CK; Bae, IS; Boo, JH und Kim, S, The Structural and Mechanical Properties of Ti Films Fabricated by Using RF Magnetron Sputtering, Journal of the Korean Physical Society, 51 (3), 1152-1155, 2007.

[Spa77] Spaepen, F, A microscopic mechanism for steady state inhomogeneous flow in metallic glasses, Acta Metallurgica, 25 (4), 407-415, 1977.

[Sto07] Stoyanov, E; Langenhorst, F und Steinle-Neumann, G, The effect of valence state and site geometry on $\mathrm{Ti} L_{3,2}$ and $\mathrm{O} \mathrm{K}$ electron energy-loss spectra of $\mathrm{Ti}_{x} \mathrm{O}_{y}$ phases, American Mineralogist, 92 (4), 577-586, 2007.

[Swa86] Swain, M, Grain-size dependence of toughness and transformability of 2molYTZP ceramics, Journal of Materials Science Letters, 5 (11), 1159-1162, 1986.

[Szl05] Szlufarska, I; Nakano, A und Vashishta, P, A Crossover in the Mechanical Response of Nanocrystalline Ceramics, Science, 309 (5736), 911-914, 2005.

[Tet01] Teter, D; Robertson, I und Birnbaum, H, The effects of hydrogen on the deformation and fracture of $\beta$-titanium, Acta Materialia, 49 (20), 4313-4323, 2001.

[Til05] Tilbrook, M; Reimanis, I; Rozenburg, K und Hoffman, M, Effects of plastic yielding on crack propagation near ductile/brittle interfaces, Acta Materialia, 53 (14), 3935-3949, 2005.

[Tur96] Turner, $\mathrm{M}$ und Evans, A, An experimental study of the mechanisms of crack extension along an oxide/metal interface, Acta materialia, 44 (3), 863-871, 1996.

[Uch04] Uchic, MD; Dimiduk, DM; Florando, JN und Nix, WD, Sample Dimensions Influence Strength and Crystal Plasticity, Science, 305 (5686), 986-989, 2004.

[Uch09] Uchic, MD; Shade, PA und Dimiduk, DM, Plasticity of Micrometer-Scale Single Crystals in Compression, Annual Review of Materials Research, 39 (1), 361-386, 2009.

[Var91] Varias, A; Suo, Z und Shih, C, Ductile failure of a constrained metal foil, Journal of the Mechanics and Physics of Solids, 39 (7), 963-986, 1991.

[Var00] Varias, A und Massih, A, Simulation of hydrogen embrittlement in zirconium alloys under stress and temperature gradients, Journal of Nuclear Materials, 279 (2-3), 273-285, 2000.

[Var02] Varias, A und Massih, A, Hydride-induced embrittlement and fracture in metals - effect of stress and temperature distribution, Journal of the Mechanics and Physics of Solids, 50 (7), 1469-1510, 2002. 
[Ver12] Verma, N; Cadambi, S; Jayaram, V und Biswas, SK, Micromechanisms of damage nucleation during contact deformation of columnar multilayer nitride coatings, Acta Materialia, 60 (6-7), 3063-3073, 2012.

[Voe01] Voevodin, A; Iarve, E; Ragland, W; Zabinski, J und Donaldson, S, Stress analyses and in-situ fracture observation of wear protective multilayer coatings in contact loading, Surface and Coatings Technology, 148 (1), 38-45, 2001.

[Vol02] Volinsky, A; Moody, N und Gerberich, W, Interfacial toughness measurements for thin films on substrates, Acta Materialia, 50 (3), 441-466, 2002.

[Vol06] Volkert, CA und Lilleodden, ET, Size effects in the deformation of sub-micron Au columns, Philosophical Magazine, 86 (33-35), 5567-5579, 2006.

[Vol08] Volkert, CA; Donohue, A und Spaepen, F, Effect of sample size on deformation in amorphous metals, Journal of Applied Physics, 103 (8), 083539, 2008.

[Völ15] Völker, B; Venkatesan, S; Heinz, W; Matoy, K; Roth, R; Batke, JM; Cordill, MJ und Dehm, G, Following crack path selection in multifilm structures with weak and strong interfaces by in situ 4-point-bending, Journal of Materials Research, 30, 1090-1097, 2015.

[Wag12] Wagner, JB; Cavalca, F; Damsgaard, CD; Duchstein, LD und Hansen, TW, Exploring the environmental transmission electron microscope, Micron, 43 (11), 1169-1175, 2012.

[Wan03] Wang, HW; Kang, YL; Zhang, ZF und Qin, QH, Size effect on the fracture toughness of metallic foil, International Journal of Fracture, 123 (3-4), 177$185,2003$.

[Wan07] Wang, Y; Li, J; Hamza, AV und Barbee, TW, Ductile crystalline-amorphous nanolaminates, Proceedings of the National Academy of Sciences, 104 (27), 11155-11160, 2007.

[Was96] Was, G und Foecke, T, Deformation and fracture in microlaminates, Thin Solid Films, 286 (1-2), 1-31, 1996.

[Wei97] Wei, Y und Hutchinson, JW, Steady-state crack growth and work of fracture for solids characterized by strain gradient plasticity, Journal of the Mechanics and Physics of Solids, 45 (8), 1253-1273, 1997.

[Wei05] Weissmüller, J und Markmann, J, Deforming Nanocrystalline Metals: New Insights, New Puzzles, Advanced Engineering Materials, 7 (4), 202-207, 2005.

[Wel61] Wells, A, Unstable Crack Propagation in Metals: Cleavage and Fast Facture, Proceedings of the Crack Propagation Symposium, 1 (84), 1961.

[Wil09] Williams, DB und Carter, CB, Transmission Electron Microscopy, Second Edition, Springer Science, New York, 2009. 
[Wor65] Wortman, JJ und Evans, RA, Young's Modulus, Shear Modulus, and Poisson's Ratio in Silicon and Germanium, Journal of Applied Physics, 36 (1), 153-156, 1965.

[Wu11] Wu, J; Kim, S; Chen, W; Carlson, A; Hwang, KC; Huang, Y und Rogers, JA, Mechanics of reversible adhesion, Soft Matter, 7 (18), 8657-8662, 2011.

[Xu95] Xu, X, Perturbation of the substrate temperature by the impingement of laser ablated particles, Journal of Applied Physics, 77 (12), 6715-6717, 1995.

[Yin12] Yin, D; Peng, X; Qin, Y und Wang, Z, Adhesion, atomic structure, and bonding variation at TiN/VN interface by chemical segregation, Surface and Interface Analysis, 44 (9), 1261-1270, 2012.

[Yok02] Yokoyama, K; Ichikawa, T; Murakami, H; Miyamoto, Y und Asaoka, K, Fracture mechanisms of retrieved titanium screw thread in dental implant, Biomaterials, 23 (12), 2459-2465, 2002.

[You04] Youssef, KM; Scattergood, RO; Linga Murty, K und Koch, CC, Ultratough nanocrystalline copper with a narrow grain size distribution, Applied Physics Letters, 85 (6), 929-931, 2004.

[You05] Youssef, KM; Scattergood, RO; Murty, KL; Horton, JA und Koch, CC, Ultrahigh strength and high ductility of bulk nanocrystalline copper, Applied Physics Letters, 87 (9), 091904, 2005.

[You06] Youssef, K; Scattergood, R; Murty, K und Koch, C, Nanocrystalline Al-Mg alloy with ultrahigh strength and good ductility, Scripta Materialia, 54 (2), 251-256, 2006.

[You14] Youssef, $\mathrm{M}$ und Yildiz, B, Hydrogen defects in tetragonal $\mathrm{ZrO}_{2}$ studied using density functional theory, Phys. Chem. Chem. Phys., 16, 1354-1365, 2014.

[Yu96] Yu, N, Ion-beam-induced epitaxial crystallization of sol-gel zirconia thin films on yttria-stabilized zirconia, Philosophical Magazine Letters, 73 (6), 359-368, 1996.

[Yu12] Yu, Q; Li, S; Minor, AM; Sun, J und Ma, E, High-strength titanium alloy nanopillars with stacking faults and enhanced plastic flow, Applied Physics Letters, 100 (6), 063109, 2012.

[Yu15] Yu, Q; Qi, L; Tsuru, T; Traylor, R; Rugg, D; Morris, JW; Asta, M; Chrzan, DC und Minor, AM, Origin of dramatic oxygen solute strengthening effect in titanium, Science, $\mathbf{3 4 7}$ (6222), 635-639, 2015.

[Zha03] Zhang, W; Smith, J; Wang, XG und Evans, A, Influence of sulfur on the adhesion of the nickel/alumina interface, Physical Review B, 67 (24), 245414, 2003. 
[Zha11] Zhang, J; Zhang, X; Wang, R; Lei, S; Zhang, P; Niu, J; Liu, G; Zhang, G und Sun, J, Length-scale-dependent deformation and fracture behavior of $\mathrm{Cu} / \mathrm{X}$ $(\mathrm{X}=\mathrm{Nb}, \mathrm{Zr})$ multilayers: The constraining effects of the ductile phase on the brittle phase, Acta Materialia, 59 (19), 7368-7379, 2011.

[Zhu04] Zhu, YT und Liao, X, Nanostructured metals: Retaining ductility, Nature Materials, 3 (6), 351-352, 2004.

[Zhu08] Zhu, XF; Li, YP; Zhang, GP; Tan, J und Liu, Y, Understanding nanoscale damage at a crack tip of multilayered metallic composites, Applied Physics Letters, 92 (161905), 161905, 2008. 


\section{Abbildungsverzeichnis}

2.1 Bruchmoden . . . . . . . . . . . . . . . . . . . . . . . 6

2.2 Definitionen von CTOD $\ldots \ldots \ldots \ldots \ldots$

2.3 Bruchmechanismen nc-Materialien. . . . . . . . . . . . . . . . . . . 12

2.4 Anderson Modell . . . . . . . . . . . . . . . . . . . . . 16

2.5 Energiefreisetzungsrate Kupferfolien verschiedener Dicken . . . . . . . . 17

2.6 Verformung einer Metallschicht $\ldots \ldots \ldots \ldots \ldots \ldots$

2.7 Verformungsmechanismus kristallin/amorpher Komposite . . . . . . . . 21

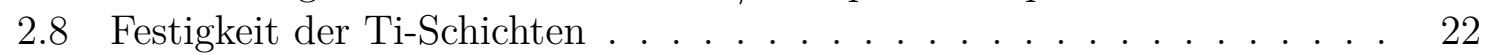

3.1 Schematische Querschnittszeichnung des ETEM . . . . . . . . . . . . 32

3.2 Skizze des STM-TEM-Probenhalters $\ldots \ldots \ldots \ldots \ldots \ldots$

3.3 SEM Aufnahme der keilförmigen Spitze . . . . . . . . . . . . . . . . 36

$3.4 \quad$ Skizzen zur Probenpräparation für Risse entlang der Grenzfläche. . . . . 39

3.5 Skizze zur Probenpräparation im FIB $\ldots \ldots \ldots \ldots \ldots \ldots$

3.6 Skizzen zur Probenpräparation für Risse senkrecht zu den Grenzflächen 41

$3.7 \quad$ Skizzen zum Bruchversuch entlang der Grenzfläche $\ldots \ldots \ldots$. . . . . 41

3.8 Skizzen zum Bruchversuch senkrecht zu den Grenzflächen . . . . . . . . 42

3.9 Skizze zur Bestimmung der Rissspitzenaufweitung . . . . . . . . . . . . 43

3.10 Energiefreisetzungsrate vs. Risslänge schematisch . . . . . . . . . . . 44

3.11 Plastische Zone Ti-Einzelschicht . . . . . . . . . . . . . . . . 45

3.12 Geometrie und Vernetzung COMSOL Simulationen . . . . . . . . 46

4.1 Übersichtsaufnahmen verwendeter Proben . . . . . . . . . . . . . . 50

4.2 Dunkelfeldaufnahmen Probe $\mathrm{Ti50} / \mathrm{ZrO}_{2} 100$ und $\mathrm{Ti10} / \mathrm{ZrO}_{2} 100 \ldots \ldots . \quad 51$

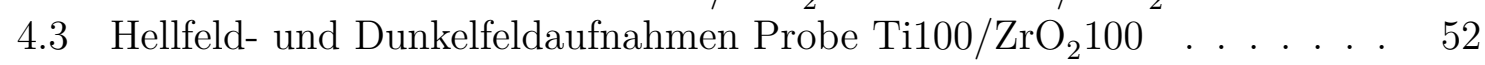

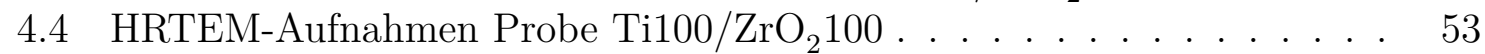


4.5 HRTEM-Aufnahmen Probe Ti50/ZrO 100 und $\mathrm{Ti10} / \mathrm{ZrO}_{2} 100$. . . . . 54

4.6 EELS-Konzentrationsprofile $\ldots \ldots \ldots \ldots \ldots$

4.7 Verformung der Säulen in Simulationen . . . . . . . . . . . . . . . . . 58

$4.8 \quad$ Spannungsverteilung symmetrische Simulation . . . . . . . . . . . . . . . 59

4.9 Spannungsverteilung asymmetrischer Simulationen . . . . . . . . . . . . 60

4.10 Bruchversuch Einzelschicht Titan parallel . . . . . . . . . . . . . . . 63

4.11 Übersicht Riss Einzelschicht Titan parallel . . . . . . . . . . . . . . . . . 64

4.12 Bruchversuch Einzelschicht Titan senkrecht. . . . . . . . . . . . . . . . 65

4.13 Rissübersicht Einzelschicht Titan senkrecht . . . . . . . . . . . . . . . . 66

4.14 Energiefreisetzungsrate vs. Risslänge Ti-Einzelschicht . . . . . . . . . . 67

4.15 Bruchversuch amorphe $\mathrm{ZrO}_{2}$-Einzelschicht . . . . . . . . . . . . . . 68

4.16 Bruchversuch amorphe $\mathrm{ZrO}_{2}$-Einzelschicht . . . . . . . . . . . . . . . . 69

4.17 Bruchversuch kristalline $\mathrm{ZrO}_{2}$-Einzelschicht . . . . . . . . . . . . . . . . 70

4.18 Bruchversuch kristalline $\mathrm{ZrO}_{2}$-Einzelschicht $\ldots \ldots \ldots \ldots$. . . . . . . 71

4.19 Energiefreisetzungsrate vs. Risslänge $\mathrm{ZrO}_{2}$-Einzelschichten . . . . . . . 72

4.20 Bruchversuch Ti100/ $\mathrm{ZrO}_{2} 100$ entlang der Grenzflächen Probe 1 . . . . 74

4.21 Übersicht Riss Ti100/ZrO $\mathrm{Zr}_{2} 100$ erster Schritt Probe 1

4.22 Bruchversuch Ti100/Z $\mathrm{ZrO}_{2} 100$ entlang der Grenzflächen Probe 1 . . . . 76

4.23 Bruchversuch Ti100/Z $\mathrm{ZrO}_{2} 100$ entlang der Grenzflächen Probe 2 . . . . 77

4.24 Übersicht finaler Riss $\mathrm{Ti100} / \mathrm{ZrO}_{2} 100$ Probe 2

4.25 Bruchversuch Ti100/ $/ \mathrm{rO}_{2} 100$ entlang der Grenzflächen Probe 3 . . . . 79

4.26 Bruchversuch Ti100/ $/ \mathrm{ZO}_{2} 100$ entlang der Grenzflächen Probe 4 . . . . 80

4.27 Bruchversuch Ti100/ZrO 2100 entlang der Grenzflächen im SEM Probe 581

4.28 Bruchversuch Ti50/ZrO 2100 entlang der Grenzflächen Probe 5 . . . . . 84

4.29 Bruchversuch Ti50/Z $\mathrm{ZrO}_{2} 100$ entlang der Grenzflächen Probe 5 . . . . . 86

4.30 Bruchversuch Ti10/Z $\mathrm{rO}_{2} 100$ entlang der Grenzflächen Probe 1 . . . . . 88

4.31 Bruchversuch Ti10/ $\mathrm{ZrO}_{2} 100$ entlang der Grenzflächen Probe 1 Riss . . 89

4.32 Bruchversuch Ti10/ZrO 2100 entlang der Grenzflächen Probe 1 EELS . 90

4.33 Bruchversuch Ti10/ $\mathrm{ZrO}_{2} 100$ entlang der Grenzflächen Probe 2 Riss . . 91

4.34 Bruchversuch Ti10/ZrO $\mathrm{rO}_{2} 100$ entlang der Grenzflächen Probe 3 . . . . . 93

4.35 Bruchversuch Ti10/Z $\mathrm{rO}_{2} 100$ entlang der Grenzflächen Probe 3 . . . . . . 93

4.36 Bruchversuch Ti10/ZrO 2100 entlang der Grenzflächen Probe 3 Übersicht

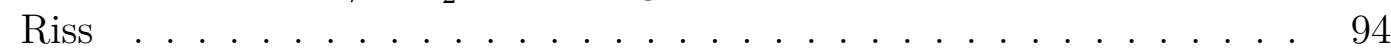

4.37 Größe der plastischen Zone entlang der Grenzflächen . . . . . . . . . . 97

4.38 Energiefreisetzungsrate und Bruchzähigkeit entlang der Grenzflächen . 98

4.39 Risswiderstandskurven Bruchversuche entlang der Grenzflächen . . . . 99

4.40 Bruchversuch Ti100/ZrO 2100 senkrecht zu den Grenzflächen Probe 1 . 101

4.41 Bruchversuch Ti100/ZrO 2100 senkrecht zu den Grenzflächen Probe 1 . 102

4.42 Bruchversuch Ti100/ZrO 2100 senkrecht zu den Grenzflächen Probe 2 . 104

4.43 Bruchversuch Ti100/Z $\mathrm{ZO}_{2} 100$ senkrecht zu den Grenzflächen Probe 3 . 105

4.44 Bruchversuch Ti100/Z $\mathrm{ZrO}_{2} 100$ senkrecht zu den Grenzflächen Probe 3

Übersicht Riss . . . . . . . . . . . . . . . . . 106

4.45 Bruchversuch Ti100/ZrO 100 senkrecht zu den Grenzflächen Probe 4 . 107

4.46 Bruchversuch Ti100/ $\mathrm{ZrO}_{2} 100$ senkrecht zu den Grenzflächen Probe 4 Riss 108

4.47 Bruchversuch Ti50/Z $\mathrm{rO}_{2} 100$ senkrecht zu den Grenzflächen Probe 1 . . 111

4.48 Bruchversuch Ti50/ZrO 2100 senkrecht zu den Grenzflächen Probe 1 Riss 112

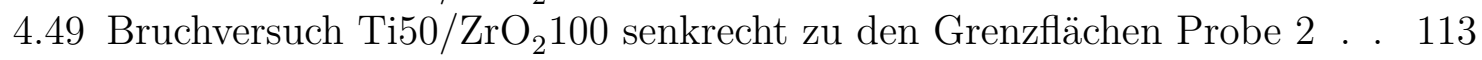


4.50 Bruchversuch Ti50/ZrO 2100 senkrecht zu den Grenzflächen Probe 3 . . 114

4.51 Bruchversuch Ti10/Z $\mathrm{ZrO}_{2} 100$ senkrecht zu den Grenzflächen Probe 1 . . 118

4.52 Bruchversuch $\mathrm{Ti10} / \mathrm{ZrO}_{2} 100$ senkrecht $\mathrm{zu}$ den Grenzflächen Probe 1 Übersicht Riss . . . . . . . . . . . . . . . . . . . . . . 118

4.53 Bruchversuch Ti10/ZrO ${ }_{2} 100$ senkrecht zu den Grenzflächen Probe 2 . . 119

4.54 Bruchversuch Ti10/ZrO 100 senkrecht zu den Grenzflächen Probe 2 Übersicht Riss . . . . . . . . . . . . . . . . . . . . . . . 120

4.55 Bruchversuch Ti10/ZrO 2100 senkrecht zu den Grenzflächen Probe 3 . . 121

4.56 Bruchversuch $\mathrm{Ti10} / \mathrm{ZrO}_{2} 100$ senkrecht $\mathrm{zu}$ den Grenzflächen Probe 3 Übersicht Riss . . . . . . . . . . . . . . . . . . . . . . . . 122

4.57 Bruchversuch Ti10/Z $\mathrm{rO}_{2} 100$ senkrecht zu den Grenzflächen Probe 4 . . 123

4.58 Plastische Zone vs. Ti-Schichtdicke senkrecht . . . . . . . . . . . . . . . 125

4.59 Bruchzähigkeit vs. Ti-Schichtdicke senkrecht . . . . . . . . . . . . . . 126

4.60 Risswiderstandskurven Bruchversuche senkrecht zu den Grenzflächen . 127

4.61 Bruchversuch Ti100/ $\mathrm{ZrO}_{2} 100$ entlang der Grenzflächen WasserstoffAtmosphäre . . . . . . . . . . . . . . . . . . . . . . 129

4.62 Bruchversuch Ti100/Z $\mathrm{rO}_{2} 100$ entlang der Grenzflächen WasserstoffAtmosphäre Riss Lamelle 2 . . . . . . . . . . . . . . . . . . . 130

4.63 Bruchversuch Ti10/ZrO 100 entlang der Grenzflächen WasserstoffAtmosphäre . . . . . . . . . . . . . . . . . . . 130

4.64 Bruchversuch Ti50/Z $\mathrm{rO}_{2} 100$ senkrecht zu den Grenzflächen WasserstoffAtmosphäre . . . . . . . . . . . . . . . . 132

4.65 Bruchversuch Ti100/Z $\mathrm{ZrO}_{2} 100$ entlang der Grenzflächen WasserAtmosphäre . . . . . . . . . . . . . . . . . . 134

4.66 EELS-Analyse in Wasserstoff-Atmosphäre . . . . . . . . . . . . . . . . 135

4.67 EELS-Analyse in Wasser-Atmosphäre . . . . . . . . . . . . . . . . . . . 136

$5.1 \quad$ Skizze zur Bruchgeometrie der Ti-Einzelschichten . . . . . . . . . . . . 140

5.2 Gegenüberstellung a- $\mathrm{ZrO}_{2}$ und $\mathrm{c}-\mathrm{ZrO}_{2} \ldots \ldots \ldots \ldots$. . . . . . . . . 141

$5.3 \quad$ Dundurs Parameter schematisch für Ti/ZrO ${ }_{2}$-System . . . . . . . . . . . 144

5.4 Metall/Keramiksystem . . . . . . . . . . . . . . . . . . . 146

$5.5 \quad$ Modell nach Huang . . . . . . . . . . . . . . . . . . . . . . . . . . . . . . 148

5.6 Versagensmodell nach Zhang . . . . . . . . . . . . . . . . . . . . . . . . . . . 149

5.7 Hell- und Dunkelfeldaufnahme der Rissbrücken in der Ti-Einzelschicht. 149

5.8 Plastische Zone in den Multilagen . . . . . . . . . . . . . . . . 151

5.9 Spannungsverteilung symmetrische Simulation mit Plastizität . . . . . 152

5.10 Ashby-Diagramm . . . . . . . . . . . . . . . . . . . . 154

5.11 Längenskalenabhängige Energiefreisetzungsrate . . . . . . . . . . . . . 156

A.1 SEM-Bruchversuch quantitativ . . . . . . . . . . . . . . . 166

B.1 Zugversuch . . . . . . . . . . . . . . . . . . . . . 168

B.2 Zugversuch während der Verformung . . . . . . . . . . . . . . . . . . . 169

B.3 Lamelle nach Bruch im Zugversuch . . . . . . . . . . . . . . . . . . . . 170

C.1 Kristallisation in $\mathrm{ZrO}_{2}$ im FIB . . . . . . . . . . . . . . . . . . . . 172 


\section{Abkürzungs- und Formelverzeichnis}

BF Hellfeldaufnahme (engl. bright field micrograph)

CCD Ladungsgekoppeltes Bauteil (engl. charge-coupled device)

CTOD Rissspitzenaufweitung (engl. crack tip opening displacement)

DCB Doppelbiegebalken (engl. double cantilever beam)

DF Dunkelfeldaufnahme (engl. dark field micrograph)

EELS Elektronenenergieverlustspektroskopie (engl. electron energy loss spectroscopy)

EDZ ebener Dehnungszustand (engl. plane strain)

EFTEM energiegefilterte Transmissionselektronenmikroskopie (engl. energy filtered transmission electron microscopy)

ESZ ebener Spannungszustand (engl. plane stress)

ETEM atmosphärisches Transmissionselektronenmikroskop (engl. environmental transmission electron microscope)

FEM Finite-Elemente-Methode

FIB Ionenstrahlmikroskop (engl. focused ion beam)

PLD gepulste Laserdeposition (engl. pulsed laser deposition)

HAADF Z-Kontrastaufnahme (engl. high-angle annular dark field)

HRTEM hochaufösende Transmissionselektronenmikroskopie (engl. high resolution transmission electron microscopy)

PDMS Polydimethylsiloxan 
RGA Restgasanalysator (engl. residual gas analyzer)

SEM Rasterelektronenmikroskop (engl. scanning electron microscope)

SIFT skaleninvariante Merkmalstransformation (engl. scale-invariant feature transform)

STEM Rastertransmissionselektronenmikroskop (engl. scanning transmission electron microscope)

STM Rastertunnelmikroskop (engl. scanning tunneling microscope)

STZ Schertransformationszone (engl. shear transformation zone, STZ)

TEM Transmissionselektronenmikroskop (engl. transmission electron microscope)

TMP Turbomolekularpumpe (engl. turbo molecular pump)

XRD Röntgenbeugung (engl. X-ray diffraction)

XRR Röntgenreflektometrie (engl. X-ray reflectometry)

ZLP Null-Verlust-Maximum (engl. zero-loss peak)

a Risslänge

$\beta \quad$ Sammelwinkel des Spektrometers

d Schichtdicke

D Diffusionskoeffizient

$\delta \quad$ Rissspitzenaufweitung (CTOD)

E $\quad$ Elastizitätsmodul

$E_{0} \quad$ Energie der Primärelektronen

$E_{m} \quad$ mittlerer Energieverlust

F relativistischer Korrekturfaktor nach Mal88

$J_{I} \quad$ Energiefreisetzungsrate (engl. strain energy release rate)

$K_{c} \quad$ Bruchzähigkeit oder kritischer Spannungsintensitätsfaktor

$\lambda \quad$ mittlere freie Weglänge der Elektronen

$\mu \quad$ Schermodul

$\nu \quad$ Poissonzahl

$r_{p} \quad$ Radius der plastischen Zone 
$\sigma_{y s} \quad$ Fließspannung

$\sigma \quad$ Unschärfe der Grenzfläche in EELS-Konzentrationsprofilen

$t \quad$ Dicke der Probe in Elektronenausbreitungsrichtung

$t_{D} \quad$ Diffusionszeit

Z Ordnungszahl 


\section{Danksagung}

Ein besonderer Dank gilt Cynthia Volkert für die Betreuung, die Unterstützung und die Diskussionsbereitschaft während der Zeit meiner Doktorarbeit.

Weiterhin möchte ich mich bei Michael Seibt für die Übernahme des Koreferats und die Bereitschaft stets mit mir über meine Ergebnisse und offene Fragestellung zu diskutieren bedanken.

Außerdem danke ich Claus Ropers für die Betreuung während meiner Doktorarbeit.

Robert Maaß und Kodanda Mangipudi danke ich für die Unterstützung und die zahlreichen Diskussionen bei der Entwicklung der Bruchversuche und der Analyse der Ergebnisse. Auch Hans-Ulrich Krebs, Tobias Liese und Inga Knorr danke ich herzlich für die Probenpräparation und die mechanische Charakterisierung der Schichtpakete.

Einen großen Dank möchte ich an das Institut für Materialphysik aussprechen. Viele Mitarbeiter und ehemalige Mitarbeiter haben einen Beitrag zum Gelingen der experimentellen Arbeiten geleistet. Hier möchte ich Matthias Hahn, Vladimir Roddatis, Burkhard Ross, Sönke Schmidt und Torben Erichsen bei der Unterstützung am TEM danken. Weiterhin gilt mein Dank Benedikt Ifland für die Hilfe bei der Aufbringung der Silicium-Schichten und seine Unterstützung während meiner gesamten Doktorarbeit. Außerdem möchte ich mich herzlich bei den Technikern des Instituts bedanken, die viele kleine Probleme schnell und unkompliziert lösen konnten. Ebenfalls danke ich Christian Meckenhäuser für die gute Zusammenarbeit im Rahmen seiner durch mich begleiteten Masterarbeit.

Besonders möchte ich mich bei den aktuellen und ehemaligen Mitgliedern der NanoGruppe für die Zusammenarbeit und Diskussionsbereitschaft bedanken. Meinen Bürokollegen Inga Knorr, Stephanie Mildner, Martin Deutges und Johannes Meier danke ich für eine angenehme Arbeitsatmosphäre.

Meine Familie und meine Freundin Steffi haben einen ganz wesentlichen Teil zum erfolgreichen Gelingen dieser Arbeit beigetragen. Ich danke euch herzlich dafür, dass ihr mir immer liebevoll zur Seite gestanden und mich ununterbrochen unterstützt habt sowie für eurer Interesse an meiner Arbeit. Danke! 
